\title{
NOISE ORIGIN, POWER AND SPECTRA \\ OF DUCTED CENTRIFUGAL FANS
}

by

Roderick Roy Real, B.Sc. (Elect.Eng.) (Saskatchewan)

A thesis presented to the Faculty of Graduate Studies and Research, McGill University, in partial fulfillment of the requirements for the degree of Master of Engineering.

Department of Electrical Engineering, McGill University, Montreal. 


\section{ACKNOWLEDGEMENTS}

Appreciation is expressed by the author to Prof. F.S. Howes of McGill University for guidance, and for handling many administrative details associated with the project.

Financial support of this phase of $f$ an noise research is due entirely to the National Research Council, Ottawa, Canada.

Credit is due Mr. G.E. Chipps, M.E. (McGill, 1956) for constmucting and testing the matched acoustic waveguide; Mr. 4. Bredahl, M.E. (McGill, 1956) for designing, building and testing a suitable windscreen; and the Canadian Sirocco Co. for donating a fan and arranging fabrication of the duct.

The author is indebted to his wife, Irene, for a typing job well done, and for much encouragement. 


\section{ABSTRACT}

Forward and backward curved blade centrifugal fans as nolse sources were subjected to an analysis employing the matched acoustic wavegulde measuring technique.

There are three major findings:

1. The nolse output from homologous serles of fans can be defined by one V - shaped specific nolse power VB. log specific curve, with minimum close to maximum static efficlency. Fan nolse "laws" differ as the slope of the branches of th1s curve.

2. Fan outlet no1se power at maximum fan efficlency approximates $10^{-5}$ times the input fan power.

3. The nolse origin is primarily blade and atr-flow boundary turbulence. The spectrum varies with fan - flow deta1ls. but can be roughly predicted.

The problem of fan design for minimum no1se 18 investigated. 


\section{PREFACE}

"Life is full of sounds, and we want to hear the pleasant and vital ones while shunning the unpleasant and dangerous variety." How increasingly true these words of Lord Rayleigh are in this age .... this age of beautiful "Hi-Fi" reproductions of that universal language of the soul, music .... and also in this age of intense industrialization and urbanization in which silence is fast becoming a most expensive, if not unattainable, luxury.

There is sound which is stimulating, charming, pleasing, uplifting, spirited, beautiful and soothing .... and there is that which is ugly, fatiguing, deafening, depressing and unwanted. This is NOISE; unwanted sound.

The study of noise has evolved into a major branch of acoustics at a time when acoustics itself has scarcely completed its projection from an "art" to a science; and also at a time when civilizations are beginning to realize that there is a by-product of industrial-urban societies which is increasing at a rate much faster than it can be controlled .... noise. It has been observed that there are four major threats to the future of our great cities; overcrowded living and resulting high cost of living, air polution, traffic and NOISE. Unchecked, they may ultimately spelI doom to the metropolis.

Acoustics has modern manifestations in such diverse fields as engineering, architecture, medicine, music, science, psychology, drama and linguistics. The fields of sonics and ultrasonics alike are enjoying an accelerating expansion which may ultimately rival that of a closely allied branch of science and engineering; communications. The focal point of much research in acoustics 
today is noise, its study, reduction and related problems. The research herein reported is such a study. It is but a pinpoint on the map of noise studies to be sure, but like all scientific research it is necessary to exhaust each detail before progressing to the next, lest some salient feature be overlooked.

This thesis is a presentation of the study made of fan noise, a somewhat controversial subject which has been dealt with in a rather empirical manner to date in an effort to provide much needed engineering data. A detailed study is made of a commercial centrifugal ventilating fan possessing forward (Sirocco) and backward (Silentvane) blade curvatures, as outlined in the Abstract.

The problem of noise in ventilating systems is evidenced in the large number of expensive, but noisy, speech-masking installations today. Much of this noise can be attributed to the fan, which may be likened to the generator of an acoustic waveguide network. Fan noise is a nuisance noise .... one which is usually tolerated, often forgotten and considered inconsequential compared to the ocean of noise produced by industry; but one which nonetheless induces fatigue, with all its manifestations and resulting inefficiency and lowered well-being. Most important, it is a needless noise, one which can be largely avoided by careful consideration of a system before installation.

Language and political barriers, plus the rigid limitations of one studying at a Master's level prevent the writer from knowing the vast inheritance in the Scientific Pool of Knowledge. It is believed, however, this research and resulting thesis may be a modest beginning in elevating the study and analysis of fan noise to a more scientific level. 
TABLE OF CONTENTS

Article

Page

Acknowledgements $\ldots \ldots \ldots \ldots \ldots \ldots \ldots$ ii

Abstract $\ldots \ldots \ldots \ldots \ldots \ldots \ldots \ldots$........... ii

Preface...$\ldots \ldots \ldots \ldots \ldots \ldots \ldots$ iv

List of Tables ............... viii

List of Illustrations $\ldots \ldots \ldots \ldots \ldots$ ix

Glossary of Symbols and Terms ...... xii

INTRODUCTION .......... I

1

HISTORY: REVIEW OF THE LITERATURE ... 5

1.1

History of Fan Noise Research .........

6

1.2 Historical Trace of Aerodynamic Noise

and the Nature of Turbulence ...........

Current Status

…

46

2

THEORY ............ 49

2.1

The Matched Acoustic Waveguide ....... 50

$2 \cdot 1 \cdot 1$

2.1 .2

General

Low Frequencies

52

2.1 .3

High Frequencies

2.2

Fan and Turbulence as a Noise Source....

$2 \cdot 2 \cdot 1$

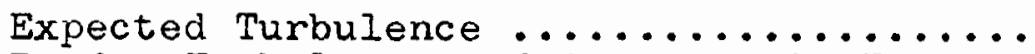

Random Turbulence and Aerodynamic Noise..

2.2.4 The Ducted Centrifugal Fan as a Noise

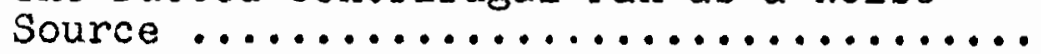

2.3 Hypothetical Ventilating System Noise Example 
4.1.1 Flow - Pressure - Hp. - Speed Data .....

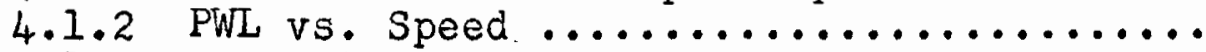

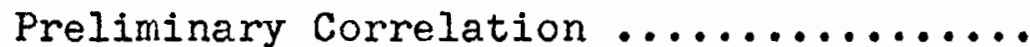

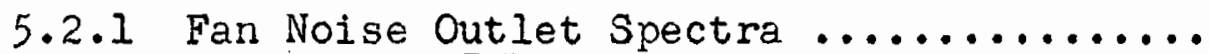

5.3.1 Inlet Noise Spectra and Bandwise ....... Speed Increase

\section{E MOST IMPORTANT RESULTS;}




\section{LIST OF TABLES}

Table

Page

I.I Centrifugal Fan Characteristics ............. 2

1.I Table of Sound Laws for Fans (Ref.54) ......... 14

2.1 Cut - off Frequencies of Some Higher Order Modes. 66

2.2 Noise from Flow over a Flat Plate (Ref.44)...... 81

3.1 SLM Tolerances ........................ 110

3.2 Accuracy of Power to Fan ...................... 117

5.1 Duct PWL and Horn Mouth PWL Compared ......... 193

5.2 Bandwise Increase of Fan Inlet Noise with Speed.. 225

5.3 Simplified Table for Maximum Possible Error in

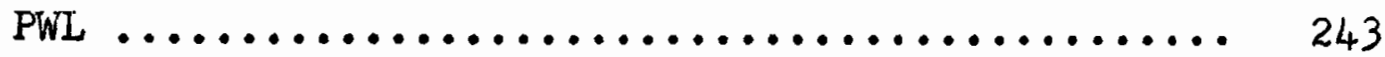




\section{LIST OF ILLUSTRATIONS}

(Note: In Art. 5 where $a, b$ appears after the figure number and two page numbers are given, the first applies to the Sirocco fan, the second to the HS fan)

Figure

Page

1.1

Performance Curves of Four Fans (ref.ll) ...

$1 \cdot 2$

Performance Curves of Three Fans (ref.14,54).

1.3

The Matched Acoustic Waveguide (used in this research)

$1 \cdot 4$

Ducted Axial Fan Noise Test Equipment and

Comparison of Results Between Two Noise

Measuring Methods ...................

Miscellaneous Results from Ref. 4, 18, 27, 38

Calculated Throat Impedance of Modified

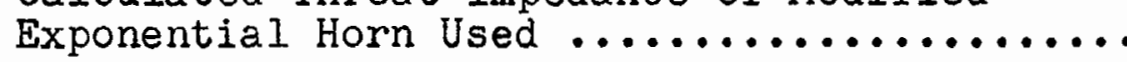

Acoustic Waveguide Behaviour

Various Illustrations Dealing with Aerodynamic Noise Generation

Illustration for Hypothetical Fan Noise

Example

Noise Spectra of Hypothetical Ventilating

System

Spectra at Listener in Hall of Fig. $2.5 \ldots \ldots$

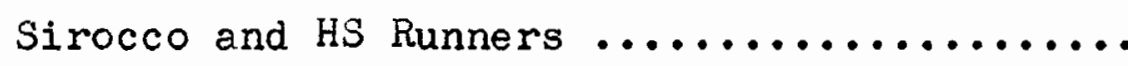

Instruments and Speed Indicator

Performance Curves for the Sirocco and HS

Fan Tested 
5.la, $b \quad$ Noise, Fan, Flow Parameter

131,132

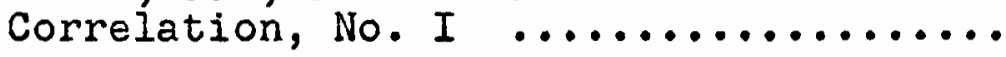

5.2 Minimum Noise and Unit Capacity .......

134

5.3a, b Noise, Fan, Flow Parameter

Correlation, No. II .............. 136,137

5.4a,b Correlation Between Inlet and Outlet

Fan Noise ...................... 141,142

$5.5 a, b \quad$ Noise Power vs. Air Flow

(Constant Speed) ............... 147,148

$5.6 \mathrm{a}, \mathrm{b} \quad$ Noise vs. Flow Velocity

(Constant Pressure) .............. 150,151

5.7a,b Noise vs. Back Pressure

(Constant Air Velocity) ............ 153,154

$5.8 \mathrm{a}, \mathrm{b}$ Noise vs. Speed (Constant System) ..... 156,157

5.9a,b Noise vs. Air Flow and Pressure

(Constant System) ................ 159,160

5.10a,b Noise vs. Hp. to Fan and Specific Speed

(Constant System) ................ 163,164

5.1la,b Specific PWL vs. Air Flow and Pressure

(Constant System) $\ldots \ldots \ldots \ldots \ldots \ldots \ldots \ldots$ 166,167

5.12a,b Specific PWL vs. Speed and Input Power

(Constant System) $\ldots \ldots \ldots \ldots \ldots \ldots \ldots \ldots \ldots$ 168,169

5.13a Sirocco Fan Outlet Noise Spectra ......

171

5.13b HS Fan Outlet and Inlet Noise Spectra ..

172

5.14a,b Outlet and Inlet Fan Noise Octave -

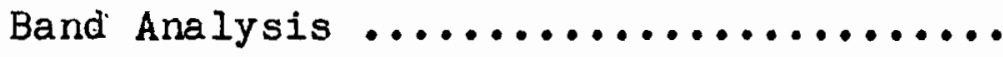

173,174

5.15a Overall Power Level of Sirocco Fan Runner Noise and Bandwise Check of Anechoic Chamber's Approximation to

Free Space

5.15b Overall Power Level of HS Fan Runner

Noise and PWL of HS Fan with

Runner Covered

$5.16 \mathrm{a}, \mathrm{b}$

Fan Runner Spectra

181,182

$5.17 a, b \quad$ Comparison of Sound Level and Air Flow

Contours Beyond Horn Mouth ........... 189,190

$5.18 \mathrm{a}, \mathrm{b} \quad$ Noise Spectra at Horn Mouth ......... 195,196 
5.18c Octave Band Noise Spectra at

Horn Mouth (Sirocco Fan) ............

5.19 Comparison of Flow Spectra with that for Isotropic Turbulence and Mawardi and Dyer's Wind Tunnel Noise .........

5.20 Sirocco Fan Vibration Acceleration and Noise Spectrum .....................

HS Fan Vibration Acceleration and

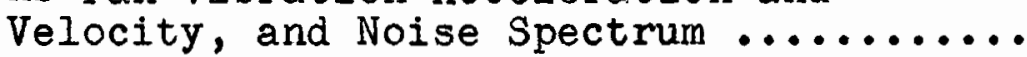

5.23 Overall and Octave-Band Fan Vibration Acceleration vs. Percent Free Flow (Constant Speed) .....................

Overall Vibration Levels; Increase with Speed and Load

$5.25 a, b$ Overall and Octave-Band Fan Vibration Acceleration vs. Fan Speed .......... 212,213

5.26a,b Octave-Band Fan Noise Power vs. Speed ... 215,216

5.27 Octave-Band Fan Vibration plus Blade Turbulence vs... Speed ..................

Octave-Band Runner Noise Power vs. Speed, and Effect of Turbulence on Octave-Band Power Output of Windscreen Covered Microphone

Sirocco Fan Inlet and Vibration Noise Spectra

5.30 Sirocco Fan Vibration Noise and Effect of Dynamic and Aerodynamic Runner Unbalance .........................

Blade Profile PWL and Octave Bands ......

5.33 Spectra from Reversed Fan Runner Rotation 


\section{GLOSSARY OF SYMBOLS \& TERMS}

Other terms appearing in the literature are defined as necessary.

\section{ACOUSTICAL}

$$
\begin{aligned}
a \quad= & \text { Radius of a circular duct or piston. } \\
b_{n} \quad= & \text { nth blade frequency noise component } \\
& =\left(\frac{\text { No. of blades } \times r \cdot p \cdot m \cdot)}{60}\right) n
\end{aligned}
$$

B.W. = Band width. $\begin{aligned} C \quad= & \text { Velocity of sound }=331.4 \sqrt{\frac{T}{273}} \mathrm{~m} / \mathrm{sec} . \\ \mathrm{T}=\text { ambient temperature, } & \mathrm{K} .\end{aligned}$

$f \quad=$ Frequency, c.p.s.

I = Sound intensity, watts/meter ${ }^{2}$

$(I)_{s}=$ Specific sound intensity computed from (PWL), just as I is computed from PWL.

$\mathrm{k}=$ Wave number $=\omega / \mathrm{c}$.

$\mathrm{n} \quad$ = Variation of $\mathrm{k}$, used only if contained in quotations.

NCL $=$ Noise criterean level ${ }^{47}$. It is SIL revised to include annoyance factors.

p = Effective sound pressure at a point, newtons/meter ${ }^{2}$.

$\begin{aligned} \text { PWL }= & \text { Acoustic power level }=10 \log \frac{\mathrm{W}}{W_{\text {ref }}} \mathrm{db} \\ & \mathrm{W} \text { ref. }=10^{-13} \text { watts. }\end{aligned}$

$(P W L)_{s}=P W L-10 \log ($ Qhs 2$) \mathrm{db}$ re $10^{-13}$ watts.

SIL $=$ Speech interference level $=$ average SPL in the 600 to 4800 c.p.s. octave bands.

SLM = Sound level meter.

SWR = Standing wave ratio.

$r \quad=$ Distance of microphone or listener from apparent center of a noise source.

$r_{n}=n^{\text {th }}$ fan rotational component. $=(\mathrm{r} \cdot \mathrm{p} \cdot \mathrm{m} \cdot / 60)$ n c.p.s.

VSWR = Voltage standing wave ratio. 
$\mathrm{Z}_{\wedge} \quad=$ Acoustic impedance, newton-sec $/ \mathrm{m} 5$.

$\mathrm{Z}_{s} \quad=$ Specific acoustic impedance, newton-sec $/ \mathrm{m}^{3}$.

$\mathrm{Z}_{M}=$ Mechanical impedance, newton-sec/m.

$\eta_{A}=$ Efficiency of aerodynamic sound production, $=$ Sound power from fan Power to fan

$\lambda=$ Wavelength.

$\rho_{0}=$ Density of air $=1.29 \quad \frac{273}{\mathrm{~T}} \frac{P_{\mathrm{o}}}{.76} \mathrm{Kg} / \mathrm{m}^{3}$. $\mathrm{T}={ }^{\circ} \mathrm{K}$.

$P_{0}$ - barometric pressure, meters of mercury.

$\omega=2 \pi f$.

MECHANICAL

Air Power = Unless otherwise specified it is $1.575 \times 10^{-4}$ Qhs $x$ 746 watts.

$F_{\text {syst }}=$ Constant system, or system of given frictional resistance to air flow.

Hp in = Horse power to fan.

hs = Static pressure head, inches of water.

ht $=$ Total pressure head $=$ hs + hv .

hv = Velocity pressure head, inches of water.

Mach or $M=$ Mach number of flow - $Y / C$ $(c=$ velocity of sound $)$

n or r.p.m. = Rotational speed, r.p.m.

Ns $=$ Specific speed $=\frac{h \sqrt{Q}}{h_{s}^{.75}}$

$\mathrm{P}_{0} \quad=$ Atmospheric pressure, $\mathrm{cm}$. or $\mathrm{m}$. Mercury

$Q=$ Air flow, c.f.m.

Rey, = Reynold's number of a flow $=\frac{U d}{\nu}$

$d=$ Hydraulic mean depth (duct dia.)

$V=$ Kinematic coefficient of viscosity for air $\simeq$ $1.56 \times 10^{-5} \mathrm{~m}^{2} / \mathrm{sec}$ at $20^{\circ} \mathrm{c}, .76 \mathrm{~m} \mathrm{Hg}$.

Syst. = Refers to a given ventilating system load as seen from the fan discharge. 
$t \quad=$ Temperature, ${ }^{\circ} \mathrm{F}$.

$U=$ Air flow, ft./min. or $\mathrm{ft} . / \mathrm{sec}$.

$U_{f}$ or UFree $=$ Air flow at free fan discharge (when hs $=0$ ) Unit Capacity $=\frac{Q}{\sqrt{h_{s}}}=\frac{A U}{\sqrt{h_{s}}}$ where $A=$ fan outlet area.

$\mathrm{V}=$ Fan blade tip speed, ft./min.

$\eta_{s}=$ Fan static efficiency (based on hs).

$\eta_{T}=$ Total fan efficiency (based on ht).

MISCELLANEOUS

A.S.A. - American Standards Association.

HS = Designation by the American Blower Co. for the "High Efficiency, Streamline" backward curved blade runner (Fig.3.Ib).

N.A.F.M. = National-Association of Fan Manufacturers.

Sirocco $=$ A common North American designation for the forward curved blade fan muner. (Fig.3.la). 


\section{INTRODUCTION}

Air cannot be moved without generating some degree of noise. In a well engineered ventilating system, most of the noise heard at the load (room) is generated by the source (fan). Properly damped and braced ducts (acoustic transmission line or waveguide) should serve to attenuate, rather than generate noise.

Fan noise is broadly due to turbulence at the blades, turbulence of the flow, and vibration, in order of importance at the fan outlet. Considerable research has been devoted to noise from aeroplane propellors and some to the related axial type fan. Less has been done to clarify the relations of noise and noise spectra for the centrifugal fan. This follows since axial type fans are inherently more noisy in operation compared to centrifugal fans and generally the severest noise problems receive first attention. The propellor for example, is, or should be, a slow speed device; but must be operated at high speed in order to derive a practical efficiency from it.

The centrifugal fan is widely employed for ventilating and air conditioning systems, primarily because this type can cover a vast range of pressures and volumes compared to the axial types, which are generally regarded as low pressure fans, although these can be staged in series for higher pressure. Commercial centrifugal fans are roughly categorized as to blade profile; forward curved (Sirocco), radial or straight and backward curved (Silentvane). Each possesses different characteristics as to speed, pressure, capacity relations. The following table will help illustrate this. 
TABLE I CENTRIFUGAL FAN CHARACTERISTICS

(blower application)

\begin{tabular}{|c|c|c|c|}
\hline CHARACTERISTIC & STRAIGHT & FORWARD CURVED & BACKWARD CURVED \\
\hline Speed & Medium & slow & high \\
\hline Usual Pressure Use & $\begin{array}{l}\text { High (tall nar- } \\
\text { row blade }\end{array}$ & medium & medium \\
\hline Number of blades & $10-20$ & $32-66$ & $14-24$ \\
\hline Efficiency & good to high & $\operatorname{good}(60 \%)$ & high $(70 \%)$ \\
\hline Noise & $\begin{array}{l}\text { Not too differe } \\
\text { curved is usual }\end{array}$ & $\begin{array}{l}\text { ent for equal dut } \\
\text { Iy least. }\end{array}$ & y. Backward \\
\hline Blade depth & largest & smallest & medium \\
\hline Hp. chara & $\begin{array}{l}\text { linear rise } \\
\text { with flow }\end{array}$ & $\begin{array}{l}\text { concave upward } \\
\text { with flow }\end{array}$ & convex with flow \\
\hline
\end{tabular}

Fan manufacturers of America have tested the capacity - pressure - speed relationships of fans according to a long established code prepared jointly by the Engineering Committee of the National Association of Fan Manufacturers (NAFM) and the Fan Test Code Committee of the American Society of Heating and Ventilating Engineers (A.S.H.V.E.). Later in the history of fan manufacturing it was decided to supply customers with the overall noise level of each fan type at rated conditions. The contemporary code ( ref. $54, p-304)$ provided for measuring the overall noise at seven stations surrounding the fan while it discharged into a duct fitted with a pitot tube, manometer and throttling arrangement. The fan could be mounted to a foundation or on a wooden beam structure of beams not exceeding $4^{\prime \prime} \times 4^{\prime \prime}$. No vibration isolation was allowed. No standard room or acoustic treatment was provided for except that the background noise was to be $10 \mathrm{db}$ or more below the noise produced by the apparatus. One formula was specified; a correction for variations of fan speed not exceeding $\pm 10 \%$, $\mathrm{db}$ change $=$ $50 \log _{10} \frac{r p m_{2}}{r p m_{1}}$ (the db implying S.P.L. ref. 0.0002 microbars). It was admitted in the code that the sound emitted at the fan dis- 
charge must necessarily be higher than that determined by the code but its exact amount was not measurable because of lack of means for measuring the sound level in an air stream.

To acousticians, the severe limitations of this test code are obvious. Recent research on the measurement of fan noise in the air stream is rapidly changing the picture. A new code should be evolved shortly.

The first signilicant work leading to an improvement of the noise test code on this continent was originated by Beranek, Reynolds and Wilson ${ }^{1}$. Acoustically their apparatus may be classed as a matched, circular acoustic waveguide. It is an instrument; just as electromagnetic waveguide components are used as instruments for measuring the performance of cavity resonators, tubes, etc. The result of subsequent research by Peistrup and wesler2; Beranek, Kemperman and Allen ${ }^{3}$; Goldman and Maling 4 , Van Niekerk ${ }^{5}$, Bredah $1^{6}$ and $G$. Chipps ${ }^{7}$ has resulted in a number of promising emperical formulae concerned with relating the overall noise power output of axial and centrifugal fans to fan $\mathrm{Hp}$, flow, pressure and speed. Some mention of fan noise spectra has also been included. Using these relationships a ventilating engineer can make a crude estimate of the noise level to be expected for a fan over a restricted operating range. As will be shown, much disagreement prevails and the work generally lacks a theoretical background of any sort.

This study proposes what could become a more universal and reliable set of fan noise laws, after more experimentation. By exhaustive noise spectra observations, the origins of centrifugal fan noise can be quite well isolated. Because of the detailed 
nature of this analysis, only two commercial centrifugal fans were covered; the Sirocco (forward curved blade) and Silentvane (backward curved blade). The latter was not studied as thoroughly as the Sirocco, due to the time spent on the former. The random nature of centrifugal fan noise is a stumbling block for accuracy. Wuch more intense, prolonged and detailed studies of fan noise must be done if the problem of fan noise estimation is to be reliably solved and measures taken to minimize it. It needs a scientific foundation. This study provides a modest beginning. It is hoped some of the ideas and results in this thesis help those continuing this line of research, while providing some useable knowledge to the practical fan manufacturer. 


\section{HISTORY: REVIEW OF THE LITERATURE}

As an art, acoustics is ancient, as a science, it was born yesterday. One of the most historically peculiar facts about the field of acoustics was its stagnation, even before the death of its modern father, Rayleigh; then after a time a renaissance which has accelerated the field to fever pitch. The stagnation occurred between 1896 and the late 1920's, at which time industrial concerns began to make use of the subject in telephony and architecture. In contrast to its academic origin, acoustics received its rebirth from industrial origins. Close on the heels of this "re-discovery" of the field was the formation of the Acoustical Society of America in 1928, which served to inject further life blood into the acoustic vein.

As a consequence perhaps of the 20th century trend to high specialization, the view held by Rayleigh that sound and dynamics were two inseparable aspects of the same thing dissolved with the advent of flight. Aeronautics became one field, acoustics another. The development to a high degree of Rayleigh's electric circuit analogies in acoustic systems, plus the vast application of complex electronic instruments in the field, compared to the essentially non electrical instrumentation of aerodynamics, has caused acoustics to become closely associated with electronics and communications in this country.

Fan noise contains a complexity of origins, the major one of which is turbulence at the rotating blades, as is shown to be true in Art. 5. The subject of aerodynamic noise; noise resulting from air turbulence, has only recently received just attention. To understand this type of noise generation requires a re-uniting of acoustics and aerodynamics. For this reason the historically sig- 
nificant past of fan noise research treated in Art.1.I is followed by a review of research on air turbulence and aerodynamic noise, Art. 1.2.

\section{I.I HISTORY OF FAN NOISE RESEARCH}

To avoid needless duplication, the interested reader is also referred to the companion work of G. Chipps 7 which contains a very readable account of fan noise studies and general noise measuring techniques to the present day. A few supplementary notes are hereby incorporated into this brief sketch of fan noise history to follow.

The first laboratory appearance of the fan in acoustics was in 1830. F. Savart $5^{5}$ using fans and toothed wheels placed the minimum audible frequency at 8 C.P.S. and the upper at $24 \mathrm{Kc}$.

There is little account of any work on the problem of fan noise until the acoustics renaissance in the late 1920's. Many books appeared at this time, most of which interpreted and applied Rayleigh's work. The studies which were made, typical of which are ref. 8,9 , concerned themselves generally with methods of minimizing fan and ventilating noise and the standardization of techniques for measuring noise. The work was under severe handicap because:

1. The sound measuring systems of the day were inaccurate and not altogether reliable.

2. A reference level for the noise measured had not been widely decided upon.

3. Little was known of the ear, although loudness contours by Fletcher and Munson came out about this time.

Even today the American Standard Sound Level Meter (A.S.A. spec. $Z 24.3$ - 1944) has rather liberal frequency tolerances for a scientific measuring instrument; and how we hear still remains 
a puzzling problem in modern psychophysics, as no theory of audition proposed has been completely accepted. However, the store of knowledge is building up rapidly.

The reference level of $10-16$ watts / sq. cm. as the average threshold of acute binaural hearing at $1 \mathrm{Kc}$. was published in 1933. This having been proposed, Knudsen', after a review of air conditioning noise, deplored the lack of a sound level meter to simulate the ear.

Marks and Weske" (1934) in considering the design and performance of the axial flow fan came up with the interesting conclusions that:

1. The inherent noise of any fan depends primarily upon the static pressure against which it operates.

$$
d b=C_{4} \log _{. .}\left(\frac{h_{5}}{C_{6}}\right)^{1 / 2}-C_{5} ; h_{5}=C_{6} U_{\text {Flow vel. }}^{2} \text {. }
$$

$h_{s}=$ Pressure; inches of water; the others are constants.

2. Noise and efficiency are not related upon any energy basis because of the exceedingly small amount of energy required to produce noise.

3. Increase in noise generally precedes loss in fan efficiency; hence is a much more critical indicator of proper application than efficiency. Minimum noise corresponds with maximum efficiency.

4. A comparison between axial types and a centrifugal fan shows the noise variations to be much different.

5. In general, a concentric circumferential obstruction of the fan outlet increases the acceleration component of noise, while any unsymmetrical obstruction (such as a motor support) at the inlet of the axial fan may increase the blade frequency components and certain harmonics. The effect with the obstruction at the outlet is similar, but reduced. These effects can be remedied by the use of straightening vanes to minimize the rotational component.

Their findings in graph form have been traced in Fig. 1.1 In connection with point 5, since the airflow at inlet and discharge of a centrifugal fan does not possess a marked rotational component, the effects of such obstruction at inlet 
Fig. 1.1
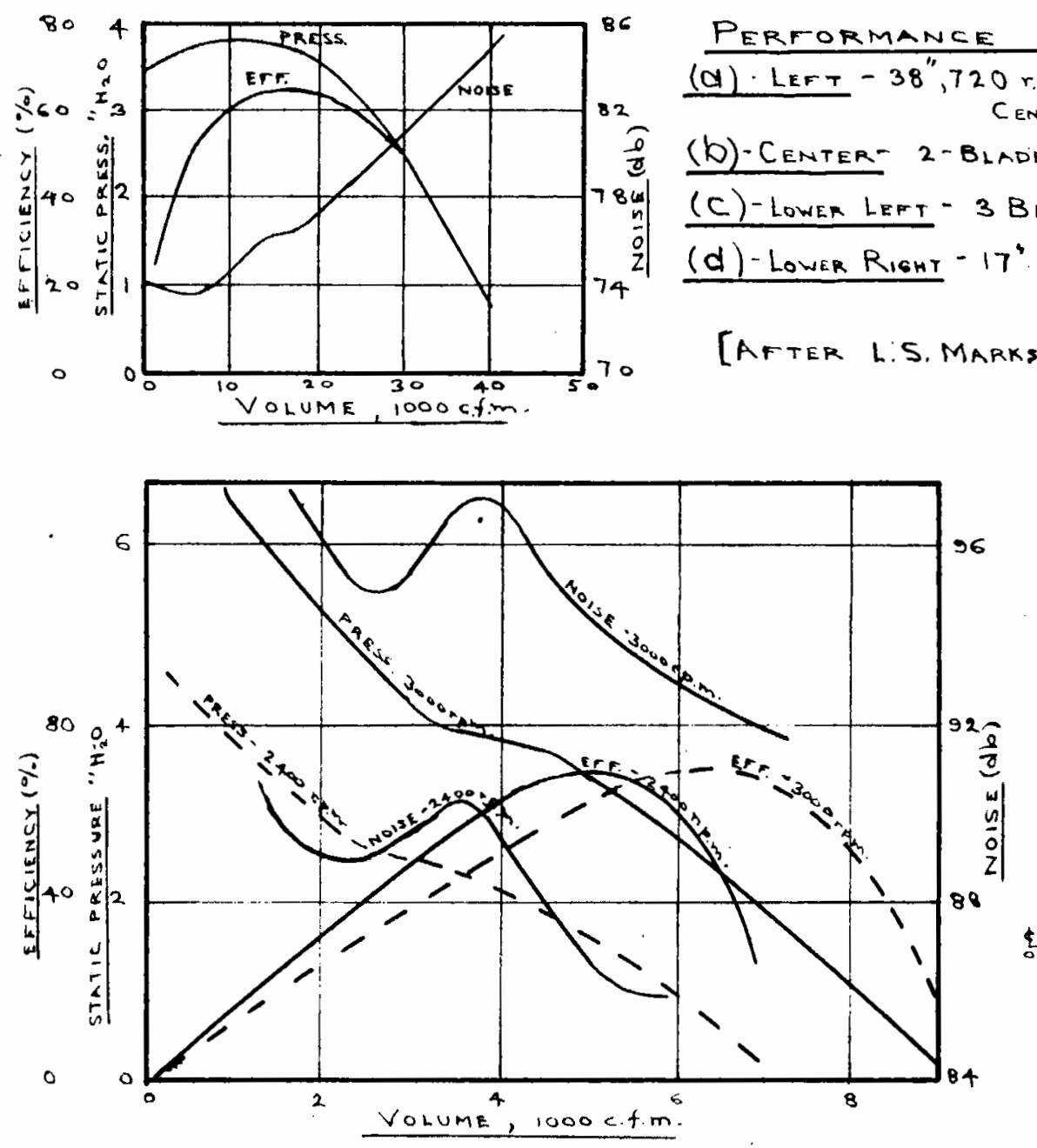

$\frac{\text { Noise Measupimg }}{\text { Stations: }}$

$\frac{\text { Noise Measurimg }}{\text { Stations: }}$

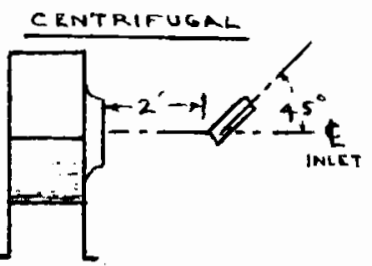

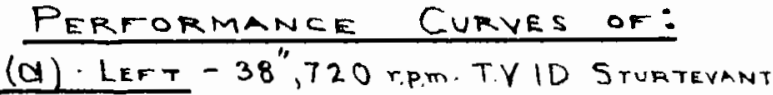

(b)-CENTER- 2-BlADE AXIAL FMN

(C)-Lower LeFt - 3 Blade Aximlfan

(d)-LOWER RIEHT - $17^{\circ}$ LOW PITCH, 1725 r.p.m APhONIC Fan.

[After Lis. Marks \& J.R. Weske]

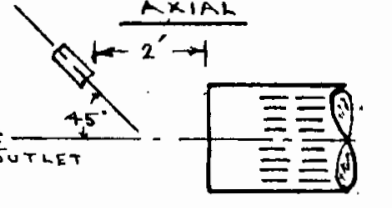

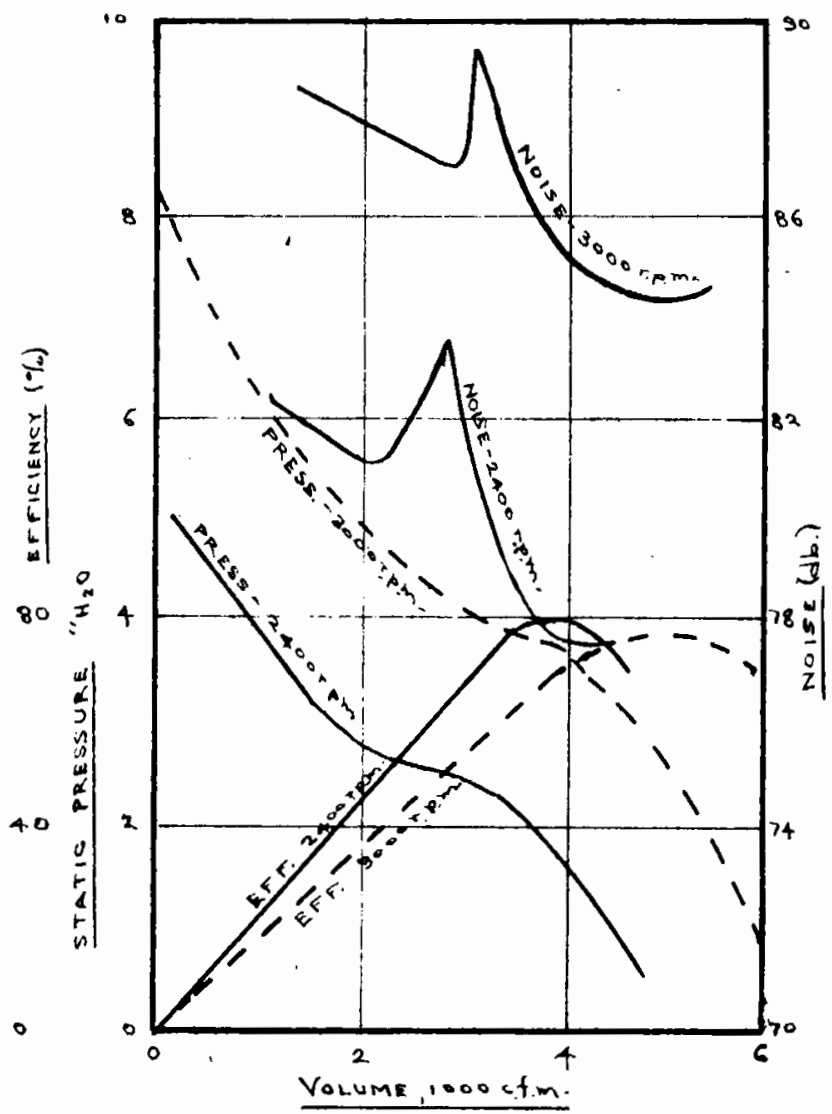


and outlet should have negligible effect, except to change the pressure and flow symmetry. This was confirmed by the writer; no significant change in noise spectra being observed for these conditions.

A concluding note of wisdom by Marks and Weske, "The use of absorbing material should be resorted to after all elements in the fan application have been considered, hence does not represent a problem in fan application".

The noise problem in the application of fans is studied further by McMahan ${ }^{12}$ (1936) who in 1934 also conducted a few simple noise measurements on $12^{\prime \prime}$ and $24^{\prime \prime}$ propeller fans. Although this 1936 research was on axial flow types, he stressed that nearly all the information he collected applied equally well to other fan types. He found that in the normal operating range of axial fans, noise $\propto \sqrt{\max \text {. air acceleration. }}$. He also concluded that the noise is principally dependent upon the static back pressure. The "whine" in high speed propellers and the "flutter" in low speed fans he said were due to the blade frequency and its harmonics. In relating axial fan noise to its operating conditions;

$$
\mathrm{db} \stackrel{\prime}{=} 67 \text { log. } \mathrm{V} .-199.5 .
$$

while for a particular series of similar fans, the noise for a given peripheral velocity varies slightly with fan diameter as:

$$
\begin{aligned}
& d b=67 \log .\left(0.129 d^{1.25} h\right)-199.5 \cdots(1.3) \\
& d b \text { ref. }=0.001 \text { bar (this is balieved to infer microbar) at } 1 k_{c} \text {. } \\
& (1.3) \text { is applicable for } d^{1.25} h>15,000 \\
& d=\text { fan dia. (inches) } \quad h=\text { r.p.m. } \\
& d b=\text { noise in db. } \quad V=\text { peripheral vel. } \mathrm{ft} / \mathrm{min} .
\end{aligned}
$$


McMahan also suggested classes of fan noise which modify the inherent noise of a fan:

(1) Noise increases due to improper flow conditions,

(2) Noise increases due to improper application or aerodynamic design of fan.

(3) Change of inherent noise by other parts of the apparatus,

(4) Amplification by the fan of noise made by other parts of the apparatus

(5) Noise increase due to improper mechanical design or construction.

About this same time, Stowell and Demingl3 (1936) investigated vortex noise from rotating cylindrical rods. One, $1 \frac{1}{2}$ and 2 foot rods, representing propellers, were rotated in a horizontal plane $6^{\prime}$ above ground and studied at a fixed distance from the plane of rotation by a microphone fitted with an extending probe. Their findings are:

(I) Power output in sound $=k l(V)^{5.5} \cdot$. . . $(1.4)$

$k=$ constant

$l=$ length of rod

$v=$ rod tip velocity ( $60_{\text {maters } / \text { sec. max.) }}$

(2) The polar distribution of S.P.L. is similar to a figure 8 with the plane of rotation forming the null axis and the shaft forming the maxima axis. "All rotating systems show this distribution for vortex noise provided that no obstacle of appreciable size is close enough to disturb the diagram."

(3) The spectra all show one maximum which moves out to higher frequencies as the rotational speed increases.

(4) An 18" dia rod when rotating about $2800 \mathrm{rpm}$ emits about 24 microwatts of sound.

(5) From the hub out to 0.81 , the size of the vortices is nearly constant and on the order of a few $\mathrm{mm}$. This size decreases towards the tip.

(6) The spectra were compared with the von Kármán frequency formula

$$
f=\frac{k v}{d} \text { c.p.s. . . . . . }
$$

$V=$ relative velocity of profile 
$d=$ profile dia. or width projected perpendicular to the air stream.

$K=0.194$ for wires, 0.15 for flat plates

This formula says that for nearly all shapes of obstacles, with the exception of aerfoils at normal angles of attack, a rotating profile should generate all frequencies from zero to a maximum determined by (1.5). Compared with the spectra, fair accuracy was attained. Also, use of $\frac{1}{4} "$ instead of $\frac{1}{2} "$ rods showed the maximum significant generated frequency to double. in ref. 13

In this conrection, Strouhal ( 1878 ), who worked with fine wires stretched across the circumference of a drum in such a way that their motion was perpendicular to their length, found the generated frequency was given by

$$
f=\frac{0.185 V}{d} \text { c.p.s. . . (1.6) }
$$

where $V$ and $d$ have the same meaning as in (1.5)

The publication of a Standard for Sound Level Meters, A.S.A. 224.3, 1936, was a most welcome and improving one. The tuning fork, audiometer and acoustimeter now became instruments of old. The new sound level meter, plus a noise measurement specification by A.S.A. at least put sound measurement in the running with measuring instruments in other fields of science and engineering.

Oddly enough, in spite of this development, the status of fan noise from the point of view of research supplying useful noise information to fan manufacturers remained almost static until after World War II. In the meantime an improved sound level meter and specification was issued (A.S.A. 224.3, 1944). True, some propeller noise studies were conducted, and studies on such topics as anechoic chambers, noise measurement techniques and general ventilating system noise were published, but little pointing to a concrete appraisal and research of the fan noise problem could be found in English literature up to 1950. The 
study of turbulent aerodynamic fields was more active during this interval as will be seen in Art. 1.2.

In connection with aerodynamic noise in fans, Bleier ${ }^{4}$ (1948) pointed out that of the four sources of fan noise: vibrational, mechanical, electrical and aerodynamical, the latter was the main problem, and that this was related to blade design. For a given operating condition, the forward and backward curved blades generated comparable noise. Although the forward curved blade is inherently noisy, it need operate at only half the speed of a backward curved blade for a given capacity, resulting in comparable noise generation. This is illustrated in Fig.1.2 (a), (b) in which traces of Bleier's graphical findings are reproduced.

Perhaps the initial impetus to further research on fan noise in North America was provided by the now familiar work of Beranek et al The writer is unable to appraise the status of fan noise study in other parts of the world due to the language barrier, time element and lack of access to many publications. Scientific literature from England indicates many contributions to the understanding of aerodynamic noise (see Art.1.2) but nothing was found on fan noise research in the narrow sense. Batchelor $^{15}$ (1947) who interprets Kolmogoroff's theory of locally isotropic turbulence states that ".... In many respects characteristics of turbulent motion are not much advanced beyond 1938 . Perhaps more work has been done in the U.S.S.R. than in any other country $\ldots . . . "$

What then were fan manufacturers using as fan noise data? Nothing of consequence, until the issuing of the Sound Measurement 


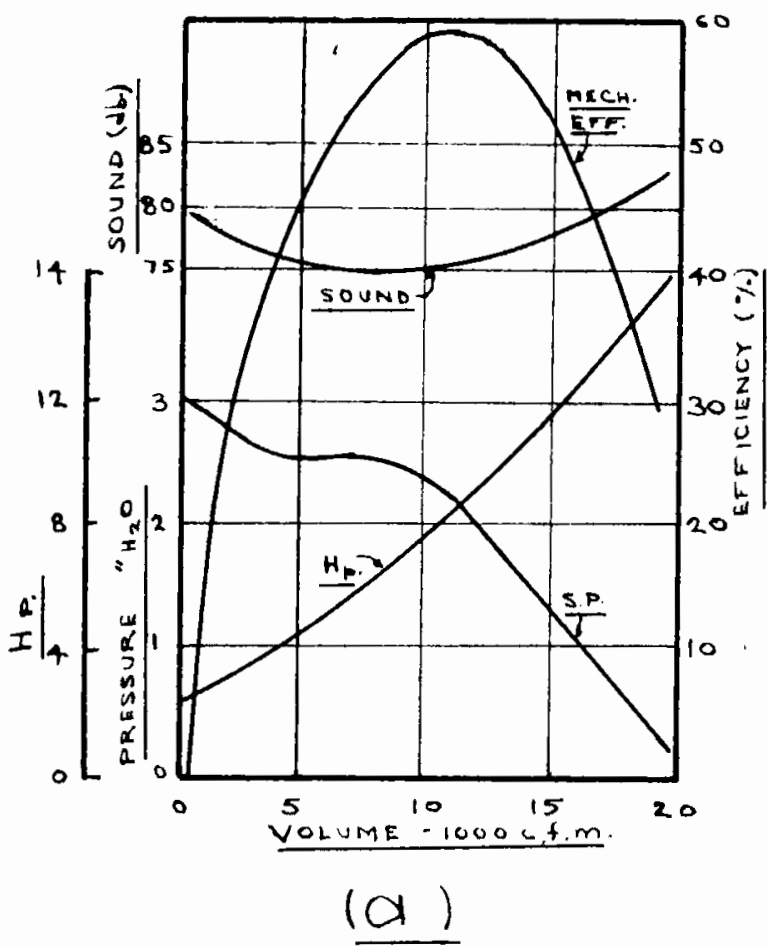

Fig.1.2(a,b) (after F.p. Bleier)

(a) Trpical Forward Blade

Centairugal FAN (27"dia, 570 r.pm)

(b) Typical Backward Blade

Centrifugal Fan (27"dia., 1140 r.p.m.)

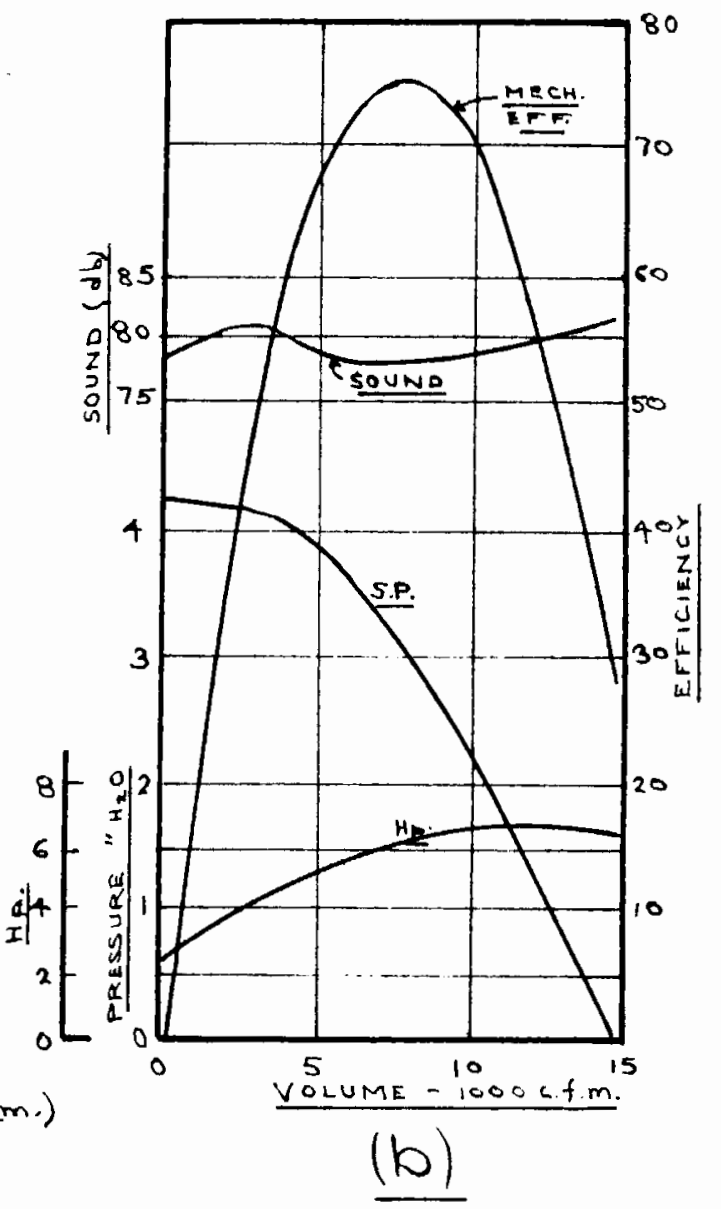

(b)

Fig.1.2(c) (after fan Enginearing)

ILLUSTRATING SPECIFIC SOUND

Performance Curves fora

29 " Axial Flow Fan (with DifPUSER Vanes)

1780 R.PM

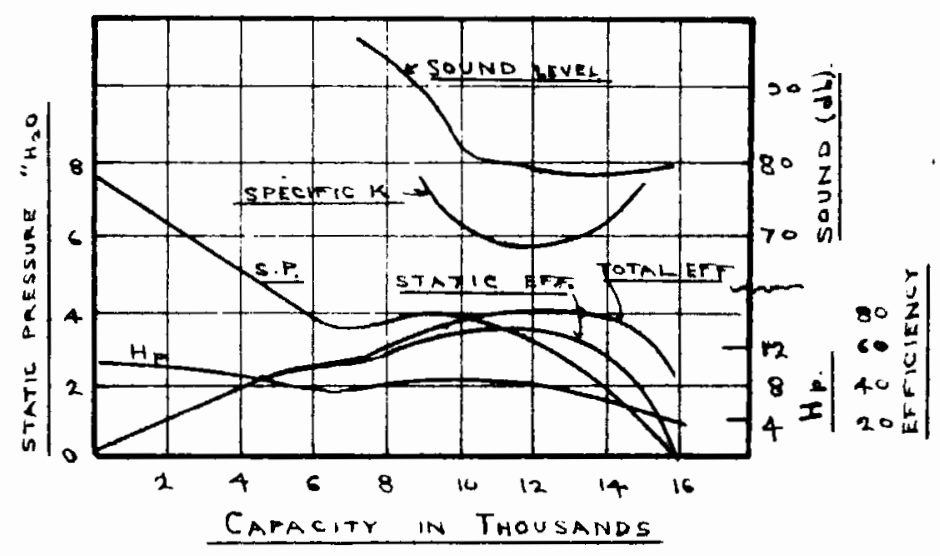


Test Code for Centrifugal and Axial Fans by the N.A.F.M. in 1942. This was briefly reviewed in the introduction. A good practical account on sound appeared in Fan Engineering ${ }^{54}$ (1949). Two major helpful items are included which did not appear generally before:

1. TABLE OF SOUND LAWS FOR FANS (TABLE 2)

(Based on the variation of sound intensity with fan operating conditions)

\begin{tabular}{|c|c|c|c|c|c|c|c|}
\hline 1 & $\Delta I$ & $\alpha$ & atio & $(\operatorname{size})^{7}$ & $x$ & Ratio & $\left(r \cdot p \cdot m_{\bullet}\right)^{5}$ \\
\hline 2 & $"$ & $"$ & $"$ & $(\text { size })^{2}$ & $"$ & $n$ & $(\text { pressure })^{5 / 2}$ \\
\hline 3 & $"$ & $"$ & $n$ & $(1 / \operatorname{size})^{8}$ & $"$ & $"$ & $(\text { capacity })^{5}$ \\
\hline 4 & $"$ & $"$ & $"$ & $(1 / \text { size })^{4 / 3}$ & $"$ & $"$ & $(\mathrm{Hp})^{5 / 3}$ \\
\hline 5 & $"$ & $"$ & $"$ & (capacity) & $"$ & $"$ & $(\text { pressure })^{2}$ \\
\hline 6 & $"$ & $"$ & $"$ & (capacity) & $"$ & $"$ & $\left(\mathrm{r} \cdot \mathrm{p} \cdot \mathrm{m}_{.}\right)^{8 / 3}$ \\
\hline 7 & $"$ & $"$ & $"$ & $(\text { pressure })^{7 / 3}$ & $"$ & $"$ & $\left(1 / r \cdot p \cdot m_{\bullet}\right)^{2}$ \\
\hline 8 & $"$ & $"$ & $"$ & $(H p)^{2}$ & $"$ & $"$ & (I/capacity) \\
\hline 9 & $"$ & $"$ & $"$ & $(\mathrm{Hp})$ & $"$ & $"$ & (pressure) \\
\hline & $"$ & $"$ & $"$ & $(H p)^{7 / s}$ & $"$ & $"$ & $(r . p \cdot m .)^{4 / 5}$ \\
\hline
\end{tabular}

2. THE CONCEPT OF SPECIFIC SOUND

$(d b)_{s}=d b-10 \log$ (c.f.m. $x h_{s}^{2}$ )

$(d b)_{s}=$ specific sound in $d b$.

$\mathrm{db}^{\mathrm{s}}=$ sound in $\mathrm{db}$ according to standard fan test c.f.m.=cubic feet of air per min.

$h_{s}=$ static pressure in inches of water. (Total pressure will serve equally well, but is not used since it is tied up with fan size. However, if free delivery operation is contemplated, $h_{s}=0$ hence (db) must be based upon total or velocity pressure to be determinate. Unless otherwise indicated.specific sound is based on static pressure.)

A few words of discussion about these formulae. It is not known directly just who deserves credit for them; it is not apparent in the seventeen sources listed in the Fan Engineering bibliography at the end of the sound section. Two of the formulae 
have been experimentally verified. Lübcke verified the $7^{\text {th }}$ power law variation with diameter as reported in tests on motor cooling fans. Tests in the Laboratory of the Buffalo Forge Co. over a speed ratio of 2 to $I$ checked the 5 th power ratio of speed change. Two important restrictions apply to the formulae in Table 2:

1."These laws, like the fan laws, apply only at a particular point of rating and cannot be used when moving along the curve from one rating to another.

2."These are not applicable over the entire operating range possible for a fan, but are said to be reasonably close over a very wide range."

These laws have not been verified by experiment (with the two exceptions noted above) but were derived on the basis of the following reasoning:

"The sounds generated by fans follow a fairly well.-de fined system based upon the logarithmic scale. As the fan blades intercept filanents of air there is a pressure built up on one side of the blade and a rarefaction on the opposite side. These pressures are proportional to the pressure developed by the fan since they are notivated by the same means and a change in speed that affects one also affects the other. Since scund intersity varies as the square of the effective sound pressure in the wave it is reasonable that it would also vary as the scuare of the fan pressures developed. For a fan of given pressure the intensity varies as the volune of air flowing throukh the fan inlet or outlet. This is because the intensity varies with the area of flow and area and capacity are proportional for a given velocity pressure. On this basis noise intensity for a given size fan will vary as capacity $X$ (pressure) ${ }^{2}$ or as the 5 th power of the speed." 
The fan laws as in Table 2 follow the same pattern as the ten fan laws (p 242 ref.54) relating size, r.p.m., capacity, press, and $\mathrm{Hp}$.

The specific sound concept could prove to be a powerful tool, as implied in the contents of this thesis. It was derived by analogy with specific speed, a well known concept of fan engineering which enables a homologous series of fansto be defined within narrow limits by a simple curve of static efficiency vs. specific speed. Thether or not the original "unknown" author had sufficient sound data to enable exploitation of this idea, is not known. The extent to which it is used in Fan Engineering is illustrated by the curves of Fig.1.2 (c). Stated is:

1. "It is just as desirnble to relate noise characteristics of a given type and series of proportionately designed. fans as to relate speed - capacity - pressure characteristics. This can be done by plotting a specific sound curve against specific speed or by plotting specific sound against capacity for any given size fan at constant speed."

2."For fan use we will define specific sound as the sound made by a fan of similar type to give one c. $1 . m$. at l" static pressure. Such a theoretical fan would be operating at a speed equal to its specific speed for the rating considered."

3. About fig.1.2 (c): "To fully appreciate the value of specific $K \ldots \ldots$ observe in fig.l.2 (c) that although the actual sound level is relatively flát over a region, it is a mistake to infer this fan may be rated anywhere in that region with equally good results. The specific $K$ curve shows this is not the case ....."

$$
\text { specific } K=d b-10 \log \left(\frac{c \cdot f \cdot m \cdot x h_{s}^{2}}{10,000}\right) \text {. . }
$$

Symbols sane as in eq. (1.7). The use of this gives values nearer to the measured values of sound hence is more easily visualized.

This then has been a summary of ideas the fan engineer has been, and very likely still is, relying upon in North America at least. 
Without omitting much of significance, we come to a group of modern papers ${ }^{1-7}$ which is fast bringing the status of fan noise knowledge to adolescence. They are not elaborated upon here; however their findings are summarized:

Beranek, Reynolds and Wilson ${ }^{1}$ (1953) seem to be the first to publish a paper describing apparatus and procedures for predicting ventilating system noise using a matched acoustic waveguide and microphone windscreen. It is somewhat smaller than the one used by the writer (Fig.1.3), except for the duct diameter which was $211 / 8 "$. They recommend:

(1) A systen constructed closer to N.A.F.M. BuII \#IO - 1950 edition.

(2) Maximum taper of transitions $7^{\circ}$. Conrect fan to circular section of duct equal to fan diameter and 5 to 10 diameters in length.

(3) Make measurements of temperature and barometric pressure at all times.

(4) Undertake further studies on the best position of microphone in the duct.

C.F. Peistrup and Vesler ${ }^{2}$ (1953) using the same apparatus made measurements on three axial and two centrifugal fans. They observed:

1. Overall PijL $=120.4+17.7 \log \left(\frac{H_{P}}{N}\right)+15 \log \left(\frac{N}{6}\right)$ $d b$ re. $10^{-13}$ watts $\cdot(1.10)$ $H p=$ total Hp to fan $\mathbb{N}^{-}=$number of fan biades.

2. A change in back pressure ( $h_{s}$ ) has negligible effect on FwL and spectra.

3. General shape of the exhaust noise spectra curves for any type is essentially independent of fan speed in the range of speeds below rated speed.

4. Most of PI is contained in the lower frequencies.

5. Fundamental blade passage frequency of a fan does not have as great an effect as expected, and is more apparent in the inlet spectra than exhaust. 


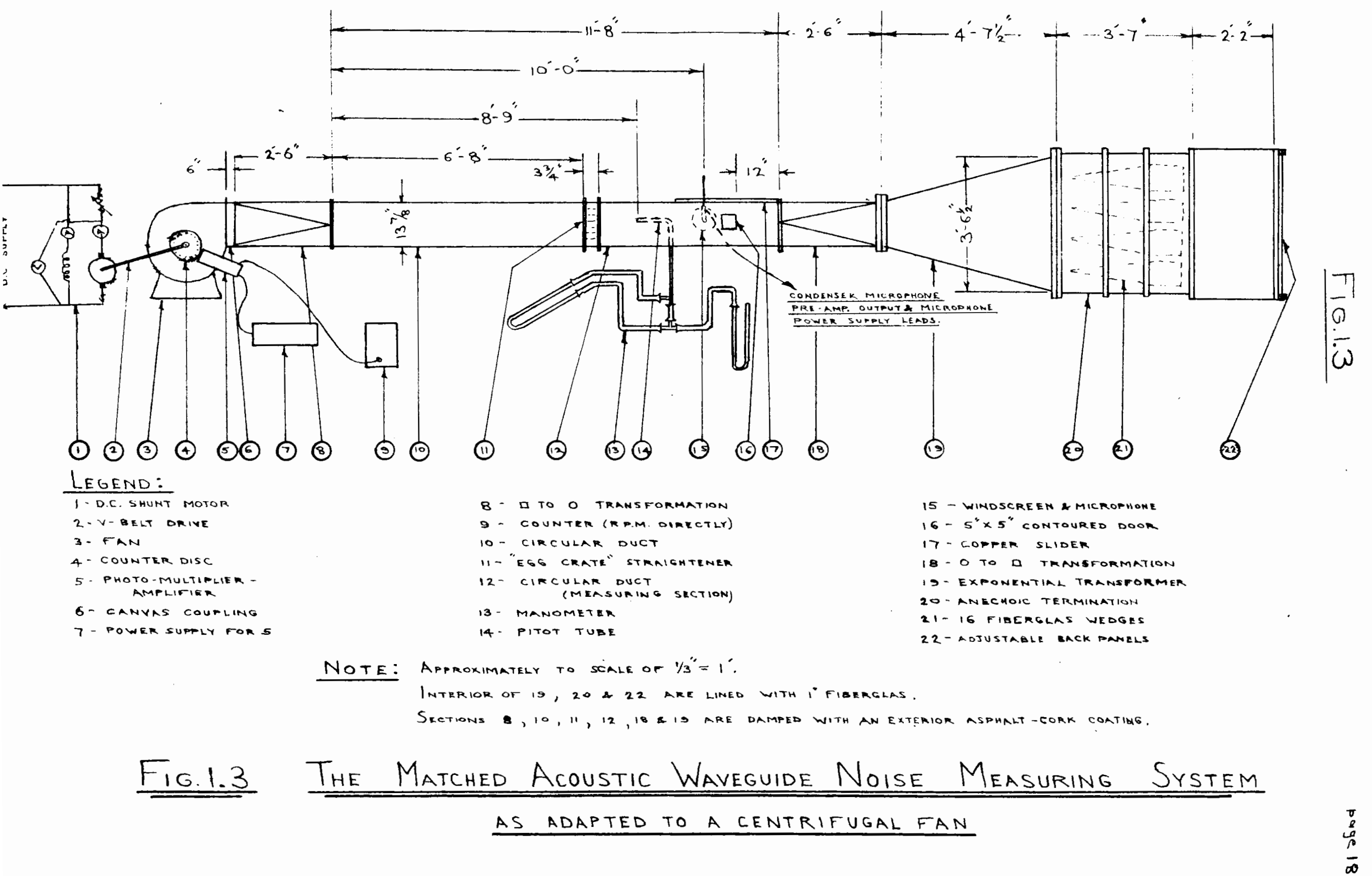


6. In duct systems, the duct dimension in the direction of a bend is a major factor in predicting the attenuation which that duct will provide.

Two years elapsed until another paper on fan noise originated at H.I.T. Beranek, Kamperman and Allen ${ }^{3}$ again looked at the problem of supplying fan manufacturers with suitable PWL vs. fan $\mathrm{Hp}$. data. They made measurements on 14 installed axial and centrifugal fans, making measurements at the fan exhaust or inlet, which ever was accessible, and using the name plate motor Hp. Thereas the noise intensity of a given fan increases with $H p^{2}$, they found that for fans operating near their rated load, noise intensity increases simply linearly with $\mathrm{Hp}$. In properly engineered ventilation systems therefore; overall fan PVL = $100+10$ log $\mathrm{Hp} \ldots \ldots \ldots \ldots$ (I.II) Some of the data of ref. 2 was said to be invalid. Other findings were:

1. Data within $₫ 4 \mathrm{db}$. Scattered overall PWL readings attributed to turbulence in the air stream which produces pressure fluctuations below $75 \mathrm{c} . \mathrm{p} . \mathrm{s}$. Turbulence more prevalent in the exhaust, may be cause of first-band noise.

2. At small fractions of rated speed, Prif does not drop proportionally, perhaps due to the constant operating noises of the mechanical equiprent.

3. For the same rated Ho, two fans may produce significantly different noise. Silentvane is near the lower envelope of the $\pm 4 \mathrm{db}$. variation.

4. At speeds significantly greater than rated values, excessive turbulence sets in which causes a rapid rise in PIIL.

5. PII $\propto 50$ log. r.o.m. relation verified in the region near rated load.

Having provided fan manufacturers with this average emvirical engineering data so that ventilating engineers can determine the anount of noise reduction to be built into a ventilating system, the fan noise studies at M.I.T. ceased. 
Slightly after this last work, Goldman and Jaling studied some small centrifugal fans using a modified, matched, acoustic waveguide system, whereby the air was exhausted through a high acoustic impedance before the microphone, enabling measurement of the fan noise without a windscreen. The study of double inlet Sirocco centrifugal fans rarging from 3" dia., 21" wide to 6" dia., 7" wide netted:

1. Noise intensity $I \propto(\text { air flow })^{5} \ldots \ldots \ldots(1.12)$

2. Noise intensity I $\propto$ (static pressure) $)^{3} \ldots(1.13)$

This inferred that fan noise is separable into

"Flow noise" $\propto(r . p . n .)^{5} \ldots \ldots \ldots \ldots \ldots(1.14)$

$\left.F_{\text {flow }}=\frac{C_{2} Q^{5}}{\alpha^{4} D^{12}} \ldots . . . . . . .15\right)$

and

"Head noise" $\alpha(\text { r.p.m. })^{6} \ldots \ldots \ldots \ldots \ldots \ldots(1.16)$

$P_{\text {head }}=\frac{C_{1} H^{3}}{\alpha}$. . . . . . . . (1.17)

both based on $75-10,000$ c.p.s.

$H=$ static pres. $\mathrm{H}_{2} \mathrm{O} \quad \alpha=$ aspect ratio.

$Q=$ c.f.m. $\quad C_{1}=.9 \times 10^{-4}$ for above units

$D=$ fan dia. $\quad C_{2}=5.2 \times 10^{-10}$ for above units

r.p.m. = fan speed.

Tota.I noise $(75-10,000$ c.p.s. $) \propto(\text { r.p.m. })^{5 \text { to } 6} \ldots($.... (1.18)

(20 - $75 \mathrm{c} \cdot \mathrm{p} . \mathrm{s}$. band was considered unreliable due to

the equiment design).

3. For a fan operating near shut-off "Head noise" dominates. For a fan operating near free flow "rlow noise" dominates. The difference in discharge spectra for these two is shown in fig. 1.5 (c) p 35 .

4. Minimum noise occurs where $P_{\text {head }}=P_{\text {flow }}$. Total noise does not go through a minimurn but approaches the head noise as an asymptote.

5. Minimum noise occurs at maximum fan efficiency.

6. Flow noise is correlated with the fact that vortex noise depends on the 5th or 6th power of the relative velocity between an air stream and an obstacle off which vortices are formed.

7. Noise decreases somewhat with increased aspect ratio. 
8. Least possible noise in a ventilating system is determined only by hs.

9. For minimum noise: choose a fan with large aspect ratio and choose the diameter for maximum efficiency. Speed and $\mathrm{HD}$. are then uniauely determined.

This analysis seems to come the closest so far to explaining centrifugal fan noise.

No papers on the narrow subject of fan noise have made their appearances in internationally known British scientific journals recently although some work may have been done. This writer does not know. One article appeared however in July 1956 from South Africa. Van Niekerk ${ }^{5}$, following the 1953 M.I.T. study by Beranek, Reynolàs and 'ilson', decided to extend research on windscreens and transverse resonance effects in the duct. He employed the novel idea shown in fig.1.4(a), combining a matched acoustic waveguide and reverberant room to make comparisons. His main conclusion regarding fan noise measurement procedures are:

1. Radial variations or pure tones up to $10-12 \mathrm{db}$ at some frequencies above cut - off for the size of duct used.

2. Some Iongitudinal resonance occurred when the system length became multiples of $\frac{1}{t_{r}}$ wave length of the pure tone frequencies under consideration. Unfortunately since these resonant frequencies also coincided with the axial type fan blade frequency and harmonics, the conclusions were not too definite. The fan was run at only one speed.

3. Most interesting is the comparison between noise measured in the duct and in the reverberation chamber. The results (fig. $1.4 \mathrm{~b}$ ) show close agreement above the terminating horn cut-off, and a gradual spread between the two as frequency drops below cut-off. The duct readings are the higher due to more low freauency sound energy remaining in the duct and becoming absorbed by the duct wall as the transmitting efficiency of the horndecreased.

4. It is possible to measure the noise of a fan by means off a reverberation chamber. It may be prohibitively expensive and difficulties may be experienced in coping with the return air flows of large fans with this method. 
Fig.1.4

page 22

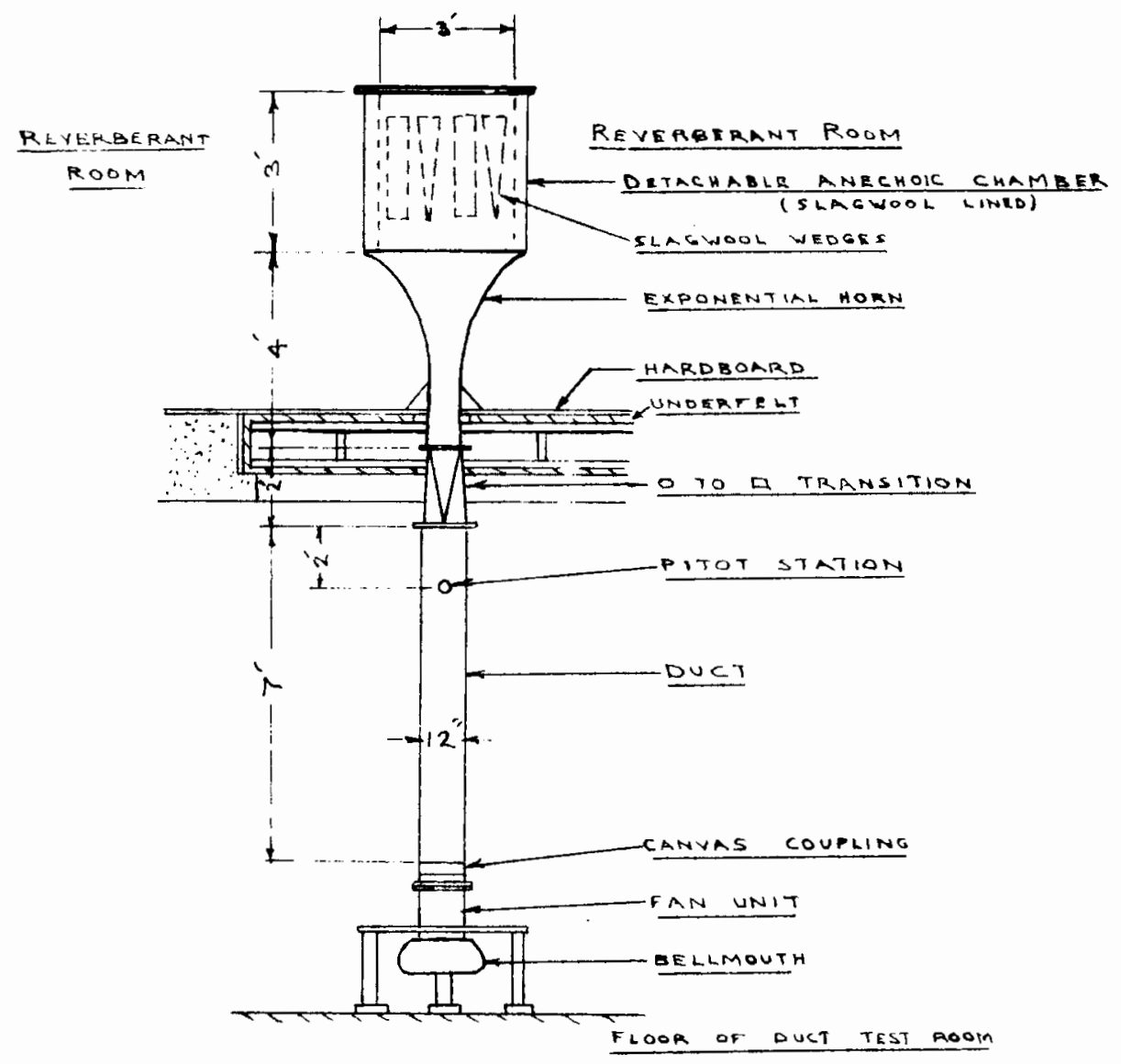

(a) Ducted Axial fan Noise Testing Arrangement Providing for Reverberant Room Comparison (arts Van Niekrake

(b) Mean fan Noise Spectra as Measured in Reverberation $\frac{\text { Chamber \& Duct }}{\text { (artier van nicker) }}$

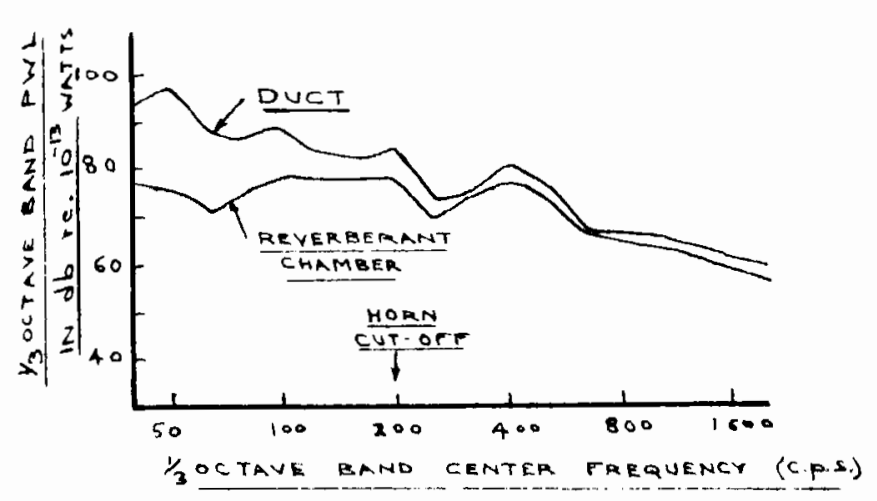


under the direction of Dr. F.S. Howes (this one being the third). One (A. Bredahl) $)^{6}$ deals with windscreens for microphones used to measure noise, when placed in an airstream. "The most effective windscreen from the results is one that gradually reduces the wind velocity and has a framework which will not offer a high impedance to air flow nor distort the desired sound. Light weight nylon tricot and nylon organdy seem satisfactory as a cover for the windscreen frame." "The wind noise appears due to:

1. Turbulence of the air flow

2. Turbulence of the air flow created by the microphone.

3. Vibration of the microphone in an air stream.

4. Bombardment of the microphone by air molecules." A "triple spherical" windscreen built and tested by Hr. Bredahl was adopted for the research in this thesis. (See performance curves in fig $\cdot 3.7 \quad$ ) The other project, by G. Chipps ${ }^{7}$, was the construction and testing of a matched acoustic waveguide to $M \cdot \hat{h} . \vec{H}$. specifications (as far as they went) and the preliminary testing of a Sirocco centrifugal fan (American/Canadiar Sirocco Series 81 It135). The equipment is believed acoustically superior to that of ref. 1. To sumarize his results on the fan (Details of equipment and tests thereor are given in a later section):

1. Determining PiL from a formula based on motor input Hp would yield results which could be in error by as much as 8 to $10 \mathrm{db}$, unless the point of rating on the head flow curve is considered.

2. Near the point of maximum efficiency the overall noise of this fan can be represented empirically by:

$\mathrm{PHL}=100+2010 \mathrm{~g}$. Ho (motor input) db re $10^{-13}$ watts (1.19) This applies for the same point of rating at each $\mathrm{Hp}$.

3. At other than maximum efficiency the noise is higher. The slope of the line representing the variation decreases with increasing back pressure. 
4. A similar condition exists when the results are analysed on a fan $\mathrm{Hp}$ input basis since the motor efficiency varies with operation. For a point of rating near maximum efficiency, (1.19) is now:

$\mathrm{PTI}=106+15.510 \mathrm{Hp}$ (fan) $\mathrm{db}$ re $10^{-13}$ watt $\ldots(1.20)$ The slope of 15.5 agrees favorably with that of 16.6 derived theoretically in Fan Engineering p.312 and verified experimentally in tests conducted to the $N$. A. F.li. test code.

5. The octave band levels do not increase at the same rate with $\mathrm{Hp}$ as the overall levels. The tendency is a. slower decline of P.W.I. with frequency as Hp is increased.

6. At high back pressure, Plit is high, but the octave band levels drop off faster with frequency.

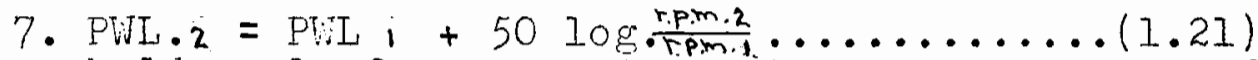
holds only for operating joints near maximum efficiency and near free discharge. It does not hold when moving along the fan performance curve.

8. Overall Prit is a minimum in the region of maximum efficiency.

9. The fan blade frequency does not appear prominently in the noise levels.

10. The octave bands increase with speed with slopes varying from 4.4 . to 6.6 .

11. Noise - level increases with back pressure according to 23.5 loghs except near shut-off where the slope is 15 .

In restrospect, one would tend to agree with Ashley ${ }^{46}$, "We do not yet have a well recognized procedure for the measurement of the PWL of air conditioning equipment, nor a suitable rating code. Very little information is available on the generation of aerodynamic noise in duct systems. Good quantitive data is lacking on the attenuation of duct systems. Data on performance of sound absorbing elements is incomplete. There is no general agreement on standards of desirable and acceptable noise characteristics for different types of room use."

Progress is being made, but much work renains to be done. The state of things may be illustrated by the general acceptance 
of fan noise speed correction in America of (ratio r.p.m. $)^{5}$ and in England ${ }^{55}$ a correction of (ratio r.p.m.) ${ }^{5.5}$, Some progress towards stabalized formulae is believed indicated in this thesis.

\subsection{IISTORICAL TRACE OF AERODYNLIIC NOISE}

AND THE MATURE OF TURBULENCE

.... The roar of the gale .... an observed phenomena since the origin of man has to this day barely been scientifically explained. "The irregular random motion of snall fluid masses to which the name turbulence is given is of such complexity that there can be no hope of a theory which will describe in detail the velocity and pressure fields at every instant." 16

Two major reasons for the lack of great historical development in aerodynamic noise research are:

1. The former tendency to study not only steady state, but impulse and transient wave phenomena, employing Fourier's sinusoidal analysis of amplitude-phase relations.

2. The post Rayleigh divorce of acoustics and aerodynamics. of this Hardy" states: "The statistical approach in terms of energy and intensity relations is the realistic way of studying most noise phenonena ... Much of the noise of modern mechanism is associated with air turbulence, yet very little has been done scientifically to relate the parameters of turbulence and acoustic propagation ... mainly because there are two scientific rather disunited fraternities associated with the dynamics of the atmospheric medium - acoustical and aerodynamical - whose technologies do not even have semantic sympathy."

Aerodynamic noise study began flourishing with Rayleigh.

In his classical, and still useful "Theory of Sound" he investi- 
gates vortex motion, "sensitive" jets, singing flarnes and aeolian tones. "A large and important group of acoustical phenomena have their origin in the instability of certain fluid motions of the kind classified in hydrodynamics as steady ... " A motion, the same at all times, satisfies the dynamical conditions, and is thus in a sense possible; but the smallest departure from the ideal so defined tends spontaneously to increase, and usually with great rapidity according to the law of compound interest. Examples of such instability are afforded by sensitive jets and flames, aeolian tones and by the flute pipes of the organ. These phenomena are still imperfectly understood; but their importance is such as to demand all the consideration we can give them."

As pointed out in Art. 1.1, acoustics growth stagnated from about 1895 to 1928 , and the consideration of aerodynamic noise suffered most of all. The dawn was to break with two major contributions, one on the nature of turbulence itself (Kolmogoroff, 194.1), the other with aerodynamic sound generation (Lighthill, 18,19 1952 and 1954). Prior to these however, research was conducted on mainly turbulence study, typical of which were, "Statistical Heasurements of Turbulence in the Flow of Air Through a Pipe" (Townend, 1934) $)^{20}$, "Imperical Investigation and Analysis of the Velocity Variations in Turbulent Flow" (Simmons and Salter, 1934) ) $^{21}$ and Statistical Theory of Turbulence" (Taylor, 1935) ${ }^{22}$

A host of scientific papers on turbulence have been published in the pasttwenty-five years. It is not intended to give a comprehensive review or bibliography on the subject, but rather to give samples leading up to the important papers on aerodynamic noise. 
in. square pipe. Discharging them together heated small elements of air in the moving air stream in the pipe so that their motions could be photographed by the Schlieren method. Utilizing a microscope, the flow was discovered to be laminar below a Reynolds number (Rey.) of 500. Turbulence studies were performed for $3000<$ Rey $<9300$. The turbulence was a minimum near the axis and rose at an increasing rate toward the wall. Iittle definite phase relation between the maximum values of turbulent components was apparent. Cinematographic records reveal the fluctuating character of the velocity distribution in turbulent flow, which may vary from an almost uniform distribution at one instant to one of very irregular shape. Eddies of fairly regular periodicity sometimes show up, many oi these having wave-lengths $(\lambda)<\frac{1}{2}$ " corresponding to a frequency of about 300 per second.

The affect of a uniform grid or mesh on the turbulence of an airstream was investigated by Simmons and Salter ${ }^{21}$ in a one foot wind tunnel. Using a .0025" dia. hot-wire anemometer he observed velocity changes 6 or 7 times larger in magnitude behind a l" mesh, $\frac{1}{2} "$ thick grid, than those normally present in the wind tunnel. Flow speeds varied from 5 to $55 \mathrm{ft} / \mathrm{sec}$. Values of the average velocity convey some idea of the diffusion behind the grid. Eddy motion occurs from the wake of a strip composing the mesh to the high velocity jet from the neighbouring hole. Mixing was complete in about 10 inches at $5.3 \mathrm{ft} . / \mathrm{sec}$., when turbulence becomes diffuse across the section. This distance increases with speed.

Developing the statistics of turbulence further, Taylor 22 maintained that beyond the "wind shadow" of a grid in an air 
stream, the turbulence therein becomes statistically uniform (isotropic). The scale of the turbulence is anticipated to be some definite fraction of the mesh size of the orid,

$$
\frac{\lambda}{M}=A \sqrt{\frac{\nu}{M u}} \cdot \text {. . . . . . . . }
$$

$\lambda$ may be roughly regarded as the diameter of the smallest eddies responsible for the dissipation of energy.

$M_{1}=$ side of each square of the mesh.

$A=$ absolute constant for all grids of a definite type (e'g. square-mesh, honeycombs). $\nu=$ kinematic viscosity of air. $u^{\prime}=\sqrt{\bar{u}^{2}}=r . m . s$. value of the particle velocity. Rey $=\frac{M u^{\prime}}{\nu}=$ Reynold's number of the turbulence.

Eq. (1.22) is limited:

(I) cannot apply when $\frac{M u^{\prime}}{\nu}$ is small.

(2) cannot apply immediately behind the grid where the wind shadow of the grid is still distinct.

(3) If a very turbulent stream passes through a mesh which is arranged for large scale, (Iarge $M$, thin strips), the original stream turbulence may pass unaffected. As the distance downstream from the grid increases, the large scale turbulence which has passed through it decreases more slowly than that produced by the grid and eventually completely obliterates the effect of the grid.

The results show that although $U^{\prime} / u^{\prime}\left(U^{\prime}=\right.$ d.c. stream velocity) ranges from 4.9 to 110 and $M$ from $0.62^{\prime \prime}$ to $5^{\prime \prime}$, all the $\mathrm{ob}-$ served values of A lie between 1.95 and 2.20, which appears to confirm that this is a universal constant for turbulence produced or controlled by any type of square grid or honeycomb. It also seems that a honeycomb with long cells is more likely to reduce pre-existing turbulence to a definite scale than a grid of say wires or round bars.

Taylor also looks at the distribution of dissipation of energy in a pipe over its cross section and diffusion in a turbulent air stream.

In conclusion, Taylor proclaims that the whole question 
of turbulence clearly needs more experimental work on the lines laid down $\ldots . .$. .

Nore papers appeared between 1936 and 1941 on the general subject of kinenatics of turbulence and the spectra of turbulence, some of which were highly theoretical. simnons and salter ${ }^{23}$ (1938) experimentally investigated the spectrum of turbulence in a 4 foot dia. wind tunnel. They mentioned that there was some evidence that mixing of eddies with high velocity jets behind a mesh across the tunnel continued for a distance of about $15 \mathrm{x}$ the mesh slot dimension, after which the stream is statistically uniform. Taylor ${ }^{24}(1938)$ attempted to compare the results of simmons and salter on the spectrum of turbulence with an analytical anaIysis. A connection between the spectrum of turbulence measured at a fixed point, and the correlation between simultaneous values of velocity measured at two points was sought. A successful comparison was made for isotropic turbulence, but the results were left more or less until theory ripened further.

A review of the statistical theory of turbulence was made by Dryden ${ }^{16}$ (1943) in which turbulent fluctuations, mean motion, vortex trails, pulsations and isotropic turbulence were highlighted. In pointing out the hopelessness of a detailed theory to describe the velocity and pressure fields in turbulence at every instant, he placed existing theories in the empirical or statistical class. The former are focused on the distribution of mean speed and mean pressure, while the latter considers the frequency distribution and mean values of pressure, and the components of the velocity fluctuations.

Randomness is regarded as an essential feature of turbu- 
lence; turbulence is not equivalent to any regular vortex system however complex. Vortex trails are one of a number of distinct velocity fluctuations occurring under certain circumstances. They generally appear when a blunt object (such as a cylinder) is exposed to a fluid stream. The velocity fluctuations in the trail are periodic in themselves and produce no turbulent mixing. The trail pattern quickly transforms into irregular turbulent motion quite unlike any character of the motions in the trail itself.

Space and time averages by hot wire anemometers which average over a cylindrical volume of $0.01 \mathrm{~mm}$ dia, $1 \mathrm{~mm}$ long and over a time interval of $0.5 \times 10^{-3}$ seconds, or in this neighbourhood, are satisfactory. Measuring equipment not approaching these space-time intervals, according to Dryden, gives results which largely reflect the instrument properties, rather than those of turbulence.

Isotropic turbulence (in which changes in magnitude and direction of velocity fluctuation are wholly random) is said to be the simplest type of turbulence for theoretical or experinental investigation. "There is a strong tendency toward isotropy in all turbulent motions. The turbulence at the center of a pipe in which flow is eddying or in the natural wind at a sufficient height above ground is approximately isotropic. A grid of round wires placed in a uniform fluid stream sets up a more or less regular eddy system of non - isotropic character which very quickly transforms into a field of uniformly distributed isotropic turbulence." Turbulent fields of isotropic nature can have a variation in intensity of more than 0.1 to $5 \%$ of the mean speed and a scale from a few $\mathrm{mm}$ to $25 \mathrm{~mm}$.

Dryden goes on to discuss various turbulence generators 
(screens) and concludes that if one studies the turbulent field behind them in relation only to isotropic turbulence, the details of construction and distance upstream of the screen is of no importance.

The exploration of non - isotropic turbulence, Dryden concludes, is still in its earliest stages; handicapped by the absence of reliable experimental data on the twelve functions required to describe the complex state of this turbulence.

Without omitting too much of relevance, we come to the "new and fruitful theory of turbulent motion published in 1941 by A.N. Kolmogoroff". "Strangely enough, this theory did not become widely known outside of the U.S.S. F. until Batchelor ${ }^{15}$ interpreted and extended the paper in 1947. It has not been proved entirely valj.d, but some experimental results have been correlated with this theory. The basic notion is that at high Rey. numbers, all kinds of turbulent motion show a similar structure if attention is confined to the smallest eddies. Motion due to eddies less than a certain limiting size in an arbitrary turbulent field is deterwined unicuely by; (1) the viscosity and (2) the local mean energy dissipation per unit mass of fluid. The physical picture is that the turbulent flow consists of pulsations ranging in scale from a dimension characteristic of the mean flow (which may not be uniform), to a lower limit at wich the motion is entirely laminar. Dach pulsation is an unstable state which passes energy on to ever decreasing pulsations or eddies, urtil in the limit the Rey. number of the smallest pulsations is too small to permit the formation of smaller eddies, at which point viscosity governs the energy balance. The energy for the entire motion lies 
chiefly in the mean flow and the largest eddies, which may be a function of the source producing the flow. A menory - fixing poem of the principle is quoted:

"Big whorls have little whorls, which feed on their velocity; Little whorls have smaller whorls, and so on unto viscosity."

The theory can be applied only when the Rey. number of the flow is sufficiently high for the characteristic size of the smallest eddies to be snall compared to the spacial dimensions of a donain $G$ which contains a "large" number of orders of pulsations. An nth order set of pulsations absorb some energy from the $(n-1)^{\text {th }}$ order and pass it partly to the $(n+1)^{\text {th }}$ order. The energy transfer is affected by: (1) rork done against the Reynolds stresses (corresponding to various rates of momentum flux across surfaces fixed in the fluctuating fluid flow) and (2) The heat energy of the fluid through viscous dissipation. The ratio of energy dissipated through viscosity to energy conveyed to the next highest (smaller) order of pulsations decreases as the Rey. number increases. Consider a domain $G$ where Rey. number is so high that the size of the smallest set of edies < spatial dimensions of $G$. Let the Rey. number fall to a very low value. There may be no eddies small enough to be contained by G. The theory does not hold in this quasi turbulent state. The Rey. numbers of a large percent of engineering flows are well within the range of the theory however. In the upper limiting case of infinite Rey. number, the larger sets of eddies contained by G pass their energy to the next smaller set of eddies, and only the eddies of infinitely small characteristic size will dissipate an appreciable portion of their received energy through viscosity. That briefly is the 
physical picture of this theory. Note that it concerns only the dynamical similarity between turbulence in different states and does not pretend to encompass the passage of turbulence from state to state. As such it provides a powerful tool for the determination of statistical characteristics of space and time derivatives of quantities influerced by the turbulence.

This theory provoked a revision of thought. As Heisenbur $\xi^{25}$ remarked, "In earlier years one thought turbulence was caused by viscosity ..... now it is almost the reverse. A frictionless liquid is a system with an infinite number of degrees of freedom. As soon as one puts energy into this liquid, the energy will be distributed among all degrees of freedom, and what finally results is a certain equilibrium distribution corresponding to the Maxwellian distribution in gases ..... It is the viscosity that reduces the number of degrees of freedom, since it damps very quickly all motion in the very small eddies .... Turbulence is an essentially statistical problem which can be solved without going into any details of the mechanical motion."

The next few years after Batchelor presented Kolmogoroff's "Theory of Locally Isotropic Turbulence" in 1947 brought forth quite a flood of published papers on turbulence.

Air turbulence and transfer processes were studied by Commings, Clapp and Taylor26 (1948). Employing an 11 inch square duct and honeycomb turbulence generator, turbulence level was calculated from a temperature traverse about a heated wire as an axis. The complete instrument, "hot-wire wake angle instrument", gave the turbulence level as the ratio of the cross current fluctuating velocity to the mean velocity at a point. It was 
found that variation in turbulence at high values of turbulence level (25\%) had little influence on heat transfer rates, whereas at low values of turbulence (1\%), variation of the turbulence level had a pronounced effect.

In looking at the spectrum of locally isotropic turbulence, Kovászay ${ }^{27}$ derived the spectrum of fig.1.5a and compares it with the $" 5 / 3$ power law" several previous authors had derived for the medium frequency range by assuming similarity in the turbulent energy transport and neglecting viscosity. A spectrum function $F(n)$ is defined: $\quad \bar{u}^{2}=\int_{0}^{\infty} F(n) d n \cdot \cdot(1.23)$ $u=$ velocity fluctuations in the turbulence $h=$ wave number After Kolmogoroff's theory, the energy passed to higher frequencies becomes less and less as the viscosity dissipates more and more energy into heat. The solution is found as:

$$
\begin{aligned}
& F(n)=F_{0}\left(\frac{n}{h_{0}}\right)^{-5 / 3}\left[1-\left(\frac{n}{h_{0}}\right)^{4 / 3}\right]^{2} \cdot(1.24) \\
& F ., h_{0} \text { are constants } \\
& R_{0}=\frac{1}{\nu}\left(\frac{F_{0}}{h_{0}}\right)^{1 / 2} \begin{array}{l}
\text { is a universal Rey number which can be } \\
\text { obtained from measurement }
\end{array}
\end{aligned}
$$
The energy balance of the spectrum is shown in figd.5(b).

Following this work on "free turbulence", boundary effects were scrutinized. Townsend 28 (1951) looked at both the boundary between a turbulent stream and quiescent air, and the boundary between turbulent flow and a solid (channel flow). The effect of a turbulent stream on quiescent air is to gradually entrain this air. This is due to a group of large - scale slow eddies which distort the boundary so that the surrounding air is brought into closer contact with it. The scale of these is larger than that 


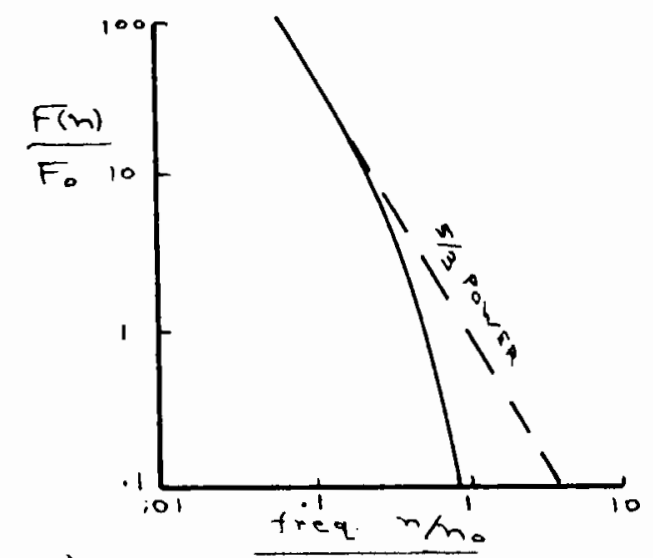

(d) Spectrum of Locally ISOTROPIC TURBulence (b) Energy Balance of $\frac{\text { TURBULENGE SPECTRUM }}{\text { (ATTER KOVMASINAY } 27 \text { ) }}$

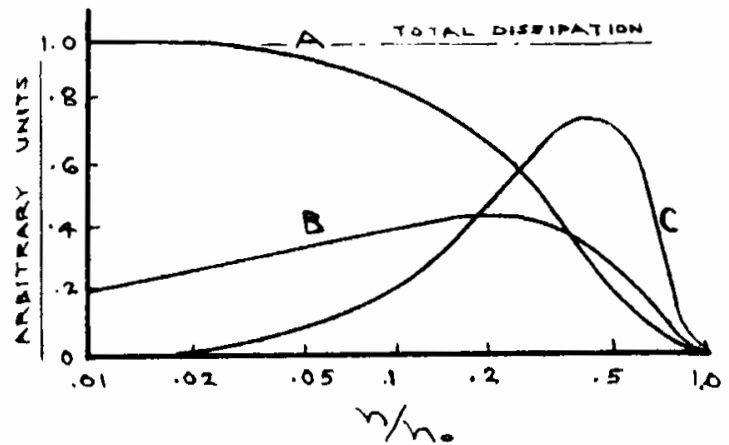

A - S(n) the tUREULENT ENERGY TRANSPORT

B - der THE RATE of VIscous DISsIFATION/FmEQ. dh (WMVE Numaka)

$C-h\left(\frac{d E v}{d h}\right)$ THE MATE of VISCOUS DISSITATION ER TURBULENT "CASCADR - STAGE"

(e.j. PIR OCTAVE)

(C) Noise Spectra of Small Centrifugal FANS

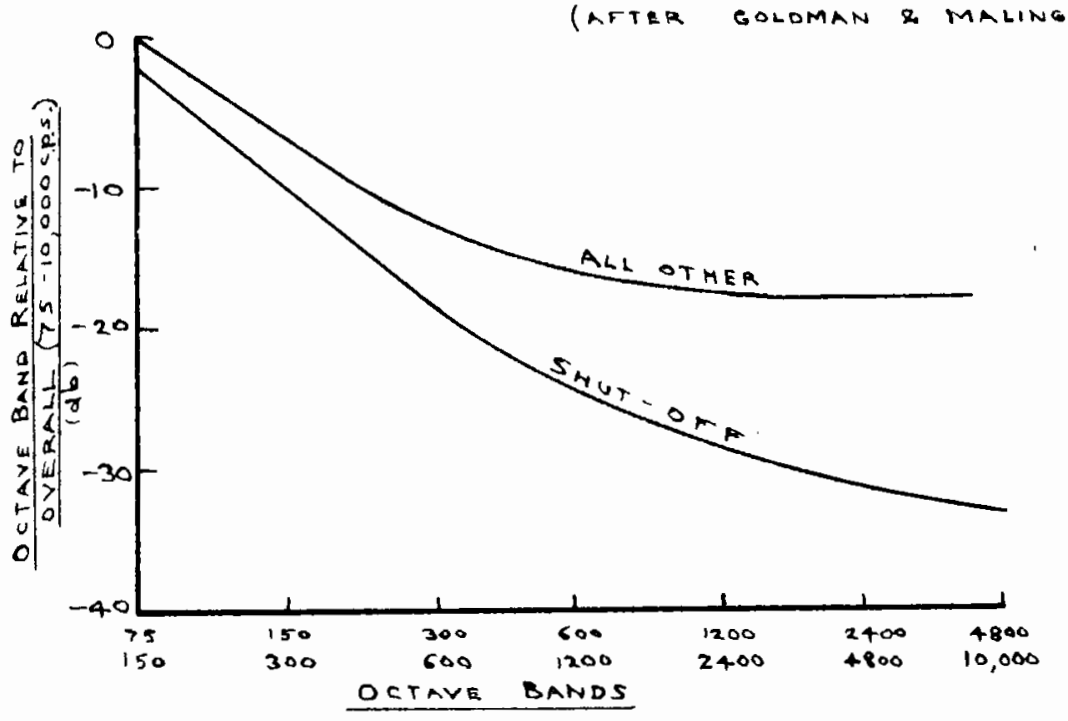

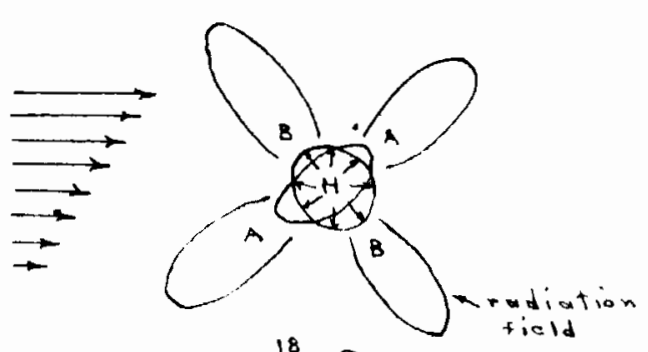

(d) LighthILL'S Quadrupole BASIC LATERML QUAORUPOLE

RESULTING FROM COMQINED EXCESE PRESSURE ANE SHEAR AT P POINT

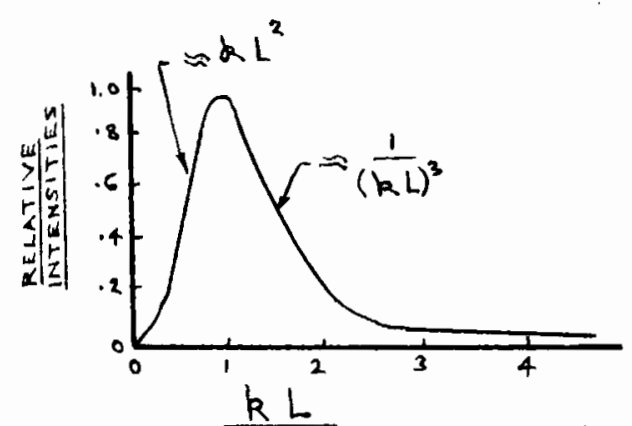

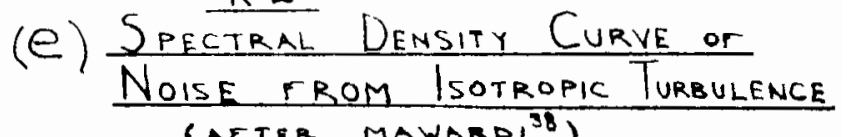


of most of the turbulent motion. Pressure flow in pipes and channels represent the extreme in turbulent shear flow. The properties of the turbulent filuid depend on their position in the flow with respect to the boundary. Towsend concludes that the structure of channel turbulence is not known in comparable detail.

Laufer made measurements on fully turbulent channel flow in a 10 inch pipe at air speeds of 10 and $100 \mathrm{ft} . / \mathrm{sec}$. He suggested that from the standpoint of turbulent structure, the turbulent field may be divided into the following, three regions:

(1) Wall proximity where turbulence production, diffusion and viscous action are all of about equal importance.

(2) The central region of the pipe where energy diffusion plays the predominant role.

(3) An intermediate region between (1) and (2) where the. local rate of change of turbulent energy production dominates the energy received by diffusive action.

There are many physical problems in which pressure fluctuations in turbulent flow play an important part. Batche$\operatorname{lor}^{30}$ (1951) mentions noise as one of them, this being directly related to the fluctuating pressure distribution. In such problems he suggests the usefulness of knowing the statistical properties of the pressure field in a turbulent flow.

Aerodynamic noise received little attention until 1952 when Lighthill $1^{18}$ and Moyal ${ }^{31}$ each presented theories for this method of sound generation. Moyal, by means of an analysis of the kinematics of turbulence of a compressible fluid, coupled the vorticity, dilation, entropy and pressure fields associated with the flow to explain aerodynamic sound. Lighthili's theory however is more advantageous and has been widely acclaimed as being a very important contribution to the theory of aerodynamic sound. His 
theory is based on the equations of motion of a gas. We shall look at it briefiy.

What causes sound? Although the theory of audition is incomplete, it is clearly necessary that suitable pulses of air activate the ear drum. Such pulses can be produced by the vibration of a solid body, as a result of fluctuating masses of air induced by a solid body moving (without vibration) through the air, and finally due to the interaction of fluid in irregular or turbulent motion with fixed surfaces (temporary aerodynamic surfaces). Only the latter is strictly aerodynamic sound, although the second cause is frequently placed in the same category. Each successive cause produces sound less efficiently. When a turbulent fluid moves across the surface of a rigid body, sound is generated. This is also aerodynamic sound and is more efficient than that due to turbulence without solid boundaries, but less than that due to a solid system moving through the air in such manner as to generate sound without vibration (eg. - rotating blades of a fan). All of these sources can be represented by a distribution of dipoles except turbulence without solid boundaries, which is represented by a quadruple distribution. This is Lighthill's contribution.

Consider a fluctuating fluid flow occupying a limited domain of a vast sink of fluid at rest. The momentum or energy within this space can be looked upon as changing at a rate equal to the combined effect of (1) the stresses acting at the boundary and (2) the flow across the boundary of momentum bearing fluid. This is equivalent to an additional stress system referred to as "momentum flux tensor" or "fluctuating Reynold's stresses". A momenturn equation can be written which expresses the fact that mo- 
mentum in any fixed region of space changes at a rate the same as if the gas were quiescent under the combined action of:

(1) the real stresses made up of hydrostatic pressure $p \delta_{i j}$ and viscous stresses, $p_{i j}$.

(2) the fluctuating Reynold's stresses $\rho v_{i} v_{j}$, (i.e. the rate at which momentum in the $x_{i}$ direction crosses unit surface area in the $x_{j}$ direction).

"Since a uniform acoustic medium at rest experiences stresses due to a simple pressure field (the variations are proportional to - density variations with the constant of proportionality being the square of the speed of sound, C2), density fluctuations in the turbulent flow equal those in a uniform acoustic medium subject to an external stress field given by the difference:

$$
T_{i j}=\rho v_{i} v_{j}+p_{i j}-c^{2} \rho \delta_{i j} \cdot \cdot \cdot \cdot \cdot(1.25)
$$

between the effecting stresses on the real flow and the stresses in the uniform acoustic medium at rest". Eq. (1.25) incorporates:

(1) The generation of sound.

(2) Its convection with the flow (in part of the term $\rho v_{i} v_{j}$ ).

(3) Its propagation with variable speed.

(4) Gradual dissipation by conduction.

(5) Gradual dissipation by viscosity (in the viscous contribution to the stress system $\left.P_{i j}\right)$.

In practice, the difference between the exact pressure field and the approximate one $c^{2} \rho \delta_{i j}$, and the contribution of viscous stresses to $T_{i j}$ is unimportant. At low Mach numbers $\left(M=\frac{U}{E}=\frac{\text { flow velocity }}{\text { velocity of sound }}\right), T_{i j}=\rho_{0} v_{i} v_{j}$, and the principal sound generator is fluctuating Reynold's stress corresponding to various rates of momentum flux across surfaces fixed in the fluctuating fluid flow. (The proportional error is on the order of M2). 
The basic equations of the theory of aerodynamic sound production are those representing the propagation of sound in a uniform medium at rest due to externally applied fluctuating stresses:

$$
\begin{aligned}
& \frac{\partial \rho}{\partial t}+\frac{\partial}{\partial x_{i}}\left(\rho v_{i}\right)=0 \cdot \cdot \cdot(1.26) \\
& \frac{\partial}{\partial t}\left(\rho v_{i}\right)+c^{2} \frac{\partial \rho}{\partial x_{i}}=-\frac{\partial T_{i j}}{\partial x_{j}} \cdot \cdot \cdot \cdot \text { (1.27) } \\
& \frac{\partial^{2} \rho}{\partial t^{2}}-c^{2} \nabla^{2} \rho=\frac{\partial^{2} T_{i j}}{\partial x_{i} \partial x_{j}} \text {. . . (1.28) } \\
& \begin{aligned}
T_{i j}= & \text { the instantaneous applied stress at any point } \\
& \text { as discussed above. }
\end{aligned} \\
& \rho=\text { gas density. } \\
& x_{i}, x_{j}=\text { coordinate direction terms. } \\
& \nabla^{2}=\text { Laplacian operator } \\
& P v_{i}, P v_{j}=\begin{array}{l}
\text { momentum across surfaces in } x_{i} \text { and } x_{j} \\
\text { directions respectively. }
\end{array} \\
& C=\text { velocity of sound. }
\end{aligned}
$$

These equations can be used to analyse a turbulent region provided additional mass is not introduced into the region, in which case an additional term must be added.

The novel approach of Lighthill is the evaluation of a general procedure for estimating the intensity of sound produced in terms of flow details, rather than trying to relate frequency in the flow and sound frequency. What is important in acoustics is that it is the intensity at a point, and its frequency spectrum, which is estimated by the ear. At high Rey. numbers when the flow field is fully and randomly turbulent, amplitude and phase have no meaning hence intensity and its spectrum are all that can be given meaning. Using equations (1.25) to (1.28) and these principles, Lighthill evolved a technique for obtaining a general expression for the total 
acoustic output of a turbulent field. The result of the general dimensional analysis was shown to be strongly affected by quadrupole field considerations and the Stokes effect. The amplitude of the quadrupole strength /unit volume is proportional to the square of a typical velocity $U$ in the flow. The amplitude of the radiation field due to a quadrupole (see fig. $1.5 \mathrm{~d}$ ) is proportional to its strength times the square of the frequency (Stokes effect). In many cases a typical frequency is proportional to the flow (Rey. number) so that the amplitude of sound generated by a given fluctuating flow increases roughly as the $4^{\text {th }}$ power of a typical velocity in the flow. In terms of intensity:

$$
I_{\text {sound }} \propto U^{8}(U=\text { typical flow velocity) } \ldots . . .(1.29)
$$

Most of Lighthill's work concerns I and total power output. He described a general method of predicting the spectrum of I from the dimensional analysis, which will not be covered here. He showed that the efficiency of aerodynamic sound production (ratio of the acoustic power output to supply of power) will satisfy roughly:

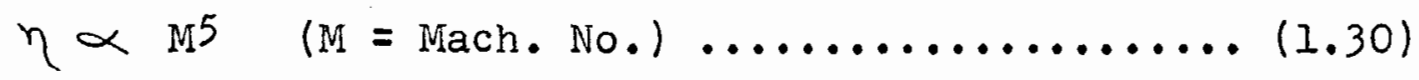

It is evident that acoustic efficiency is very low, even as $M \rightarrow 1$. Gerrard's experiments indicated an order of magnitude of 10-4 for the coefficient $\eta / M 5$. At low Mach numbers, as encountered in ventilating systems, sound from this aerodynamic quadrupole field will nearly always be negligible. As modified by solid boundaries however, it may be significant as mentioned below.

Needless to say, this work of Lighthill revolutionized 
the state of aerodynamic sound knowledge. An increasingly large number of papers followed, many of them devoted to jet noise, due to the pressure of public annoyance being brought to bear on the manufacturers and users of jet planes. One of the first of these was an additional paper by Lighthill ${ }^{19}$ on sound generated aerodynamically. Much of it was devoted to jets. Of direct interest here he states:

(1) In the statistical character of turbulence the values of momentum flux at points with no eddy in common are uncorrelated, while its values at points with many eddies in common are well correlated.

(2) A further consequence of the statistical nature of turbulence is important when the turbulence is superimposed on a mean flow whose Mach number is not negligible.

(3) Although turbulence without a mean flow has an acoustic output, turbulence of a given intensity can generate more sound in the presence of a large mean shear, (solid boundaries become loosely "aerodynamic soundingboards").

A paper received a few months after Lighthill's first on aerodynamic sound was by Proudman, 32 who based his work on a combination of Lighthill's methods, and techniques from the statistical theory of isotropic turbulence. Assuming, as Lighthill did, no back reaction of sound on the turbulence, he stated that in isotropic turbulence only those eddies not dissipating much of their energy through viscosity will make an appreciable contribution to the generation of noise at large Rey. numbers. This he said is independent of the Rey. number. The acoustic power output per mass of the turbulence is

$$
P=\alpha \in M 5
$$

$\alpha=$ universal constant - an order of 10 to 100

$M=$ Mach number $=\mathrm{U} / \mathrm{C}$

$\epsilon=\frac{-3 / 2 \frac{d \bar{u}^{2}}{d t}}{\text { per mass. }}=$ mean rate of dissipation of energy 
Lighthill showed that the fluctuating forces of an air flow on a rigid body may be expected to possess an associated dipole radiation field. Gerrard ${ }^{33}$ found this was so in measuring the sound from circular cylinders in an air stream. For $50<$ Rey. $<105$, essentially pure tone sound was observed to occur. Periodic vortices were shed. Above Rey. of 105 the periodic characteristics of the flow were replaced with the random fluctuations of turbulence, while below Rey. number of 50 no vortices were shed.

It was in 1955 before the influence of solid boundaries on aerodynamic sound was really analysed. As an extension to Lighthill's theory, Curle ${ }^{34}$ incorporated the influence of solid boundaries upon the sound field. Their influence is

(1) Reflection and diffraction of the sound waves at the solid boundaries.

(2) A resultant dipole field at the solid boundaries which are the limits of Lighthill's quadrupole distribution. The quadrupole field still exists throughout the region external to the solid boundaries.

What Curle obtains in his dimensional analysis is a dipole contribution added to Lighthill's quadrupole one. Physically the sound field due to turbulence in the presence of stationary solid boundaries (such as a duct wall) is the sum of that generated by a volume distribution of quadrupoles throughout turbulent space plus that produced by a surface distribution of dipoles over the solid boundaries. The higher the Mach number the more important is the quadrupole contribution, the lower the Mach number the more the important the dipole contribution. The frequencies of sound generated by the quadrupole field are twice those of the dipole field. The frequencies of sound gene- 
rated by the fluctuating forces at the solid boundaries (dipole field) have the same frequency as the fluctuating velocity field, according to Curle. Finally, a dimensional analysis similar to Lighthill's shows that the generated sound intensity at large distances is of the general form

$$
\begin{aligned}
I \propto \rho_{0} U_{0}^{6} a_{0}^{-3} L^{2} x^{-2} \quad \ldots \ldots \ldots \ldots \ldots & (1.32) \\
U_{0} & =\text { typical velocity of the flow } \\
& \vdots=\text { typical length of the solid body } \\
\alpha_{0} & =\text { velocity of sound in fluid at rest } \\
\rho_{0} & =\text { density of fluid at rest }
\end{aligned}
$$

At low Mach numbers this dipole radiation is more efficient than the quadrupole radiation as far as sound generation is concerned.

Phillips ${ }^{35}$ uses Curle's results to consider aerodynamic surface sound as turbulent fluid moves across an infinite flat plate. The acoustic effect is analysed to be a finite net dipole distribution near the leading edge, which becomes vanishingly small with increasing distance down the plate.

Most of the above summary of aerodynamic noise progress stems from British authors. Coming to the experimental side of the picture we have in America Mawardi and Dyer ${ }^{36}$ successfully classifying noises of aerodynamic origin by considering the efficiency of conversion from mechanical to acoustical energy from measurements of wind tunnel, turbojet and air jet noises. After Kovasznay27, for a given set of boundary conditions (free jets, wind tunnels, etc.) a universal spectrum is thought to exist

$$
\bar{u}^{2}=\int_{0}^{\infty} F(n) d n
$$

where $F(n)$, the spectrum of velocity fluctuations" $u$ ", is a function of the wave number . On the basis of dimensional analysis, 
the kinetic energy/unit mass/unit time/ unit frequency $S(h)$ can be determined as

$$
S(n)=k_{1}[F(n)]^{\alpha} n^{\beta}
$$

$\alpha$ and $\beta$ are powers to be evaluated

$k_{1}=$ constant determined by boundary conditions.

$S(n)$ is assumed to depend only on $F$ and $n$. The rate of viscous loss of kinetic energy $L(n)$ is of the form

$$
\begin{aligned}
L(n) & =k_{2} \int_{0}^{n} F(n) n^{2} d n \\
k_{2} & =\text { another constant. }
\end{aligned}
$$

From an energy balance viewpoint

$$
\frac{d S(n)}{d n}=-\frac{d L(n)}{d n}
$$

This defines a differential relation which will yield a unique spectrum $F(h)$ for a given set of boundary conditions for the flow.

Two conclusions valuable in classifying and comparing data collected from different aerodynamic noise generating systems are stated by Mawardi and Dyer:

(1) "The spectrum function of the acoustic source is expected to be a unique function given similar flow conditions.

(2) "The strength of the acoustic source seems to be in some cases proportional to the rate of dissipation of kinetic energy.

"Since it is plausible to assume that dynamically similar systems would have a constant kinetic energy and loss coefficient, the efficiency of conversion of mechanical to acoustic energy should also be a constant for these similar systems". 
Of the three types of aerodynamic noise studied, the relavent one here is wind tunnel noise. Measurements were taken on supersonic tunnels whereas for ventilating systems the Mach number is always below 0.1. They excluded noise extraneous to the wind tunnel proper and directed attention only to noise generated within the tunnel. The measurements were taken by moving the microphone along the tunnel exterior for maximum reading, which was then analysed. The noise within the tunnel was obtained by adding its transmission loss, estimated on the basis of experience on similar walls. Error was claimed to be 5 to $10 \mathrm{db}$ in the low frequency range, becoming less with increasing frequency. The result is the somewhat bell-shaped spectra limits shown in fig.5.19, after normalization with respect to the frequency band of maximum output. It might be argued that in a supersonic flow the shock wave produced much of the noise, since it will vary about a mean position. The spectra shape however belies a narrowly concentrated force pounding on the wind tunnel. Maximum sound was found in the vicinity of the diffusers and shock wave. The Rey. number of the flows investigated ranged from about $9 \times 105$ to $2 \times 107$. By comparison of the spectra with that for the rate of viscous loss of kinetic energy for isotropic turbulence predicted by Kovásznay (see dotted curve fig.5.19), the authors conclude that the character of the noise measured definitely points to an aerodynamic origin.

Later, Medwin, Pietrasanta and Dyer ${ }^{37}$ found that the efficiency of aerodynamic sound production is a function of the supply to exhaust pressures of supersonic wind tunnels. (Tunnel sections of $4^{\prime \prime} \times 8^{\prime \prime}$ to $2^{\prime \prime}$ to $2 \frac{1}{2} "$ ). 
An approximate method was developed for the estimation of the acoustic power frequency spectrum of the sound generated from isotropic turbulence by Mawardi ${ }^{38}$ (1955). The end result is the spectral density curve of Fig.l.5 (e) . L may be taken as the radius of a sphere containing one quadrupole of an assembly extending over the region of isotropic turbulence. The statistical behaviour of the velocities in turbulence are such that the values of velocities at points distant by an amount greater than a typical dimension L, are uncorrelated. The turbulent field then consists of a number of non-overlapping regions of volume $4 / 3 \pi L^{3}$ inside which are localized quadrupole sound sources. In Fig.1.5(e) sound power as a function of kL ( $k$ = wave number of eddies contributing to noise of frequency $\omega=\mathrm{ck}$ ) is completely governed by the behaviour of the average of $v_{0}^{2}$, where $v_{0}$ is the strength of the source due to velocity fluctuations inside $\mathrm{L}$.

This pretty well completes the thumbnail sketch of historical developments of aerodynamic sound research and the associated turbulence studies. Much experimental and theoretical work remains of course. An idea of present studies is afforded by Kraichnan ${ }^{33,40,44}$ who recently treated the pressure field within homogeneous anistropic turbulence and pressure fluctuations in turbulent flow over a flat plate.

\subsection{CURRENT STATUS}

Empirically, fan noise measurement has progressed from a few measurements, with rather poor instruments and arbitrary reference levels, to quite elaborate measuring techniques, with 
standardized references and well developed instruments. Scientifically the subject is still in a low stage of evolution.

An acceptable theory of aerodynamic sound was just recently conceived. Theoretical investigations are rapidly expanding the field and drawing the intimately related branches of acoustics and aerodynamics together. Experimental work is lagging, but along with the jet noise problem, it is quickening pace.

Four methods of studying fan noise are proposed:

(1) Duct test: moderately inexpensive, good results except for low frequencies at which the system resonates. It is also the only system which actually measures noise propagated down a ventilating system.

(2) Free field test: excellent for measuring overall fan P.W.L. with no regard for air flow, provided true free field environment can be obtained. This usually means an anechoic chamber; bukly, stationary and expensive. Outdoor measurements can be taken also if the reflective properties of the ground are known.

(3) Reverberant room test; same category as (2) except not as reliable for measuring spectra.

(4) Duct opening test: similar to (1) except the air flow is exhausted prior to reaching the microphone. It is more expensive and unless very well designed, less reliable for spectra measurements than (I).

The duct test is the category the matched acoustic

waveguide used herein falls.

Fan noise measurement and research is presently at

the following state:

(1) Little theoretical investigation has been performed on the fan as a noise source.

(2) Only the duct test has been investigated fairly well. No reliable comparison has been made between the above methods. 
(3) Emperical formulae only for fan P.W. L. have been developed. They are linked with fan operating parameters. Data conflicts.

(4) No investigation of the contributing sources of fan noise has been reasorably done.

(5) No evaluation of aerodynamic noise in a ventilating system has been made.

(6) The fan engineer has been faced with relatively incomplete and conflicting or unproved data. Little emphasis has been placed on spectra.

(7) No fan noise P.W.L. formulae are applicable beyond an operating range near maximum efficiency. A problem where the fan operates considerably off maximum efficiency may arise when high flow at low head is desired, or a fan is operated with varying load.

Investigations herein contribute something to (3), (4),

(5), (6) and (7). At the outset it must be emphasized that this work is narrowly restricted to one size of centrifugal fan having two types of runners. The detailed information gleaned therefrom has been applied as broadly as possible in the hope it will be of use to those continuing fan noise research, and to fan engineers for eventual application. This is the second fan noise research project to be conducted at McGill University under the direction of Professor F. S. Howes. Dr. Howes has maintained some contact with the M.I.T. noise research group, the National Association of Fan Manufacturers and the American Society of Heating and Ventilating Engineers on the subject of fan noise. 


\section{THEORY}

Throughout the ages, science has evolved in two ways. Either some phenomena was observed, then theories developed to describe the observations; or theories were propounded, and thereafter proved by experinent. The science of acoustics has been particularly vitalized by the first form, since the final recipient of all sound is the ear and the human brain. As Rayleigh philosophied, "Without ears we should hardly care much more about acoustics than without eyes we should care about light." Aerodynamic noise, hence fan noise, is very much a subject thirsting for further theory. Lack of knowledge prevents the writer from contributing anything outside of experinental evidence in this thesis. However, some theorization must precede the display of results, if only to gain perspective of the measured data, its accuracy and importance. The cominercial ventilating fan is here treated as a complex acoustic forcing function which

yet defies mathematical description. The purpose of this research is measurement, analysis and organization of the acoustic output from such a source.

The fundamentals of acoustics and the aerodynamics associated with fans are left to texts on the subject, a few of which are to be found in the bibliography. As far as acoustics is concerned, it is ultimately important to obtain the overall power and its spectrum emanating from the fan, and the noise intensity and its spectrum at the ear of the listener. The transmitting medium may consist of anything ranging from free space, to an enormously complex acoustical network, such as a large air conditioning system. This research and thesis concerns itself with one thing, the overall power output of the fan and its 
spectrum.

Three things merit mention in this section which do not appear in published literature on fan noise, but which could well be hidden in scattered theses on the subject:

1. Evaluation of the matched acoustic waveguide as a means of determining accurately the acoustic output and spectrum of a source.

2. A word on the generation of noise by rotating systerns and air turbulence.

3. A hypothetical example of a noise transmission system from fan to ear. To these we now turn in order.

\section{I THE MATCHED ACOUSTIC WAVEGUIDE (ref. Fig. 1.3)}

2.1 .1

This is one of three general techniques employed to measure the acoustic output of a device, the others being free space and reverberant space techniques. One modification of the acoustic waveguide to measure sound in an air stream is that of Goldman and Maling 4, who withdrew the air through a high acoustic impedance prior to reaching the microphone. The problem of attaining the necessary high shunt impedance over the audio range is a stuay drawing no further mention here.

The matched acoustic waveguide aims in its perfected state at directional "free space". We note at this point that the electrical analogy of duct characteristic impedance is $P_{\circ} C /$ area, whereas acoustically it is simply $\rho_{0} C$ for the plane 
wave. With this in mind the system of Fig. 1.3 becomes a bounded extension of the fan outlet long enough to stabilize air turbulence and allow measurement of the noise, plus a transformer matching the analogous duct impedance $P_{0} c / A_{\text {duct }}$ to $\rho_{0} c$, the characteristic impedance of the "free space" termination. The acoustic output power is obtained from one reading of sound pressure in the duct section, just as it can be sampled at one point on a spherical surface in free space (more if source is directional). Why bother with such a system at all, if free space will suffice?

1. "Tree space" with low ambient noise level is virtually unattainable in urban centers.

2. A good anechoic room is costly and not portable. The same applias to a reverberant chamber.

3. Sampling the noise at the fan outlet or inlet is influenced by localized effects such as disregard for impedance matching, mutual interference of inlet and outlet noise, effect of room reverberation on the measurements, unknown and unstable seale of air turbulence at these positions and lack of practical back pressure control.

4. Most fans are ducted, and such a measuring system most closely approximates the installed fan in practice.

5. By ideans of duct length and a grid, the air turbulence will stabalize and be in a statistical form which will not cause erratic pressure readings. One can argue that the ensuing ventilating systern will so modify fan noise power output and its spectrum that it wili be unrecognizable at the system outlet, rendering accurate determination of fan noise output unnecessary. Perhaps, but how can 
fan noise laws, stanciardization, noise reduction calculations and design improvernents be possible without first having trusted and accepted knowledge of the acoustic characteristics of the source?

The matchec acoustic waveguide is hypothetically the simplest of acoustic systeins, yet is not without fault or complexity because of the 2000 to 1 frequency ratio of audible sound to be measured. To expect such wideband transmission in the analogous field of electromagnetic energy propagation in bounded waveguides would be ridiculous. The important difference in these analogous fields, aside from the nature of the energy itself, is that the free space acoustical plane wave will propagate down a tube at all frequencies, whereas the firee space electromagnetic mode (T.E.A.) will not. This is due to the boundary conditions allowing a wave with longitudinal variations, but not mutually transverse $\in$ lectric and inagnetic variations, to propagate. The plane wave becones the $(-1,0)$ mode of propagation as c'iscussed in Art. 2.1.3.

Wideband transmission of acoustic energy in pure plane wave form in a duct is limited at upper frequencies by the duct diameter (i.e. cut-ofi frequency of the lowest high - order mode) and at its lower extreme by the transforiter (horn) used to match the duct to its "load". These two problems are now considered in more detail.

2.1.2 LOW FRIOUEMCIES

Nuch of the noise output of fans and air turbulence is in the low frequencies (eg. $20 \mathrm{c.p.s}$. to $300 \mathrm{c.p.s}$.$) . The sys-$ tem of Fig. 1.3 is detailed in the section on Apparatus. It is 
similar to that developed by Beranek, Reynolds and Wiison!, with some modifications and with dimensions standardized to N.A.F.H. Its low frequency response is illustrated in terms of voltage standing wave ratio (V.S.M.R.) in Fig. 2.2(a). Its performance is acceptable for the most part down to $75 \mathrm{c.p} . \mathrm{s}$. What should be evaluated is the region below 75 c.p.s., in order to determine whether or not the observed reacings from $20 \mathrm{c.p} . \mathrm{s}$. to $75 \mathrm{c.p} . \mathrm{s}$. should be discarded. Four things cast doubt on these readings:

1. The horn cut - off is about 70 c.p.s.

2. The absorbing fiberglas wedges in the ternination cut off near $90 \mathrm{c.p.s}$.

3. The termination and duct eventually become on the same order of length as the sound wavelength $(\lambda)$ of interest. 4. The system walls cannot be regarded as entirely rigid. THE HORN

A horn (flared transmission line) is an acoustic transformer of analogous impedance ratio $A_{l} / A_{0}$, where $A_{0}$ is the throat area and $A_{l}$ the mouth area. The concentrated sound waves in the duct are gradually spread over a sufficiently large area so that they can continue with littie or no reflection. Response at low frequencies is very sensitive to the horn shape and the rate of increase of horn area. Unlike the electrical transforner, the analysis of the acoustic horn is extremely complex. A horn in the limit is simply a continuation of the duct (transforination of unity) or an infinite flange at the duct end (transformation of infinity). What is usually cesired is to transforn from the analogous duct characteristic impedance $\rho_{0} C / A_{d}\left(A_{d}=\right.$ duct cross sectional area) to that for free space, $\rho_{0} c$. Free space in the acoustic sense is not always possible to find 
Fig. 2.1

Calculated Throat Impedance of Modified Exponential Horn of FIG.3.5Below CUT-OfF
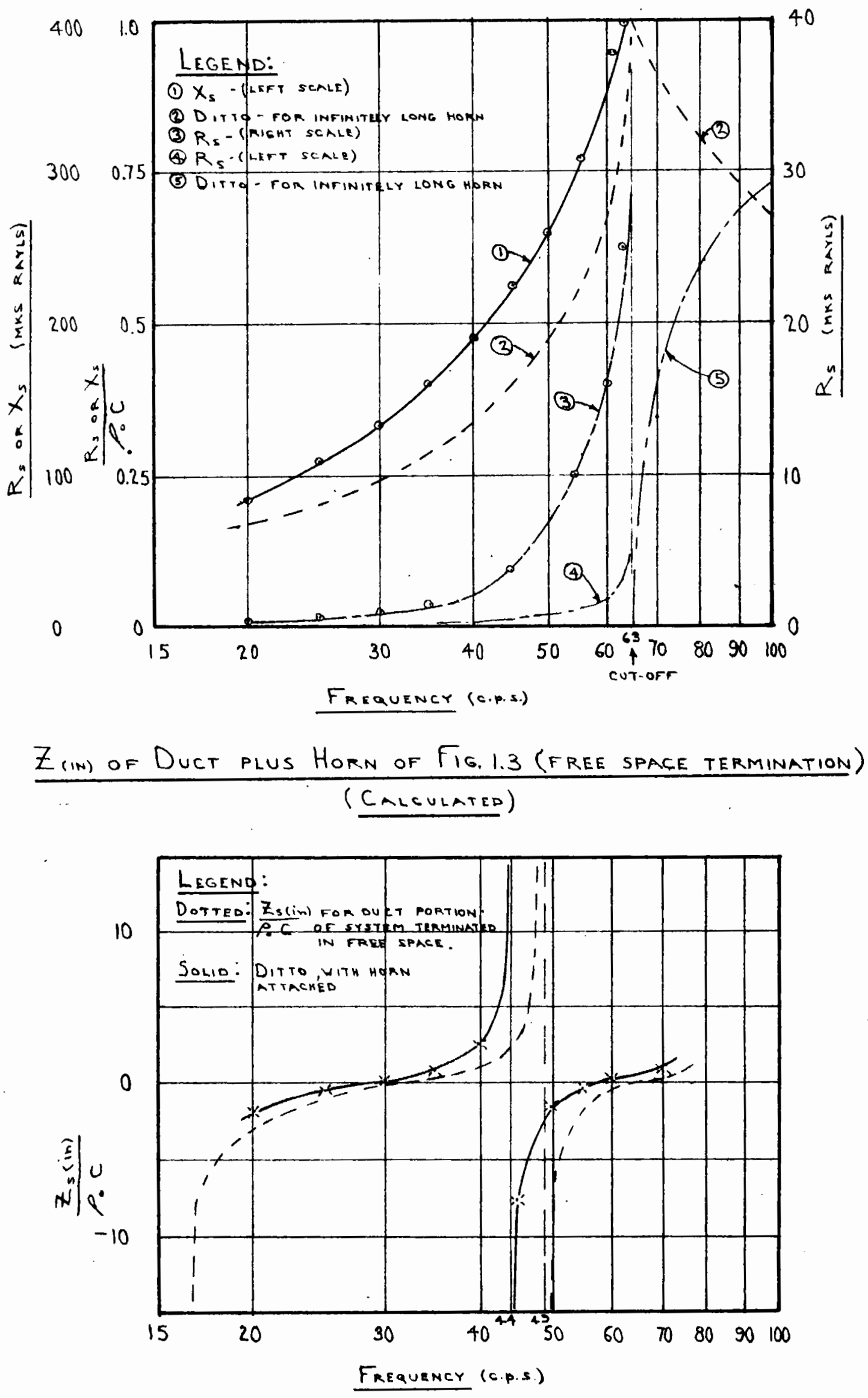
near the point of testing. The solution is to terminate the horn in a resistive termination consisting of a highly absorbent duct longer than the longest wavelength of sound of interest $(\lambda \max$.$) , and of analogous characteristic impedance P_{0} / A_{l}$ Assuming a perfect matched resistive load for the horn, the only limiting factors are the flare constant of the horn and its length. (Perimeter of mouth should be $>\lambda$ ).

of the four horn shapes often used: conical, exponential, catenoidal and hyperbolic; the exponential horn gives the most generally desired transmission - frequency characteristic. It is unity almost to cut - off. However, only the catenoidal horn will join smoothly to a uniform duct. The discontinuity of slope between throat and duct of an exponential horn is not severe if the horn is sufficiently long. Taking all these things into consideration, the modified exponential horn of Fig. 3.5 was designed and constructed by G. Chipps. Since no analysis of it was made, it was decided to compute its behaviour below cut off, considering it as a combination of an exponential and conical horn.

The throat reactance and resistance of an inifnitely long exponential horn of diminishing throat diameter is given by curves 2 and 5 respectively, Fig. 2.1. The computed reactance for the horm in Fig. 3.5 is given by curve 1 , and the resistance by curves 3 and 4 , for the region below cut - off. The expression used for this is due to Morse:

$$
\begin{aligned}
& \text { used for this is due to Morse: } \\
& \frac{Z_{s}(\text { throat })}{\rho_{0} c}=\left\{T \operatorname{coth}\left[\pi(\alpha-i \beta)-\left(\frac{i \omega \tau l}{c}\right)\right]+\frac{i c}{\omega h} \tanh (\epsilon)\right\}^{-1}(2.1) \\
& \pi(\alpha-i \beta)=\tanh ^{-1}\left\{\tau\left[1-2 \frac{J_{1}(w)}{w}\right]-i M(w)\right\} \cdot \cdot(2.2)
\end{aligned}
$$


$T=$ transmission coefficient

$$
=i\left(\frac{c}{\omega h}\right) \sqrt{1-\left(\frac{\omega h}{c}\right)^{2}}
$$

$(\alpha-i \beta)$ is a symbol used for convenience to transfer from $E q \cdot(2.2)$ to $(2.1)$

$h=$ flare constant for the horn (eg. for the exponential horn it is the distarice in which the cross - sectional area increases by a factor of $\epsilon=2.718$ ).

$W=\frac{2 \omega a}{c}$

$a=$ equivalent radius of the horn mouth.

$1=$ horn length.

$J_{1}(W)=$ Bessel function of first kind and order.

$M(W)=$ acoustic reactance ratio for an open ended tube (Table Vill, Morse).

$i=-j, i^{2}=j^{2}=1$

Other symbols as per glossary of symbols.

The calculated results in Fig. 2.1 show this horn to have a some what higher reactance below cut - off than an infinite horn, and also some resistance, which is very small but nonetheless shows some transmission of sub cut - off frequencies is possible. Free space termination is assumed.

The effect of this horn added to the open duct of Fig. 1.3 is to lower the resonant frequencies as shown in Fig.2.1 (bottom). This calculation was made from the familiar lossless line formula

$$
z_{\text {in }}=z_{0}\left[\frac{z_{L}+j z_{L} \tan \beta l}{z_{L}+j z_{L} \tan \beta l}\right] \cdot \cdot \cdot(2.3)
$$

$Z_{L}=$ throat impedance of horn terminated in free space as computed from eq. 2.1 
$z_{0}=$ free space impedance $\rho_{0} c=$ duct characteristic acoustic impedance for plane waves.

$$
\beta=2 \pi / \lambda
$$

THE "LOAL"

This horn transformed from an analogous electrical impedance of $P_{0} C / A_{d}=4000$ units to an impedance of $P_{0} C / A_{l}=370$ units. These are respectively the analogous ciaracteristic impedances of the circular duct and the terminating chamber. A terminating chamber was employed instead of free space because the latter could not be obtained in the area available for this research. The main absorption or "resistance" provided by this chamber is due to 16 wedges (described in Art.3) having a cut off frequency near $90 \mathrm{c.p.s}$. and having $30 \mathrm{sq}$. meters of exposed surface. Below cut - off, the termination becomes a "lossy" chamber or transmission line of length comparable to a wavelength. It thus exnibits resonances of its own. When the back pressure control panels are open the circuit takes the form shown in Fig. 2.3 (a). The holes act as:a small resistance and inductance in series, $(0.3+j 0.3)$ mks rayls at $20 \mathrm{c.p.s}$, holes completely open, (Ref. Beranek.p 138); a misinatch between the drilled panels and free space; and finally a sriall resistance and capacity in series, corresponding to a plasterboard wall 1.5 feet behind the back panels. The presence of the wedges within the termination causes a restriction and consequent reflection and some series inductance followed by the capacity of the rear charber.

When the back panels are closed for maximum back pressure, the termination may be regarded at low frequencies roughly as a closea box with a fiberglas lining. Its input impedance va- 
ries roughly from $1-j 8 \mathrm{mks}$ rayls at $75 \mathrm{c.p.s}$. to 2.5 - j320 mks rayls at $20 \mathrm{c.p.s.}(\mathrm{pp} 217,8 \text { Beranek })^{57}$. The equivalent circuit is shown in Fig. 2.3 (b). The back panel is actually a capacitor and radiator because $\frac{1}{2} "$ plywood is not perfectiy rigid at these frequencies.

\section{NON RIGID WALLS}

The duct is mastic coated 24 ga. U.S.S. (approx 0.0331 ) galvanized iron. The hom is $\frac{b}{r}$ and $\frac{1}{2}$ inch fiberglas - lined, mastic coated plywood. The termination has walls of $\frac{1}{4}$ inch braced, fiberglas - lined plywood. None of these constructions are rigid at low frequencies. Each can be likened to a capacitive reactance ( $\in g$. stiffiness controlled) and a radiation resistance in series. The wave is really no longer plane but exinibits the characteristics exnibitad in Fig. 2.2 (c). (There is a similar, but in this case negligible, effect due to viscous friction and heat conduction; ref. reston 41 ). The damping of the wave is proportional to the acoustic conductance ratio of the walls, the duct circunference, and inversely proportional to the cross sectional area. Jalls covered with a porous material add an additional damping factor. The equivalent circuit of such a wall bounding a propagating plane wave is given in Fig. 2.2 (d) (ref. rorse 56

\section{LON FREUTHCY TRANSMISSION}

The indivilual elements influencing low freguency transmission of this acoustic waveguice have been qualitativeiy analysed. The low frequency behaviour of the system is roughly approximated as the lumped element circuits shown in Fig. $2.3(a)$. All resistances and reactances are frequency depeident, and there 
(a) Performance. of Acoustic Waveguide shown in Fig.1.3

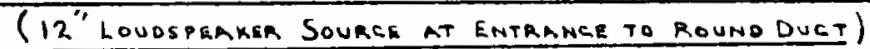

VoLTAGE SWR. (db)

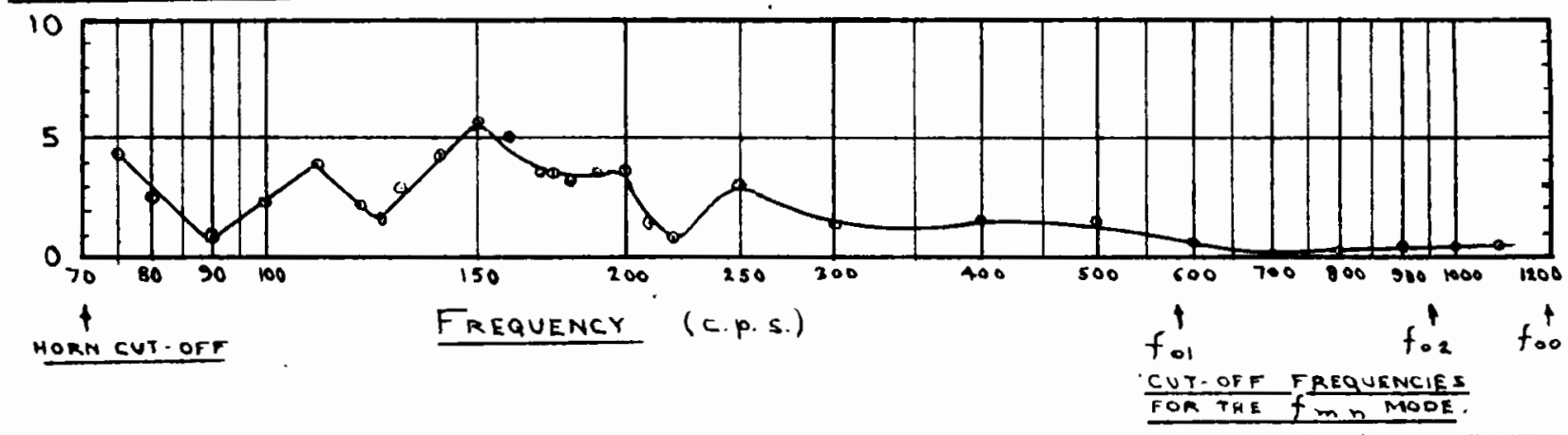

(b) Low Frequency EfFEct of Junction at RIGHT
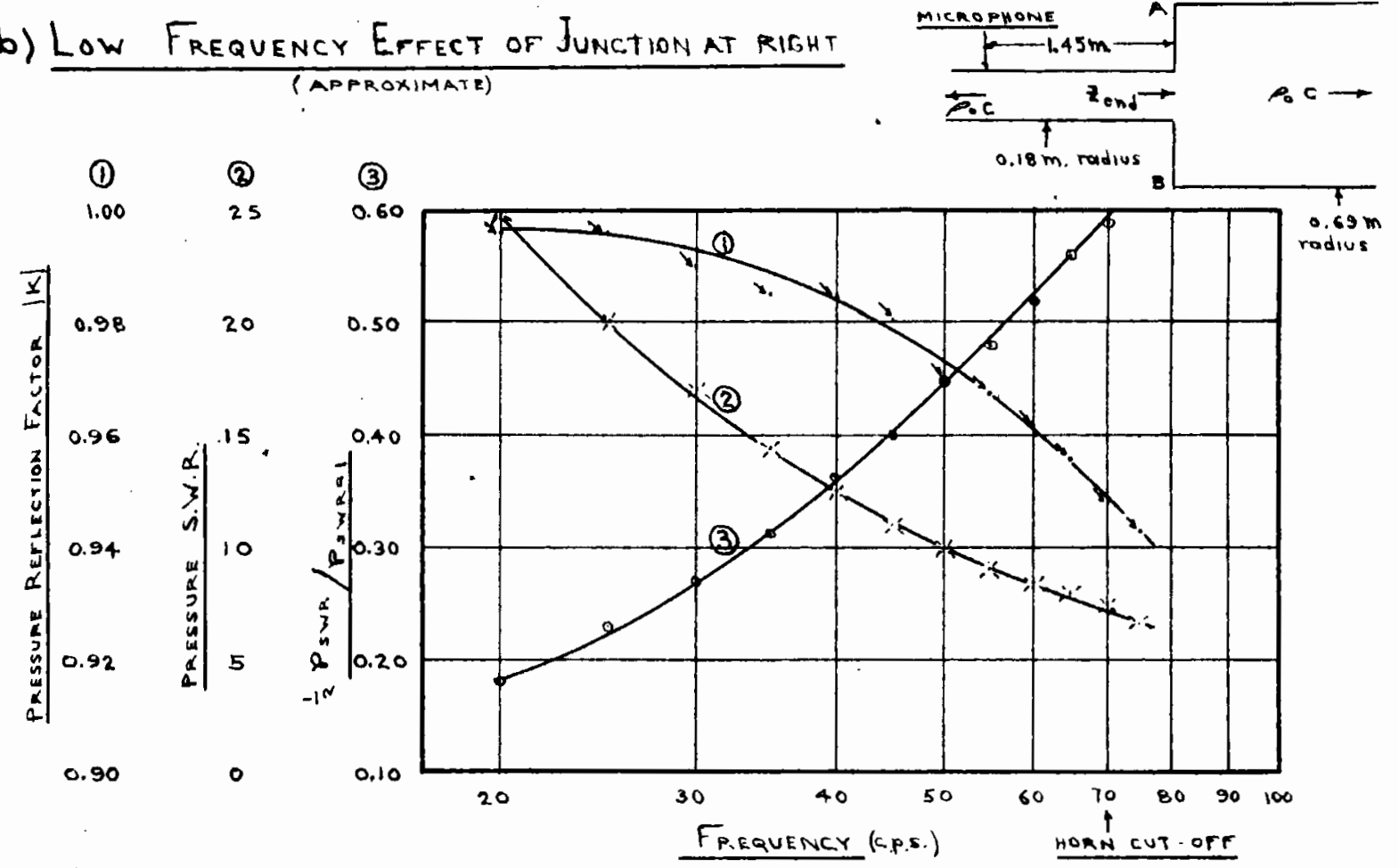

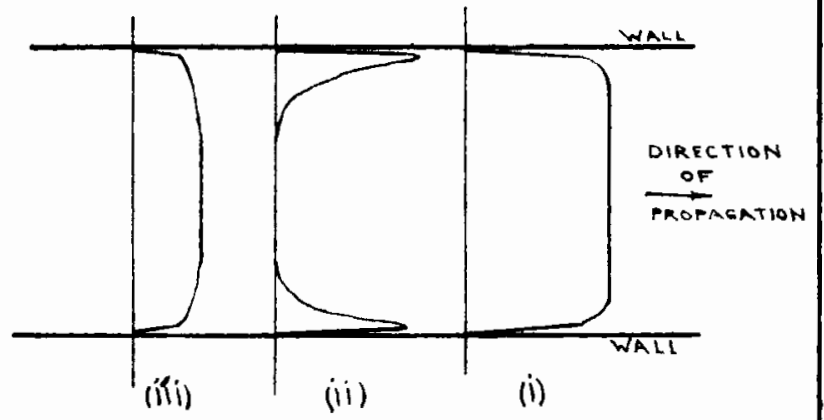

(i) AMPLITUDE OF AXIAL PARTIGLE VELOCITY

(ii) Amplitude of rudial particle velocity (iii) WAVE FrONT OF Equiphase surFace

(c) Effect of Wall Motion and $\frac{\text { Viscosity on Plane Wave ina Duct }}{\text { (arter. Weston })^{41}}$

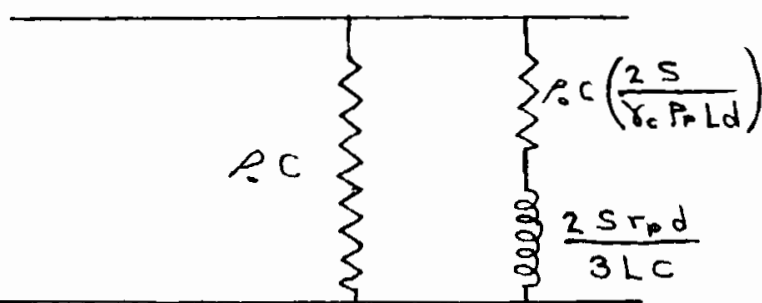

$S=$ DUCT ARER

$L=$ DUCT PERIMETER

$d=$ THICKNESS OF POROUS MATERLAL

$\gamma_{c}=$ RATIO OF SPECIFIC HEATS = 1.4 FOMAIR

$Y_{P}=$ FLOW RESISTIVITY OF POROUS MATERIAL

PF = PUROSITY OF POROUS MATERIAL

DENSITY OF THE FOROUS MATERIAL ASSUMED NEGHGIBLE

(d) Equivalent Circuit of a Dult LINED WITH a POROUS MATERIAL (AFTER Monst) 
Equivalent Electrical Circuitry of Acoustic Waveguide SYSTEM OF FIG. 1.3

(a) LOW FrequenCIES

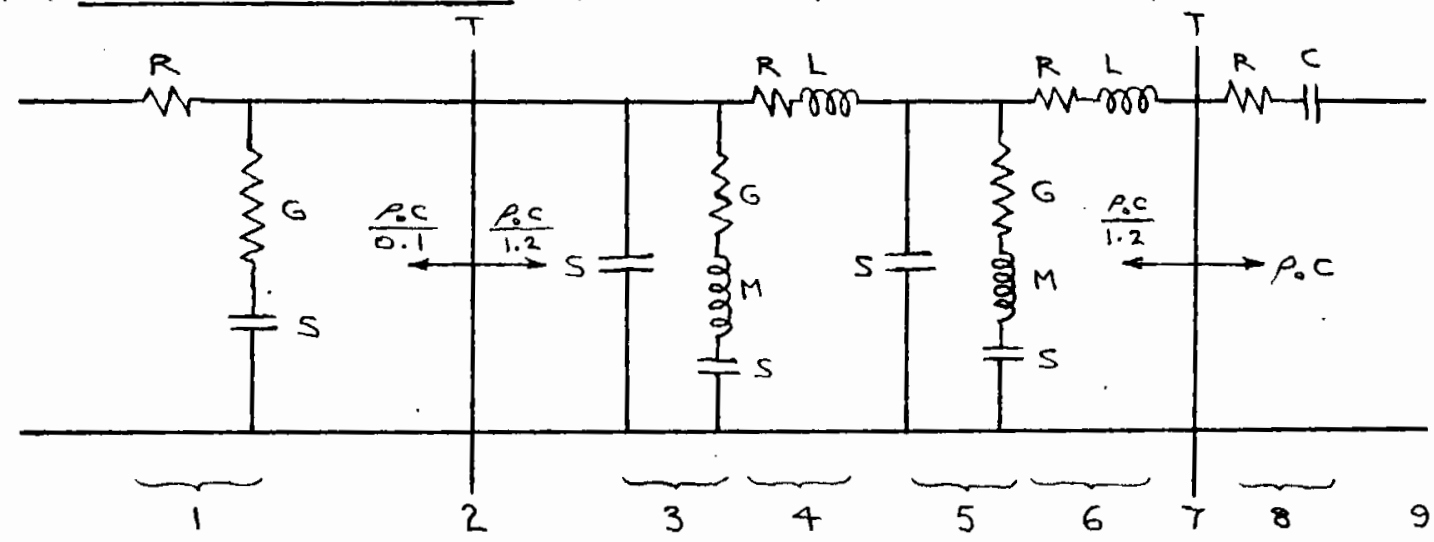

Fig. a LeGEND:

I Circular duct

2 Duet - Horn-Termination Mismateh

3 Termination up to weoges

4 WEDOES

5 Termination after weoges

6 Adjustaele reak manels, hOLEs open

7 Mismatch aetweem termination a pree space

8 PlasterbonRo Wall $11 / 2$ Behind termination

9 Large adjoIning moom
ELEMENT LEGEND (AL FigURES)

R: series resistance - viscous loss OVERALL EFFELT VERY SMALL

L: SERIES INDUSTANCE - DUE TO RESTRICTED marea

C: SERIES capacity - DUE to memarane of , rinite impedace across patu.

G: CONDUCTRNCE - DUE TO ABSORPTION AND RADIMTIOH.

M: ShUNT inDUETAKCE DUE to AIR MOTION IN AND OUT OF PORES OF MASOMEENT LINING - VERY SMALL ETFECT HERE.

S: SHUNT CAPACTANCE DUR TO SUDDEM EXPANSAON INTO A VOLUME AND MLSO DUE TO FINITE. STIFFNESS OF DUCT WALLS.

T: ImFEDANCE MISMATCH DUE TO DISCONTINUITY (b) Low Frequency Simplification of (d) When ReAr PAMels are Closed

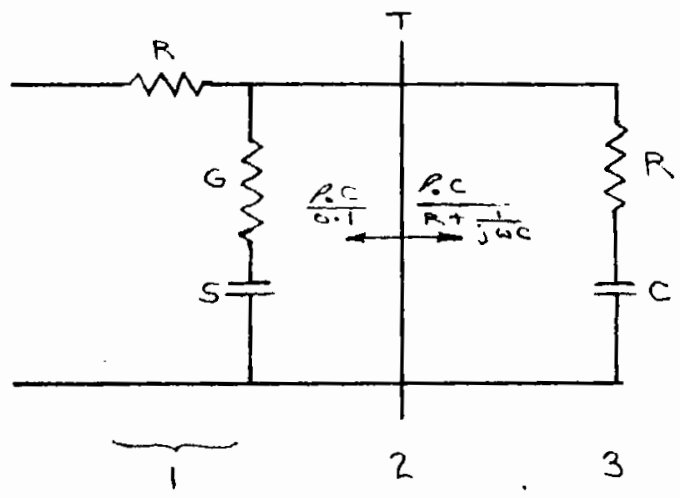

1,2 As in (a)

3 Termination treated as a MEDIUM SIZED CLOSED LINED BOX.
(C) High Frequency Circuit

(GENERALIY APRLICABLE ABOVE HORN SUT-OFF)

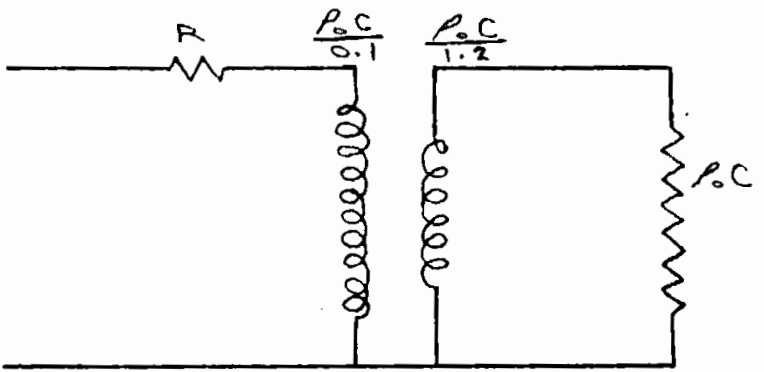

12

3

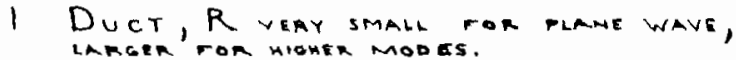

2 Modificd ExPONENTIAL MORN

3 Termination 
are many unknowns such as the stiffness factor of materials composing the systems. It would seem that below cut - off of the horn, the circuit behaves fundamentally as a trarsmission Iine on the order of a wavelength long. It has a composite structure causing impedance mismatch and is ultimately capable of radiating some energy, transmitting some, absorbing some and hoarding the remainder.

\section{Below cut - off then, heterogeneous transmission can} be expected through the system. The standing wave ratio could not be measured below 75 c.p.s. (see Art.3). Although the transmission curve in this region is low and has peaks and valleys, there are several reasons for believing that the error in the 20-75 c.p.s. band is not as phenomenal as the above would indicate:

1. If noise spectra is fairly uniform in this band, and the position of the micropione is constant, it will sample energy from various positions on a standing wave, depending on the frequency. To evaluate this roughly, the horn as assumed to be a simple discontinuity between two ducts of area 0.1 sq.meters and $1.16 \mathrm{sq}$.meters. This is a fair approximation when the horn length $<\lambda / 2$. The second cuct was assumed to be infinitely long. Although this is far from true, the amount of energy transmitted into this duct and reflected back is trifling, hence the net effect on the standing wave of energy within the 0.1 sq.meter duct can be considered as negligible. The specific acoustic impedance at the open end of a tube fitted with an infinite flange is given by 
Morse:

$$
z_{\text {end }}=\rho_{0} c\left[1-2 \frac{J_{1}(w)}{w}\right]+j \rho_{0} c M(w) \cdots(2.4)
$$

where the symbols are listed after eq.(2.2).

When $\lambda>8 \pi \alpha$, as is the case for the $14^{\prime \prime}$ duct in

question below 75 c.p.s., $(2.4)$ becomes

$z_{\text {end }}=\frac{\rho_{0} a^{2} \omega^{2}}{2 c}+j \omega\left(\frac{8 \rho_{0} a}{3 \pi}\right) . \quad . \quad$ (2.5)

"a" being the tube radius.

If instead of an infinite flange there is a discontinuity such as we have assumed, it may to a rough approximation be considered as a flange less than a wavelength in size, in which case eq. (2.5) becomes

$$
\begin{array}{r}
z_{\text {end }}=\frac{\rho_{0} \alpha^{2} \omega^{2}}{2 c}+j \omega\left(0.6 \rho_{0} a\right) \\
\text { (ref. Morse) }
\end{array}
$$

Using common relations for the short electrical transmission line:

Pressure reflection factor $K=|K| \epsilon^{j \psi}$

$$
\begin{aligned}
& \frac{p_{r}}{p_{i}}=\frac{z_{2}-z_{1}}{z_{2}+z_{1}}=k \\
& \left(\beta y-\frac{\psi}{2}\right)=(2 h+1) \frac{\pi}{2}
\end{aligned}
$$

$z_{2}=$ impedance seen from the tube end (eg.eq.2.6)

$z_{1}=P \cdot c$

$|k|=$ magnitude of $K$

$\psi=$ phase angle of $K$ in radians

$\beta=2 \pi / \lambda$

$y=$ distance from tube end (discontinuity) that pressure minima occur

$h=$ positive integer or zero 
$P_{r}=$ magnitude of the reflected pressure wave

$P_{i}=$ magnitude of the incident pressure wave. $|k|$, S.W.R. $=\frac{z_{2}-z_{1}}{z_{2}+z_{1}}, y_{\text {min }}(n=0)$ were calculated at each 5 c.p.s. interval from 20 c.p.s. to 75 c.p.s. Knowing the position of the pressure minimum and that the microphone position was 1.45 meters from the discontinuity, and further assuming a standing wave shape of rectified sine waves, the relative reading was computed. This is approximately the ratio of the reading at the microphone due to the standing wave $P_{s w \mathbb{R}}$ to the reading which would be obtained if S.W.R. was unity $\left(P_{1}\right),|K|$, S.W.R. and $\frac{1}{2} P_{s w r} / P_{1}$ are plotted in Fig.2.2(b). The average $\frac{P_{\text {swe }}}{P_{1}}=0.83$. The microphone was therefore in such a position as to give a fair average of S.P.L. assuming a uniform spectrum of sound energy from 20 - 75 c.p.s. It is at approximately the maximum upstream position for measurement in stable turbulence (see Art.3). Experimental values of $y_{\text {min }}$ were checked at 75,50 and 35 c.p.s. as described in Art. 3. Agreement with the calculated values was moderately good.

2. The small sub cut-off horm transmissibility and radiation loss help to moderate the value of S.W.R. in the duct.

3. G. Chipps7 found:

(a) Negligible 20 - 75 c.p.s. band S.W.R. when the fan was used as a source.

(b) Variations of $2 \mathrm{db}$ in the $20-75$ c.p.s. band when the termination was removed from the duct.

(c) Fan noise attenuation through the termination was 
about $20 \mathrm{db}$ in the $20-75 \mathrm{c} . \mathrm{p} . \mathrm{s}$, band, increasing to $33 \mathrm{db}$ in the $4800-10,000 \mathrm{c} \cdot \mathrm{p} . \mathrm{s}$. band.

(d) "Indications are that the termination does not have too much effect if a fixed microphone position is used."

The above was generally verified by the writer.

\subsubsection{HIGH FREQUENCIES}

Above horn cut - of $f$, the transmission coefficient rises rapidly and standing waves in the duct drop to a tolerable level. The plane wave of sound is essentially matched from 75 c.p.s. to 1200 c.p.s. as seen by Fig.2.2(a). Above 1200 c.p.s. it becomes impossible to measure S.wR. due to higher order modes masking the plane wave. The higher frequencies are matched once they reach the horn, but there is considerable non-uniformity of energy both axially and radially in the duct as shall be seen in Art. 3 .

Acoustical propagation of higher order modes is directIy analogous to that of electromagnetic energy propagating down a hollow uniform waveguide. Assuming a uniform, smooth, rigid tube of inner radius a, the wave equation in cylindrical co ordinates for excess pressure is:

$$
\begin{aligned}
& \frac{1}{r} \frac{\partial}{\partial r}\left(r \frac{\partial p}{\partial r}\right)+\frac{1}{r^{2}} \frac{\partial^{2} p}{\partial \theta^{2}}+\frac{\partial^{2} p}{\partial z^{2}}=\frac{1}{c^{2}} \frac{\partial^{2} p}{\partial t^{2}} \cdot(2.10) \\
& \text { of which the solution is the sum of terms of type: } \\
& J_{m}\left(k_{c} r\right)[A \cos m \theta+B \sin m \theta] \sin k_{c} t \cdot \cdot(2 \cdot 11) \\
& r=\text { radial distance from pipe axis } \\
& k=\text { wave number }=\omega / c
\end{aligned}
$$


$J_{m}\left(k_{c} r\right)=$ Bessel function of first kind, and of order $\mathrm{n}=0,1,2$ etc.

Applying the boundary condition that particle velocity at $(r=a)=0$, then $\frac{\partial p}{\partial r}=0$ and $J_{m}^{\prime}\left(k_{c} \alpha\right)=0$. The cut - of $f$ wave number $k_{c}$ can have only values that allow $\left(k_{c} a\right)$ to be a root of $J_{m}^{\prime}\left(k_{c} a\right)=0$. If $\chi_{h}$ is the $n$th root of the Bessel function, $J_{m}(x)=0$, then

Higher order mode notation follows as $(\mathrm{kr})_{\mathrm{m}, \mathrm{h}}$, or the $\mathrm{m}, \mathrm{n}^{\text {th }}$ mode where

$$
\begin{aligned}
& m= \text { number of cycles of space variations of pressure } \\
& \text { on a circle concentric with the axis of tine tube. } \\
& n= \text { number of concentric cylinders on which the axial } \\
& \text { component of pressure vanishes. }
\end{aligned}
$$

An excellent reference for sketches of mode configurations is Microwave Theory and Techniques ${ }^{58}(p .265-279)$. The analogy is the transverse electric configuration, substituting equal pressure lines for electric field lines and instantaneous particle velocity direction for magnetic field lines. If this is done, a TEm, $n$ mode is aralogous to a $(\mathrm{kr}) \mathrm{m},(\mathrm{n}-1)$ acoustic mode. This analogy is true for pipe cross sections, but is not applicable to longitudinal sections.

For any particular tube radius a and sound velocity $c$,

$$
f_{m, n}=\frac{(k \alpha)_{m, n} c}{2 \pi \alpha}
$$

from which we can compute the cut - off frequency for any desired mode. A tabie of the lower modes follows; 


\section{TABIE 2.1}

\begin{tabular}{|c|c|c|c|}
\hline Mode & f c.p.s. & Mode & f c.p.s. \\
\hline $\begin{array}{l}01 \\
02 \\
00 \\
03\end{array}$ & $\begin{array}{r}580 \\
960 \\
1200 \\
1320\end{array}$ & $\begin{array}{l}11 \\
12 \\
10 \\
13\end{array}$ & $\begin{array}{l}1670 \\
2110 \\
2205 \\
2520\end{array}$ \\
\hline
\end{tabular}

It is the 00 mode that upsets the measurement of S.r.R. near 1200 c.p.s. It possesses both axial and radial particle velocity, and longitudinal pressure variations on both axis and along the circumference. Placing an interior flange inside the duct failed to suppress it, however, such a meais would cause inismatch. Attempts to neasure relative radial to longitudinal particle velocity using a technique suggested by Lambert 42 iailed due to an inadequately constructed detector. The only useful information it revedied was that radial particle velocity did exist frow about 600 c.p.s. up.

Fortunately, the declining spectrun of fan noise renders errors in overall noise levels due to the presence of higher order nodes negligible. a tecinique outlined in art. 3 discusses bandwise correction due to rauial variations at the higher frequencies.

\subsection{FAN AIND TURBULENCE AS A NOISF SUURCE}

There are two general aethods of creating contruiled air flow; positive displactaent, as occurs when a piston noves in a cylinder (air conpressor); and inúucea motion, as occurs when a blade rotates ( $\hat{f} a n)$. The former is most used for high 
pressure power and control applications, the latter for uloving air for vertilating purposes.

A fan must contain a noving (usualiy rotating) element which may be surrounded with an energy converting casing. Consider the simplest case; a single rod being rotated in space about its center. Tnere are three distinct sources of noise created by such motion, quite apart from any vibration which is here assumed $z \in r o$; :

(1) A "rotational" or "blade passage" coinponent and its harmonics. This results from positive displaceneni of the air caused by the finite thickness of the whiriing elenent. Regular pulses of air emanate froin the source. The phasing is such that it is a maximum in the plane of rotation and theoretically zero on the axis of rotation. For the simplest profile, a cylindrical rod, acoustic power output of tilis noise increases as the 5.5 power of the rotational speed. The magnitude depends on the rod diameter and tip speed (ref.13).

(2) A "profile" noise due to a scale of turbulence established by the rotating eleinent. This may possess some regularity associated with a regular train of generated vortices, or may be purely of a statistical randoin nature covering a wide band of frequencies. In any case it is unique for a particuiar profile, its speed and environment. This type of noise appears to increase as the 6 power of rotational speed. (ref.34,35).

(3) "Aerodynamic" noise which is due to turbulence of the 
fluid medium itself. Quite apart from the noise generated in establishing this turbulence, once it is set up, it generates a wide band of noise that increases as che 8 power of a typical flow in the turbulent medium (ref. 18 ).

Each of the above processes is less efficient than the preceeding one. In the study of ventilating equipment and air motion, (3) is not significant compared to (1) and (2). AII of these methods of noise production are loosely classified as "aerodynamic", although strictly speaking, only (3) deserves this title since only (3) is generated without the aid of solid boundaries. In this thesis, the above sources will be classified as (1) Blade component, (2) Boundary turbulence noise and (3) Self turbulence noise. Iighthill has shown (3) to be equivalent to a distribution of quadrupoles oriented throughout the turbulent volume. One and (2) are acoustically equivalent to a dipole distribution along the profile, or covering the boundary surface. A fourth type of generation is due to vibration of a solid member in air. Any rotating system contains ail of these sources of noise generation to a certain degree. We shall now narrow the discussion to the type of rotating system represented by that class of fans called Centrifugal. The ducted centrifugal fan is now discussed in light of recent theory. 


\subsubsection{EXPECTED TURBULENCE}

Air is rarely quiescent. Aside from the molecular motion due to the energy level at which it is, there is nearly always a net flow. In nature the ultimate source of both the energy state and flow is the sun's energy spectrum reacting on a boundary, the earth's surface. Wulti directional air flows are manifested. Such motion has many properties; it can be "felt", "seen", heard (aerodynamic souna); it evaporates liquid, suspends particles, scatters electromagnetic energy, and so on. It is not possible to either move air, or move an object through it at an appreciable velocity without causing turbulence of the air. Ventilation involves both situations; moving solid boundaries through air, and moving air itself.

We may visualize the action of the rotor in a centrifugal fan in two ways; either as wide cups or blade tips on the periphery of a disc, or as curved or flat plates moving through the air in such a way that the motion is perpenaicular to their length. Let us examine a single profile in the form of a long plate moving rapidy through the air. This is somewhat, though not exactly, analogous to air moving swiftly past a stationary blunt object, since in this latter case the air is turbulent prior to reaching the object.

A blunt profile moving through quiescent air will have a hypothetical wake made up of a fully turbulent core bounded by intermittent turbulence as shown in Fig.2.4(b) (Ref.43). This idea is plain if one observes the wake of an object moving slightly submerged through quiescent water. The picture one 
(a) Typical Time Variation of Fluid Property ina Turgulent Flow

$u$

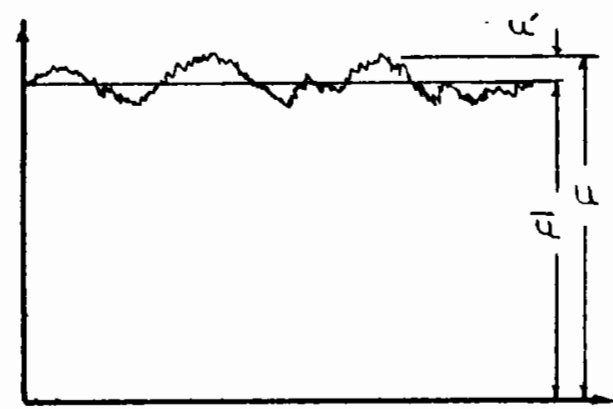

Time (b) Section of HYpothetical WaKe STRUCTURE 4.3 (ATtan TOWNSEND)

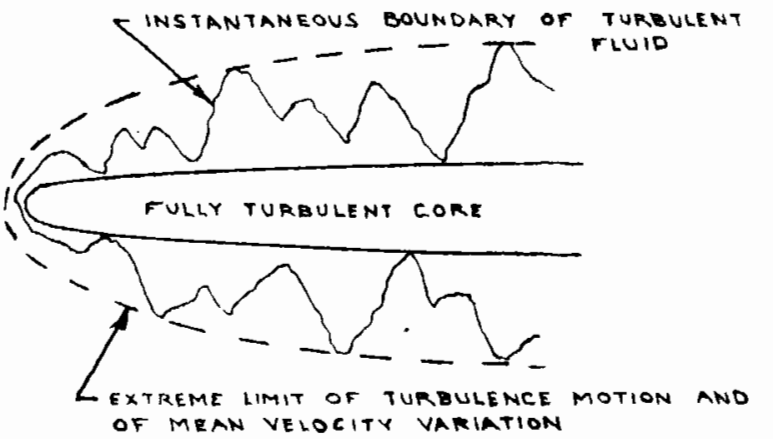

(C) Illustration of the Momentum Equation (laminar boundary layer)

FORCES

$\mu \frac{\partial u}{\partial y} d x+\frac{\partial}{\partial y}\left(\mu \frac{\partial u x}{\partial y} d x\right) d y$

$r--\overrightarrow{-}-7$

$\rho d y \rightarrow \begin{array}{lll}1 & 1 \\ 1 & \text { contmol } & 1 p d y+ \\ \text { volume } & 1 & \frac{\partial}{\partial x}(p d y) d x\end{array}$

$d y+\frac{1}{L}-\ldots-\ldots$

$\overrightarrow{d x} \quad \overrightarrow{T_{x y} d x}=\mu \frac{\partial u_{x}}{\partial y} d x$
MOMENTUM FIUXES in $x$-DIRECTION

$u_{y} u_{x} d x+\frac{\partial}{\partial y}\left(\rho u_{y} u_{x} d x\right) d y$

$1--\sqrt{-}-7$

$\rho \mu^{2} d y \frac{1}{1} \underset{\substack{\text { controu } \\ \text { Voumz }}}{\frac{1}{1} \rho u^{2} d y t}$ $d y+1$

$\int \frac{\partial}{\partial x}\left(p u^{2} d y\right) d x$

$\overrightarrow{d x} \rho u_{y} u_{x} d x$.

T= SheAr stress actine on ay face in the $x$ DIREction SEE ART.2.2.2 FOR OTHER SYMBOLS

(d) Experimental Spectrum of TURBULENCE

$F(t)$

$0.2+U=$ WIND TUNNEL AIR SPLED

- $-U=15$ +\%se. SPEED $U=30^{4+/ \sec }$.

0.015

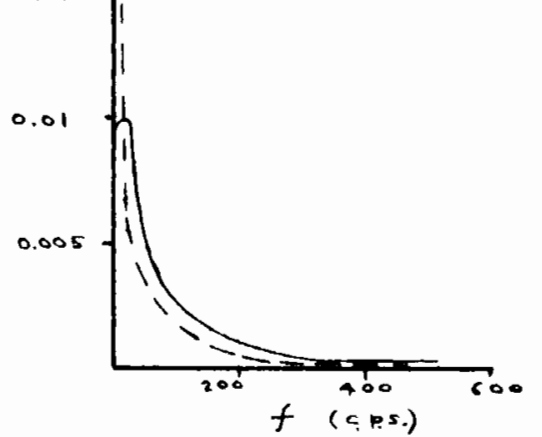

$F(t)=$ STATISTICAL FREQUENCY FUNCTION DENOTINC THE PROBABILITY OF THE EXISTENCE OF TURBULENCE VELOCITIES mitwen $f$ ANO $t+d f$.

HOT-WIRE ANEMOMETIR, FITER TECHIIQUE (AFTER SIMMIONS \& 23 (e) Line Components of Noise Produced By a Centrifugal Fan

d - CAM- PUMP ACTION OF CGCENTRIS RUNNER IN PRODUCING AIR PULSEX.

b - HOUSING MOVEMENT DUE TO DYNAMIC RUNNER UNEALANCE MOVES MASSES OF MIR LIKE $A$ LOUDSPIAKER DIRPHPAM AT LOW FREQUENCIES.

C- BLADE COMPONENT DUE TO BLAOES PASSING "CUTOF" POINT.
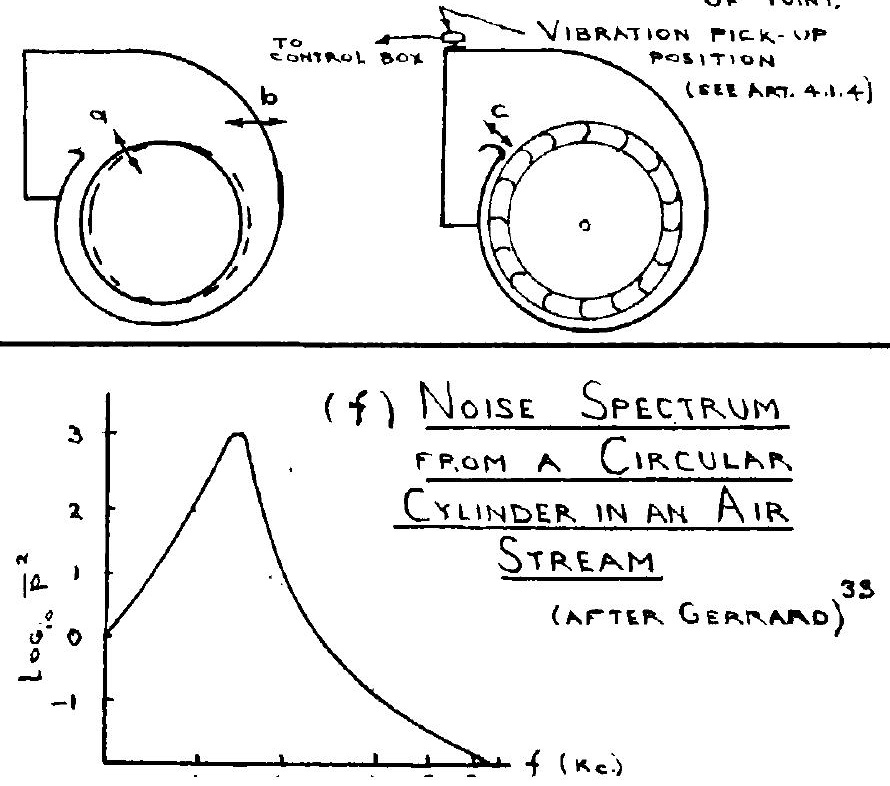
obtains on the water surface can be roughly applied to a Schlieren photograph of the turbulence within a cross section of the wake shown in Fig.2.4(b). Where does the energy applied to the profile ultimately go? Most of it eventually heats the air, as shall be seen shortiy, and an extremely small fraction emanates as sound which ultimately is dissipated in space by heating the air. The spectrum of noise is dependent primarily on the velocity of the object.

Let the object be stationary and air flow past it. Fig.2.4(b) still applies, only now all the air is filled with turbulence, and the blunt profile introduces another scale of turbulence which will gradually fuse with the ainbient turbulence as the distance downstream increases. For the case of a circular cylinder in an airstream, Gerrard 33 found that three distinct types of flow exist in the wake of a rigid boay, and these depend on the Reynolds number of the flow (Rey., see glossary of symbols).

Rey $<50$ - no vortices are shed, flow is laminar. $50<$ Rey $<10^{5}$ - periodic shedding of vortices takes

Rey $>10^{5}$ - the periodic characteristics of the flow are replaced by the random fluctuations of turbulence.

To appreciate these values of Rey. number, a Rey of 50 corresponds roughly to an air speed of $\frac{1}{2} \mathrm{ft} / \mathrm{min}$ in the duct of Fig.1.3, while Rey of $10^{5}$ corresponds to about $850 \mathrm{it} / \mathrm{min}$. It is quite apparent then that most of the turbulence that occurs in this system will be random, and that laminar flow is never attained. Gerrard discovered that sound of relatively pure tone character is radiated for $50<$ Rey $<3 \times 10^{4}$ and that the 
frequency of vortex shedding from one side of the cylinder, and the acoustic frequency were equal. A typical spectrum is shown in Fig. 2.4(f).

We may then very well assume that in most instances, a random spectrum will emanate from the centrifugal fan rotor, and that the outlet stream is fully turbulent. The discussion is directed at centrifugal fan rotors of say a foot diameter and larger, so that relatively large scale, high Rey flow prevails.

\subsubsection{RANDOM TURBULENCE AND AERODYNAMIC NOISE}

Turbulence and turbulent flows are of great practical significance, yet they are not well understood theoretically, exact analytical description being well nigh hopeless. The large number of variables involved are reduced to a manageable form of statistics by making vast simplifications:

(1) The governing equations are written in terms of the time-mean fluid properties as though the flow were steady .

(2) Crude terms are then added to (1) to represent the influence of unsteadiness superposed on the time mean flow. For our purposes, one term will suffice, called somewhat misleadingly the "Reynolds stress" but better named "momentum flux tensor" $M_{x y}$. In turbulent flow, the effect of viscosity can be safely neglected.

This amounts to the physical picture of Fig.2.4(a). Defining the components: 


$$
\left.\begin{array}{ll}
u_{x} \equiv \bar{u}_{x}+u_{x}^{\prime} & \rho u_{x}=\overline{\rho u_{x}}+\left(\rho u_{x}\right)^{\prime} \\
u_{y} \equiv \bar{u}_{y}+u_{y}^{\prime} & \rho u_{y}=\overline{\rho u_{y}}+\left(\rho u_{y}\right)^{\prime} \\
\rho \equiv \bar{\rho}+\rho^{-} &
\end{array}\right\}
$$

$u=$ flow velocity

$\rho u=$ apparent flow ( $\rho=$ density $)$

$x=x$ direction

$y=-y$ direction

Bar over a symbol denotes the slowly varying temporal mean value.

Prime denotes instantaneous deviation.

If $t$ denotes time, then by definition

$\bar{u}_{x}=\frac{1}{t^{*}} \int_{0}^{t^{*}} u_{x} d t$, etc.

t large compared with the period of the fluctuations.

$\vec{u}_{x}=0$ (average time of any fluctuating component is $\frac{\partial \bar{u}_{x}}{\partial x}=\frac{\partial}{\partial x}(\bar{u})=\frac{\partial \bar{u}_{x}}{\partial x}$, etc.

Manipulating the continuity equation in time-mean form, substituting into the momentum equation and assuming a very thin boundary layer, a steady flow in the mean, and the time-mean viscous stress negligible, we end up with the desirable expression:

$$
\bar{\rho}_{x} \frac{\partial \bar{u}_{x}}{\partial x}+\overline{\rho u_{y}} \frac{\partial \bar{u}_{y}}{\partial y}=-\frac{\partial \bar{p}_{x x}}{\partial x}+\frac{\partial}{\partial y}\left[\overline{\left(\rho u_{y}\right)^{\prime} u_{x}}\right]
$$

which is the simplified momentum equation for turbulent flow in the $x$ direction.

$$
\begin{aligned}
p_{x x} \equiv \bar{p}_{x x}+p_{x x}^{\prime}= & \text { compressive stress tensor, representing } \\
& \text { the force in the } x \text { direction acting on } a \\
& \text { portion of the fluid, per unit surface } \\
& \text { area aligned normal to the } x \text { direction. }
\end{aligned}
$$


The forces and momentum fluxes involved in the unmodified momentum equation are illustrated in Fig. 2.4(c).

Eq. (2.15) contains the term of interest as far as we are concerned,

$$
M_{x y}=\frac{\partial}{\partial y}\left[-\overline{\left(\rho u_{y}\right)^{\prime} u_{x}}\right]
$$

$=$ Reynolds stress or momentum flux tensor (We will adopt the latter).

It is the time-mean of the $x$ - momentum flux crossing a surface aligned normal to the y direction. This momentum - exchange term accounts for the unsteadiness of the flow when the momentum equation is written in terms of the time-mean flow properties. There is a similar equation to (2.15) and term (2.16) corresponding to energy balance. They are respectively the "energy equation" and "heat flux tensor", but they do not enter the discussion on aerodynamic noise, although they are necessary to describe a turbulent field. An excellent development of this material is to be found in Shapiro's Vol II, of Compressible Fluid Flow.

Keeping in mind the vast simplifications which have been made regarding a turbulent stream we now turn to Lighthill's great contribution to aerodynamic noise.18,19 Eq.(2.15) expresses a turbulent field as though a fixed region of gas were at rest under the combined action of hydrostatic pressure and viscous stress (which was neglected) plus the fluctuating momentum flux "stress". A uniform acoustic medium at rest experiences stresses only in the form of a simple hydrostatic pressure field, whose variations are proportional to variations in density, with $c^{2}$ being the constant of proportionality. Therefore Lighthill postulates, the density fluctuations in the 
real flow must be exactly those which would occur in a uniform acoustic medium subject to an external stress system given by the difference:

$$
M_{x y}+p_{x x}-c^{2} \Delta \rho
$$

This incorporates:

(I) The generation of sound

(2) Its convection with the flow (in part of the term $\left.M_{x y}\right)$

(3) Its propagation with variable speed

(4) Gradual dissipation by conduction

(5) Gradual dissipation by viscosity.

In practice, Lighthill claims, only $M_{x y}$ is important to the generation of sound. By forcing the rates of momentum flux across fixed surfaces to vary, sound is generated aerodynamically with no motion of solid particles. The physical interpretation he applies is that any forcing motion on a scale comparable to a wavelength of sound of interest is balanced partly by a local reciprocating motion, or standing wave, and partly by compressions and rarefactions of the air whose effect is propagated outwards. The larger $\lambda$ in comparison with the scale of the forcing function, the more completely can the motion be fully reciprocated by the local standing wave. It is the radiation due to the minute fraction of the fluctuation in momentum flux which is not balanced by a local reciprocating action that Iighthill determines. Mathematically he finds, "the radiation field of a distribution of acoustic quadrupoles" (see Fig.1.5(d)).

Presumably, random turbulence will result in random noise of very wide spectrum. This is upheld by looking at 
Kolmogoroff's "Theory of Locally Isotropic Turbulence" 15. He postulates that turbulent flow consists of pulsations ranging in scale from a dimension characteristic of the mean flow (which may be non-uniform), to a lower limit at which the motion is entirely laminar.

Consider a high Rey. number flow. Such a flow is unstable to small disturbances hence it will always have superposed on it a set of pulsations or eddies which have characteristic dimensions and velocities one order lower than the corresponding quantities, characteristic of the mean flow. On this first order flow, which in turn is unstable at sufficiently high Rey. numbers, is superposed a set of pulsations or eddies of still smaller characteristic dimensions and velocities. This "eddy cascade" process is repeated until the Rey. number of the smallest pulsations is too small to permit the formation of smaller eddies. If the wavelength of an eddy is defined as its mean diameter, then in a turbulent flow we should expect wavelengths ranging from $\lambda_{1}$, of the largest eddies to $\lambda_{h}$ of the smallest eddies commensurate with the Rey. number of the flow. The larger the Rey., the larger is $n$. We may then expect wideband noise from Lighthill's considerations.

Most analyses assume turbulence is isotropic; that is the instantaneous motion of an infinitesimal volume of the turbulent motion is spacially random, superposed on a d.c. flow. There is a strong tendency to isotropy in an air flow downstream from a regular grid placed in series with the air flow 22 . This corresponds to the microphone location in Fig. 1.3. The turbulance at and near fan blades is obviously not of this 
type. However, if a small enough volume of turbulent air is considered, isotropy will exist in that volume. Hence behind a regular grid, eddying flow quickly transforms to isotropic flow and the volume considered can be quite large, within which isotropy is dominant. This implies we will get a random noise spectrum from the isotropic turbulence, plus a more unique and possibly narrower band spectrum from the larger scales of turbulence which are not isotropic and which are dependent to a degree on the boundaries (eg. fan blades) that produced them. In the extreme there are the aeolian, jet and edge tones which may be considered due to a dominant scale of turbulence, having eddy trains of strong, relatively constant size vortices which mask the output of the other eddy scales. These "pure tones" are to be found only under special flow conditions, usually at relatively low Rey. numbers.

Various workers $23,24,25,27,31$ have delved into theoretical and experimental determination of the spectrum of isotropic turbulence. Lighthill was not as concerned with this as developing a general expression for the total acoustic power output of the quadrupole field mentioned earlier. In fact, reasonable theories on this spectrum were published as early as the 1930's. Various findings are briefly shown in Figs. 1.5(a) (e);2.4(d) and discussed in Art. 1.2. They all point to a relatively low frequency spectral distribution. This has been further verified by Mawardi and Dyer's 36 supersonic wind tunnel noise studies (ref.Fig. $5: 1,9$ ).

An anomaly would seem to exist in view of what lighthill said on p.75/eg. The lower the wavelength of sound consi- 
dered, compared with the scale of the forcing function, the less the sound radiated.) He claimed the amplitude of the quadrupole field strength per unit volume is $\alpha$ square of a typical velocity $U$ in the flow; but that the amplitude of the radiation field due to a quadrupole $\alpha$ its strength $X(f r e q \cdot)^{2}$; and finally that in many cases a typical frequency $\approx U$. Centrifugal fans have a reputation for relatively low speed operation and low frequency noise spectrum. Air moving at low Mach numbers also has a low frequency noise spectrum. As blade tip speeds increase and flow velocity increases we can expect both the overall intensity and frequency of maximum intensity component or band to increase. If allowed to crudely illustrate this, we could pass from the low frequency, low intensity noise of a centrifugal fan coupled to a ventilating system, to the higher intensity, higher distribution of a supersonic wind tunnel powered by multiple stage high speed axial fans. Or, compare the low toned whisper from a low speed jet to the ear tingling high pitched whine of the jet aircraft "warming up". Since most noise measurements have been made on flows of low Mach numbers, it is simply lack of experimental evidence that creates this seeming anomaly. For a given type of forcing function, or scale of turbulence, spectral densities plotted against a scale of $\mathrm{f} / \mathrm{fmax}$ should lead to similar spectra shapes. 


\subsubsection{BOUNDARY TURBU IENCE AND AERODYNAMIC NOISE}

Air motion is usually generated artificially by blades and conducted by ducts, whether it be a ventilating system or supersonic wind tunnel. Turbulence is therefore created by the solid boundaries of the blades, and acted upon by the duct boundaries. The effect of boundaries according to Curle 34 is to cause reflection, diffraction, confine the quadrupole field, and to exert equal and opposite forces on the turbulent medium at the boundary. The resultant sound field generated by an isotropic turbulent field surrounded by boundaries consists of the sum of a doubly - differentiated volume integral solution (Lighthill's contribution) plus a surface integral over the solid boundaries (Curle's contribution). For similar flows, the sound intensities vary as $U^{8}$ and $U^{6}$ respectively ( $U$ being a typical flow velocity). The quadrupole field is notoriously weak (aerodynamic sound production efficiency $\eta \alpha \mathrm{Mach}^{5}$ ) at low Mach numbers. The dipole field over the boundaries is therefore the one of importance for flows of low Mach numbers $\left(\eta \propto \operatorname{Mach}^{3}\right)$. It is obvious, boundary or no boundary, that the efficiency of aerodynamic sound production is extremely low.

From this we would expect one blade of a centrifugal fan rotating with a tip Mach number on the order of 0.1 to emit a relatively wide noise spectrum, the overall intensity of which increases as the sixth power of the air velocity leaving the blade. There is evidence to show that this index may vary from about 4 to 7, for a number of mutually interfering blades and about 5 for noise generated by turbulent flow at moderate Mach numbers over thin stiff plates (eg.duct walls, fan housing). 
Gerrard (see Curle 34 ) investigated the sound from flow past a cylinder and found sound intensity $I \approx U^{7}$. He then varied the cylinder diameter and velocity in such a way as to keep Rey. constant and found a dependence on U 4. It is also suggested qualitatively that rotating parallel rods in their plane results in more noise and a higher power of $I \propto U^{X}$ than when they are rotated such that one follows in the wake of another. The theoretical study of noise radiated by the vibration of thin, stiff, flate plates under the action of turbulent boundary fluctuations, made by Kraichnan 44 shows a dependence on Mach 5. This is for Mach numbers on the order of .05 to .5, therefore includes higher ventilating air speeds. At lower Mach numbers, there is a tendency for this dependence to be flatter. Although more fundamental observations like those bove are necessary for proof; it appears quite likely that the rotating system represented by a fan, either centrifugal or axial, could result in "blade" turbulance plus "duct" turbulence (eg. both boundary layer turbulence) noise which depends on $U^{4 t 07}$ and is governed by details of blade profile and size, speed, length, Rey. number of the flow, flow geometry and environment. This is borne out by the results in Art.5.

What of the frequency spectrum of noise generated by boundary turbulence? Evidence is strongly in support of a very wide band spectrum, having most power at the lower end and decreasing continually with increasing frequency when the Mack numbers involved are say $<0.5 \mathrm{Mach}$. Curle 34 shows that if $Q$ represents the spectrum of the quadrupole field, and $D$ that of the dipole field, then $Q=2 D$ frequency-wise. It is known 
that the sound generated by the fluctuating forces at the solid boundaries will have the same frequency as the fluctuating velocity field, hence $Q$ contributes more to higher frequencies than D. What was said of the spectra for $Q$ in Art. 2.2.2 may be applied to $D$ provided the abscissa scale is halved. A study of wall pressure fluctuations in a turbulent boundary layer conducted by Willmarth 45 yielded a power spectrum of wall pressure fluctuations having a maximum at the lowest frequencies ( 300 c.p.s. was the minimum transducer response). The spectrum descended at decreasing rate with frequency and was continuous up to the maximum transducer response at $50 \mathrm{Kc}$. This result is for Mach $=.197$ in a $4^{\prime \prime}$ dia., $1 / 8^{\prime \prime}$ thick brass tube. Wall pressure fluctuations were found to be a minimum of 6 times greater with turbulent flow than with laminar flow, both being at the same Rey. number. In Kraichnan's 44 analytical study, mean - square radiated sound power for a Mach flow of .5 and .25 over stiff, thin, flat, low damped plates yielded spectra of the following relative values:

$\begin{array}{lcc} & \text { TABLE 2.2 } & \\ & \text { (after Kraichnan, Fig.6, ref.44) } \\ \text { c.p.s. } & \frac{M=0.25}{|\bar{p}(f)|^{2}} & \frac{M=0.5}{|\bar{p}(f)|^{2}} \\ 100 & \text { relative db } & -9 \\ 300 & -12 & -5 \\ 1000 & -9 & -1 \\ 3000 & -9 & 0 \\ 10,000 & -17 & -10 \\ \text { Max. }=-8.5 \text { at } 500 \mathrm{c.p} . \mathrm{s} . & 0 \text { at } 2500 \mathrm{c.p} . \mathrm{s} .\end{array}$


For Mach $\varlimsup 0.1$, it can be seen that there will be considerable low frequency power in the spectra. Again it is pointed out that this noise is mainly due to plate vibration induced by turbulent pressure fluctuations, hence represents a rather random field of pressure due to turbulence, as modified by plate parameters such as mass, size, stiffness and damping factors.

\section{A third finding of these two scientists concerns} pressure. Willmarth noted that the output power spectrum was roughly proportional to the dynamic pressure squared. In applying his theoretical results to aircraft, Kraichnan found that for critical damping of the plates the noise power $\propto$ $\rho^{2} P_{e x t}^{2}$ for the pressurized cabin, and $\approx P_{\text {ext }}^{2}$ for the unpressurized cabin, where $\rho_{0}$ is the sea level value of the air density and $\rho_{\text {ext. }}$ is that in the cabin. Over and under damping may considerably complicate the picture.

2.2.4 THE DUCTED CENTRIFUGAL FAN AS A NOISE SOURCE

The major source of noise in a well designed and installed fan is due to turbulence generated by the blades. This is what would be expected from the above considerations and is shown to be true in the results. The results also indicate this is highly dependent on operating conditions.

Let us generalize here and list all the possible noises generated due to the system of Fig.1.3. The fan runner is assumed to have an element of dynamic unbalance, as is always the case in practice. 
PRINCIPAL NOISE GENERATORS

(in approximate order of importance)

1. Wideband noise due to turbulence at the blades.

2. Rotational line component and possible harminics due to:

(i) Fan runner eccentricity "pumping" air in pulses down the duct at frequency r.p.m./60. This is illustrated in Fig. $2.4(\mathrm{e})$.

(ii) Vibration of the scroll case set up by dynamic runner unbalance. Due to the finite structural flexibility of the welded steel plate construction usually used, the back part of the scroll case will cause positive displacement of air in the form of pulses propagating down the cuct. This will be greater or less and may or may not have harmonics, depending on the rigidity of fan housing and mounting. This action is illustrated by Fig. $2.4(\mathrm{e})$ and is somewhat analogous to a loudspeaker diaphram forcing the mass in a fixed region of space to fluctuate.

3. Line component and possible harmonics due to air pulses resulting from blade spacing. This is amplified somewhat by the fan "cut-off" (see Fig.2.4(e)). The closer this is to the blades, the greater this component due to larger changes of air acceleration involved locally about the "cut-off".

4. Wideband noise due to air turbulence acted on by the duct walls. This is greater or less, depending on the Mach number of the flow.

5. Mechanical vibration (aside from the "pumping" action mentioned in point 2) of the steel plates of which fan 
and ductwork are composed, bearing noise and all such "mechanical origins".

\section{MICROPHONE AND WINDSCREEN}

An additional measured "noise" is due to pressure pulses generated by and at the microphone when it is located in a fast moving air stream. The subject of windscreens was studied recently at McGill University (ref.Bredahl6). Local pressure pulses generated by the moving air over a microphone can cause output voltages well exceeding that from the real noise propagating in the airstream. This is minimized by a screen which will present a high impedance to airflow, a low impedance to sound. The screen employed (see Art.3) was found to be one of the best tested by Mr. Bredahl. However, as he concluded, the complete elimination of windnoise appears impossible because:

(i) Even though the moving air is retarded in steps to reduce the decceleration of the motion, and though low air motion impedance occurs in the region surrounding the screen while the screen is a high impedance, rapid decceleration of some of the air is unavoidable. This causes changes in the rates of momentum flux, resulting in a finite component of aerodynamic noise.

(ii) The screen causes additional turbulence in the airflow with resultant increase in noise.

(iii) Vibration of the microphone in the airstream adds to the voltage output.

(iv) Bombardment of the screen by air molecules. These are negligible or significant, depending on screen design, 
materials, flow conditions and support of the microphone. This will be discussed further in Art. 5 .

There are other minor noise sources in any ventilating system, but they are either of no consequence compared to the above, or can be made so by proper design.

\subsection{HYPOTHETICAL VEN TILATING SYSTEM NOISE EXAMPLE}

No pretense is made at covering aspects of ventilating system noise other than that from ducted fans. However it is instructive to examine a very simple ventilating system merely to assess the meaning of fan noise in terms of speech interference and annoyance.

The basic assumptions are represented by Fig. 2.5 and curve $I$ of Fig. 2.6. A continuous noise spectrum is assumed for the centrifugal fan. Guidance for the ventilating system was obtained from ref.52, while Beranek ${ }^{57}(\mathrm{ch} .4,10,12)$ was employed for those curves followed by his name in the legends of Fig. 2.6, 2.7 and also for calculation procedure and noise criterea. Fig. 2.5,2.6, 2.7 are largely self-explanatory.

The end result is curve 1 and 3, Fig. 2.7, which shows the relative SPL at the ear of a listener positioned as in Fig. 2.5(b). This is the worst listening position in the hall under the assumption that the air outlet behaves like a piston in an infinite baffle. This assumes the air molecules are all vibrating in phase over this 25 square foot area, which is far from true. This assumption gives higher directivity factors 
Fig. 2.5

$p \circ g=86$

Illustration for Hypothetical Fan Noise Example
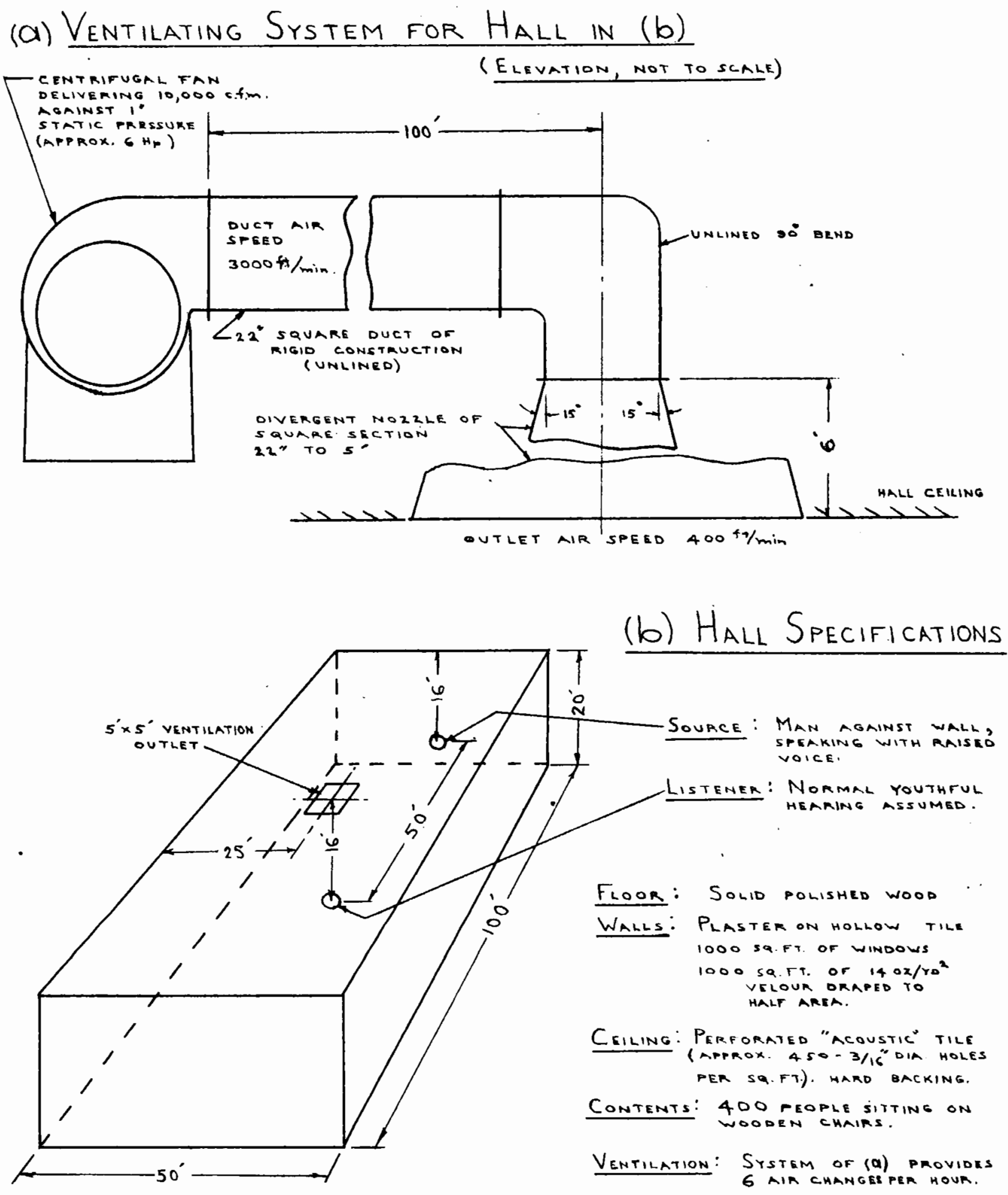
Fig. 2.6

Noise Spectra of Ventilating System OF F $16.2 .5(a)$

in

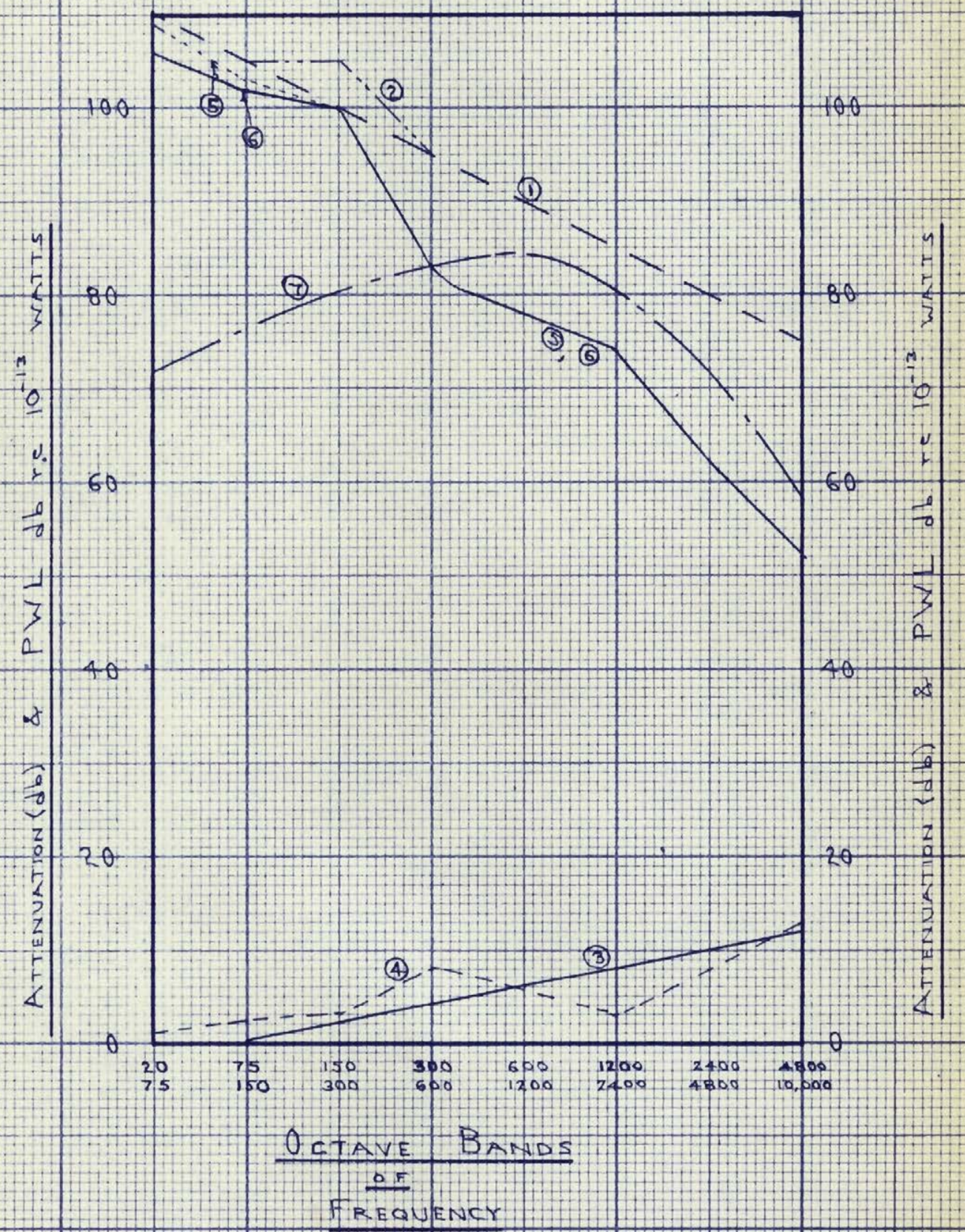

LEGEND:

CURVE D) AsSUMED FAN SPECTRUM

(3) AdDTtional component dUE to AIR furbulamé WHHIN the

(3) Assumed at fenuation of 100 et main DUCT, file. 2.5 (a)

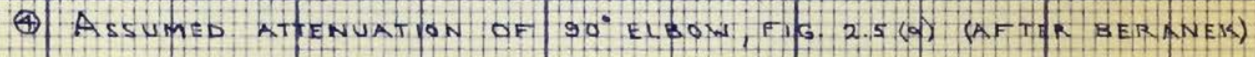

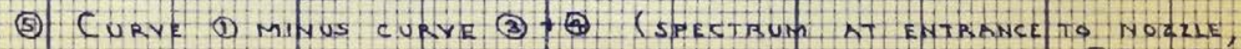

(8) PWL OF VENTILATING SYSTEM AT DIF TUSER MOUTH

(8) PWL OF AYERAGE MAN'S RAISED VOICE (ATAER BERANK) 
SPECTRA AT ListeNER in HaLL OF

$$
\text { Fig: } 2.5(b)
$$

EGEND:

CURVE Q SPL DUE TO VENTILATING SYSTEM

ASGUMING ATR OUTLET TO BEHAVE LIKE

A piston in an intrinite natele.

(2) LoUDNES or 0

(3) SPl of rased maks voleE At

$\left[\begin{array}{c|c}\text { Curves } & (3) \text { to } \phi) \\ \text { Nhter } & \text { DERAMUER }\end{array}\right]$

(9) PeAK or 3

(5) Anerage man's vonce in formal conyerishtion

(6) Minimam levers or (3)

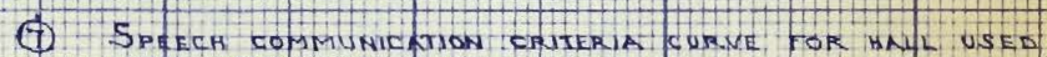

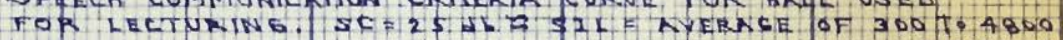

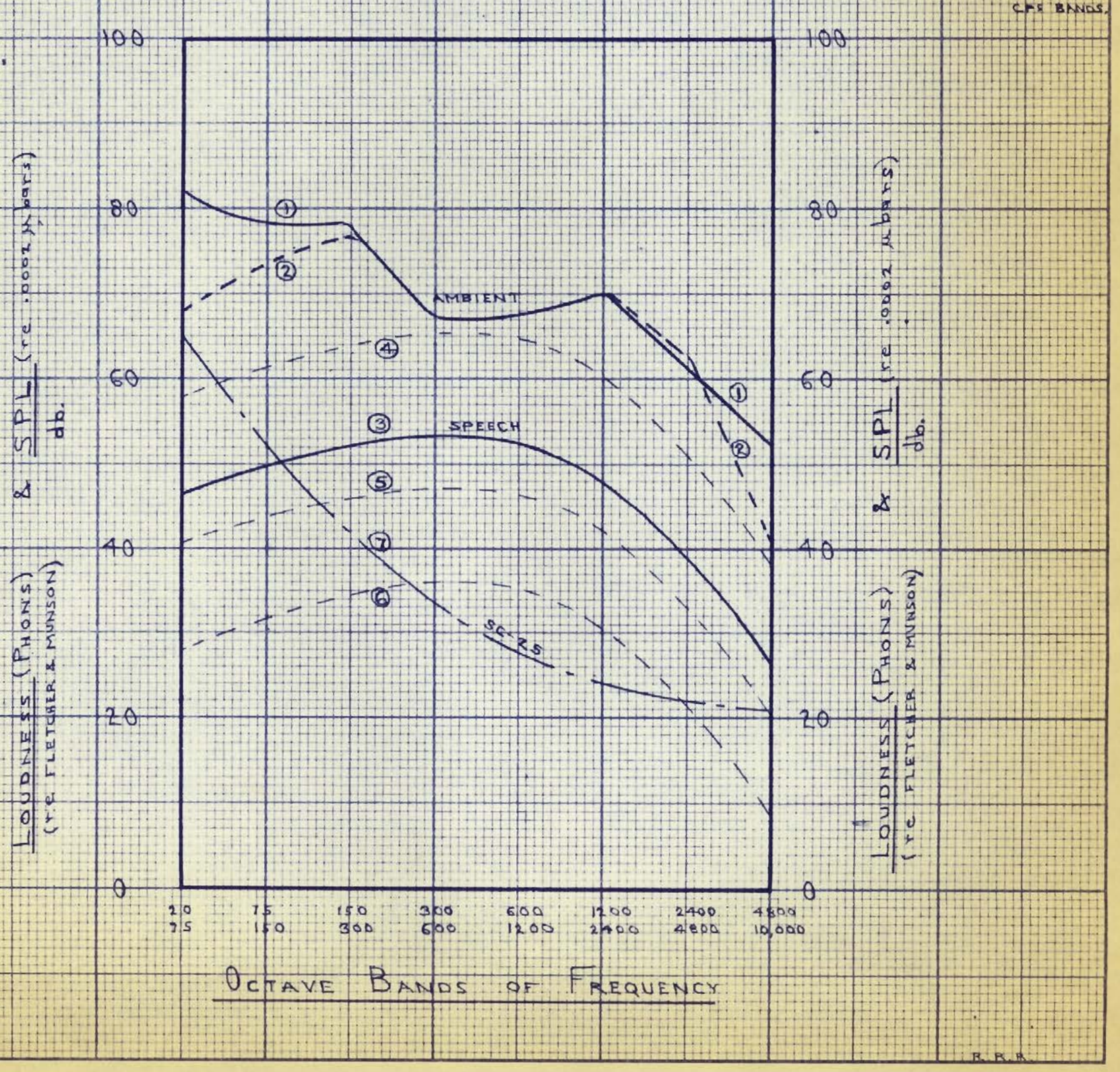


at the higher frequencies than would normally be the case. The true picture would be a curve commencing as curve 1 Fig. 2.7 does, but declining at a much faster rate above about 300 c.p.s. Since the fan noise completely covers the region between curves 4 and 6, Fig. 1.7, the Articulation Index (Beranek p.409) is zero, and the speaker (Fig.2.5(b) ) cannot be heard.

If the hall were used chiefly for lecturing, either a public address system must be employed, or fan noise lowered so that most of the area bounded by curves 4 and 6 Fig. 2.7 and frequency bands $300 \mathrm{c} . p . s$. to $4800 \mathrm{c} . \mathrm{p} . \mathrm{s}$. lies above the ambient or fan noise curve at this worst location. (Actually the back of the hall would be the poorest listening area if the simplifying assumption that the ventilation noise is generated by a piston source was discarded. In the interests of brevity, the point directly beneath the air outlet is assumed the poorest listening area because of the high directivity at the high frequencies of the air outlet source.) Unless noise in the frequency bands 20 to $300 \mathrm{c.p} . \mathrm{s}$. and $4800 \mathrm{c.p} . \mathrm{s}$. upward is relatively excessive, it will not serve to mask the important 300 to 4800 c.p.s. speech spectrum band too badly.

If the hall is also used for chamber music and concerts, it is highly desirable that fan noise be reduced to a level below the minimum desired SPL spectrum of music. In this case the entire audio spectrum will be involved.

Assuming flat electronic amplification is used in the hall (due to the nature of the transducers now in use, flat amplification is rarely approached without high investment), curves 3 to 6 Fig. 2.7 can be raised an amount equal to the db 
gain of the system, with due regard for directivity. This method of overshadowing ambient noise can be applied only as far as listening fatigue will permit. It is much more desirable to reduce the noise level, thereby reducing the overall SPL in the hall necessary for good understanding of spoken word or music. With this in mind, Beranek gives a noise figure of $\mathrm{SC}=25$, applicable to this hall. SC stands for speech communication, and the number is the speech interference level (SI L) for that contour (SI L = arithmetic average SP L of noise in bands $600 / 1200,1200 / 2400,2400 / 4800$ c.p.s. If noise in the $300 / 600$ band $>10 \mathrm{db}$ more than in the $600 / 1200$ band, it is also included in the average). The recommended noise curve for this hall if used primarily for lecturing is curve 7, Fig. 2.7. The required ventilating noise attenuation is given by curve 1 minus curve 7, Fig. 2.7. The noise criteria curve NC $=25$ (ref. Beranek 47 ) is the same as curve 7, Fig. 2.7 above 600 c.p.s., but is lower below this frequency, being $57 \mathrm{db}$ for the 20/75 c.p.s. band. If annoyance, as well as speech communication is a factor, the NC $=25$ curve should govern.

In these calculations, the room constant $\left(R=\frac{5 \bar{\alpha}}{1-\bar{\alpha}}\right.$ where $S=$ surface area and $\bar{\alpha}=$ average absorption coefficient of the room), reverberation time $\left(T=.049 \frac{V}{a}, V=\right.$ volume of room in cubic ft. and a - absorption units in Sabines, sq.ft.), and the like were computed for 125,250, 500, 1000, 2000 and 4000 c.p.s. using Ch.10 Beranek, and extrapolated to include higher and lower frequencies.

Formula employed were: 
(a) Assuming an equivalent conical horn for the air outlet nozzle of Fig. 2.5(a), the throat acoustic impedance of an infinite conical horn is $r_{A}+j x_{A}$ :

$$
\begin{aligned}
& r_{A}=\frac{\rho_{0} c}{S_{0}} \frac{k^{2} x_{0}^{2}}{1+k^{2} x_{0}^{2}} \ldots \ldots \\
& x_{A}=\frac{\rho_{0} c}{S_{0}} \frac{k x_{0}}{1+k^{2} x_{0}^{2}} \ldots \ldots
\end{aligned}
$$

after Olson and Massa ${ }^{60}$.

$r_{A}+j x_{A}=$ throat acoustic impedance. $x_{0}=$ distance between apex of horn and actual throat.

So $=$ area of throat.

other symbols as per glossary.

(b) $\frac{p_{r}}{p_{i}}=\frac{z_{2}-z_{1}}{z_{2}+z_{1}}$ (see Eq. 2.8)

$T=1-\left|\frac{P_{r}}{P_{i}}\right|^{2}=$ transmission coefficient

$\underset{\text { Thans) }}{\operatorname{Transmission}}=10 \log _{10} \frac{1}{\mathrm{~T}} \mathrm{db}$

(c) The sound pressure level at a distance $r$ feet from a source having a power level PWL of directivity $Q$ in a large enclosure of room constant $R$ is:

$S P L=P W L+10 \log _{10}\left(\frac{Q}{4 \pi r^{2}}+\frac{4}{R}\right)+0.5 \mathrm{db}$

at standard temperature and pressure.

(Eq. 2.22 is a function of frequency).

(d) The directivity pattern for a rigid circular piston in an infinite baffle is given by

$$
\begin{aligned}
& {\left[\frac{2 J_{1}(k a \sin \theta)}{k a \sin \theta}\right]} \\
& J_{1}=\text { Bessel function of first kind and order } \\
& a=\text { piston radius } \\
& \theta=0^{\circ} \text { on piston axis }
\end{aligned}
$$

This, in conjunction with the concept of directivity factor: $Q(f)$ - ratio of the intensity on a designated 
axis of a sound radiator at a stated distance $r$, to the intensity that would be produced at the same position by a point source if it were radiating the same total acoustic power as the radiator; yields a curve of $n Q(f)$ vs $k a(\theta=0)$ p.112 Beranek. This curve was used for estimating the directivity factor, as a function of frequency, of the ventilating outlet treated as a piston source in an infinite baffle.

It was mentioned above that the desired attenuation to be built into the ventilating system of Fig. 2.5(a) is curve 1 minus curve 7. Above $300 \mathrm{c} . \mathrm{p} . \mathrm{s}$. this is readily achieved simply by lining the duct with about a one inch thickness of one of the commercial duct lining materials available today. For lower frequencies, more elaborate means of attenuation are usually required such as thicker absorbent lining, wideband filters of various sorts, and reduction of air speed. Concerning air speed, it does little good for example to attenuate fan noise well, only to have noise generated by air flow turbulence near the outlet propagated into the room. An improvement in the system of Fig.2.5(a) in light of the relatively high air speed in the main duct of $3000 \mathrm{ft} / \mathrm{min}$ would be to reduce this speed by employing a larger duct. This is not always possible for reasons of space and economy. What should be done is to gradually increase flow area prior to the elbow as well as after it. This will reduce the Rey. number of the flow, generally reducing air turbulence and noise at the elbow. The elbow itself will attenuate some of the noise generated by upstream turbulence, whereas as it now stands, some air turbulence 
noise is undoubtedly generated within the elbow and at the junction of the elbow and air outlet transition piece or nozzle.

There is negligible engineering information available so far giving insertion loss of lined duct sections, elbows, filters and other "plumbing" when they carry an air stream. The data now available simply gives attenuation under static conditions. There is evidence (ref.Lambert 48,49) that well intended noise attenuating devices, filters, traps, etc. may in some cases possess insertion gain, rather than loss, when air is flowing through them. This should not be too surprising to those acquainted with noise from a turbulent boundary layer. An element with fair static attenuation may well generate noise under dynamic application (eg. air flow) due to the turbulent scale it creates. This turbulence may generate noise compoments which could well be resonated by the element. Lambert points out that more refined techniques are necessary in order to evaluate the performance of acoustic devices in viscous moving media. Until such time as reliable data from further research is provided, acoustic filtering must be applied with discretion, keeping the above facts in mind. 


\section{APPARATUS}

The basic system for the acoustic waveguide technique of $f a n$ noise measurement was discussed in Art. 2 in connection with Fig.1.3, and is elaborated upon by its builder, G.Chipps?. Specifications of N.A.F.iv. bulletin No. 110 were followed as far as they went. The General Radio family of sound measuring equipment was used to measure and analyse the noise. A complete equipment list follows in Art. 3.1. Comments and data on equipment performance occur in Art. 3.2. A wide variety of photographs of the system are found in Ref.7. Supplementing these are additional displays of equipment, Fig.3.1, 3.2, 3.3.

\subsection{EQUIPMENT LIST AND DESCRIPTION}

\section{$\triangle$ MATCHED ACOUSTIC WAVEGUIDE AND NOISE SOURCE}

(Refer to Fig. 1.3)

1. Canadian Sirocco Series 81, No. 135, class I and II, single inlet, single width centrifugal fan.

Arrangement: No. 2, top horizontal discharge.

Runner: 36 forward curved blades approx. $7 \frac{1}{4}$ "wide $x 11 / 8$ " (Fig.3.1a)

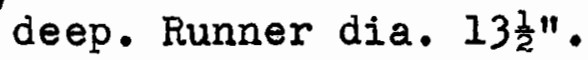

Inlet: 14 3/8n diameter outside, $1.088 \mathrm{sq.ft.} \mathrm{inside.}$ Outlet: $143 / 8 " \times 103 / 4$ " outside, 1.044 sq.ft. inside. Performance: Fig. 3.4 (a).

2. Canadian High - Efficiency Streamline (HS), Series 8l, No. 135, class I and II, single inlet, single width centrifugal fan.

Specifications as for I except:

Runner: 12 backward sloped blades approx. $41 / 16$ " wide $x$ (Fig.3.1b) 
FIG. 3.1

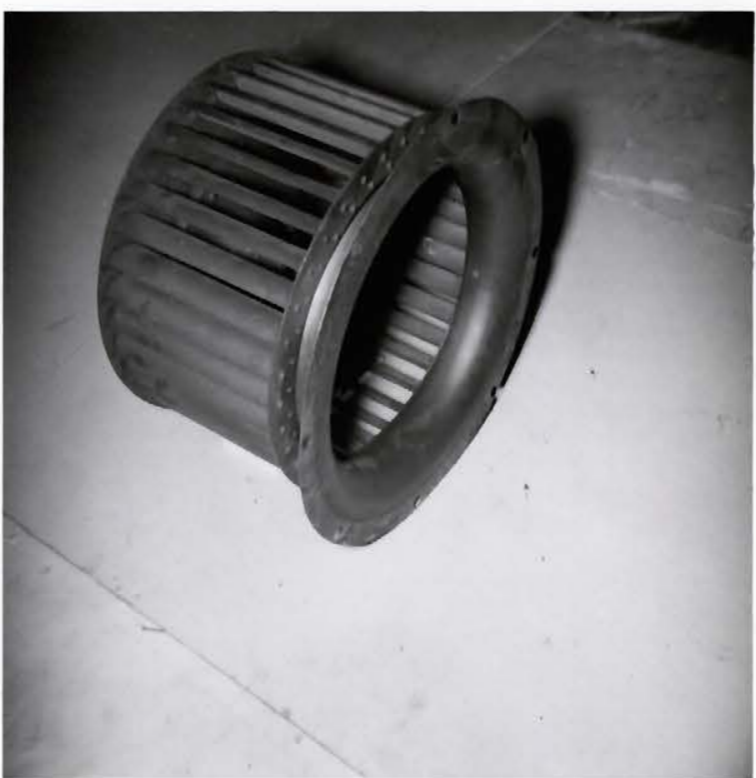

(a) SIROCCO RUNNER \& INLET FLANGE

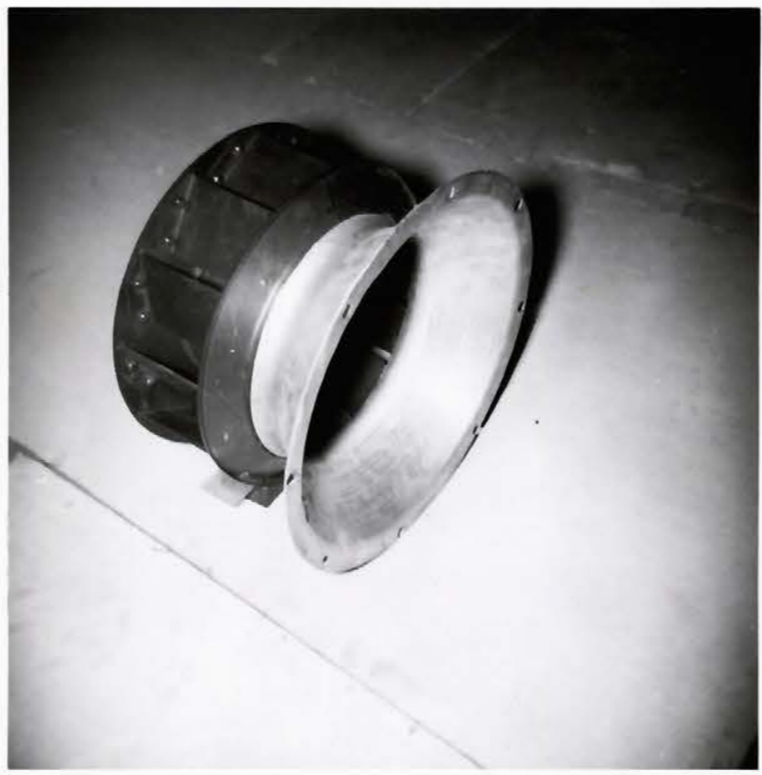

(b) "HS" RUNNER \& INLET FLANGE 
FIG. 3.2

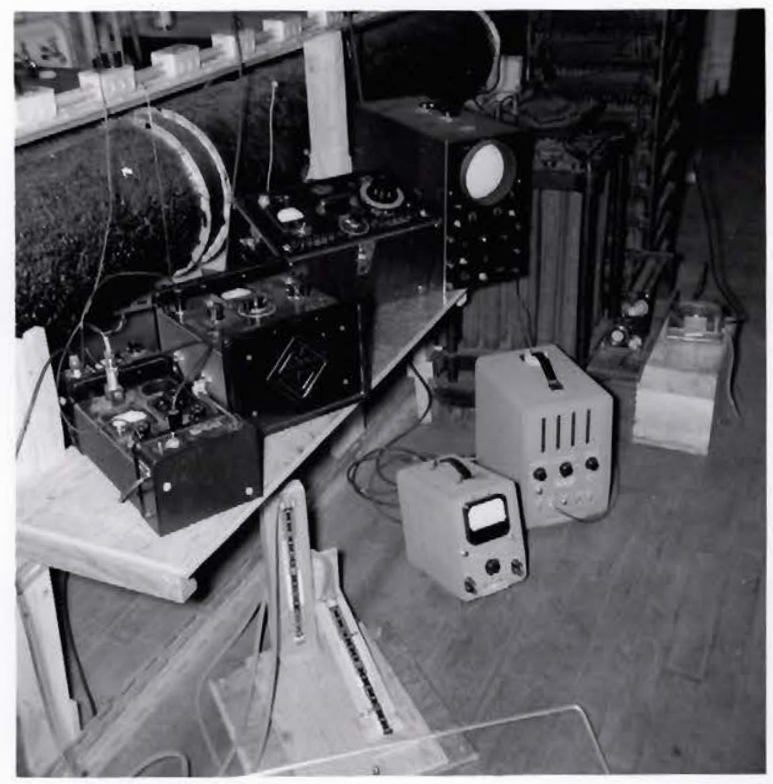

(a) DISPLAY OF INSTRUMENTS

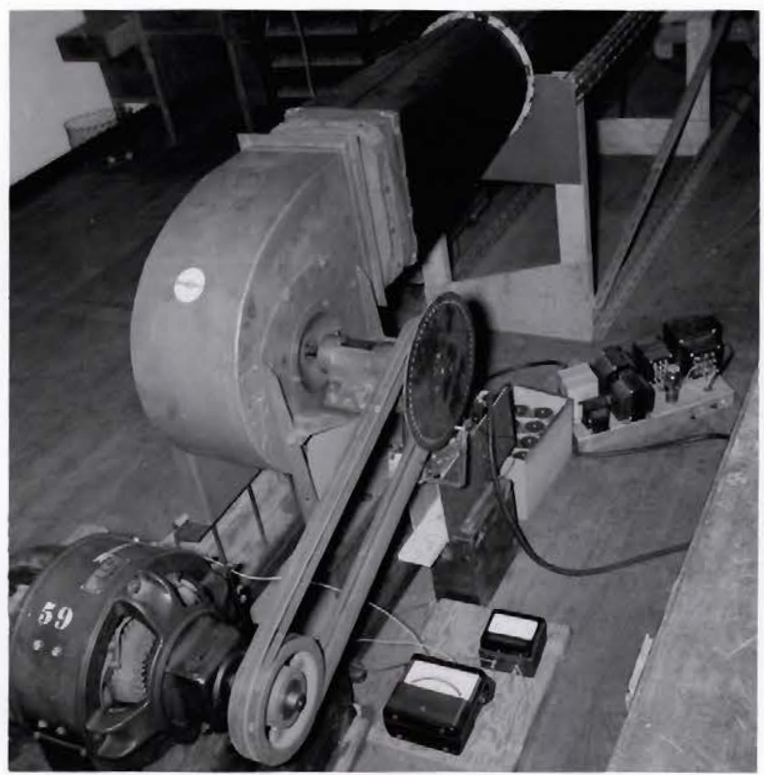

(b)

FAN, MOTOR \& SPEED

COUNTING EQUIPMENT 
FIG. 3.3

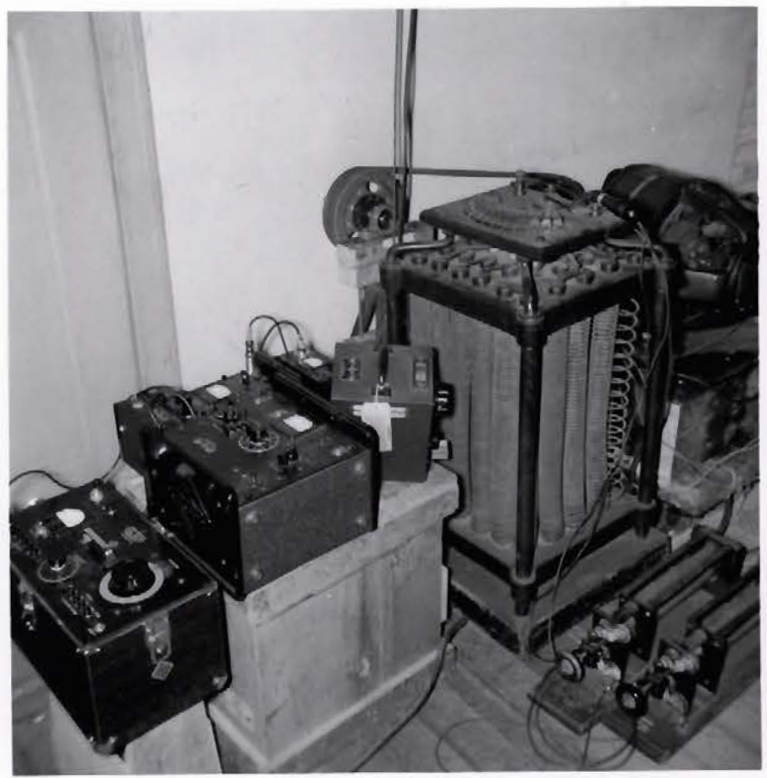

(a) MEASURING \& DRIVE EQUIPIENT EXTERIOR OF ANECHOIC CHAMBBER

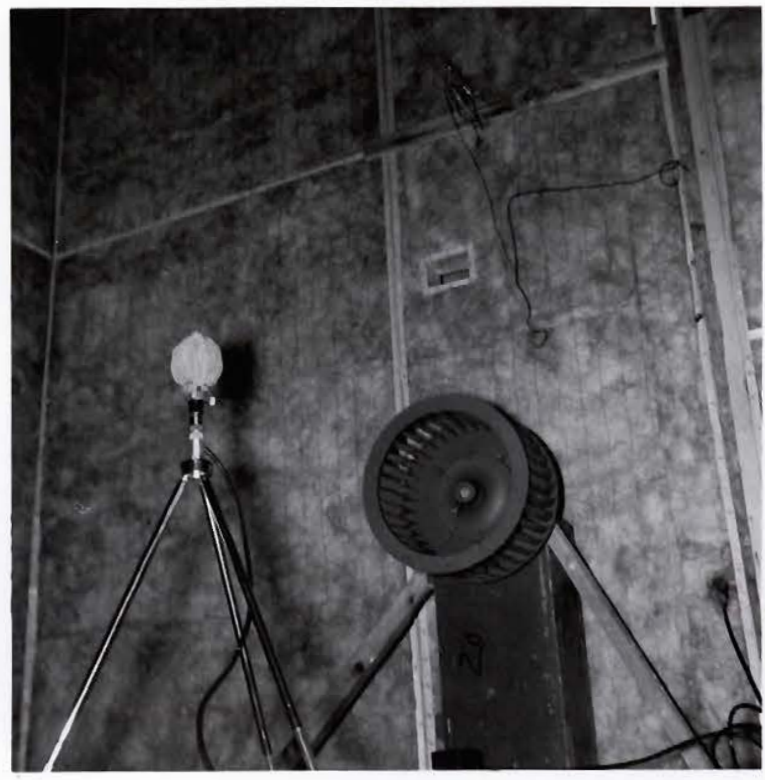

(b) INTERIOR OF ANECHOIC CHAMBER 


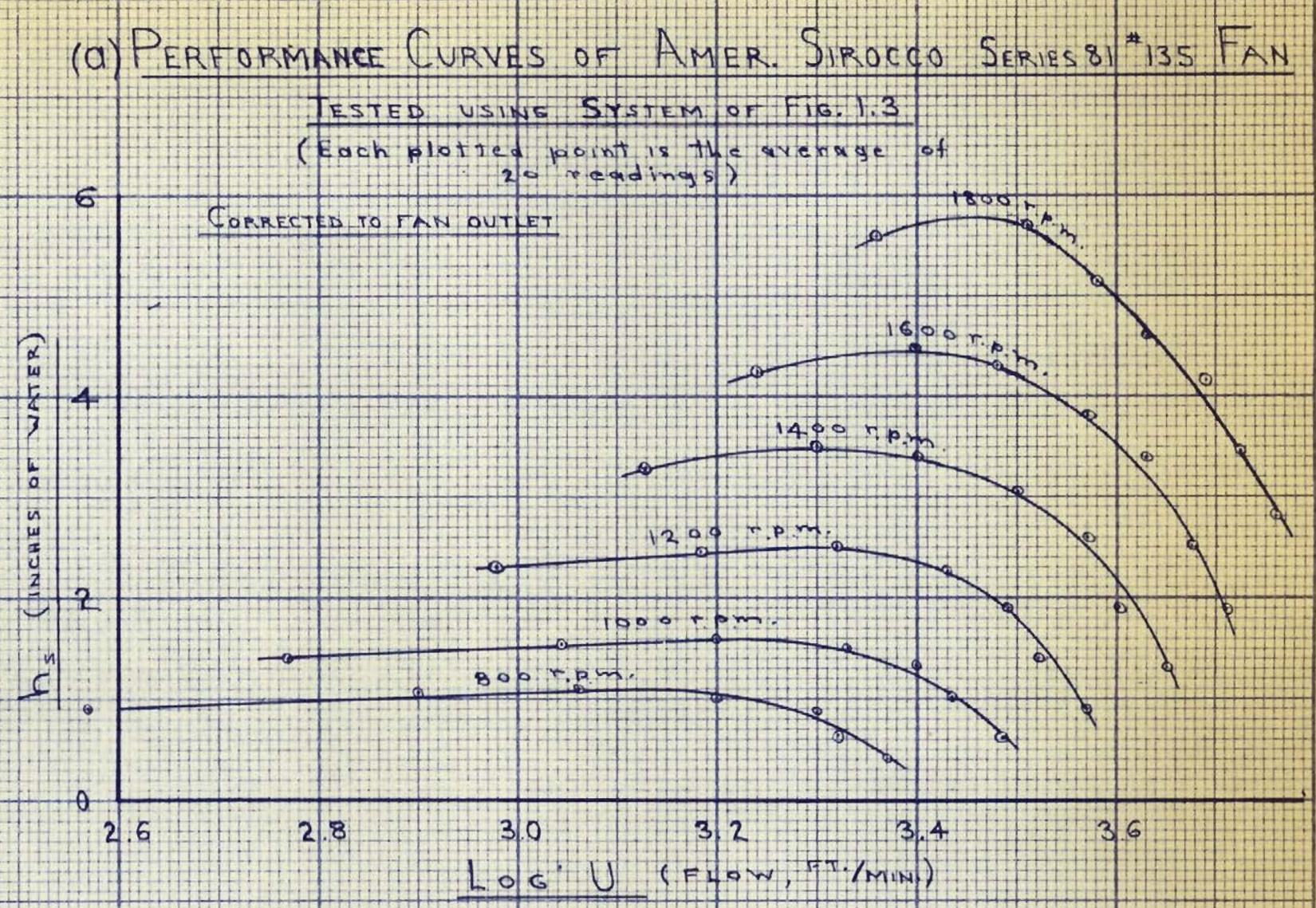

(b) Performance Curves of Amer. "HS Series bi"izs Fan

TESTED USING STSTEM of. FIG.1.3

(Each plotted point is the averade

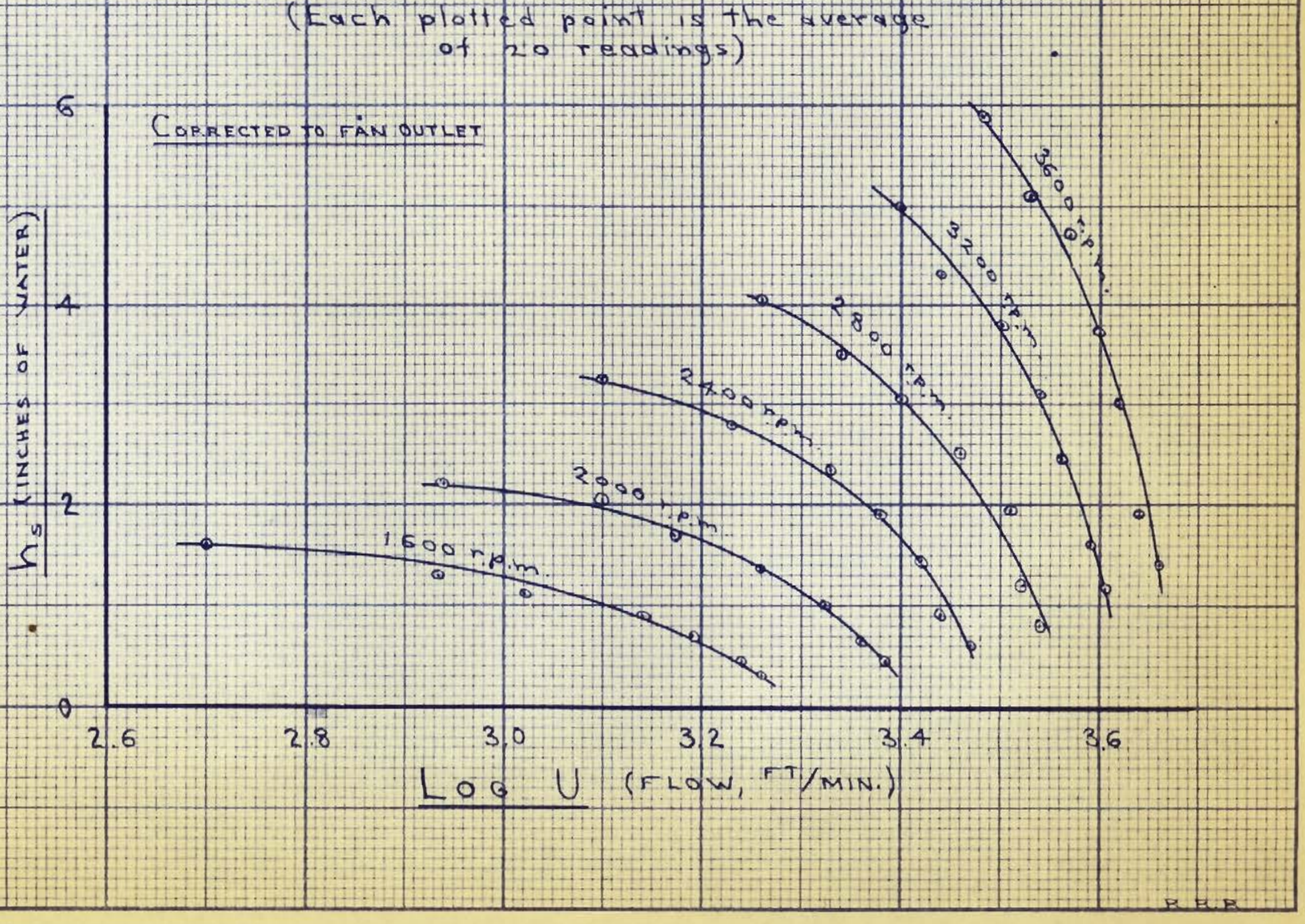




$$
\text { 3" deep. Runner dia. } 13 \frac{1}{2} \text {. }
$$

Performance: Fig. 3.4 (b).

NOTE: $I$ and 2 are identical to the respective American Blower Corp. fans.

3. A double canvas coupling 6" long, supported in the middle and each end.

4. A rectangular ( $1019 / 32^{\prime \prime} \times 149 / 32^{\prime \prime}$ inside) to circular (13 15/16" dia.) transition 29 3/4" long.

5. A circular section (13 15/16" dia.) 80 1/8" long.

6. An egg - crate air straightener $33 / 4$ " long with 9 vanes by 9 vanes on $13 / 8 "$ centers (inside dia. $137 / 8 n$ ). The vanes are about 1/16" thick. Intersection points are rigidly soldered.

7. A circular section $561 / 8 "$ long which is the slotted measuring section.

8. A circular to rectangular transition $301 / 4 "$ long. Dimensions as in 4.

Items 4 to 8 are fabricated from \#24 ga. U.S.S. galvanized iron. It is smooth inside, and coated partly with approx. 3/8" combination of water soluble tar and cork and partly with automobile undercoat on the outside. Item 7 has a l" wide $x$ 30" slot bounded on the sides by guide strips brazed to the duct. A heavy weight - loaded copper slider lined with hard rubber on the downward face was used for longitudinal movement of the microphone. A threaded stem passing through a rubber lined hole in the slider allowed vertical movement of the microphone within the duct over a $6^{\prime \prime}$ range with windscreen, 8" without windscreen. A $5^{n} \times 5^{\prime \prime}$ contoured, hinged, gasketed door was placed over a window in the side of item 7 to allow 
for checking of microphone position.

9. Modified exponential horn transition $553 / 8$ " long from Item 8 to Item 10.

10. An anechoic chamber 43" long and $421 / 2 "$ by $421 / 2 "$ cross section. Sixteen fiberglas wedges are suspended in the chamber.

11. An extension to Item 10 with movable panel to adjust system friction (i.e. back pressure loading on fan).

12. A 10" Iong removable measuring section was added by the writer as shown in Fig. 3.5 between items 9 and 10 .

See Fig. 3.5 for items 9 to 12 .

Items 9 to 12 were formed from $1 / 2 "$ and $1 / 4 "$ plywood. Where 1/4" plywood was used, it was rigidly braced. The horn was coated with damping material as used for the duct. Items 9 to 12 were all lined with 1 inch thickness of PF-612, $2 \frac{1}{2} \mathrm{lbs} / \mathrm{cuft}$ fiberglas. The wedges, made of the same grade of fiberglas and spaced as shown in Fig. 3.5 were designed from data by Beranek and Sleeper 50. They are 35" long with an 8 inch square base and a base length of 6 inches. The horn was designed for a $70 \mathrm{c.p.s}$. cut-off, the wedges for roughly 90 c.p.s. Variable system friction was simulated by varying the outlet area at the end of Item 11 by means of two panels each containing 63 - 2" dia. holes. By sliding one panel relative to the other then bracing and locking it in position, the area could be varied from approximately $3.8 \mathrm{sq} . f^{t}$. to zero, although there was leakage around these panels which increased the effective area. 
Anechoic Termination and Instrument Assembly AT HORN MOUTH REF. FIG: $1.3 p .18$

SCALE: $1 / 2^{\prime \prime}=1^{\prime}$

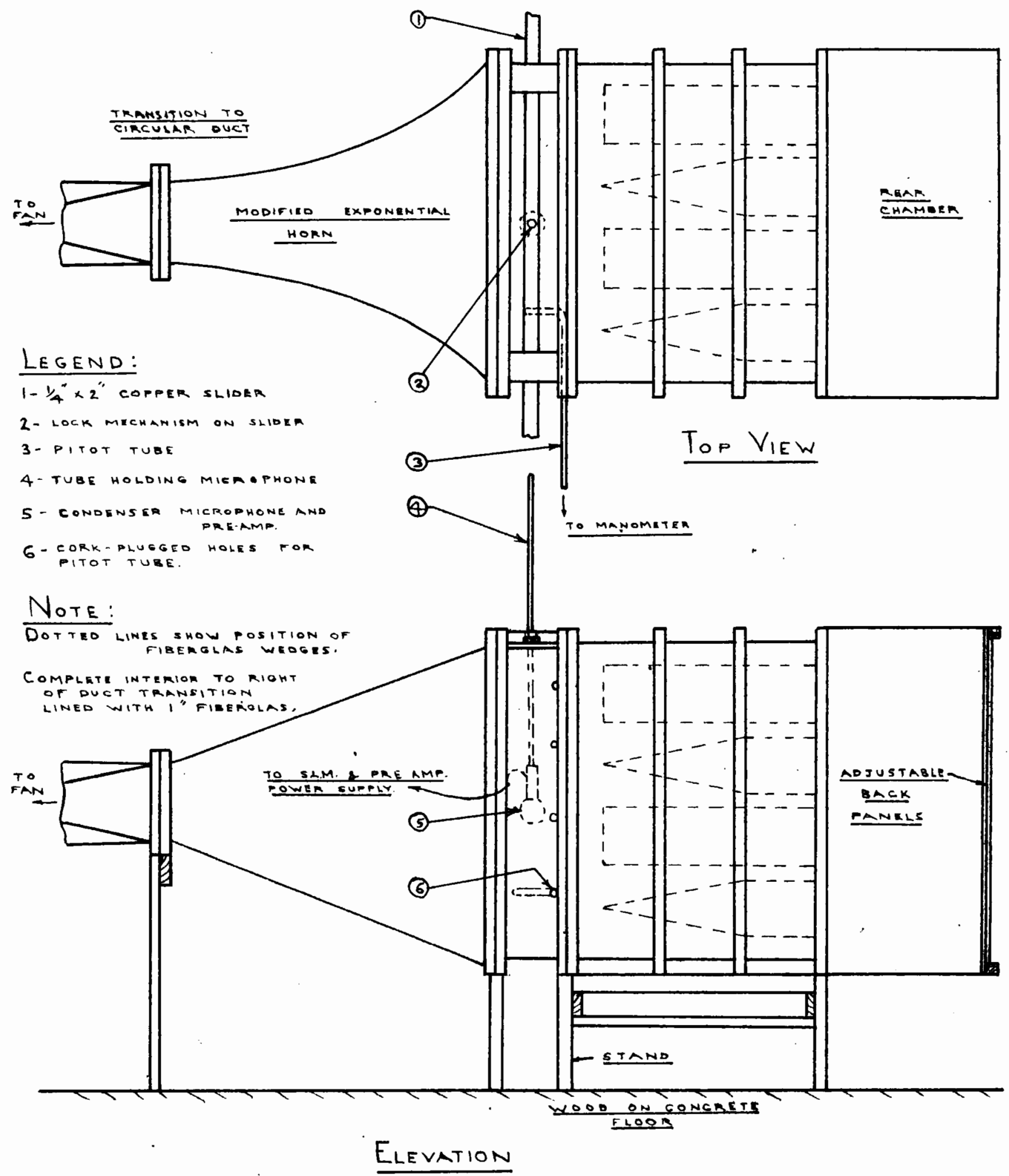




\section{B. POWER AND CONTROL EQUIPMENT}

1. Western Electric \#28 A755 shunt wound 50A, $1800 \mathrm{rpm}, 115 \mathrm{~V}$, $5 \mathrm{Hp}$ open frame d.c. motor.

2. Triple "B" V-belt drive.

3. Weston model $9010-3,0-150,0-300$ d.c. voltmeter.

4. Simpson model 29 0-1.5 A.d.c. ammeter (field).

5. Weston model 1147 0-100 amp d.c. ammeter.

6. Three rheostats for armature speed control; ribbon bank and coil spring bank for course control, carbon pile for fine control.

7. Speed counter circuit as follows:

(a) Disc with 60 evenly spaced holes drilled around the periphery, fastened to fan rotor shaft.

(b) Photo - electric pick-up and 3-stage amplifier.

(c) Multiple voltage power supply for (b).

(b) and (c) are war surplus equipment.

(d) Electronic counter, Hewlett Packard model 521 d.

This circuit provided accurate and instantaneous fan

rotor speeds displayed visually at the measurement location.

It was initially assembled by $A$. Bredahl ${ }^{6}$ for an allied project.

9. General Radio (GR) type \#63I BI Strobotac for speed checking purposes and for speed measurement at the anechoic chamber.

10. Hasler-Swiss precision dial tachometer for cross checking fan rotor speed.

C. NOISE MEASURING INSTRUMENTS AND RELATED APPARATUS (General Radio manufacture unless otherwise specified)

1. Type \#1551 - A Sound Level Meter, ser.\#244.

2. Type \#1262 - A Power supply for Item 1, ser.\#266

3. Type \#1551 - PI Condenser microphone power supply, ser.\#277

4. Type \#1551 - PI - 25, 640 - AA preamplifier ser.\#194

5. Type \#640 - AA condenser microphone, ser.\#5140 
6. Type \#760 - B Sound analyser, ser.\#1303

7. Type \#1550 - A Octave band analyser, ser.\#332

8. Type \#1552 - B Sound level meter calibrator, ser.\#1028

9. Type \#759 - P35 Vibration Pickup

10. Type \#759 - P36 Control Box (for item 9)

11. Dessicator for storing item 5

12. Iu Mont model 208 - B Cathode ray oscilloscope

13. Hewlett - Packard model 200 - B audio oscillator (used in conjunction with item 8)

14. Hewlett - Parkcard model $400 \mathrm{D}$ vacuum tube voltemeter

15. Power audio oscillator (Hewlett - Packard model 200 1) and Williamson amplifier housed in R.C.A.F. serial 1166 frame

16. General Electric S 1249 D 12 inch loudspeaker; response to 35 c.p.s.

17. Triple spherical wingscreen ( $23 / 4^{\prime \prime}$ diameter) designed and tested by $A$. Bredahl6. It consists of three wire mesh screens, one within the other, covered with tricot nylon cloth. The screen clamps rigidly to the microphone preamplifier so that the microphone is centered within the inner sphere. The attenuation of this screen was measured in an anechoic chamber by A. Bredahl, the results appearing in Fig.3.7. Further tests conducted by the writer are presented in Art. 5.

\section{MISCELLANEOUS}

1. Fan base.

The base used throughout the project was the solid steel one in Fig. $3.6 \mathrm{a}$, bolted to the floor ( 2 inches of wood on concrete). Two other bases tested were a heavy wooden one not fastened to the floor, and this same one mounted on vibration isolators. The method of vibration isolation is shown by the felt pads mounted on $2 \times 4^{\prime} \mathrm{s}$ in Fig. 3.6b. The pads were evenly loaded at $11 \mathrm{lbs} / \mathrm{sq}$.in. Natural resonance of the base was about 4 c.p.s. Negligible vibration was transmitted to 
(a) Photograph of Fan Showing Steel Base Used FOR THE RESUlts \& also Sample Vibration IsOlators USED ON EXPERIMENTAL PLATFORM (b)

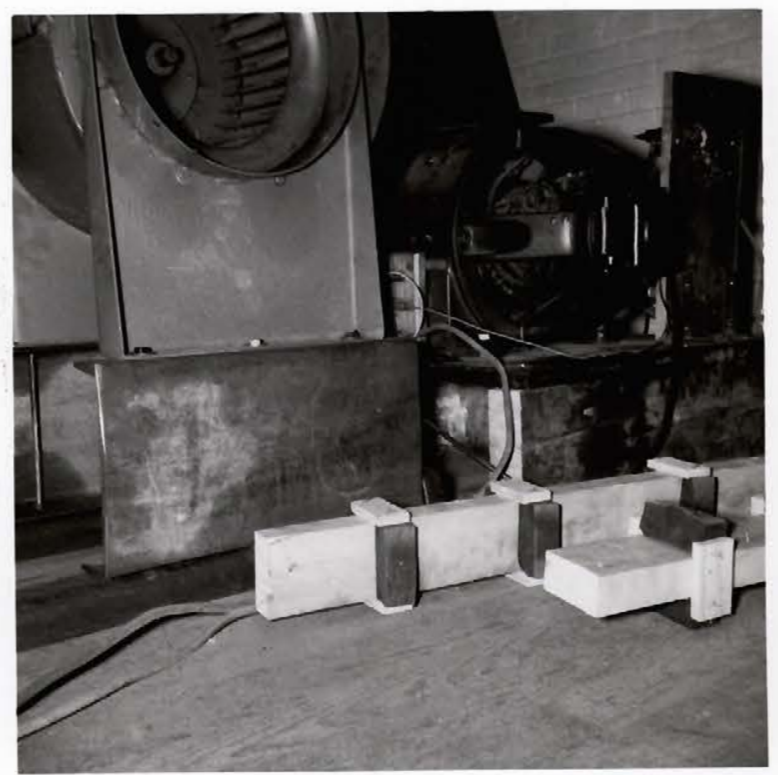

(b) Details of Fan \& MOTOR Platform Vibration IsOlation
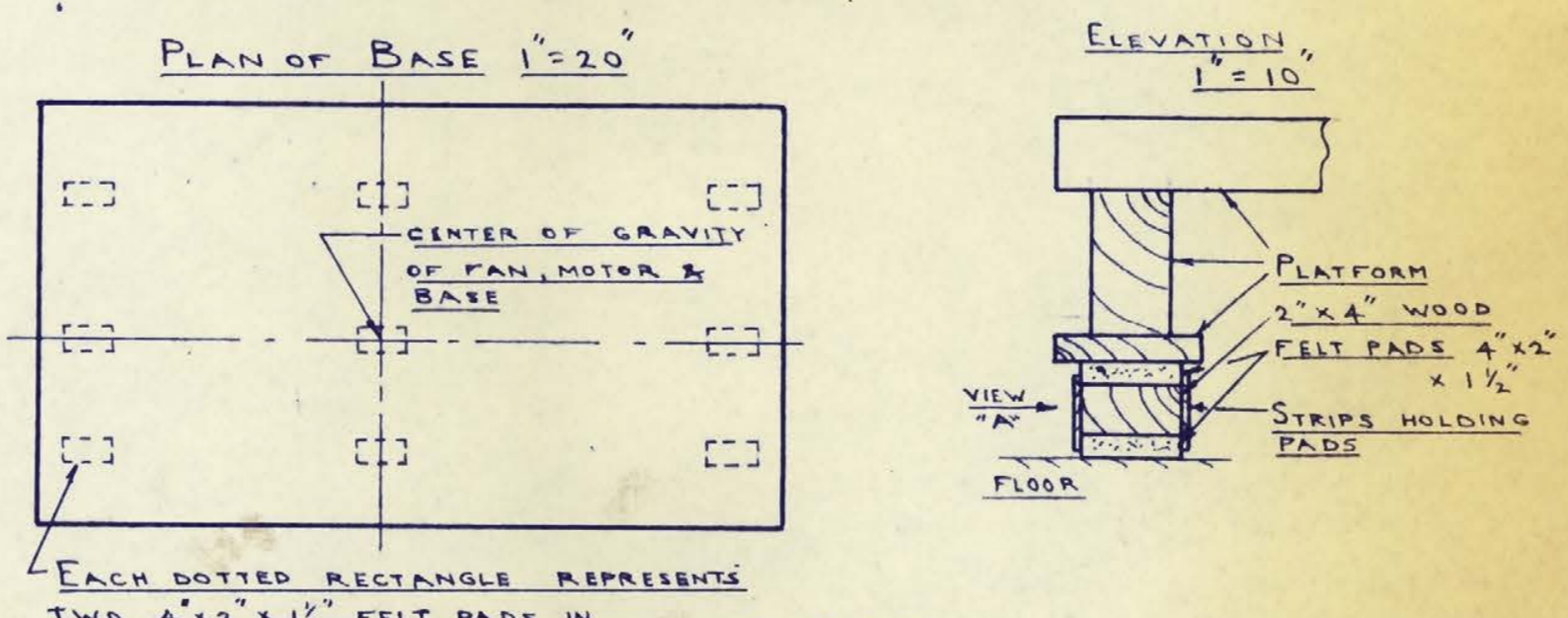
IWO $4^{\circ} \times 2^{\prime \prime} \times 11^{\prime \prime}$ FELT PADS IN SERIES.

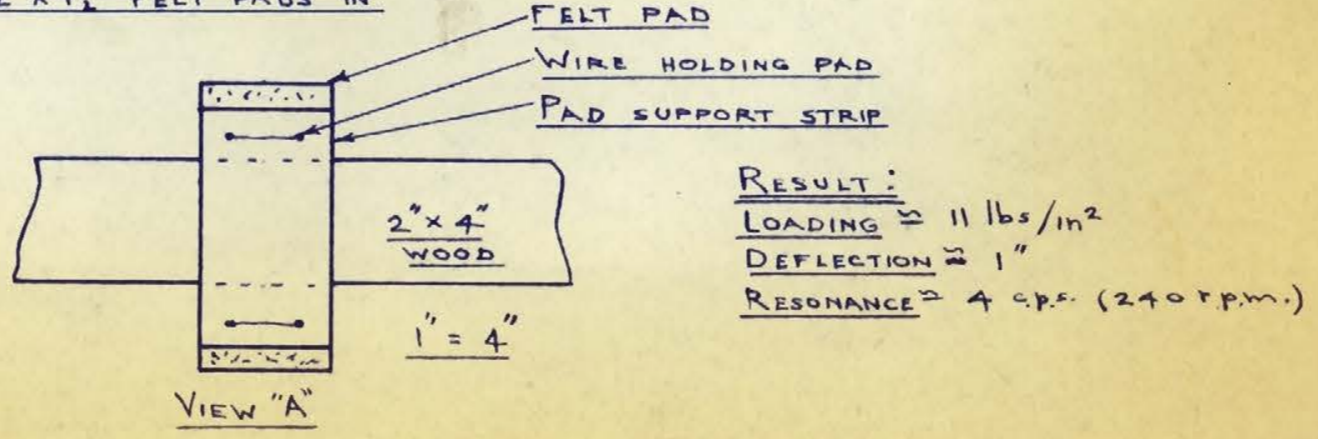


the floor using this base, but it was discarded for reasons given in Art. 5. It was desired to mount the fan on a concrete base, but facilities would not permit.

2. An anechoic chamber was used to measure PWL and spectra of the fan runners rotated alone. Fig. 3.3(a) shows the drive and measuring instruments exterior to the anechoic chamber, while Fig. 3.3(b) shows the interior. The room is $14^{\prime} 6^{\prime \prime}$ long, 9'2" wide and $10 '$ high. Walls, outside to inside, are of plaster, 5" hollow clay blocks, 4 inch space and 4 inches of $21 / 2 \mathrm{lbs} / \mathrm{cu} . \mathrm{ft}$. PF fiberglas. No tests on the room were conducted except a bandwise check of reduction in SPL with distance from the rotating fan runner as source. Fig.5.15a shows this to be quite good. Some standing waves are known to occur below 500 c.p.s.

3. The room in which the system of Fig. 1.3 was installed is 37'long, 16' wide and 12'9" high. It is of plasterboard construction on two internal walls, brick and glass on two external walls, concrete ceiling and wood on concrete floor. As such it is a fairly "live" room acoustically. The reverberant sound field was always about $20 \mathrm{db}$ below that of sound inside the acoustic waveguide. Overall ambient sound level of the location generally ranged between 50 and $60 \mathrm{db}$. re $.0002 \mu$ bar. 4. The Pitot tube and manometer are shown in the foreground of Fig. 3.2a. The Pitot tube is of standard construction and conforms to that design recommended on page 1105, Ref.54 recommended by the American Society of Heating and Ventilating Engineers. The manometer consisted of two l' long, 0.2" bore glass U tubes connected by short lengths of rubber hose to the Pitot tube. A graduated scale gave readings direct to $0.1 \mathrm{~N}_{2} \mathrm{O}$ 
estimated to $0.01 " \mathrm{H}_{2} \mathrm{O}$. The tube for static pressure was vertical while that for measuring velocity pressure was inclined $15^{\circ}$ to the horizontal. Air velocity is then:

$U=32.8 \sqrt{h_{w \text { (observed) }} \times \frac{.76}{P_{0}} \times \frac{T}{492}} \mathrm{ft} / \mathrm{sec} . \quad \cdot \quad 3.1$

$h_{w}$ (observed) $=$ head in inches of water observed directly on the inclined manometer

Po $=$ Ambient air pressure, meters of Mercury

$\mathrm{T}=$ Absolute temperature converted to ${ }^{\circ} \mathrm{F}$

Distortion of the meniscus was found to have negligible effect on the reading of the inclined tube. A micro-manometer served to check this manometer. Levelling screws and zero set were part of the equipment.

5. A Mercury barometer having an accuracy of $\pm 0.01 \mathrm{~cm}$. of mercury was used to measure barometric pressure. Temperatures were recorded to the nearest ${ }^{\circ} F$ using a standard laboratory Mercury themometer which had been checked against two others. Relative humidity was determined to an accuracy of about I 1\% using a Taylor 5532-s Hygrometer. 6. Connecting cables were coaxial. When specified for proper operation, instruments were well grounded to a low impedance system and were placed on a grounding plate.

7. Power supplies were:

(a) $115 \pm 5 \mathrm{~V}, 60 \pm 1$ c.p.s.

(b) $115 \pm 5 \mathrm{~V}$, d.c. no load, $105 \pm 5 \mathrm{~V}$, d.c. at motor full load (50 amps.). 


\subsection{EQUIPMENT PERFORMANCE AND ESTIMATED ACCURACY}

\section{2 .1 PERFORMANCE}

The matched acoustic waveguide was discussed in detail in Art. 2.1. Experimentally, its performance is represented by Fig. 2.2 a. Beyond the range of this graph, refer to the text of 2.1 .

The General Radio Sound-Measuring System was checked and operated in accordance with the appropriate operation manuals provided with the equipment listed in Art. $3.1 \mathrm{C}$. The overall acoustic calibration at $400 \mathrm{c} . \mathrm{p} . \mathrm{s}$. was performed at least weekly, the electrical circuit calibration for the sound level meter was checked every day readings were taken. These calibrations generally agreed within $\pm 1 \mathrm{db}$. The equipment was carefully handled, was not used on any other project during all but preliminary measurements and is relatively new. For these reasons, the manufacturers tolerances are presumed to be met (see Art.3.2.2).

Perhaps the most annoying and inaccurate equipment behaviour was poor voltage regulation of the d.c. supply to the motor. Other loads on the same cable system caused instantaneous voltage variations up to 5 volts, which represents a change of fan speed of about 5\%. The photo electric pick up and counting circuit, giving direct visual indication of the speed at every instant was an immeasurable asset in determining average speed.

Fan and motor vibration did not affect the measurements. Although vibration was transmitted directly to the floor, the mass 
of the concrete floor prevented significant vibration from reaching the measuring location or the duct downstream from the canvas break. The manometer was mounted on a pedestal and was insulated from it by pads so that there was no tremor of the meniscus.

The performance of the windscreen is illustrated by Fig. 3.7. This data was supplied courtesy of Mr. A. Bredahl6. The attenuation curve (Fig.3.7a) was obtained by measurements in an anechoic room while the wind noise attenuation (Fig.3.7a) was determined by rotating the microphone in a wide circle using special apparatus ${ }^{6}$. Comparison between overall values with and without windscreen at the duct microphone station of Fig. 1.3 is shown in Fig. 3.7b. Bandwise, Mr. Bredahl found the difference in noise level between a screened and unscreened microphone varied between 7 and $20 \mathrm{db}$, the reduction for different air speeds ( 30 to $60 \mathrm{ft} / \mathrm{sec}$ ) being relatively constant in each band. As mentioned in Art. 2.3, and as substantiated in Art. 5, the effect of the turbulent air stream over the windscreen, on the signal output from the microphone has not been entirely eliminated. Nonetheless, the designs by Mr. Bredahl appear to be among the best to date.

\subsubsection{ESTIMATED ACCURACY OF THE EQUIPMENT}

In this section, instrumental and observational errors are evaluated; to be used at the end of Art. 5 for computing overall errors in the results. 
Effectiveness of Triple Spherical Windscreen Used

$$
\text { (After Mria Brednhí }{ }^{\circ} \text { ) }
$$

(a) Windscrein Performance

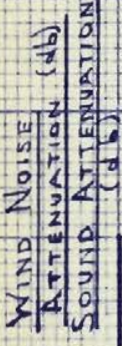

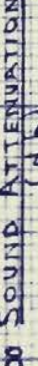

308

20

- Wing Noise Attenyatipin of

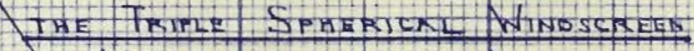

(actaye band nescissa)

10

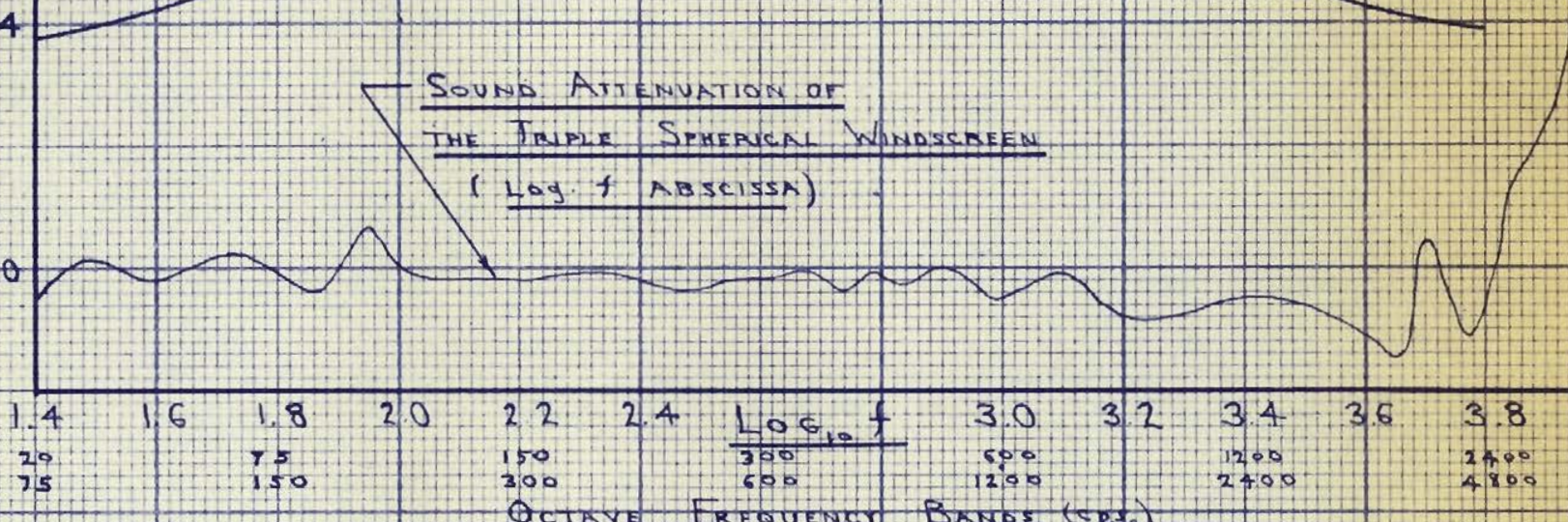

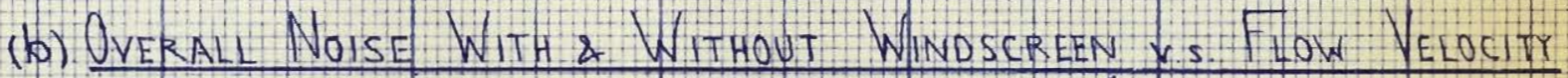

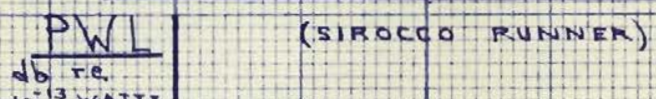

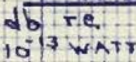

120

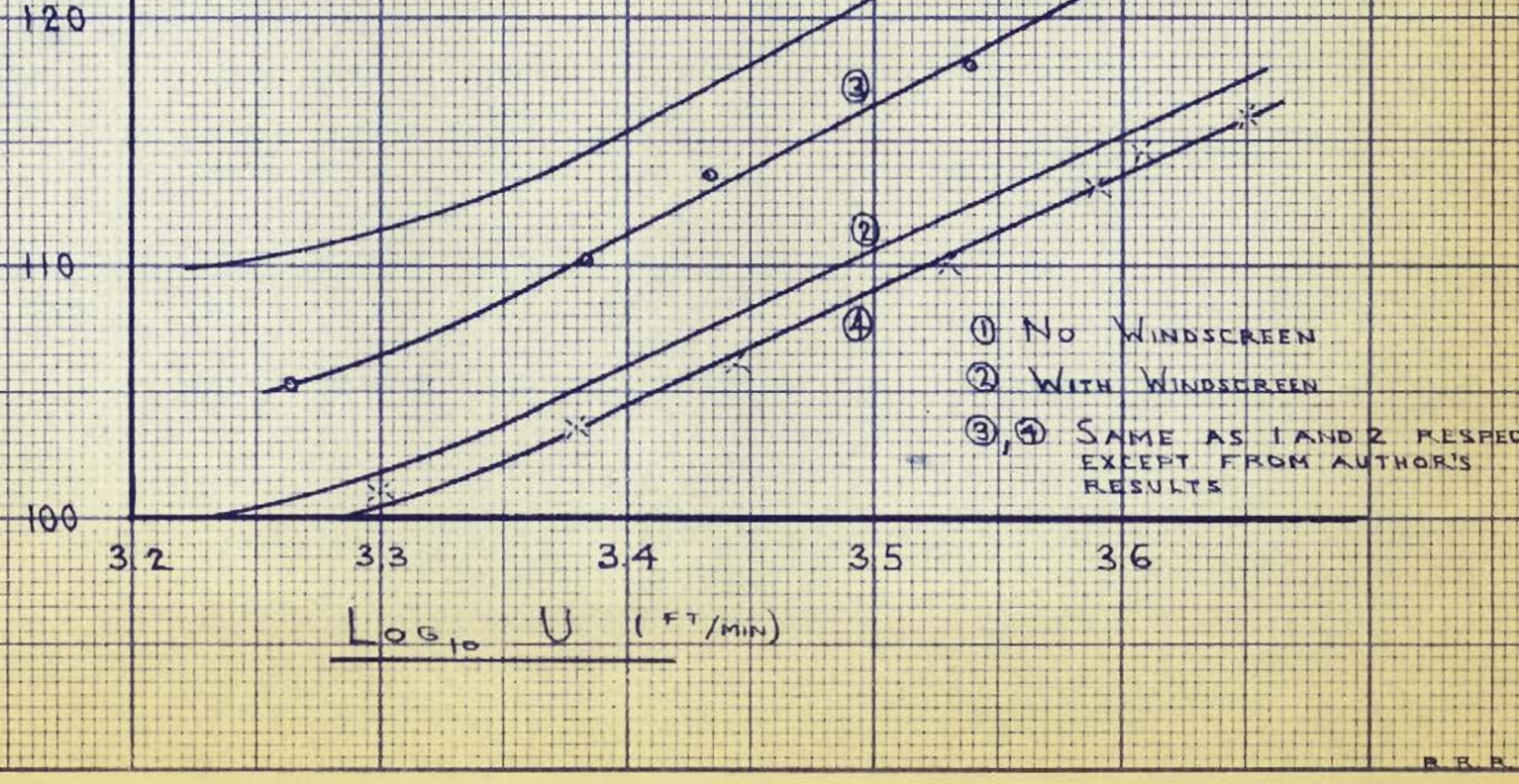




\section{INDEPENDENT SOURCES OF ERROR}

\section{GENERAL RADIO SOUND MEASURING SYSTEM}

Calibration of the Sound Level Meter (SLM) and microphone was performed with the 1552 B calibrator. This provides for an overall operational check of SIM and microphone at 400 c.p.s. This acoustic calibration and electrical calibration were performed at least weekly and always agreed to within $\pm 1.5 \mathrm{db}$. The electrical circuit calibration was performed at least daily during accumulation of measured data. This, plus other reasons given in Art. 3.2.1 are believed sufficient evidence to verify that the condenser microphone system and SIM are together within the A.S.A. tolerances ${ }^{64}$ :

\section{TABLE 3.1}

$\begin{array}{cc}f & \text { Tolerances }(d b) \\ 25 & -6,-9.5 \\ 60 & -3,-3 \\ 300 \text { to } 1100 & -2,-2 \\ 2000 & -3,-4 \\ 3000 & -4,-5.5 \\ 5000 & -5,-7.5 \\ 8000 & -6,-9.5\end{array}$

The performance of the microphone is most uniform at the lower freqencies $(<1500 \mathrm{c.p.s}$.$) where most of the noise$ power measured exists.

Observational error is on the order of $\pm 1.5 \mathrm{db}$, on the "Slow" meter position (heavy meter damping). Fluctuations were usually \pm 3 or $4 \mathrm{db}$, hence a short time average of each reading was necessary to minimize the error.

The $760-B$ 2\% B.W. Sound Analyser was used extensively for spectra measurements. Its frequency response was checked 
and found to be $\pm 2 \mathrm{db}$ from 25 to 2500 c.p.s. and $t 3 \mathrm{db}$, -I db from 2500 c.p.s. to 7500 c.p.s. (There is negligible power in this last band.) Observational error was $\pm 2 \mathrm{db}$ down to a level of $25 \mathrm{db}$ below the overall sound level. Fluctuations of the meter needle of \pm 1 to $\pm 6 \mathrm{db}$ were common in any one spectrum due to the narrow bandwidth, and fan rotor speed and turbulence fluctuations. Method of obtaining the spectra is discussed in Art. 4.

The 1550 - A Octave Band Analyser was used for a few spectra, plus noting the bandwise variation of fan noise with speed and position of microphone in the duct section. The bandwise filter errors for random noise are about $\pm 1 \mathrm{db}$, from 75 c.p.s. to 4800 c.p.s. The low pass filter $(20-75$ c.p.s.) would give an indication of $1 \mathrm{db}$ lower than the other bands, provided it cut off sharply below $20 \mathrm{c.p.s.,} \mathrm{while} \mathrm{the} \mathrm{high}$ pass filter ( 4800 to 10,000 c.p.s.) would be about 2 db lower. This is partly compensated for (indeed it may be overcompensated in some cases) by noise power below 20 c.p.s. and above 10,000 c.p.s. For our purposes, the Octave Band Analyser will be considered as having an inherent bandwise error of $\pm 1 \mathrm{db}$.

\section{STANDING WAVES IN THE MATCHED ACOUSTIC WAVEGUIDE,}

\section{AND WIND NOISE}

These were discussed in Art. 2.1. The method of correcting for transverse variations due to the higher modes is discussed in Art. 4. The serious error here is in the low band, 20 to 75 c.p.s., below horn cut - off. As pointed out in Art.2.1, the error will be low by about 15\% of the pressure 
being measured, provided white random noise occurs throughout this band. This latter is not true, but in most cases is roughly approximated. What then does such an error mean in terms of $\mathrm{db}$ :

$$
\triangle S P L=20 \log \frac{P_{1}}{P_{2}}=20 \log \frac{.85 P_{2}}{P_{2}}=-1.5 \mathrm{db}
$$

In view of the assumptions dealing with this in Art. 2.1 , the frequent presence of one line component in this band ("rotational" component, r.p.m./60 c.p.s.) and the fact that the noise is not white, an error of say $+1,-3 \mathrm{db}$ should be specified due to standing waves in the low band. In turbulent air flows however, a windscreen covered microphone such as the one mentioned in Art. 3.2.1 does not attenuate all the locally generated noise due to its presence, for reasons stated in Art.2.3. Further research is required to evaluate this. There is reason to believe that it is the low frequencies generated locally which contribute the most to this in air flows of ventilating Rey. numbers. This will tend to boost the measured SPL in the 20 to 75 c.p.s. band. Because of the unknowns, and the fact that this windscreen design was among the best (see Ref.6 for review of other papers on windscreens), it is assumed (ref.6) that although wind noise is not eliminated, it is minimized to insignificance compared to the SPL measured in these tests on fan noise.

\section{AIR FLOW AND PRESSURE}

Velocity pressure was read on an inclined tube manometer ( $15^{\circ}$ to horizontal) with a least scale reading of 0.1 " and an estimated scale reading of $0.01^{\prime \prime}$ (with the aid of a 
magnifying lens). Observational error due to fluctuations in the flow is estimated as follows:

Above I" $\mathrm{H}_{2} \mathrm{O}$ velocity head: $0.02 "$

Between 0.5 and $1^{11} \mathrm{H}_{2} \mathrm{O}$ velocity head: $0.01 "$

Below $0.5 \mathrm{~N}_{2} \mathrm{O}$ velocity head: $0.005 "$

A further error of $0.01^{\pi}$ is due to adhesion of the meniscus to the tube wall, the manometer not being precisely level, and temperature variations up to $\pm 5^{\circ} \mathrm{F}$. Converting to equivalent inches of water, the errors are:

$$
\begin{aligned}
& \mathrm{h}_{\checkmark}>\text { I" } \mathrm{H}_{2} \mathrm{O} ; 0.010 \text { " } \mathrm{H}_{2} \mathrm{O} \\
& \text { I" } \mathrm{H} \mathrm{O}>\mathrm{h}_{\checkmark}>0.5 " \mathrm{H}_{2} \mathrm{O} ; 0.0008 " \mathrm{H}_{2} \mathrm{O} \\
& \mathrm{h}_{\vee}<0.5^{n} \mathrm{H}_{2} \mathrm{O} ; 0.007^{n} \mathrm{H}_{2} \mathrm{O}
\end{aligned}
$$

The static pressure $\left(h_{s}\right)$ manometer was vertical. Fluctuations were negligible, hence $0.005^{\prime \prime}$ will be assumed due to these. The two $0.01 "$ errors for $h \vee$ also apply here, hence the error for $h_{s}$ is $0.025 " \mathrm{H}_{2} \mathrm{O}$.

\section{SPEED}

Fan speed could be read to \pm 1 r.p.m. by the photo multiplier - counter circuit. During the course of a run however, line voltage fluctuations would cause instantaneous changes in speed of up to $5 \%$ but usually within $3 \%$. By recording and averaging the speed several times per run, this error was reduced to an estimated $\pm 1.5 \%$.

\section{POWER TO FAN}

This was estimated from input power readings using the efficiency curve, $3.8 \mathrm{a}$ and the performance curves $3.8 \mathrm{~b}$. (These 


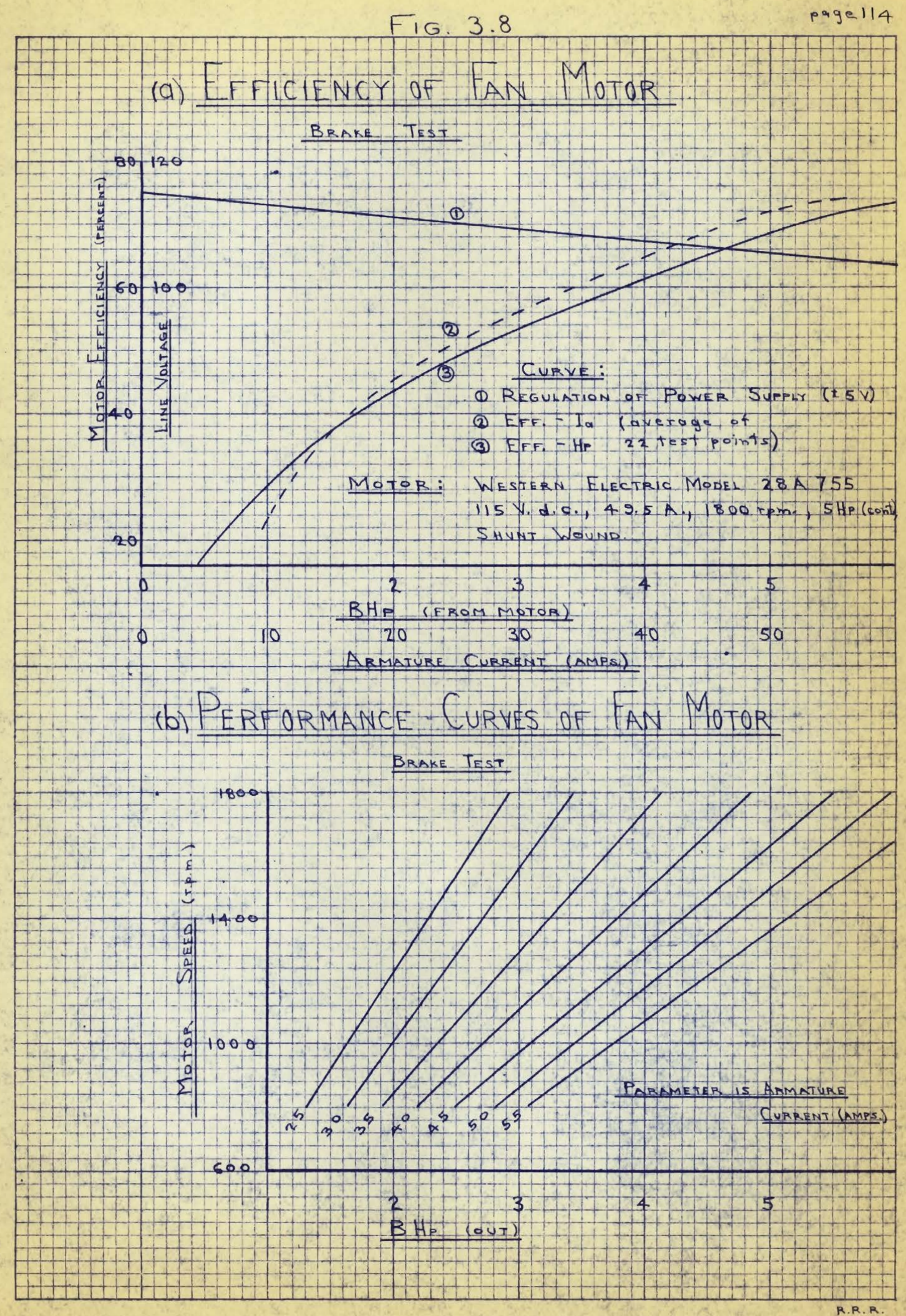


of course have been reduced in scale here to save space.) Where the family of performance curves did not apply, the efficiency curve was used (mainly at low values of speed and high system friction (back pressure) . These curves were obtained from a brake test having the following errors:

Input Power: $E_{\alpha}, E_{f}: \pm 2 V$ and $\pm 1 V$ observational.

$I_{a}: \pm 2 \mathrm{~A}$ and $\pm 0.5 \mathrm{~A}$ observational.

$I_{f}: \pm 0.05 \mathrm{~A}$ and $\pm 0.01 \mathrm{~A}$ observational.

$\pm \Delta$ Win $= \pm \Delta\left[E_{a} I_{a}+E_{f} I_{f}\right] \simeq \pm 10$ watts.

Subscript "a" refers to armature, "f" to shunt

field.

Output Power:Torque: $\pm 1.5 \mathrm{ft.lbs}$. and $\pm 0.5 \mathrm{ft.lb}$. observational.

r.p.m. $\pm 1 \%$ and \pm 10 r.p.m. observational. (strobotac)

$\pm \Delta H p$ out $= \pm \Delta[K(T)(r \cdot p \cdot m \cdot)]$

(function of speed)

The family of curves Fig. $3.8 \mathrm{~b}$ was necessary because speed was varied by regulating armature voltage, and motor efficiency depends on $E_{a}$ as well as load.

$$
\text { Shunt motor efficiency }=\left|\frac{E_{a} I_{a}-\left(I_{a}{ }^{2} R_{a}+K_{1}\right)}{E_{a} I_{a}+K_{2}}\right|_{n=\text { constant }}
$$

where $R_{a}$ is the armature resistance

$K_{1}$ represents the constant losses

$\mathrm{K}_{2}$ represents input to the field (constant except for voltage regulation).

At rated full load, rated voltage, $K_{1} \cong I_{\alpha}^{2} R_{a}$ while below this $K_{1}>I_{\alpha}{ }^{2} R_{a}$. One is most interested in Hp to $f$ an and speed of same, while the main varikles are $E_{\alpha}, I_{\alpha}$. For a constant speed, neglecting $I_{\alpha} R_{\alpha}<E_{\alpha}$, Speed $n \propto E_{\alpha}$, and the efficiency is relatively constant. To vary the speed however, $E_{a}$ must be correspondingly varied and also $I_{\alpha}$ will vary: 


$$
\begin{aligned}
& E_{\alpha}=K_{4} n+I_{\alpha} R_{\alpha} \\
& \text { hence } I_{a}=\frac{E_{\alpha}\left(I-K_{5}\right)}{R_{\alpha}} \text {, since } n \alpha E_{a}
\end{aligned}
$$

$\mathrm{K}_{s}=$ constant dependent on motor design

The rotational losses (formerly represented by $K_{1}$ )

are now variable:

Friction and windage $\propto n^{2-3}$

Hysteresis loss $\alpha \mathrm{n}$

Eddy current loss $\propto \mathrm{n}^{2}$

Resulting Variation $\approx E_{\alpha}^{2}$ say.

Eff. $=\frac{E_{a}^{2}\left(k_{c}-k_{c}^{2} R_{a}-k_{7}\right)}{E_{a}^{2} k_{c}+k_{2}}$

$K_{c}=$ constant $\frac{\left(1-k_{s}\right)}{R_{a}}$

$\mathrm{K}_{7}=$ constant associated with the rotational losses.

$K_{2} \cong E_{+} I_{+} \simeq$ constant.

As $E_{a} \longrightarrow 0$, Eff. $\rightarrow 0$. If $E_{\text {line }}$ fluctuates, or if

$E_{a}$ is reduced for speed regulation, efficiency can assume the following values (having evaluated the above constants) relative to that at rated applied voltage of 115 volts:

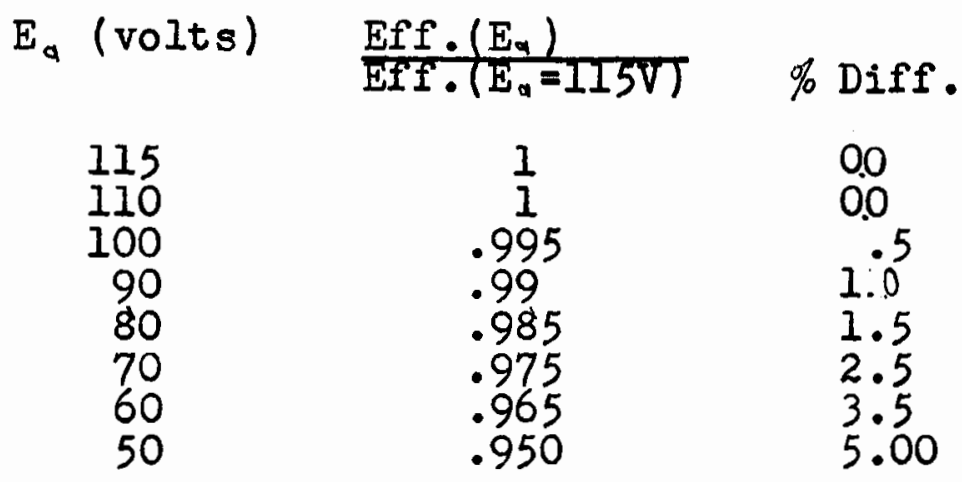

This represents the approximate range of armature voltage used for speed regulation in this project. For the reason implied by the above table, and for convenience, Fig. $3.8 \mathrm{~b}$ was drawn, using a large number of carefully taken brake test readings. The brake test is inherently not the most accurate test that 
could be used, but was the most convenient.

The Hp input to the $f a n$ is in error by the following estimated percentages, allowing $\pm 1 \%$ error in the assumed $2 \%$ power loss of the $V$ - belt drive:

TABIE 3.2

\begin{tabular}{|c|c|c|c|c|c|c|}
\hline Hp to fan & 1800 & $\begin{array}{l}\text { MOTO } \\
1600\end{array}$ & $\begin{array}{c}r \cdot p \cdot m \\
1400\end{array}$ & 1200 & 1000 & 800 \\
\hline $\begin{array}{l}6 \\
5 \\
4 \\
3 \\
2 \\
1 \\
.5\end{array}$ & $\begin{array}{l}8.5 \\
9.5 \\
11 \\
14 \\
20 \\
37 \\
70\end{array}$ & $\begin{array}{l}7 \cdot 5 \\
8 \cdot 5 \\
10 \cdot 5 \\
13 \\
19 \\
34 \\
65\end{array}$ & $\begin{array}{l}7.0 \\
8.0 \\
9.5 \\
12 \\
17 \\
30 \\
60\end{array}$ & $\begin{array}{l}5 \cdot 5 \\
7 \cdot 5 \\
8.5 \\
11 \\
15 \\
26 \\
50\end{array}$ & $\begin{array}{l}6.5 \\
7 \cdot 5 \\
9 \cdot 5 \\
13 \\
24 \\
40\end{array}$ & $\begin{array}{l}- \\
\overline{6} .5 \\
8.5 \\
11 \\
19 \\
35\end{array}$ \\
\hline
\end{tabular}

The results of the foregoing are applied in "Accuracy of the Results", end of Art. 5 . 
4. PROCEDURE

The basis of the numerous graphical results was the accurate measuring and plotting of the following curves:

1. Air flow (U) vs. Fan rotor speed (n) with constant system ( $\left.F_{\text {syst }}\right)$ as the parameter.

Fsyst was varied by changing the relative position of the drilled back panels (Fig.3.5), thereby changing the relative system friction or back pressure (a function of air velocity) as "seen" by the fan. The back panel was calibrated for seven settings giving fairly evenly spaced curves ranging from low Fyst to high $F_{\text {syst }}$.

2. Static pressure corrected to fan outlet (hs) vs. Fan rotor speed $(n), F_{\text {syst }}$ parameter.

3. Hp to fan (Hp in) vs. Fan rotor speed, $F_{\text {syst parameter. }}$ 4. Overall PWL vs. Fan rotor speed, F $F_{\text {syst }}$ parameter. From these four basic families of curves (which must be repeated for each different fan tested), the variation of overall noise intensity with fan speed and $\mathrm{Hp}$, air flow velocity, capacity, static pressure (back pressure), and variable $F_{\text {syst }}$ can be determined. This forms one series of results.

The other major portion of the results is isolation of the noise origins by taking extensive noise spectra. 


\section{I MEASUREMENTS}

\subsubsection{FLOW - PRESSURE - HP - SPEED DATA}

Measurements of air flow velocity, static pressure and Hp to fan vs. rotor speed, with $F_{\text {syst }}$ as parameter were taken together. For each setting of $F_{\text {syst }}$ (back panel) and fan speed, 20 readings of $h_{v}, h_{s}$ were taken in the duct cross section. These stations are on two mutually perpendicular diameters according to the Heating, Ventilating and Air Conditioning Guide ${ }^{54}$ p.1105, 1954 (NAFM code). The arithmetic average of these gives $h_{v}$ and $h_{s}$ for one $F_{\text {syst }}$ and speed. Actually, a 21st reading was taken at the duct center for comparative purposes. On the average it gives readings of $h_{\vee} 3$ to $10 \%$ high (depending on $U)$ and readings of $h_{s}$ close to the average. Guides assured the Pitot tube was parallel to the axis. The manometers were read with the aid of a magnifying lens. A time average of $h_{v}$ was taken for higher flow velocities because of some fluctuation of flow at a given station.

Readings of fan speed and motor input $E_{a}, I_{-}, E_{f}, I_{f}$ were taken and averaged for each point. Employing Fig. 3.8 (in its original enlarged form) gave Hp to fan.

The above was done for speeds of $800,1000,1200,1400$, 1600 and 1800 r.p.m. for the sirocco fan, double these for the "HS" fan, for each of the seven Fsyst positions. Once learning $h_{v}$, hence air velocity, a correction to $h_{s}$ could be made for the length of duct and mesh between fan and Pitot tube. After correcting for temperature and pressure, which were recorded regularly each run, curve families 1 to 3 above were accurately plotted and drawn. 
4.1.2 PWL VS. SPEED

The fourth "basic" family of curves is overall $f a n$ noise PWL - Speed. The general procedure is the same as above, except speed increments were generally half those above and the windscreen covered microphone was kept only on the duct axis. (Further sets of measurements later provided bandwise corrections for SPI variations across the duct section, see Art.4.1.5) For each $F_{\text {syst }}$ and speed, overall SPL, octave bands, motor input and speed were read, speed being the average of several readings displayed by the counter. Because of the random nature of the noise and turbulence in the flow, a time average of all overall SPL and some octave band readings was necessary. Electrical calibration of the SIM was made frequently, acoustical calibration at $400 \mathrm{c.p.s}$. at least weekly, and setting of the Octave Band Analyser gain was checked for each $F_{\text {syst }}$ and speed. Pressure and temperature were recorded. Overall PWL $\simeq$ SPL for this duct section of $1.05 \mathrm{sq}$.ft. were plotted vs. speed. Checks were made of the plotted points, and where agreement was not within $\pm 1 \mathrm{db}$, further measurements were made and the results averaged. Generally this was not the case.

The microphone was oriented $90^{\circ}$ to the noise source for all measurements in this research. Such orientation results in the generally best gain - frequency characteristic for the condenser microphone - SIM system. 


\subsubsection{NOISE SPECTRA}

Most spectra were taken with the G.R. 760 - B 2\% B.W. Sound Analyser. It is felt this analyser is the most useful, since a band analyser does not reveal prominent "line" components, while a constant B.W. analyser is too highly selective at higher frequencies. Speed regulation and noise fluctuations do make it rather difficult to use the $2 \%$ B.W. Sound Analyser in detail. Its use was as follows: Starting at 25 c.p.s., the tuning dial was very slowly turned over small increments of frequency. Over this range, sound fluctuations were observed. If a "line" component was suspected by watching the meter fluctuations, it was found and its maximum average and minimum average value noted. If there was a sudden change in the sound level, the dial would be turned to retrace the band over which the sound level was approximately constant. The tuner would then be approximately centered in this band and a time average taken. With practice, a spectrum 25 c.p.s, to 7500 c.p.s. (the limits of the 760 - B Analyser) could be "swept" in 20 to 30 minutes. This may appear rather slow, but is much faster than a Wave Analyser (constant B.W.) and gives spectra which are as detailed, especially if much of the noise analysed is low and mid-frequency. The writer firmly belives the Sound Analyser to be the most useful for procuring meaningful, detailed noise spectra, provided it is carefully used. One-third octave bands will be close to giving the shape of a continuous spectrum, but will not measure line components. The octave band analyser is useful for preliminary spectra measurements and checks, and field measurements but is not adequate for spectra measured in a laboratory. 
All G.R. Sound measuring equipment was operated, checked and maintained in accordance with the respective instruction manuals.

\subsubsection{VIBRATION}

Extensive fan vibration measurements were taken using G.R. Type 759 - P35 crystal pickup and the G.R. Type 759 - P36 Control Box in conjunction with the SLM, Octave and $2 \%$ BW Analysers. Preliminary measurements were taken on various parts of the fan. It was decided from these that the most useful detailed results would come from the upper side of the fan outlet (Fig.2.4 e,p.7.). The vibration pick-up was bolted to the position shown for most of the fan vibration measurements. This would give all the housing vibration components in the direction of the duct. Lateral ones, though present were small and were considered to have negligible effect on the "vibration noise" in the duct.

Vibration measurements were made on the duct at the microphone position and on the floor near the fan. The vibration at these points proved small (see results).

If the microphone was at a station where vibration could be felt, the signal due to this was determined by applying a tight fitting cap over the microphone and observing the bandwise output. In all cases and bands the signal was at least $15 \mathrm{db}$ (usually more) below that when the sound was being measured. The attenuation of the cap was not measured, but it is unlikely it exceeds $20 \mathrm{db}$ at the lower frequencies. This was done for all microphone stations in the research, the results 
showing that any vibration the microphone was subjected to due to transmission through the floor to the apparatus and from the turbulence in the airstream had negligible effect on the results.

\subsubsection{STANDING WAVES}

In addition to the analysis of Art. 2.1, means were taken to minimize the error due to standing waves along the duct and across it. Bandwise measurements were first made with a random noise generator (amplified resistor noise, not listed in the Apparatus) as source. The microphone could then be moved from the duct center to the periphery, since a windscreen was unnecessary. This was done for two mutually perpendicular diameters across the duct section. The same stations were used as for flow measurements. Measurements were also taken longitudinally with the microphone on the duct axis. This was then repeated with the windscreen covering the microphone, for each of the fans operating at various flow conditions (eg. Four settings of $F_{\text {syst }}$ and three speeds). The results are in Art.5. The similarity of results between the random noise generator and fan allowed extrapolation into the region the microphone could not be extended to because of the windscreen surrounding it. In all cases, bandwise plots of SPI vs. distance across the duct were made. From these, corrections were estimated and applied to the octave band readings taken during the overall PWl noise measurements with the microphone on the duct axis at the station indicated in Fig. 1.3 p.18. 


\subsubsection{TERMINATION STUDY}

Contour maps of air flow and SPL were taken just beyond the horn mouth, as shown in Fig. 3.5. Readings of $h_{v}, h_{s}$ and SPI were taken at 16 stations, representing the centers of 16 equal squares into which the termination cross section was divided. Additional measurements were made near flow and SPL maxima. This was done for various fan operating conditions. Spectra were also taken here, at stations indicated in the results.

\subsubsection{ANECHOIC ROON}

Noise spectra and overall PWL measurements were made on the fan runners rotating in isolation in the anechoic room. All the equipment was kept exterior to the room except the microphone, runner and one pillow block and support, as $\mathrm{c}$ an be seen in Fig. 3.3. Overall PWL was determined by measuring SPI at the centroids of surfaces represented by a quarter of a sphere divided into 3 and 5 equal parts. This was done for radii from the runner center of 3 and 5 feet. The results are discussed in Art. 5. Spectra were measured using the $2 \% \mathrm{~B} . \mathrm{W}$. Analyser, and checked by the octave band analyser. No ventilation is provided in the room, and with fan runners of the size tested running near top rated speeds, temperature rose as much as $20^{\circ} \mathrm{F}$ in a 30 minute period due to air turbulence. Rough temperature averages were considered sufficient considering the accuracy of the measurements. 


\subsection{PREPARATION OF RESUITS}

4.2.1 FAN NOISE LAWS

These curves refer to overall noise PWL versus various fan - flow parameters. They werepresented as families of curves with some parameter held constant. Each point corresponds to one particular setting of the back panels. Many preparatory pages of curves were drawn to aid in presenting the results in the most rational form. By plotting all parameters on a $\log _{10}$ base, fan "noise laws" became apparent. No sample calculations are presented here, because they are merely the elementary application of simple fan and acoustic formulae which have been given elsewhere in this thesis. Further elaboration is given in Art. 5 as necessary.

\subsubsection{FAN NOISE SPECTRA}

The results measured with the $2 \% \mathrm{~B} . \mathrm{W}$. Analyser were computed from the overall SPI reading as per instruction manual for the instrument. It has been mentioned that the average maximum and average minimum values of reading on this meter were noted. The SPI of a band $2 \%$ wide was taken as the arithmetic average of these two readings, provided their difference $<10 \mathrm{db}$. If their difference $>10 \mathrm{db}$ (as it was occasionally), the average value was taken as the minimum average plus $2 / 3$ of the difference between the minimum average and the maximum average. (This is almost the same as subtracting $3 \mathrm{db}$ from the maximum average, as recommended in Ref.62). As described in Art.4.1.3, the spectrum was slowly "scanned" with the $2 \% \mathrm{~B}$.W. Analyser in such a manner that the SPL recorded represented the average maximum and average minimum in a $0.02 f$ band, where $f$ is the cen- 
ter frequency of a band $\Delta f$ over which the average maximum and average minimum were approximately constant (within 1 or $2 \mathrm{db}$ ). Each plotted point in a spectrum taken with this instrument therefore represents a band of frequencies $\Delta f$ in which the average maximum and average minimum SPL in a band $0.02 f$ ( $f$ being the center frequency) is nearly constant. Note that it does not represent the sum of SPI in a band $\Delta f$. Line components are plotted as such along with the spectra.

The most universal method of plotting the spectra would have been to reduce the SPI to their pressure spectrum levels in $\mathrm{db}$ (eg. reduce them to a bandwidth 1 c.p.s. wide) and plot this against $f / f \max$ as abscissa. This was done in only one case. The reason for not doing them all is this: The $2 \%$ B.W. Sound Analyser is believed by the writer to be the best current basis for intercomparison of spectra of wideband random noise containing "line" components. This instrument gives a maximum of laboratory spectral information in a minimum of time; and this information is a minimum for noises such as fan noise because they are not only wideband and random, but are likely to contain a few line components. If this instrument receives the widest acclaim for laboratory measurement of fan noise at least, then less time is consumed by all if intercomparison of these data are made of the basis of a $2 \% \mathrm{~B} . \mathrm{W}$. Sound Analyser sweeping the spectrum. Further ideas along this line are mentioned in Art. 7 .

Theoretically, these spectra should be corrected for standing waves at both sub horn cut - off frequencies and frequencies at which the higher modes present themselves. Such a 
task has not been attempted here. Because of meter damping and the averaging method used to obtain the plotted data, the resulting spectra are believed to give a fair indication of things as they really are.

Octave band spectra were corrected for transverse variations according to Art. 4.1.5. The average bandwise SPL as computed from the corrected minimum average and maximum average readings were then plotted. In many cases when the spectrum was declining rapidly, readings in the upper four bands were taken using the $A$ and $C$ networks of the SIM and the correct reading derived therefrom as stipulated in the instruction manual for the octave band analyser. In all cases this was done for the $4800 / 10,000$ c.p.s. band. Special procedures in the instrument manuals must be adhered to rigidly if reliable results, unadulterated by self noise of the instrument and such, are to be obtained.

\subsubsection{VIBRATION SPECTRA}

Octave band and $2 \% \mathrm{~B} . \mathrm{W}$. spectra were taken of particle acceleration and velocity from 20 c.p.s. to about 1100 c.p.s., at which point the pick-up crystal response coupled with the control box and SIM becomes very non linear. These results were taken in the same way as the noise spectra. They were first corrected for no linear response using Fig. 7 Ref.63, before computing acceleration and velocity from the voltage $\mathrm{db}$ conversion tables Ref.63. 


\section{RESULTS AND DISCUSSION}

Noise origin, power and spectra of ducted centrifugal fans has been studied in detail. It was mentioned that the most important factor in acoustics is that the human brain can judge sound intensity and its spectrum at the ear. Overall acoustic power level and its spectrum, plus directivity information, completely describe an acoustic source. Separating source and ear is an acoustical network; as simple as free space, as complex as the ventilating system in a large building. Whether it be music or noise that is involved, the acoustician will first study and modify the source, then the network medium, and finally the vicinity about the ear, in that order in any acoustical problem. The results herein add to the knowledge of one type of noise source; the commercial ducted centrifugal fan.

The nature of noise is such that much data is required to obtain the smallest concrete result. It is therefore economically desirable for the fan manufacturer to either avoid fan noise measurements or at least keep them to a minimum. On the other hand, the customer should be given sufficient information to roughly assess noise intensity and frequency distribution at any given point relative to the noise source. Ventilation and acoustical engineers are interested in both "source" and "load" specifications so that they can design the intermediate network accordingly. The simplest possible fan noise information is overall PWL at a given point of rating. This is insufficient. Since the ear can judge spectra to an extent, frequency distribution of the source is essential. For ducted 
fans in separate rooms remote from the listener, directivity is fixed in the sense that one is concerned only with the noise propagating down a duct. To have noise data for only one point of rating is impractical, since the fan may operate under varying load conditions, or never operate on the point of rating specified.

What the fan manufacturer and ventilating or acoustical engineer should have at their disposal is a system of estimating the overall PWL of a given fan operating at a given point, then tag this PWL with an appropriate spectrum. These would not be precise, but would provide probable information regarding the acoustic source. More will be said in Art. 7 . The present task is to establish a universal technique for determining PWL and its spectrum for a given fan operating under a given condition.

The results herein are presented and discussed under the three broad headings of overall noise power laws, noise spectra and origin, and finally, other studies. They are summarized in Art. 6. The graphical data is arranged so that the Sirocco fan results always precede the HS fan results.

\subsection{OVERALL FAN NOISE POWER}

In the review of the literature, Art. 1.I, many empirical formulae relating fan PWL with fan and flow parameters are given. Data is conflicting. For the most part the "laws" evolved are from PWL vs. U, h r.p.m. and Hp (see glossary of 
symbols) with constant system (Fsyst) as parameter. The results presented here will exhibit further conflict. However, the author has gone a step further by accumulating a vast amount of measured data and organizing it to a high degree so that the most useful combinations of fan - flow - noise parameters are subjected to careful analysis. From this, a universal method of predicting ducted centrifugal fan noise output PWL is evolved.

\subsubsection{PRELIMINARY CORRELATION}

The first set of curves, Fig. 5.la, b (a for the Siro$c c o, b$ for the HS) consists of various fan and flow parameters plotted against air velocity, for constant speed. The symbols are repeated here for convenience (they can also be found in the glossary of symbols):

Air Power $=1.575 \times 10^{-4} \mathrm{Qh}_{\mathrm{s}} \times 746$ watts.

Hp - Horse power to fan rotor.

$h_{s}=$ Static pressure of flow, inches of water.

$I$ = Sound intensity, watts/meter.

$Q=$ Flow, cubic ft. per min.

$U=$ Flow velocity, $\mathrm{ft} . / \mathrm{min}$.

$\eta_{A}=$ Efficiency of noise production

$=\frac{\text { noise power from fan }}{\text { Power to fan. }}$

$\eta_{s}=$ Static efficiency of the fan

$=\frac{\text { Air Power (based on } h_{s} \text { ) }}{\text { Power to fan. }}$

$\eta_{T}=$ Total efficiency of the fan

$=\frac{\text { Air Power (based on } h_{t} \text { ) }}{\text { Power to fan. }}$

$h_{t}=$ static head plus velocity head, inches of water. 


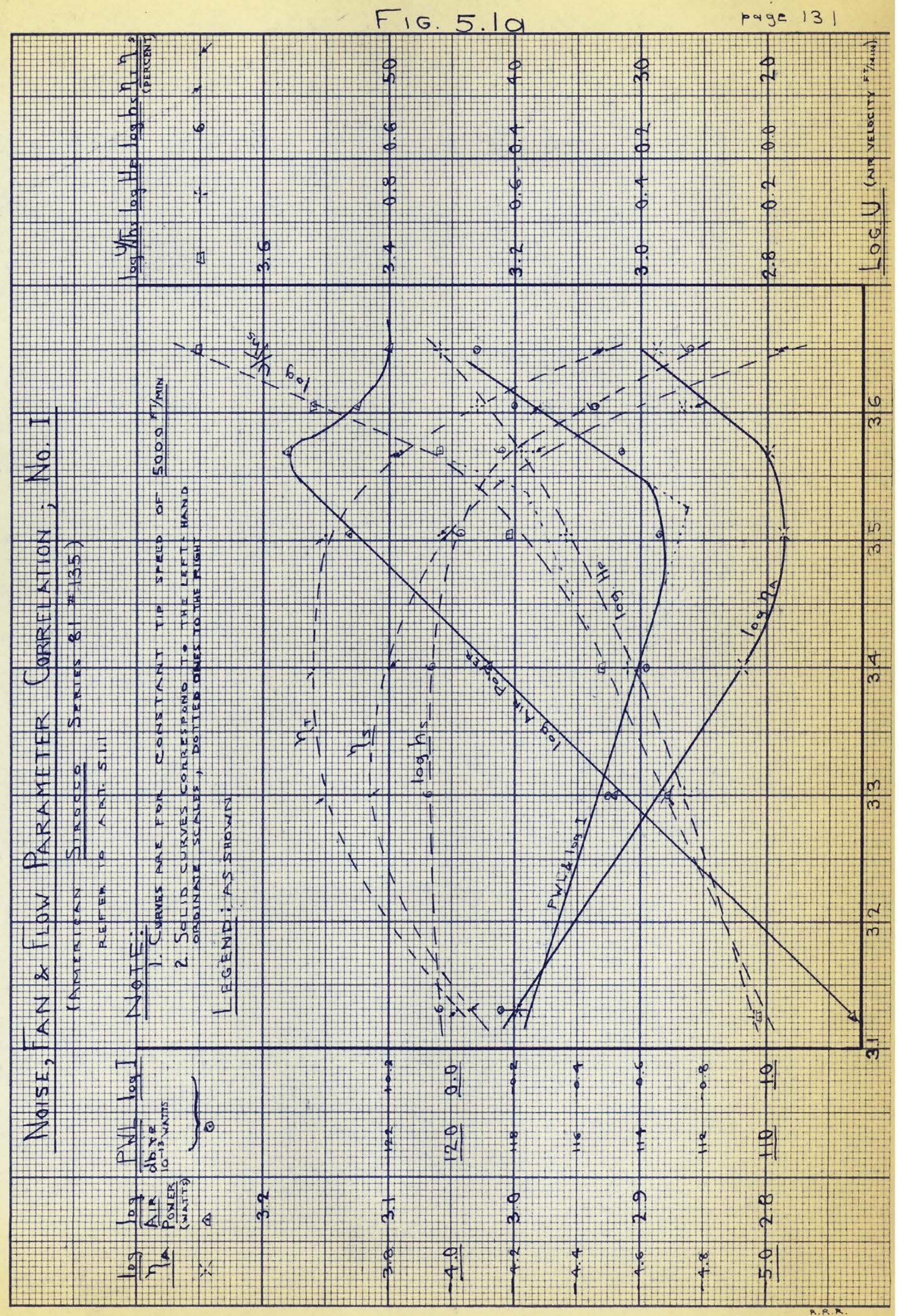




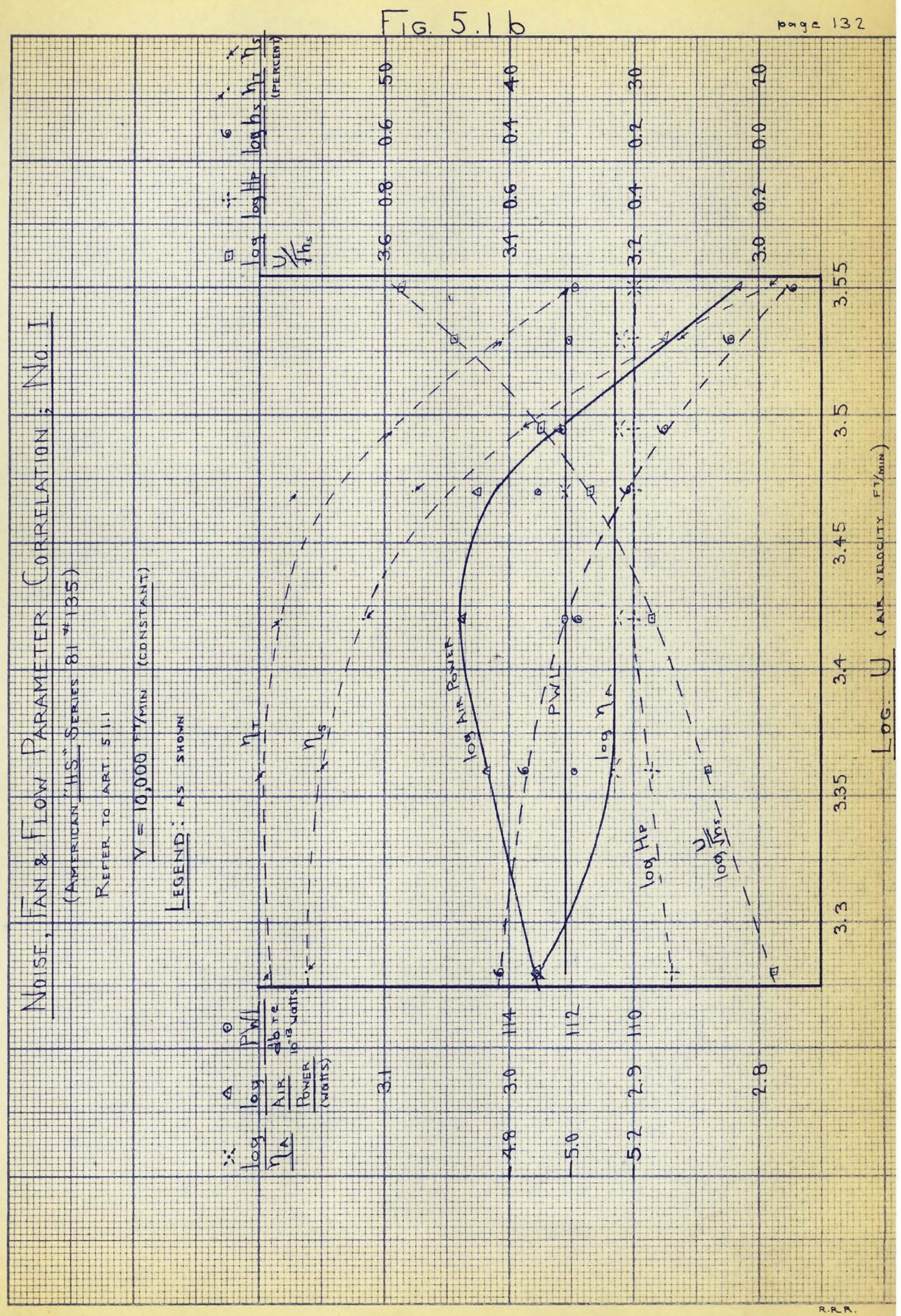


Comparing the PWL curve with the others:

1. The PWL - U curve has a minimum for the sirocco fan, but is nearly constant for the HS fan. The blade tip speeds are respectively $5000 \mathrm{ft} / \mathrm{min}$ and $10,000 \mathrm{ft} / \mathrm{min}$, corresponding to about the mid point of the useful speed range for these fans.

2. In the Sirocco case (Fig.5.1(a), the PWL minimum coincides almost exactly with the point where the unit capacity curve $U / \sqrt{h s}$ begins to change slope. This is further illustrated in Fig.5.2 for three different fan speeds.

3. The PWL minimum for the Sirocco fan coincides approximately with the maximum total fan efficiency and maximum Air Power.

4. In Fig. 5.1a, I decreases very nearly at the same rate as Air Power increases, below the minimum PWL.

5. The minimum of PWL for the Sirocco fan corresponds roughly with the constant PWL for the HS fan. (At these speeds, the two fans are operating nearly equivalently).

6. The efficiency of noise production $\eta_{A}$ displays a shape similar to the PWL curve and for both fans has a minimum near 10-5. The air velocity here is ab_out $3200 \mathrm{ft} / \mathrm{min}$, or in terms of Mach number $U / C$ is about 0.047 . According to Curle 34 (see also Theory, p.79 Art. 2.2), the efficiency of sound power production due to boundary layer turbulence is $\alpha(\text { Mach })^{3}$ for Mach $<l$. Using the above numerical value Mach $=0.047, \eta_{A}=10^{-4}$. Lighthill's quadrupole field due to air turbulence alone has an efficiency that increases approximately as $(\mathrm{Mach})^{5}$. Using $\mathrm{Mach}=0.047, \eta_{A} \cong 0.2 \times 10^{-6}$. This is evidence that the measured noise may be of an "aerodynamic" origin, primarily due to the action of solid 
Fig 5.2

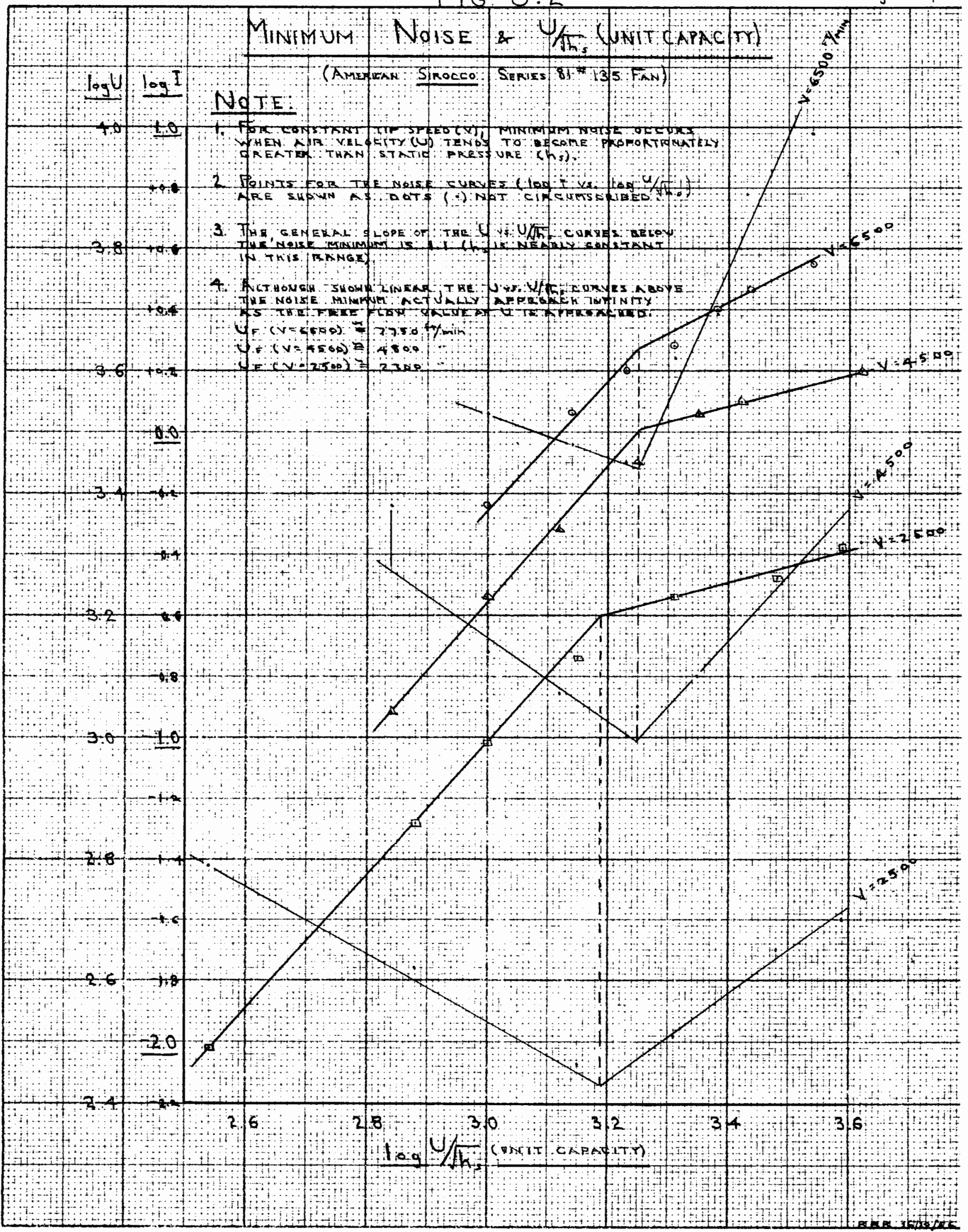


boundaries with air turbulence.

7. Although both fans appear equally noisy at the PWL min. for the sirocco fan, the Sirocco fan becomes noisier as the operating condition draws away from this point, but the HS fan does not.

From this preliminary set of results we can conclude that the Sirocco and HS fans appear not to follow the same noise laws and that thereis possible correlation with unit capacity, air power and total fan efficiency.

\subsubsection{SPECIFIC SOUND, SPECIFIC SPEED}

On page 16 of the literature review, drt. 1.1, mention was made of the concept of specific sound and specific speed of a fan. Specific speed is a well known concept of fan engineering whereby a homologous series of fans can have their operating characteristics defined by one universal curve of static efficiency vs. specific speed ( $\eta_{s}$ vs. $\left.N\right)$. This is true provided one is not considering operating conditions too remote from optimum (eg. maximum efficiency), as witnessed by $\eta_{s}-N s$ curves 3, 4, 5, Fig. 5.3a and 3, 4, Fig. 5.3b (refer to the legends for the operating condition these curves represent). The idea of specific sound, it was stated, originated with an unknown author contributing to Ref. 5\%. The subject matter concerning specific sound in this reference was related in Art.1.1. The idea is good, but requires further exploitation. To this end, two further specific sound concepts are defined: 


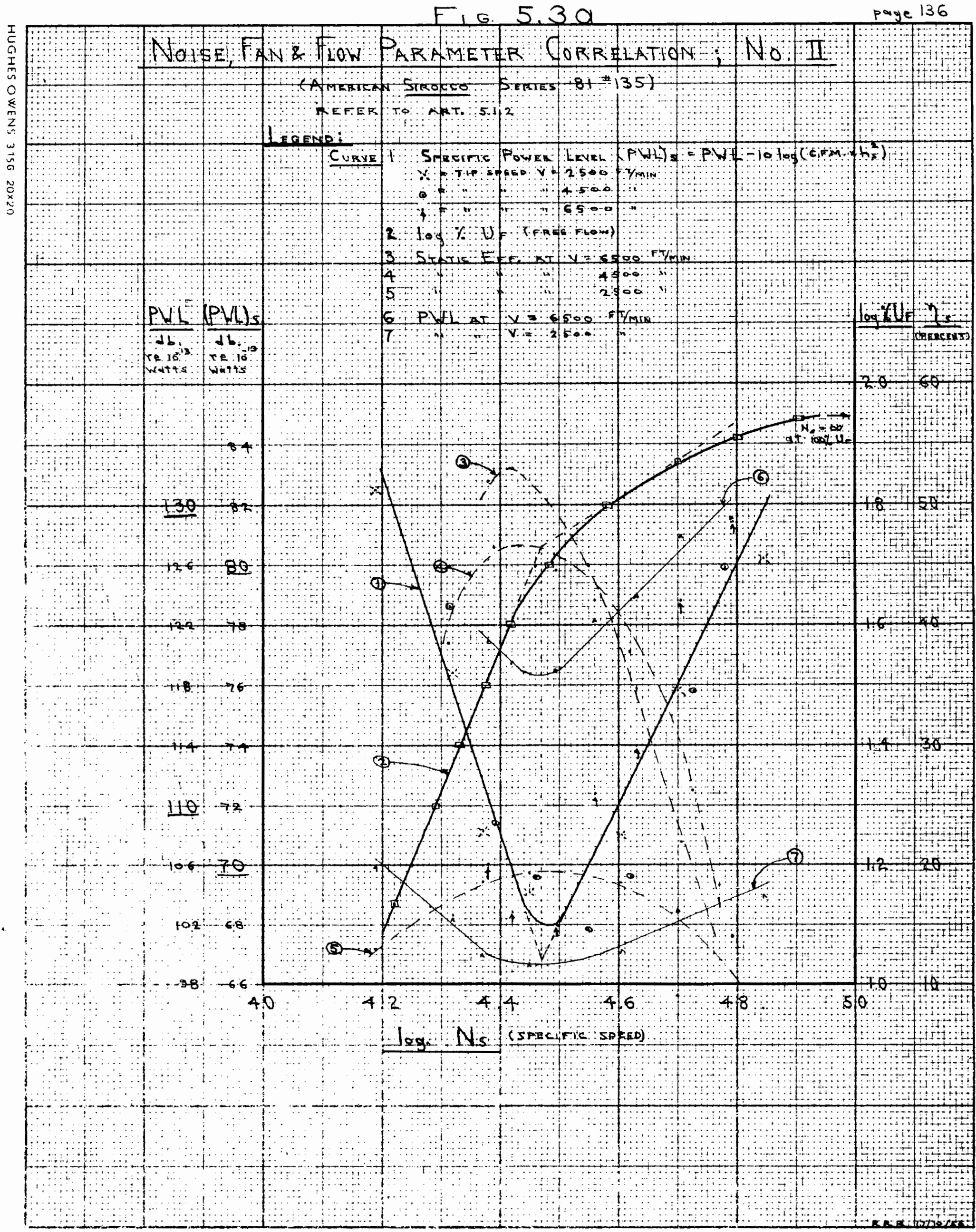


Fis $5.3 b$

page 137

$\frac{\text { Noise, Fan \& Flon Parameter Correlation; No II }}{\text { (Ammerican HS Serine } 81 \text { \% } 135 \text { Fans) }}$

$$
\text { TREFER TO NRT. 5.1.2. }
$$

\section{Leasnio:}

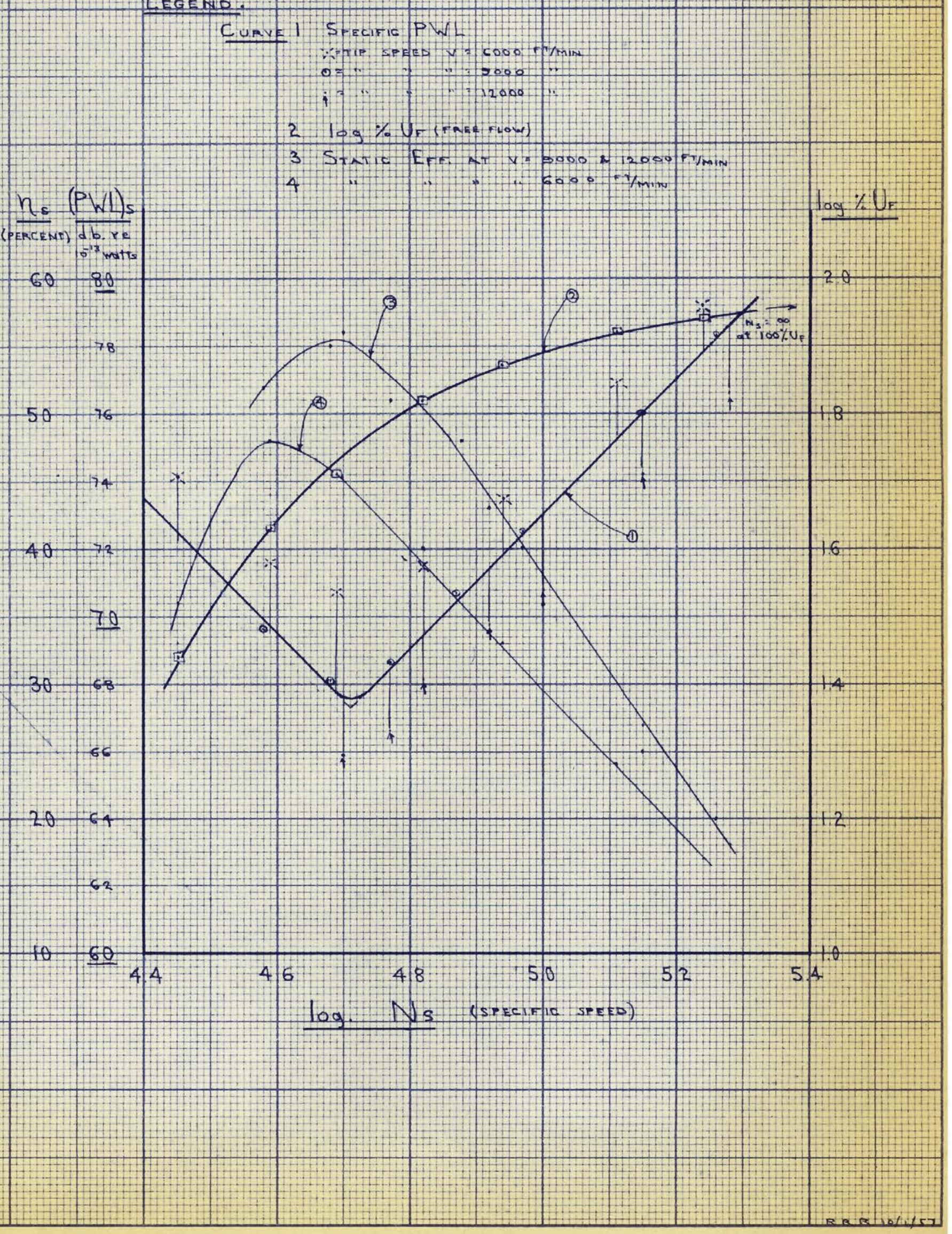


Specific Power Level

$(P W L)_{s}=P W L-10 \log \left(Q_{s}{ }^{2}\right) \quad \mathrm{db}$ re $10^{-13}$ watts $\ldots \ldots . .5 .1$ where PWL is the measured fan output noise power level. Specific Intensity

$(I)_{s}=\frac{10^{-13}(10)^{\frac{(P W L)}{10}}}{s}$ watts $/$ meter2 $\ldots \ldots \ldots \ldots \ldots \ldots \ldots .2$

10-13 is the reference level for PWLand (PWL) in watts.

$S=\begin{aligned} & \text { Duct area in which the noise intensity is being measured } \\ & \text { (meters2) }\end{aligned}$ Although the dependent sound variable is PWL or (PWL)s in the ensuing results, the variations of sound with various fan - flow parameters is more understandably expressed as the change in sound intensity $I$ and $(I)_{s}$ in a given duct as a function of the fan - flow variables.

(PWL)s vs. Ns is plotted for the sirocco fan as curve 1, Fig. 5.3a and for the HS fan as curve 1, Fig. 5.3b. Also plotted is the unit capacity curve (curve 2, both figures) and PWL - Ns curves for two speeds of the Sirocco fan, Fig. 5.2a curves 6 and 7. Data composing these results is for three widely separate fan speeds and for each of the seven Fsyst values (back panel adjustment) mentioned in Art.4. For at least a 2 to 1 fan speed ratio, comprising the useful portion of the fan operation, and a wide range of operating back pressures, the computed data (eq.5.1) forms a well defined $V$ curve (within $\pm 3 \mathrm{db}$ ) for each fan type (curve 1, Fig. 5.3a,b). Note that this single curve contains results from all the fan operating points over a range extended well above and below its optimum rating. Observe the following:

1. The (PWL)s - Ns curves contain all the information one 
need know about the overall noise power output of a given fan. By applying Eq. 5.1, the overall PWL of a given fan for any operating condition can be predicted.

2. The (PWL) $)_{s}$ Ns curves form a specific $V$ shape of straight sides. By the linear conversion for a given ducted fan condition from (PWL) to (I)s, Eq. 5.2, the slopes of the curve branches are as follows:

$$
\begin{aligned}
& \text { Sirocco: } \frac{\Delta(I)_{s}}{\Delta N_{s}}=-6 \text { below }(P W L)_{s} \min \text {. } \\
& =4 \text { above (PWL) min. } \\
& \text { HS: } \quad \frac{\Delta(I)_{s}}{\Delta N s}=-2 \text { below (PWL) } \min \text {. } \\
& =2 \text { above (PWLi)s min. }
\end{aligned}
$$

3. The (PWL) minimum occurs approximately at maximum $\eta$, for the highest speed and approximately at the point where the unit capacity - Ns curve deviates from linearity. 4. The (PWL)s minimum for the sirocco and HS runner are both about $68 \mathrm{db}$. Since both fans operate at almost the same $\mathrm{Q}$ and hs at this point, they are equally noisy. The Sirocco quickly becomes the noisiest away from this point of operation. This was found in Art. 5.1.1.

It appears very possible that for each homologous series of fans, the overall noise power level can be represented by a single typical curve of (PWL) $)_{s}$ vs. Ns. In other words, PWL data obtained from curve 1, Fig. 5.3a, b should coincide with measured date (within a reasonable margin of say $\pm 6 \mathrm{db}$ ) for all American Sirocco and American HS Series 81, single inlet fans. This represents a diameter range of $12^{\prime \prime}$ to 132 " and a capacity range of zero to 400,000 c.f.m. at 6" water gause. 
A striking comparison between the published hs - Ns curves for the Sirocco and HS fan types (Ref.54, p.246, curves $A$ and $D)$, and the $(P W L)_{5}$ - Ns curves found above is made in Fig. 5.4a,b(compare curves $I$ and 3 ). Two things are obvious: 1. The maximum of $\eta s$ - Ns and minimum of (PWL)s - Ns are nearly coincident.

2. The HS efficiency curve has a broader maximum than the Sirocco (this has been somewhat exaggerated because of the different efficiency scale used). There may, be some relation between this and the slope of the (PWL) - Ns curve on either side of the minimum (i.e., the broader the efficiency curve, the lower the slope of the specific sound curve).

There seems to be little correlation between the slope of the efficiency curve and corresponding slope of the sound curve, although it is not unreasonable to expect such correlation. The slopes are as follows (found after converting the efficiency ordinate to a logarithmic scale and replotting):

$$
\begin{aligned}
& \text { Sirocco fan: } \frac{\Delta(I)_{s}}{\Delta N_{s}}=-6 \text { and } 4 \\
& \frac{\Delta \eta_{s}}{\Delta N_{s}}=0.65 \text { and }-1 \\
& \text { HS } f a n: \\
& \begin{array}{l}
\frac{\Delta(I)_{s}}{\Delta N_{s}}=-2 \text { and } 2 \\
\frac{\Delta \eta_{s}}{\Delta N_{s}}=1.25 \text { and }-1.5
\end{array}
\end{aligned}
$$

The first figure corresponds to the slope below the maximum efficiency, the second figure, above. It appears that the lower the slope of the efficiency curve, the higher that of the (PIVL)s curve, although there are no grounds for this. The opposite is what would be expected. 


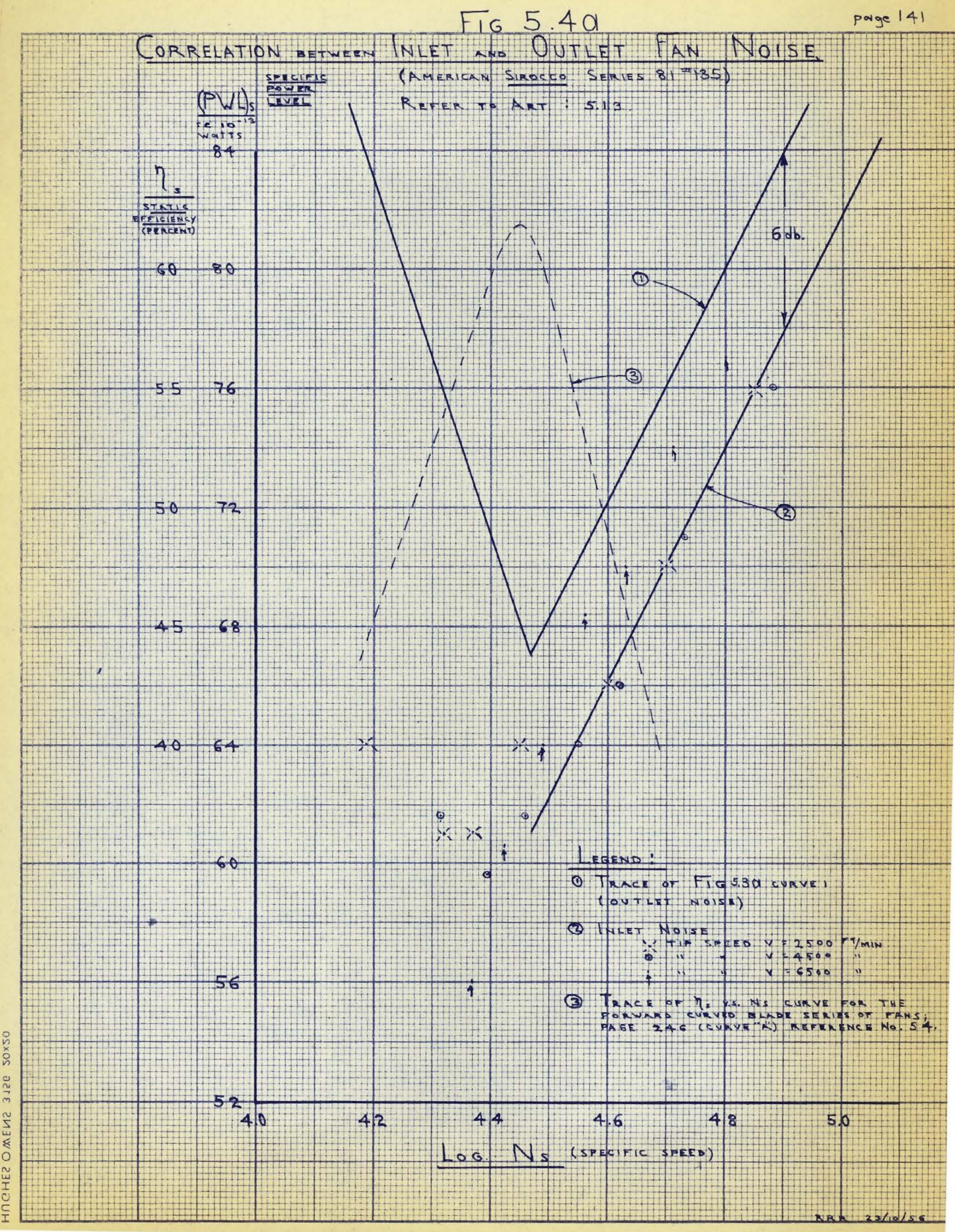




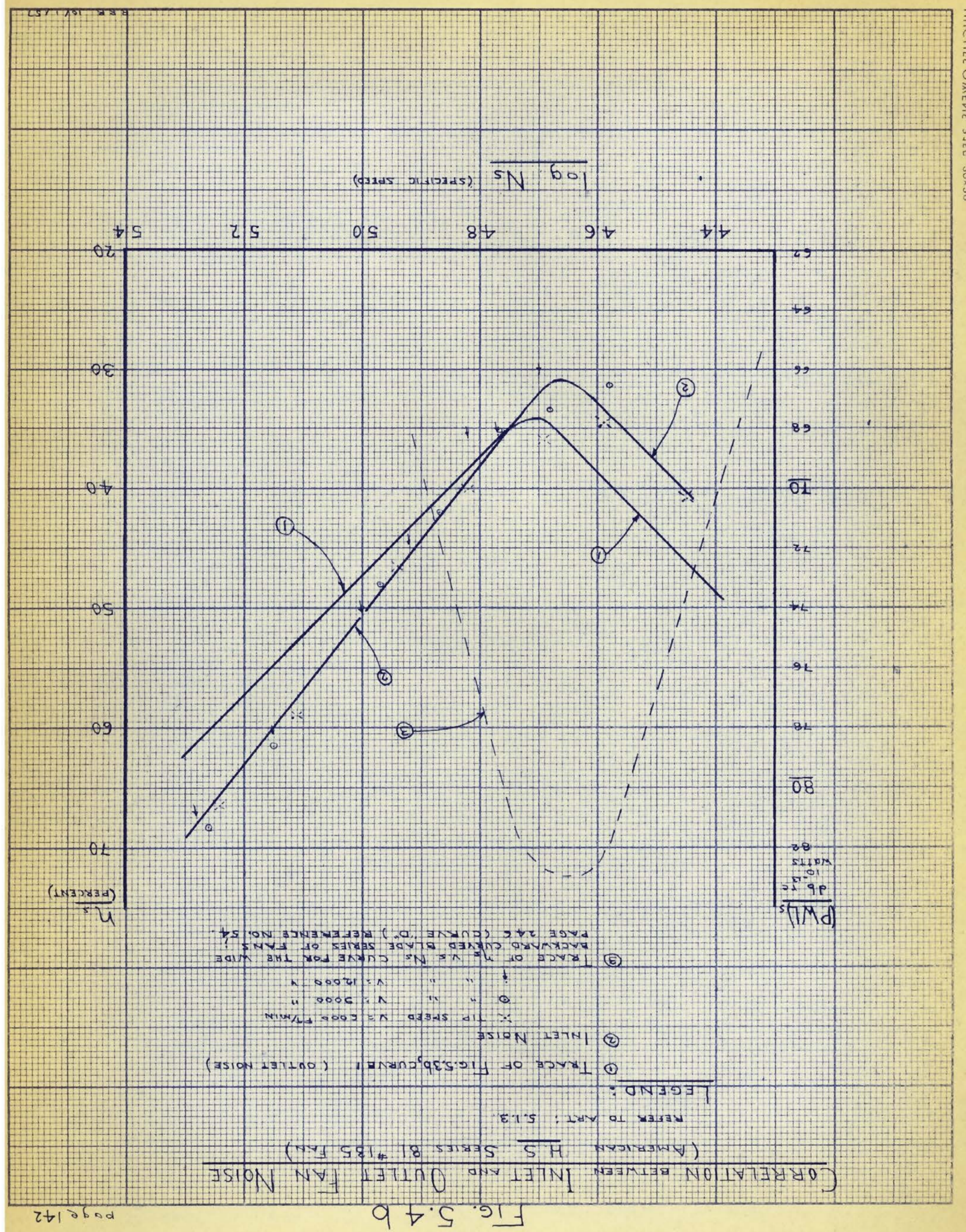


In concluding this section, the specific sound concept appears to be a most valuable tool for the estimation of overall fan noise. If testing of other fans in the same series gives almost identical curves, as they should, then armed with this one curve and applying eq. (5.1) one can predict the probable overall fan noise PWL for any size of $f$ an of a homologous series, for any operating condition. It will be iliustrated later that most of the fan noise power is of "boundary turbulence origin" (see pp.67,68 Art. 2.2 for the significance of this), hence it is reasonable to expect the (PWL)s - Ns curve to hold for other fan sizes in the same series since the scale of air flow involved increases proportionately and other noise such as vibration is small in comparison.

\section{1 .3 INLET VS. OUTLET NOISE}

All measurements for these curves were made at the microphone location shown in Fig. $1.3 \mathrm{p} .18$. The noise measured here is almost equivalent to the noise at the fan outlet, since the duct cross sectional area is approximately the same as the area of the fan outlet. It is often inconvenient to measure noise from installed fans at this location. Usually the inlet is readily accessible. It would be of some value to compare the overall noise levels at these locations to determine whether a correlation exists between them. It should since, as will be shown, much of the noise power is generated by boundary turbulence at the blades. The inlet station was chosen as the axis of the inlet eye, at the junction of the bell mouth outer face with the fan casing. At this point it is a short 
distance from the runner, and is just within the inlet flange. It is analogous to being just inside a short length of open duct. Due to mismatch between the inlet flange and "free space", the PWL will be somewhat higher just inside the flange than outside. At this position with the $23 / 4^{\prime \prime}$ windscreen intact, there was but small change of the air flow characteristics of the fan.

The results are shown in Fig. $5.4 a, b$, curve 2. These curves represent the same wide range of operating points that curve 1 in the same figures do. The correlation appears relatively good for specific speeds above the $\eta_{\text {s maximum. Data be- }}$ low this for the Sirocco fan showed no form, while for the HS fan did display useful correlation. The inlet noise for the Sirocco fan is displaced about $6 \mathrm{db}$ below the outlet noise while for the HS $f a n$, it is actually above the outlet noise for higher Ns. This is due in part to additional noise generated at the bell mouth of the HS fan because of venturi - like shape and higher inlet air speeds. More will be said of this in Art.5.3. The acoustic characteristics of the two inlets and runners differ, thus the degree of noise transmitted outward from the inlet will differ.

Curve 2, Fig. 5.4a, b maintains its slope, and decreases in magnitude as the microphone is moved axially away from the fan inlet. This held only out to about 6 inches from the inlet flange (12" from the sirocco runner) at which point the ambient noise of the motor, plus the reverberant nature of the room affected the overall noise level. The overall level dropped about $6 \mathrm{db}$ at $6 "$ compared to that measured above. When the 
microphone was inside the open inlet flange, the PWL $=$ SPL because the inlet area $=1 \mathrm{ft} .^{2}$. The SPL falls quite rapidly outside of the flange due to mismatch between flange and space and the diffusion of the noise energy over a wider area. For reliable measurements, the windscreen covered microphone should be just inside the inlet flange. The ambient motor noise for a given fan speed was found to be about $10 \mathrm{db}$ or more in each octave band below the measured inlet fan noise.

For operation near maximum efficiency, or above maximum efficiency, adding $6 \mathrm{db}$ to the inlet PWL for the Sirocco fan, and leaving the HS inlet PWL unchanged should represent roughly the overall PWL of fan outlet noise (when the outlet is ducted). A similar, though not identical, correlation should exist when the fan is used as an exhauster, with ducted inlet and free discharge.

\subsubsection{DUCTED CENTRIFUGAL FAN NOISE LAWS}

It appears probable from Fig. 5.1 and 5.2 that the vatiation of fan noise power with various fan and flow parameters will be quite different for different fan types. Not only that, but there seems to be a further division of laws for each fan type, differing from one side of the $\eta_{s}$ maximum (PWL minimum) to the other. It is very probable that each slope of the $(P W L)_{s}-\log$ Ns curve represents a different set of laws.

What follows now is a wide selection of curves representing nearly all the useful variations of fan PWL with flow fan parameters as independent variable. These results, Fig.5.5 
to 5.12 , were constructed mainly from the four basic experimental curves as outlined in Art. 4. The letter"a" following the figure number represents sirocco results, "b" - H.S fan results. These results are now briefly discussed. The reader is requested to refer to the glossary of symbols whenever necessary as most of these symbols have been used previously. Noise intensity I watts/meter ${ }^{2}$ is the dependent variable used in the laws.

Fig. 5.5. PWL - log. \% Ur' (free flow), speed constant.

A universal way of expressing fan discharge into a system offering a certain back pressure is as a fraction of the flow the fan would deliver into free space (back pressure, hs=0). For each constant speed selected, there is a specific U. (free flow air velocity). As back pressure increases, for constant speed, the actual flow velocity as a fraction of $U$ decreases. This then forms the abscissa of Fig. 5. Air velocity $U_{F}$ is the independent variable, modified to the form $\log \% \mathrm{U}_{\mathrm{F}}$.

PWL vs. log \% U.r at constant speed (eg. blade tip speed $V$ constant) is a well defined V-shaped curve for the Sirocco fan and a rather poorly defined shallow one for the HS fan. Their shape as a function of speed is relatively constant. From Fig. 5.1, the nearest correlation is with Air Power (which is proportional to $U \mathrm{x}$ hs). A given increase in speed does not yield a proportional increase in PWL, but something less than this. The behaviour of these constant speed curves is best described as follows: 


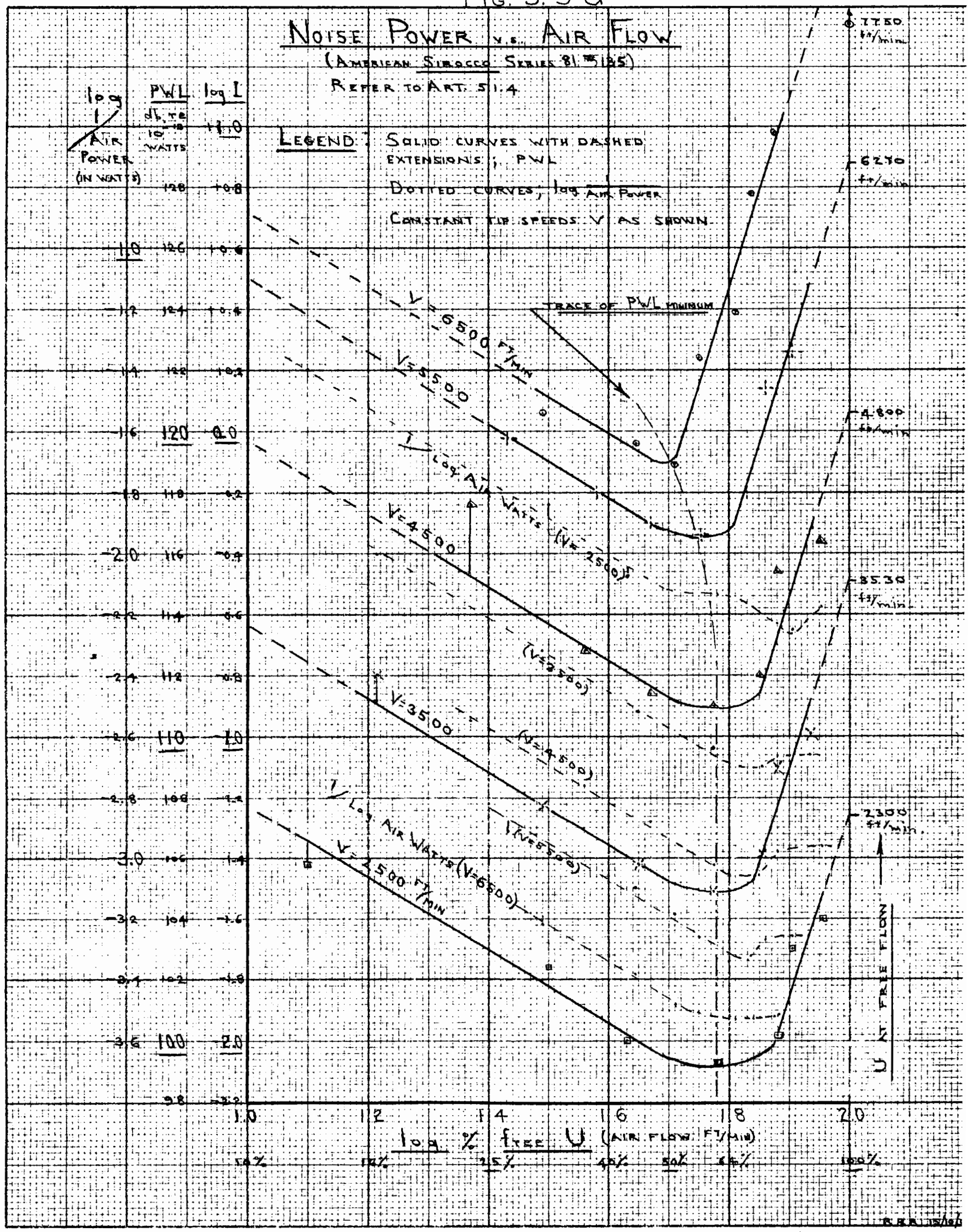




\section{Fo. $5.5 \mathrm{~b}$}

page 148

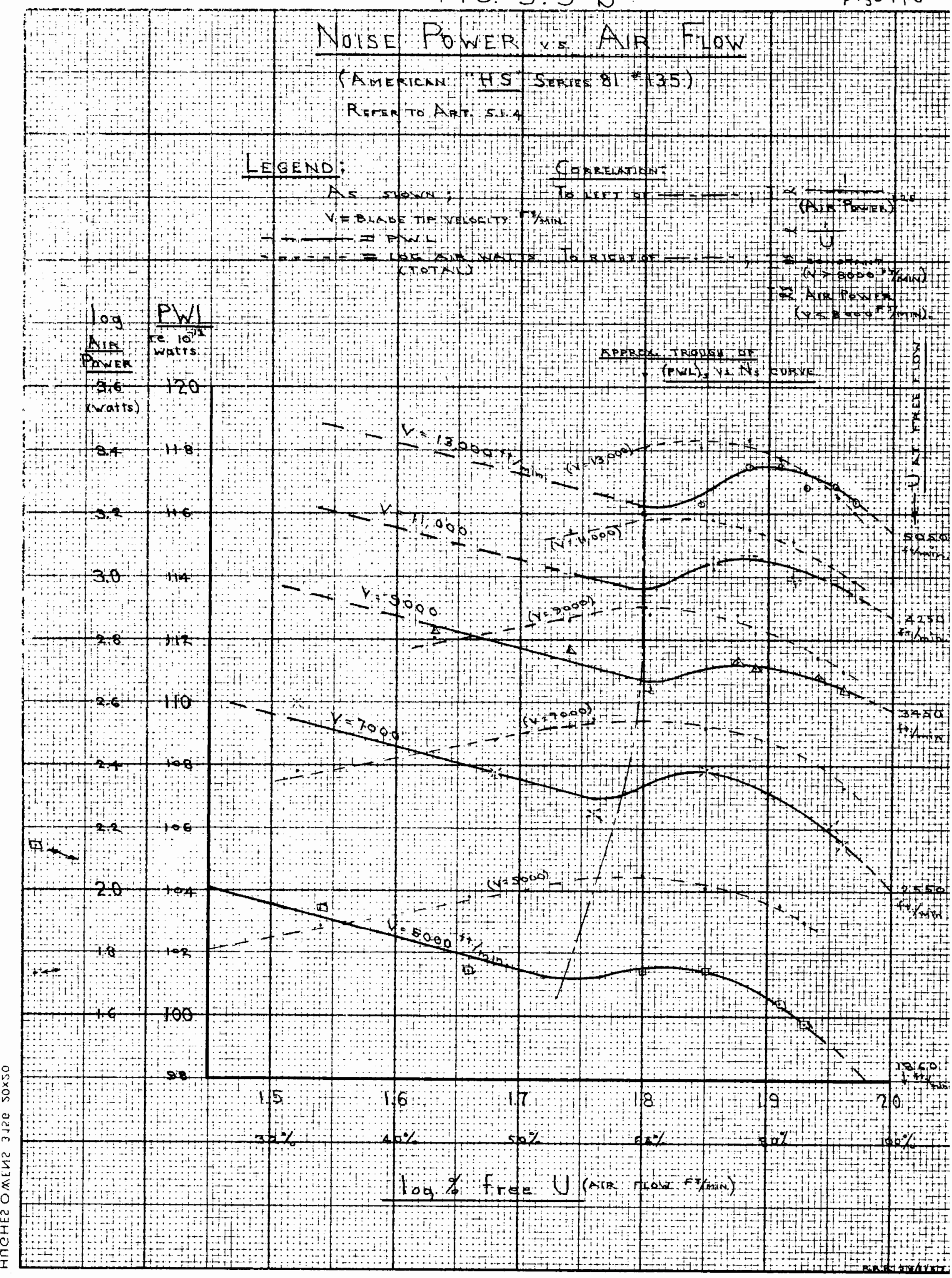




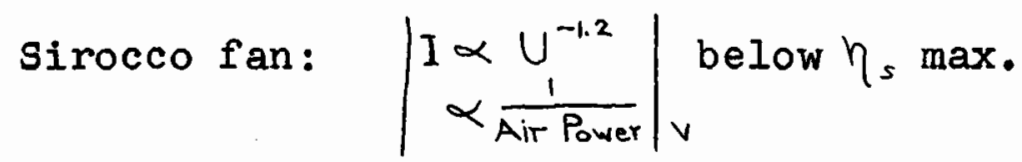

$$
\left|I \propto U^{6}\right|_{v} \text { above } \eta \text {, max. }
$$

HS fan:

$$
\begin{aligned}
& \left|I \propto U^{-1}\right|_{V} \text { below } \eta_{s} \max . \\
& \left.\begin{array}{l}
{[I=\text { Constant }]_{V} V>8000 \mathrm{ft} / \mathrm{min}} \\
{[I \times \text { Air Power }]_{V} V<8000 \mathrm{ft} / \mathrm{min}}
\end{array}\right\} \text { above. } \eta_{s} \max .
\end{aligned}
$$

The subscript ( $\mathrm{V}$ in this case) outside the brackets indicates the parameter held constant. The term $\eta_{s} \max$. refers to the maximum static efficiency of the fan as discussed in connection with Fig. 5.4. It corresponds approximately to the minimum fan noise. The laws above $\eta_{s} \max$, are to be taken to apply at $\eta_{s} \max$. also.

Fig. 5.6 PWL vs. log U (flow velocity), back pressure $\left(h_{s}\right)$ constant.

The independent variable here is simply the log. of flow velocity $U$. The significance of constant back pressure $h_{s}$ is that as $U$ increases the back panels for pressure regulation must be opened more and more. This follows because hs $\approx U^{2}$ and if the system were held constant, hs would increase with $U$. This set of curves has use only if one has a fan which is to be operated at a specific condition of hs, but variable $U$ and Fyst (meaning variable system as seen from the fan). The relations are:

Sirocco fan: $\left[I \propto U^{-2}\right]_{h s}$ below $\eta_{s} \max$.

$$
\left[I \propto U^{6}\right]_{h s} \text { above } \eta_{s} \max \text {. }
$$

HS fan: $\quad[\mathrm{I}=$ constant with $U]$ hs $>2.5^{\mathrm{N}} \mathrm{H}_{2} \mathrm{O}$

$$
\begin{aligned}
& {\left[I \approx U^{-0.5}\right] \text { hs }<2.5^{\prime \prime} \mathrm{H}_{2} \mathrm{O} \text { below } \eta_{s} \max .} \\
& {\left[I \propto U^{2.5}\right]_{h_{s}} \text { above } \eta_{s} \max .}
\end{aligned}
$$


Fig.5.6a

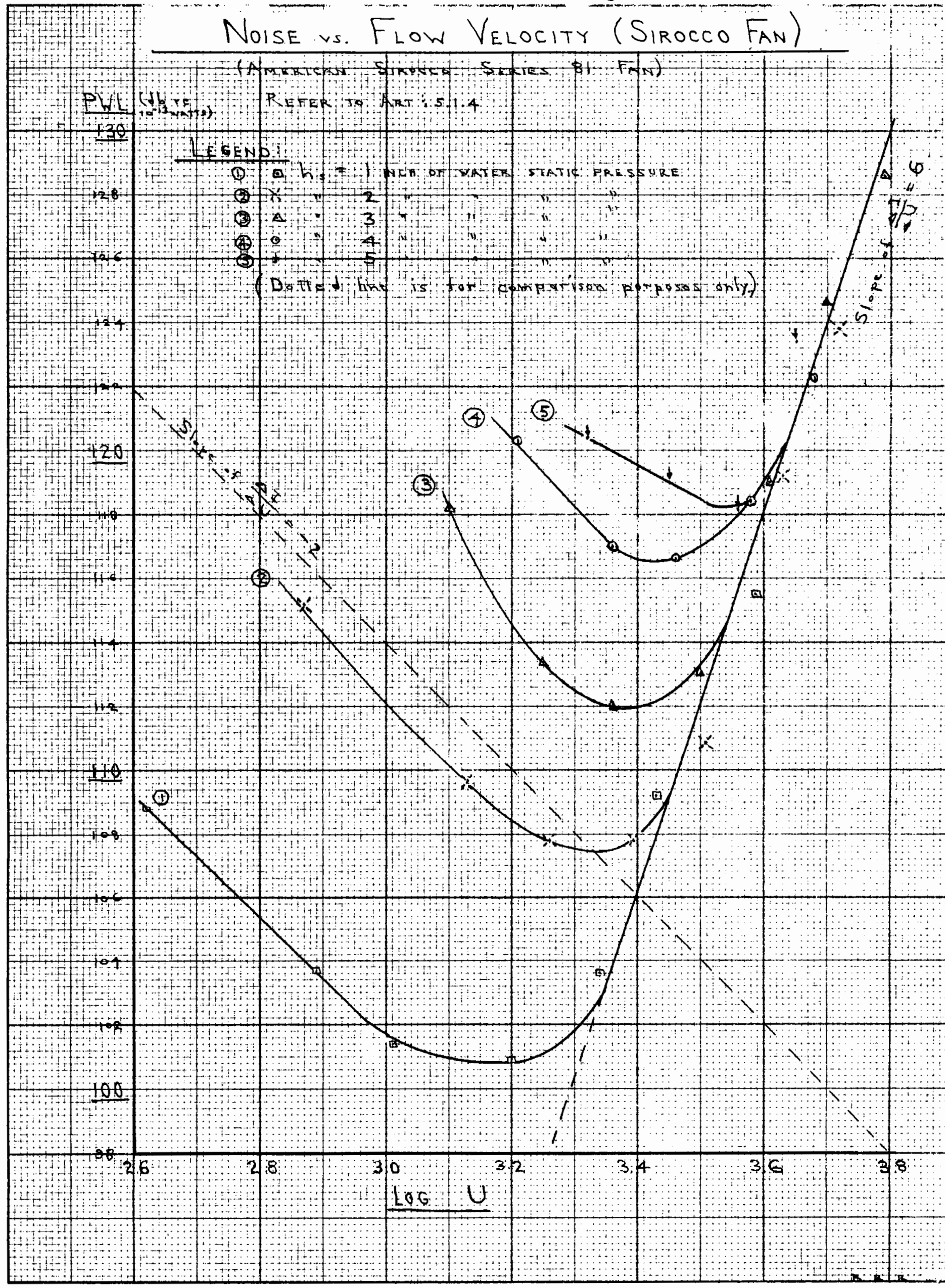




\section{Fig. $5.6 \mathrm{~b}$}

pare 151

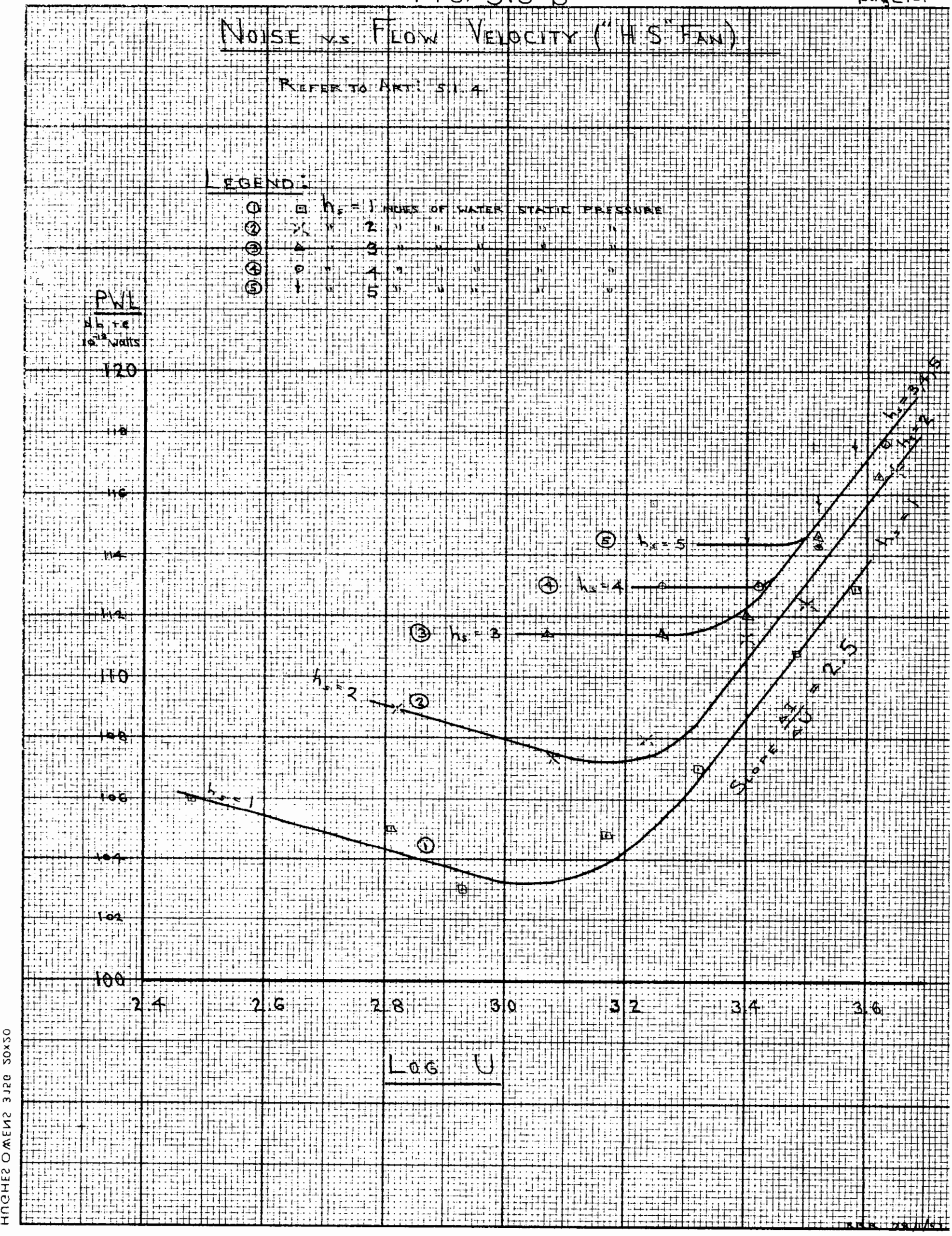


Fig. 5.7 PWL vs, log. back pressure (hs), flow constant.

A common situation in ventilating problems is that the air volume required is the first known, and all the other unknowns follow in the system design and fan selection. For a given capacity, or air velocity in the main duct, the back pressure the fan must operate against is a function of the ventilating system inserted between the fan and room to be ventilated. It is instructive to know how the noise level will vary with the back pressure (system design). Fig. 5.7a, b display similar patterns of variation:

Sirocco fan: $[I=$ constant with hs $]$ u below $\eta_{s} \max$.

$$
\left[I \propto h s^{2.5}\right] \cup
$$

HS fan:

$$
\begin{array}{ll}
{\left[I \propto h_{s}^{0.5}\right]_{u}} & \text { below } \eta_{s} \max . \\
{\left[I \propto h_{s}{ }^{2}\right]_{U}} & \text { above } \eta_{s} \max .
\end{array}
$$

This ends the relations of constant parameter other than the ventilating system itself ( $F_{\text {syst }}=$ constant $)$. Before turning to these, it should be pointed out that there are various additional curve families involving $\mathrm{Hp}$ and speed as the constant parameter or as independent variable. These two variables are only considered with Fsyst constant. Hp and speed have not been plotted with $U$ and hs, because these latter are the more fundamental in any ventilating system. Hp and speed follow naturaliy once $U$ and hs are known. $U$ and fan speed are generally proportional for a given fan, hence they can be interchanged in the graphs. If one desires relations other than those presented here, they can be computed from the (PWL) -Ns $^{-N}$ curve for the fan type in question, and Eq. 5.1. All data plotted here are of course measured values. We now turn to relations 
Fig. $5.7 \mathrm{a}$

porge 153

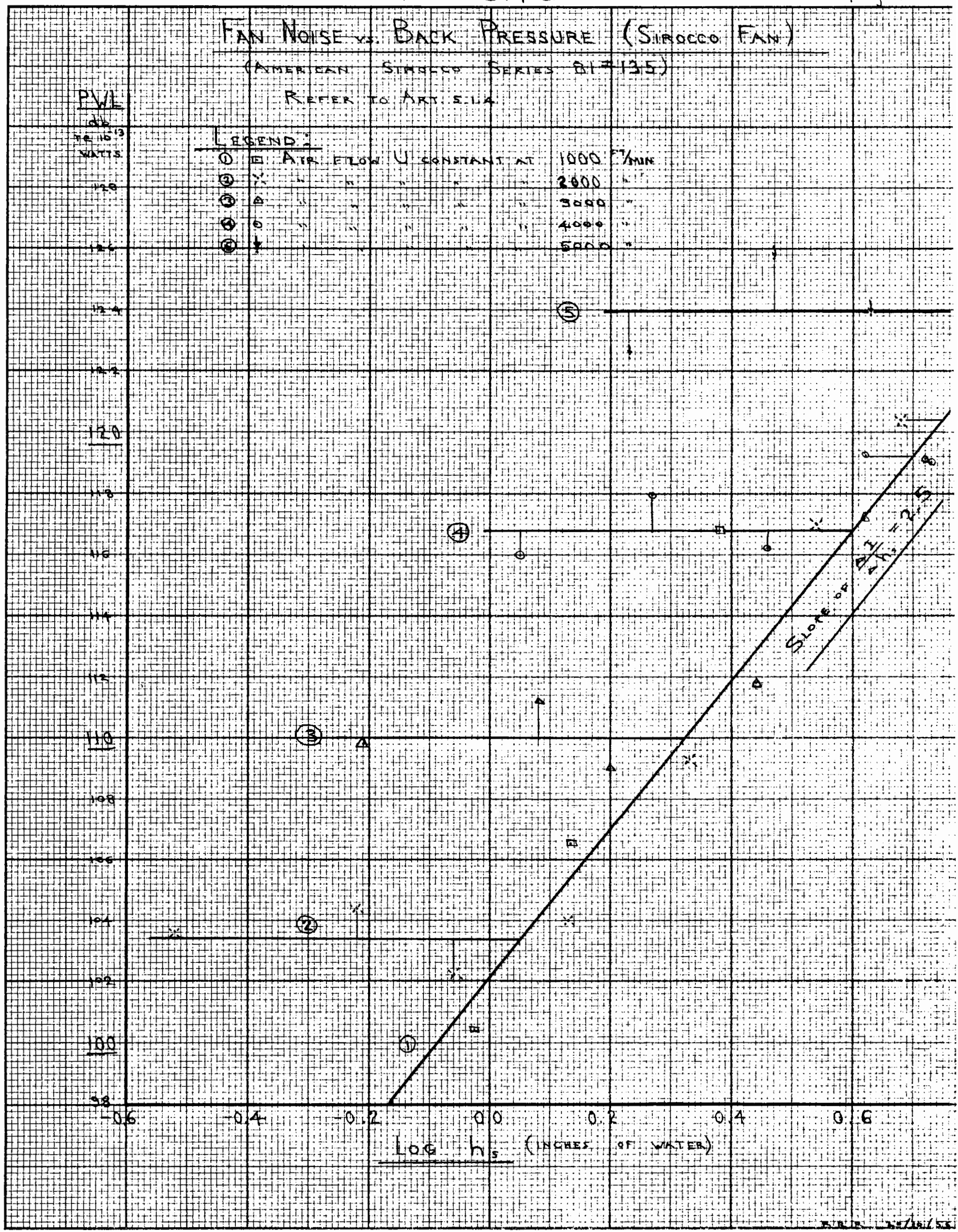


Fig $5.7 \mathrm{~b}$

paye 154

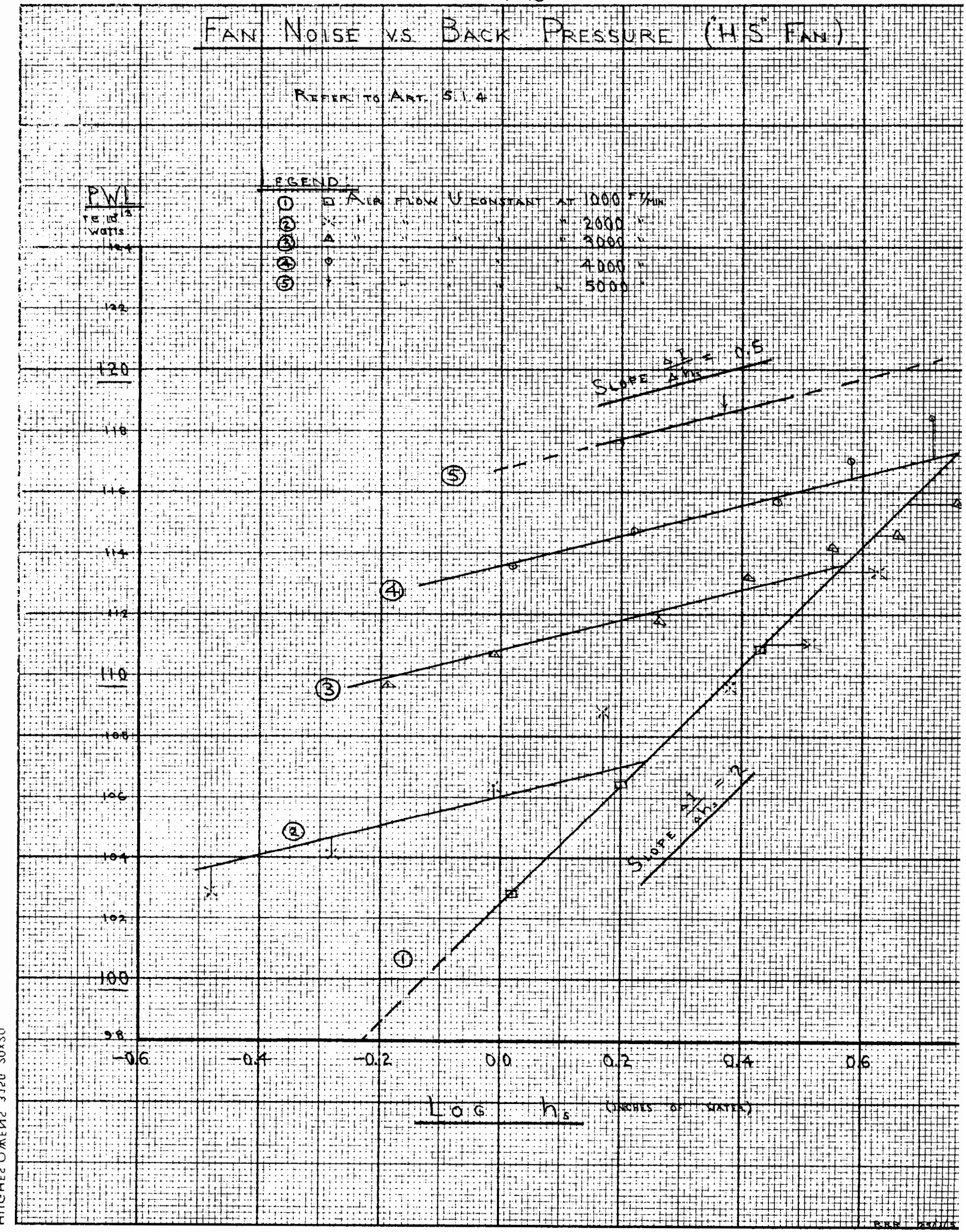


of PWL, (PWL)s, U, hs, V, Hp with $F_{\text {syst }}$ as a constant parameter. This is the parameter which has been assumed held constant in all the fan "noise laws" given in the literature review Art. 1.1 .

Fig. 5.8 PWL vs. $\log$ blade tip velocity ( $\mathrm{ft} / \mathrm{min})$, Fyst constant.

The commonly held view in North America is that fan noise intensity varies as the 5 th power of the fan speed. In a British handbook 55 it varies as the 5.5 power of fan speed. In part, both are correct, but not for all operating conditions or fan types. In Art. 2.2 it was inferred that the boundary turbulence noise from a rotating system could vary from a 4 th power of speed to a power of 7 , depending on the details of the rotating system and airflow. The results of Fig. $5.8 \mathrm{a}$ and $\mathrm{b}$ show a dependance on speed running the gamut from 1 to 8 , depending on the fan type and operating condition. Operation near free flow (F $F_{\text {syst }}$ low) however does show a more or less uniform dependence of $I$ on the 5 th power of speed.

In Fig. 5.8, three curves have been plotted, corresponding to three positions of the back panels (seven curves in all were taken). Curve $I$ is typical for $F_{\text {syst }}$ low. This corresponds to high air flow, low back pressure operation with specific speed (Ns) well above the $\eta$ s max. point. Curve 2 is for $F_{\text {syst }}$ medium, corresponding to the $\eta_{s} \max$. (PWL min.) point. Curve 3 is for high hs and $\mathrm{Ns}$ well below the $\eta$ max. point. Attention should be paid only to the solid curves in the upper half of Fig. $5.8 \mathrm{a}, \mathrm{b}$. The solid curves in the lower half show the corresponding increase of back pressure with fan speed for each of the above mentioned $F_{\text {syst }}$. We have the rather incon- 


\section{Fig. 5.80}

page 156

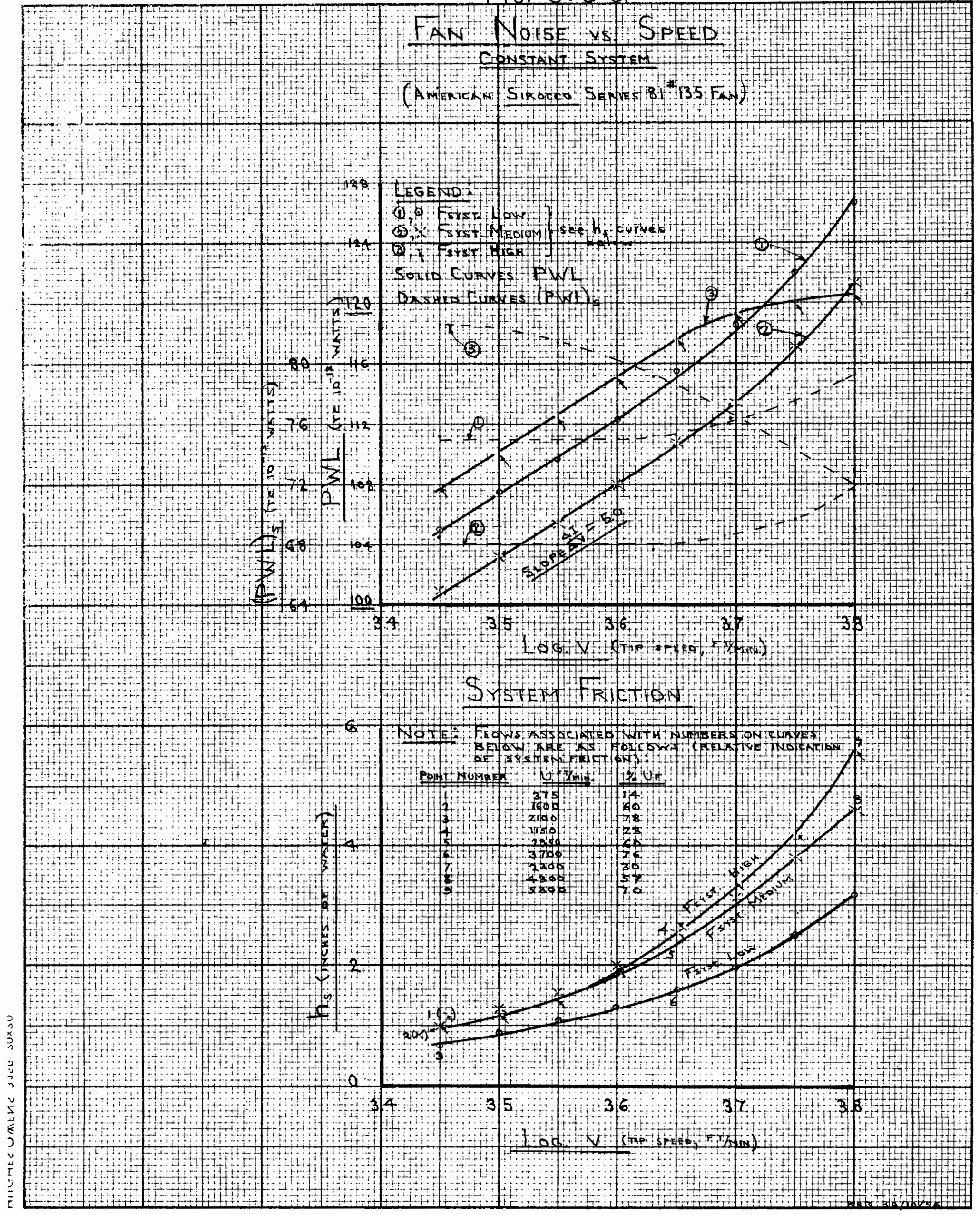




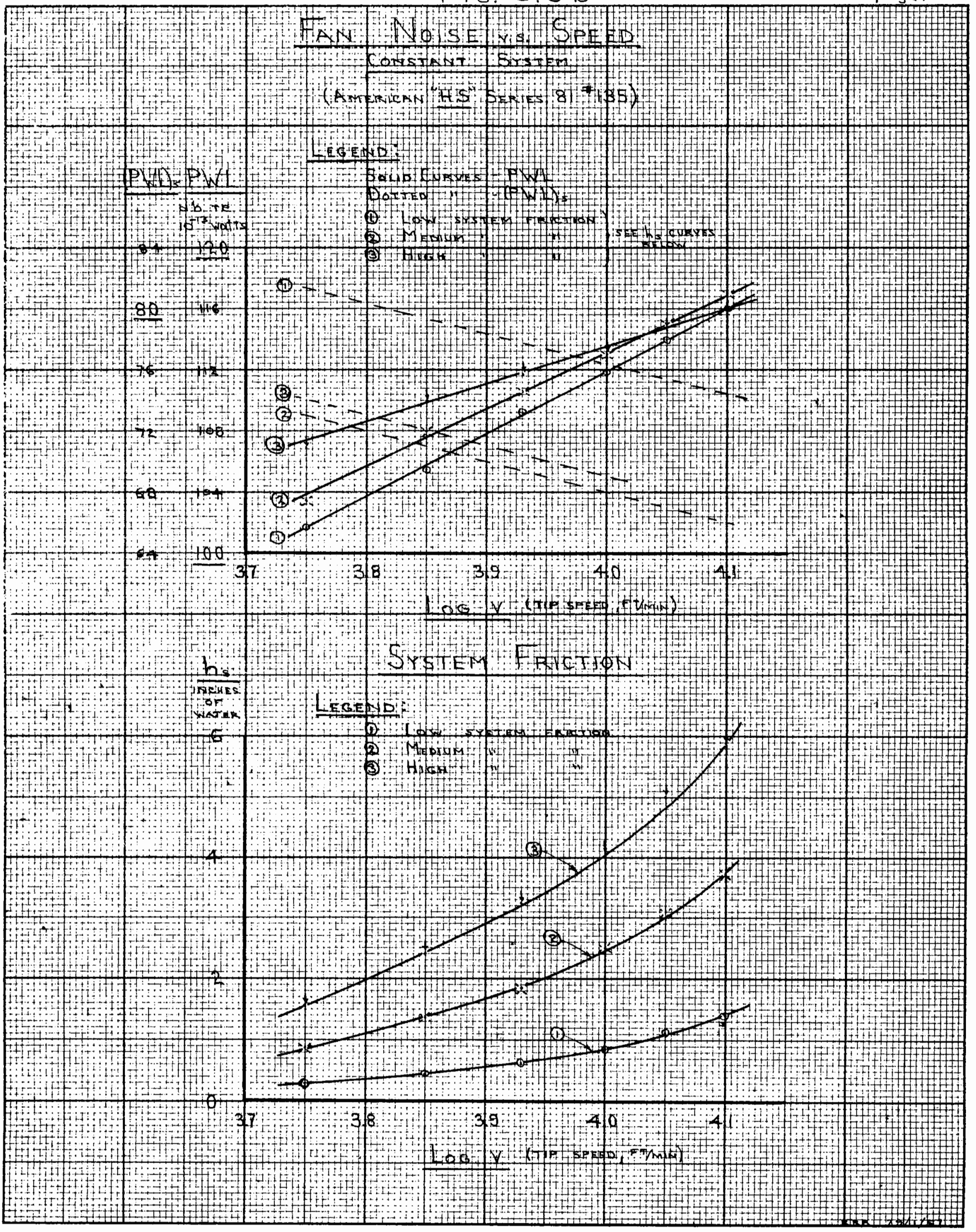


sistent relations:

Sirocco fan: $\left[I \propto V^{5}\right] \mathrm{F}_{\text {syst.; }}$ for $\mathrm{V}<4500 \mathrm{ft} / \mathrm{min}$

$$
\begin{aligned}
& {\left[I \propto V^{5 t o 8}\right] \begin{array}{l}
\text { Felow and above } \eta_{s} \text { max. } \\
\text { above } \eta \text { for } V>4500 \mathrm{ft} / \mathrm{min}
\end{array}} \\
& {\left[I \propto V^{t+5}\right] \text { Fsyst; for } V>4500 \mathrm{ft} / \mathrm{min}} \\
& \text { below } \eta_{\text {s max. }}
\end{aligned}
$$

HS fan: $\quad\left[I \propto V^{2 t_{0} s}\right]$ Fsyst; where the larger exponents are for operation above $\eta$, max., the smaller exponents below.

In conclusion, the variation of PWL with fan speed is not a dependably constant variation, hence should be used with caution.

A better relation appears to be specific sound vs. V for the HS fan, but is of little added value for the sirocco fan. (PWL) $)_{s}-\log V$ curves are represented by the dashed lines in the upper half of Fig. $5.8 \mathrm{a}, \mathrm{b}$. We see for the HS fan they are a uniform family so that

$\left[(I)_{s}\right.$ (eg.specific intensity) $\left.\propto V^{-2}\right] F_{\text {syst; }}$ above and below $\eta_{s} \max$.

Fig. 5.9 PWL vs. $\log U$ and $\log$ hs, $F_{\text {syst constant }}$

The upper half of Fig. $5.9 \mathrm{a}, \mathrm{b}$ contains two sets of curves. The solid ones are PWL vs. $\log U$ for the same three $F_{\text {syst }}$ as for Fig. 5.8. The dotted ones are the corresponding variations of hs with each of the $F_{\text {syst. We find a dependency }}$ of $\Delta I$ vs. $\Delta U$ of from 1.25 to 6 , which is not surprising since there is a close relation between $V$ and $U$ and $\Delta I$ varied with the first to the eighth power of $\mathrm{V}$ in Fig. $5.8 \mathrm{a}, \mathrm{b}$. The dotted extension of solid curve 3, Fig. 5.9a is a rough indication of 


\section{(14)}

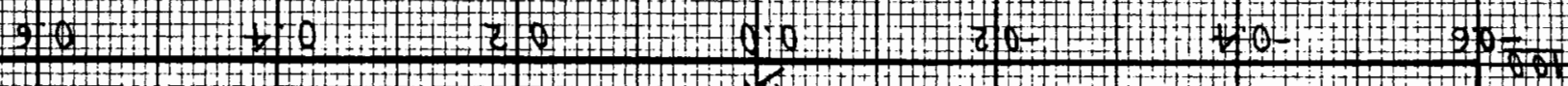
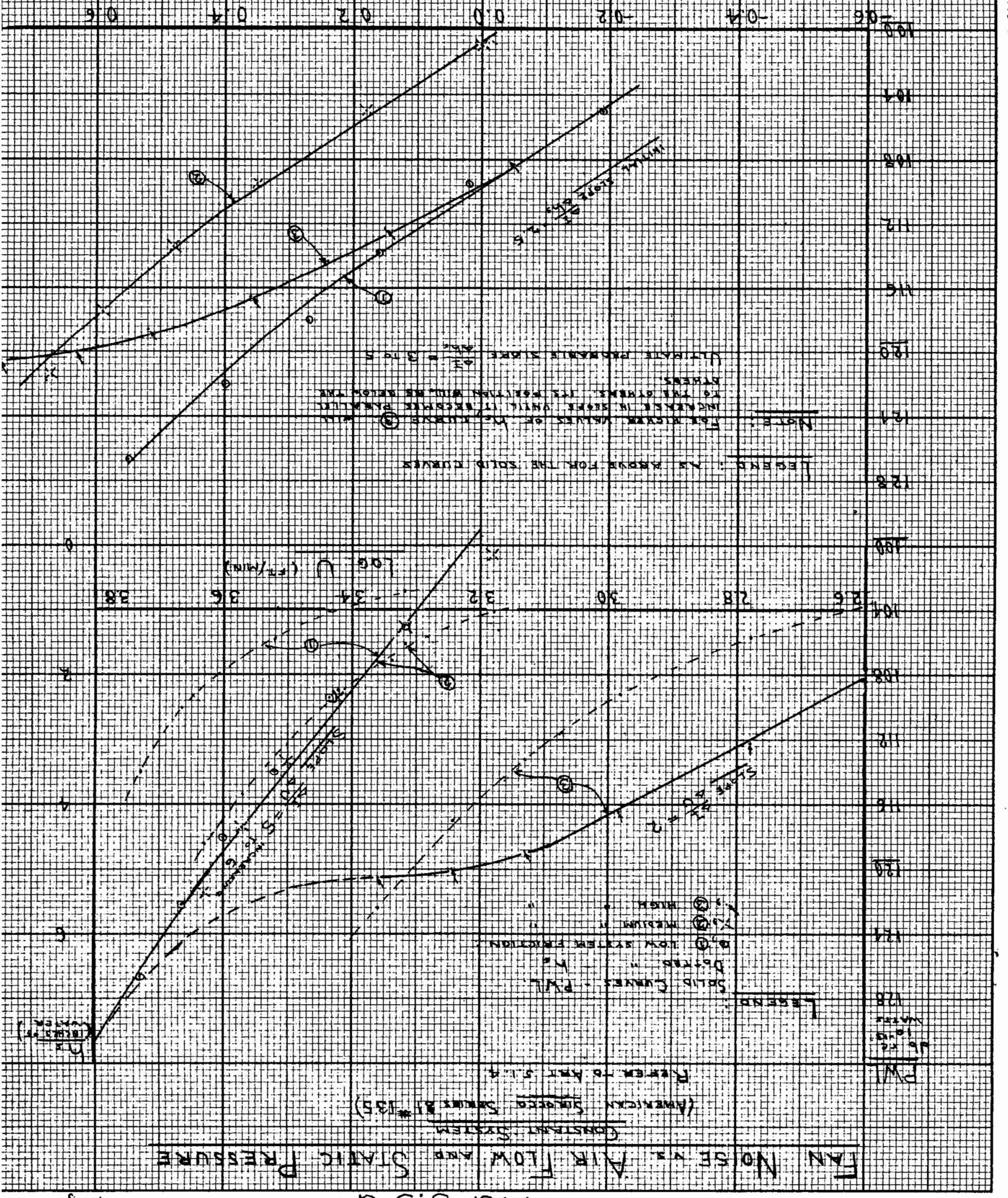

$6 s 1^{26} b_{0} d$

$$
10.9 \cdot 9 ! 7
$$




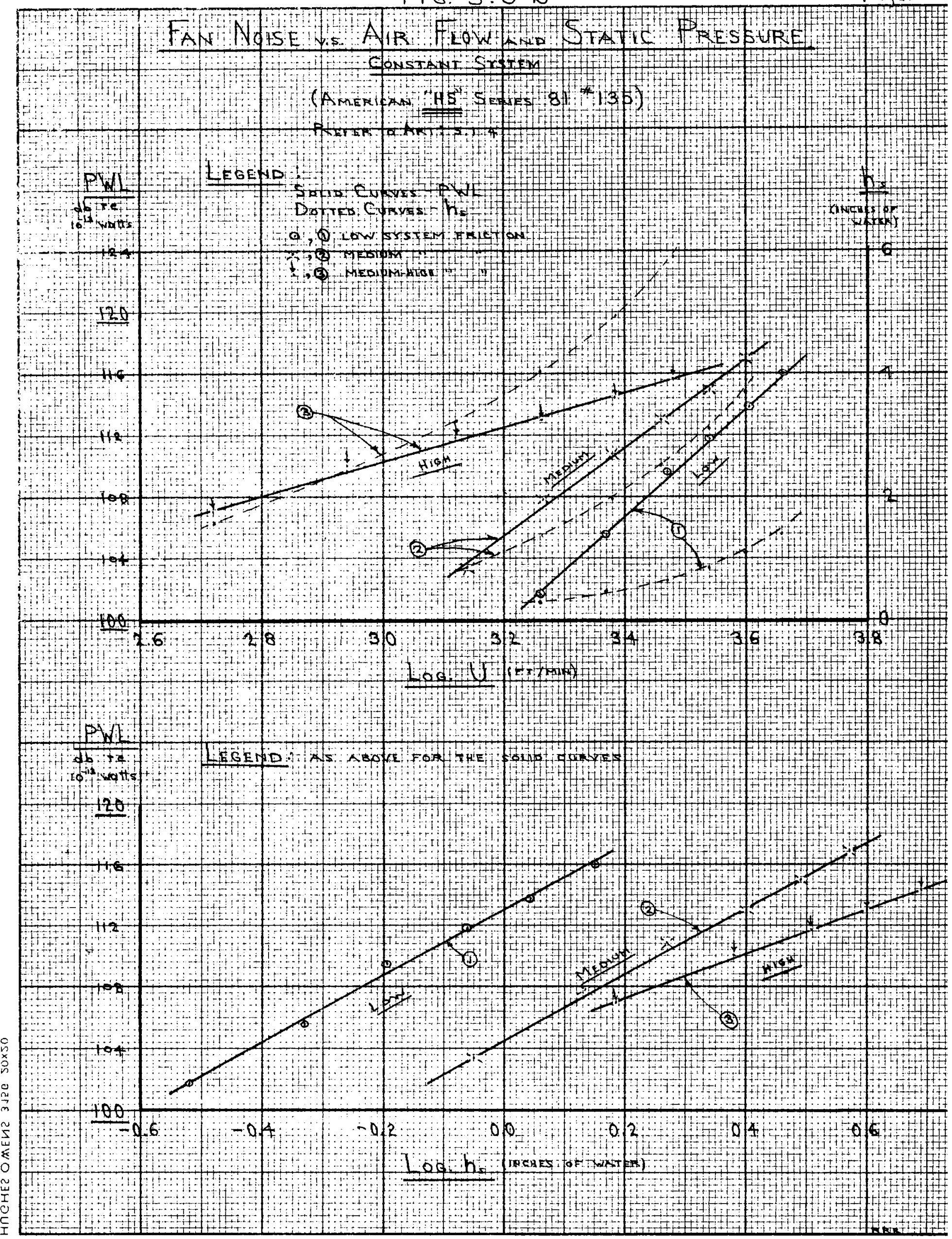


PWL vs. $\log U$ for high $F_{\text {syst }}$ as speed is increased to a sufficiently high value. What actually happens is that the unit capacity $Q / \sqrt{h}$, eventually departs from its linear variation as discussed in connection with Fig. 5.2. When Fsyst is high, hs is high and $Q$ relatively low for a given speed, hence in the range of the results, this condition is not reached up to maximum rated fan speed. An attempted explanation of these seemingIy inconsistent results is left until after the noise spectra have been examined. What we have is:

sirocco fan: $\quad\left[I \propto U^{\text {otos }}\right] F_{\text {syst; }}$ below $\eta_{s} \max$. $\begin{array}{ll} & {\left[I \propto U^{\text {stos }}\right] F_{\text {syst; }} \text { above } \eta_{s} \max .} \\ \text { HS fan: } \quad & {\left[I \propto U^{1 t_{0} 3}\right] F_{\text {syst; }} \text { below } \eta_{s} \max .} \\ & {\left[I \propto U^{3 t_{0} 4}\right] F_{\text {syst; }} \text { above } \eta_{s} \max .}\end{array}$

PWL vs. hs is plotted in the lower half of Fig. 5.9 for the same three $F_{\text {syst }}$ values as before. It is commonly held that for a given point of rating on the $\eta_{s}$-Ns curve, $\Delta I \propto \Delta h_{s}^{2.5}$. These curves of course represent widely varying points of rating, but nonetheless this relation is approximately valid over part of the fan operating conditions. Sirocco fan: $\left[I ₹ h_{s}^{2.5}\right] F_{\text {syst }} h_{s}<1.5^{\prime \prime} \mathrm{H}_{2} \mathrm{O}$ below $\eta_{s} \max$. $\left[I \propto h_{s}^{2.5}\right] F_{\text {syst }} h_{s}<3 n \mathrm{H}_{2} \mathrm{O}$ above $\eta_{s} \max$.

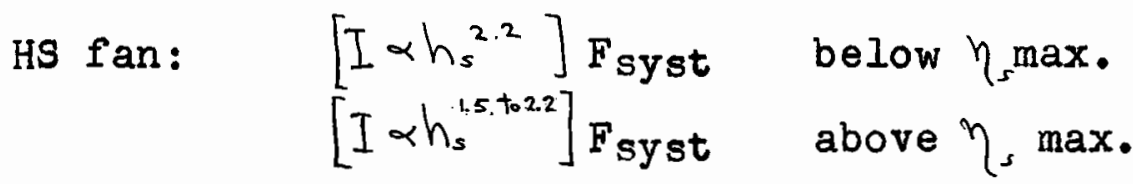

Above the hs limits for the sirocco fan, I varies with hs in the manner noted, bottom of Fig. 5.9a. 
Fig. 5.10 PWL vs. $\log$ Hp to fan, Fsyst constant.

This has been one of the main centers of study in the past, in order to relate fan PWL with an easily obtainable parameter, Hp. Beranek, Kamperman and Allen ${ }^{3}$ found that for fans operating near maximum efficiency (rated load) I $\alpha \mathrm{Hp}$, while for a given fan operating at variable load, I $\alpha \mathrm{Hp}^{2}$. G. Chipps found this latter to be true only near maximum efficiency and low back pressures. As hs increased $\mathrm{I} \propto \mathrm{Hp}^{x}$ where $x$ approaches 1 from 2. This finding is substantiated by the writer, Fig. $5.10 a, b$ upper half. The three curves correspond to the three values of $F_{\text {syst }}$ previously used. The dotted extension to the medium-high curve, Fig. 5.10a is the probable locus if speed could be increased above rated value.

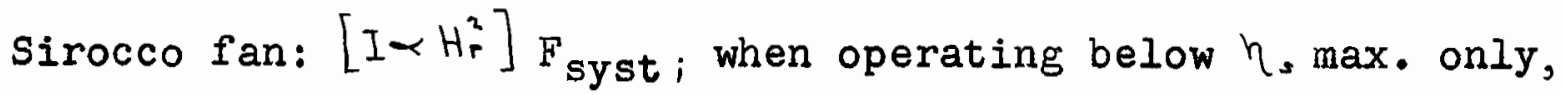
for the Hp well below rated. $\left[I \propto H_{*}^{0.5 t_{0} 2}\right]_{\text {syst }}$; when operating below $\eta_{s} \max$. only, for $\mathrm{Hp}$ approaching rated. $\left[I \propto H_{p}{ }^{2}\right]$ Fsyst near and above $\eta$, max. HS fan: $\left[I \propto H_{p}^{0.75 t_{0} 1.5}\right]$ Fsyst below $\eta_{s} \max$. $\left[I \propto H_{r}^{1.5}\right] F_{\text {syst }}$ above $\eta_{s} \max$.

The lower portion of Fig. 5.10a, b contains a trace of the (PWL) $=$ - Ns curve from Fig. 5.3a, b respectively. The points near this trace are a result of calculating (PWL)s, Ns data from $F_{\text {syst }}=$ constant values, compared to blade tip speed $\mathrm{V}=$ constant as in Fig. 5.3. Agreement is quite good.

It is obvious from the above results that relations between PWL and V, hs, $U$ and Hp are not consistent enough to be too useful. We shall examine the variations of $(\mathrm{PWI} / \mathrm{s}$ with 


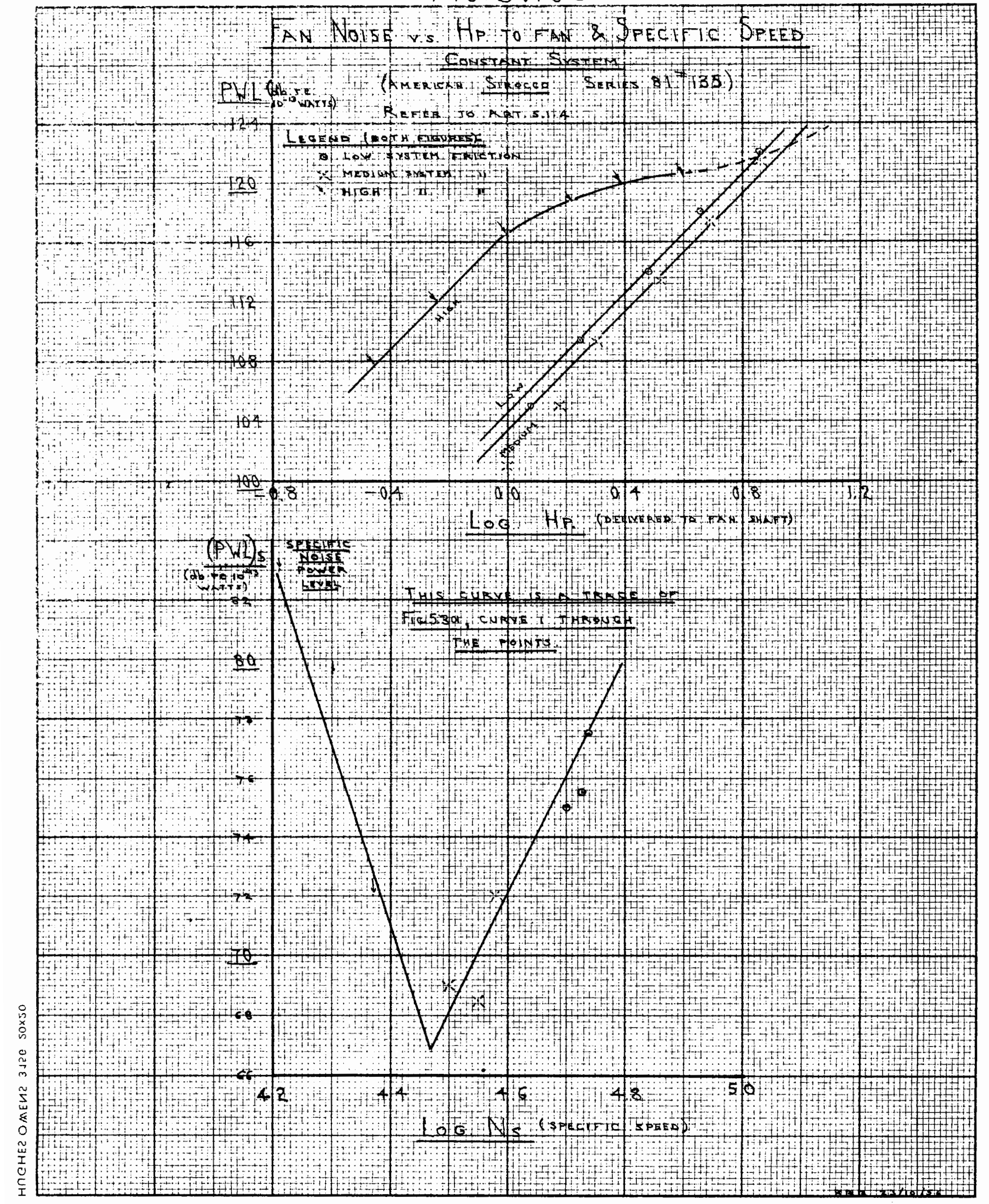




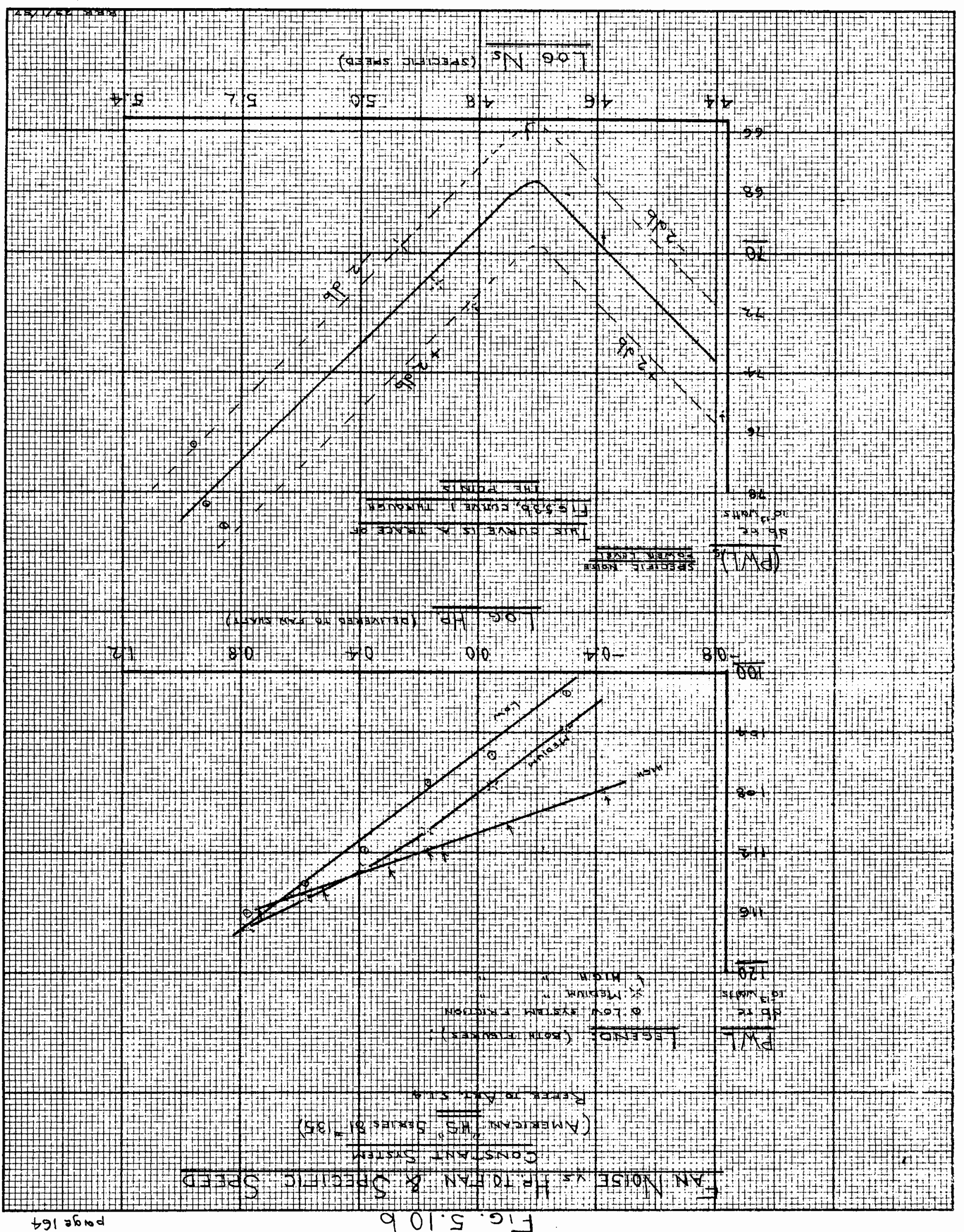


these same parameters. This time however, percent free flow (UF) is held constant at four values for the sirocco fan and three values for the HS fan. This is very approximately the same as holding $F_{\text {syst }}$ constant (back panel openings) as was done in Fig. 5.8 to 5.10. It should be noted that for the same range of back panel adjustment ( $\left.F_{\text {syst }}\right)$, the flow characteristics of the sirocco fan varied more than the HS fan. For this reason \% U; < $<40 \%$ cannot be given for the HS fan.

Fig. 5.11 (PWL) vs. $\log \mathrm{U}$ and $\log$ hs, \% Uf constant. Sirocco fan: $\left[(I)_{s}=\text { constant with } U\right]_{\% v_{F}}$ below $\eta_{s} \max$. $\left[(I)_{s} \propto U^{\text {oto }}\right] \% U_{F}$ above $\eta, \max$. HS fan: $\quad\left[(I)_{s} \propto U^{-2}\right] \% v_{F}$ above and below $\eta_{s} \max$. Sirocco fan: $\quad\left[(I)_{s}=\text { Constant with } h_{s}\right]_{\%_{F}}$ below $\eta_{s} \max$. HS fan: $\quad\left[(I)_{s} \propto h_{s}^{-1}\right] \% U_{F}$ above below $\eta_{s} \max$. Fig. 5.12 (PNL)s vs. $\log \mathrm{V}$ and $\log \mathrm{Hp}, \% \mathrm{U}$. constant. sirocco fan: $\left[(T)_{s}=\right.$ constant with $\left.V\right] \% U_{F}$ below $\eta_{s} \max$. HS fan: $\quad\left[(I)_{s} \propto V^{-2}\right] \% U_{F}$ above and below $\eta_{s} \max$. Sirocco fan: $\quad\left[(I)_{s}=\text { Constant with } H_{P}\right]_{\psi_{F}}$ below $\eta$, max. $\left[(I)_{s} \propto H_{p}^{0+01.5}\right] \%_{U_{F}}$ above $\eta_{s} \max$.
$\left[(I)_{s} \propto H_{p}^{-0.65}\right]_{U_{F}}$ above and below $\eta_{s} \max$.

Attention is also drawn back to Fig. $5.8 \mathrm{a}$, b upper half where (PWL) $)_{s}$ vs. $\log V, F_{\text {syst }}$ constant, was plotted as dotted curves. This was noted in the discussion on this figure. 


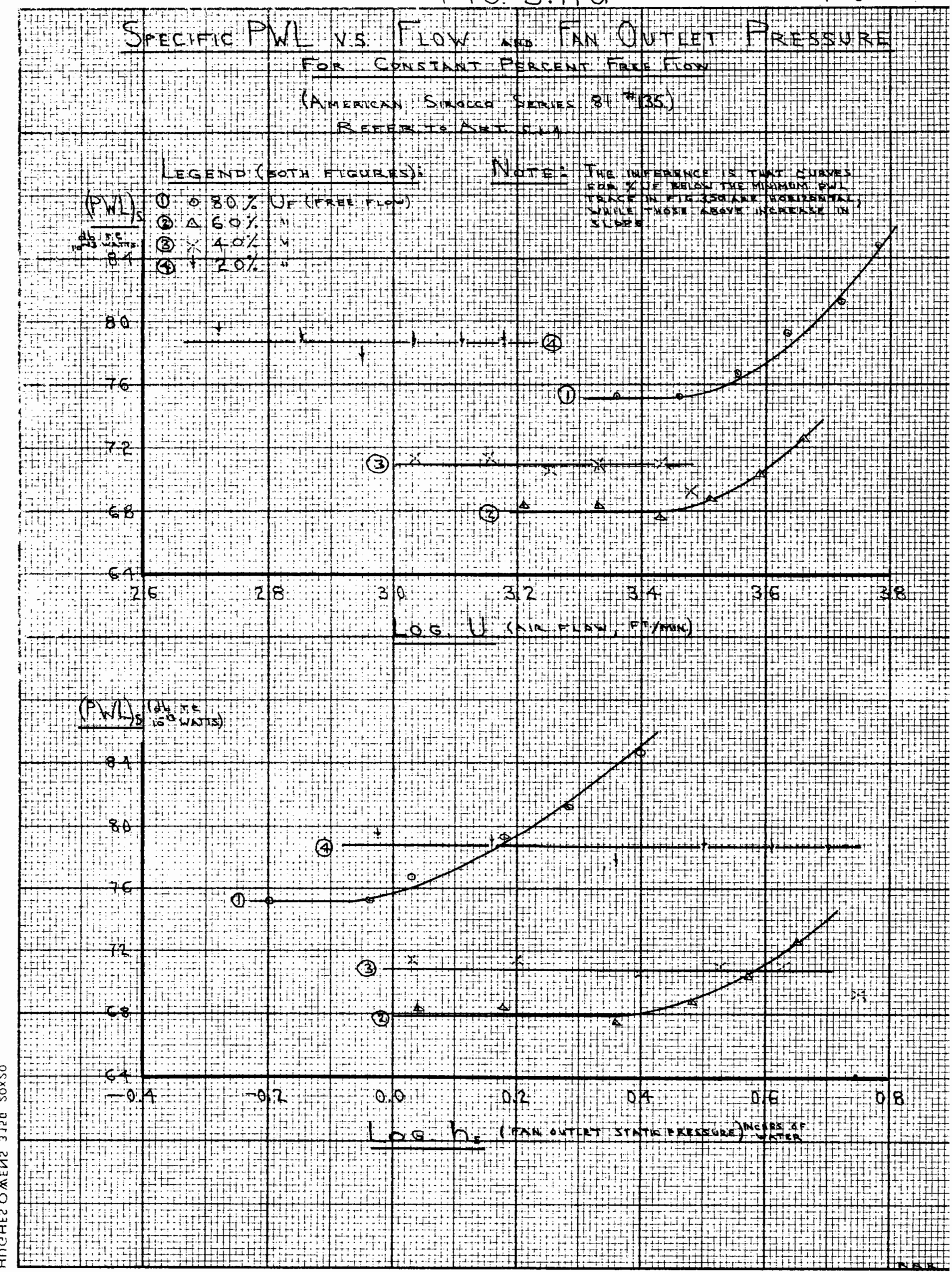




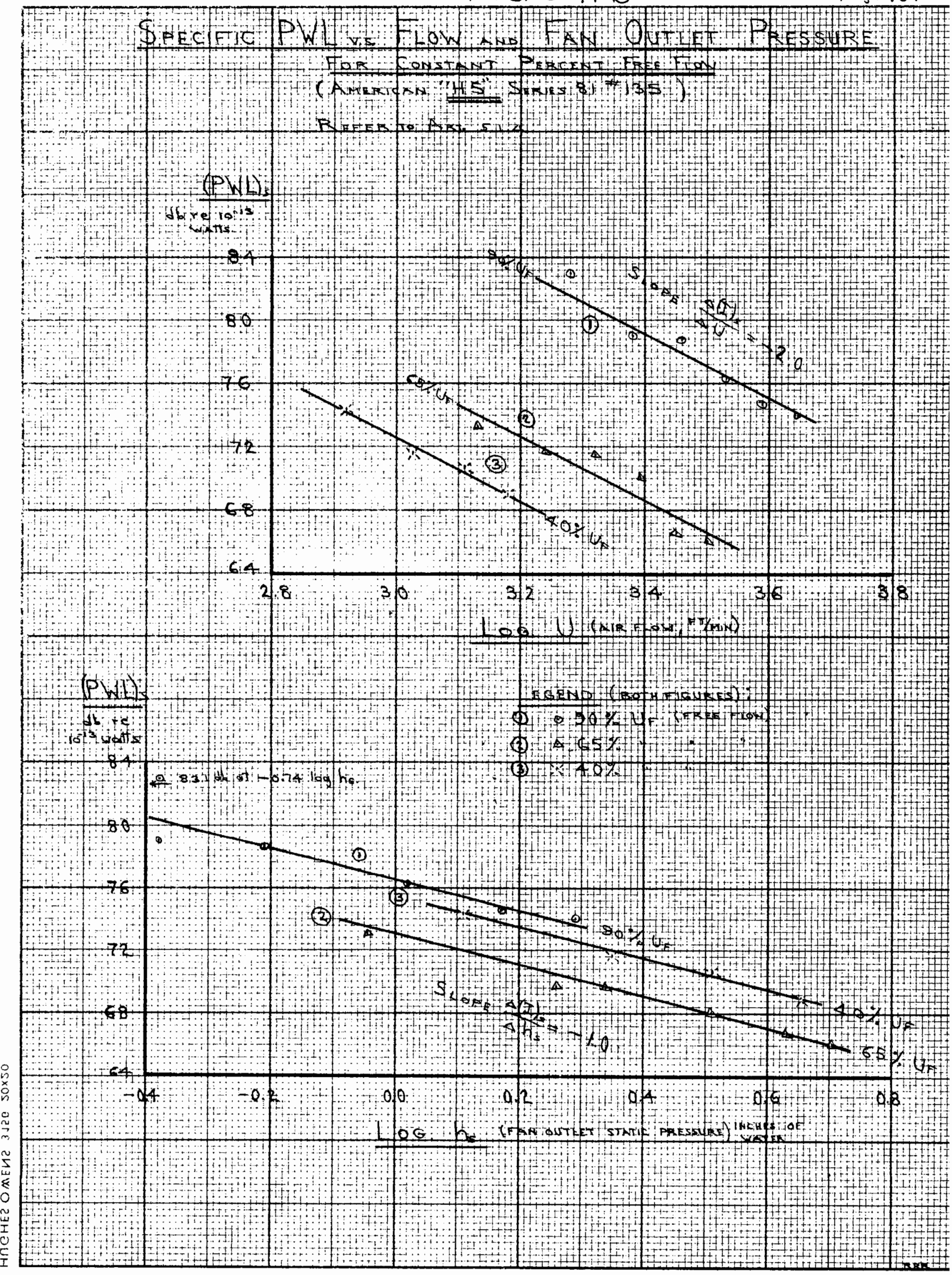




\section{Fig. $5.12 \mathrm{a}$}

page 168

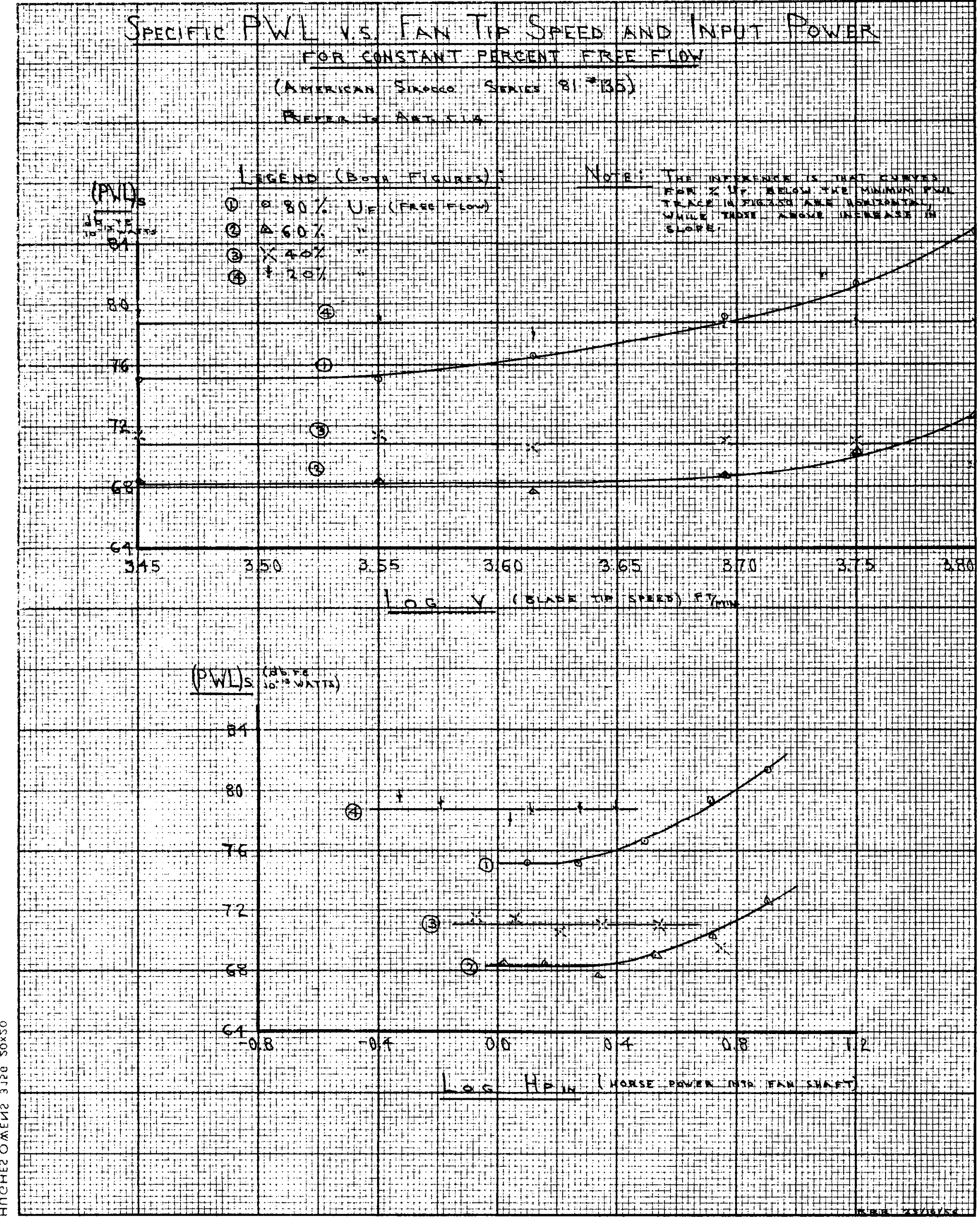


Fig. $5.12 b$

- are 169

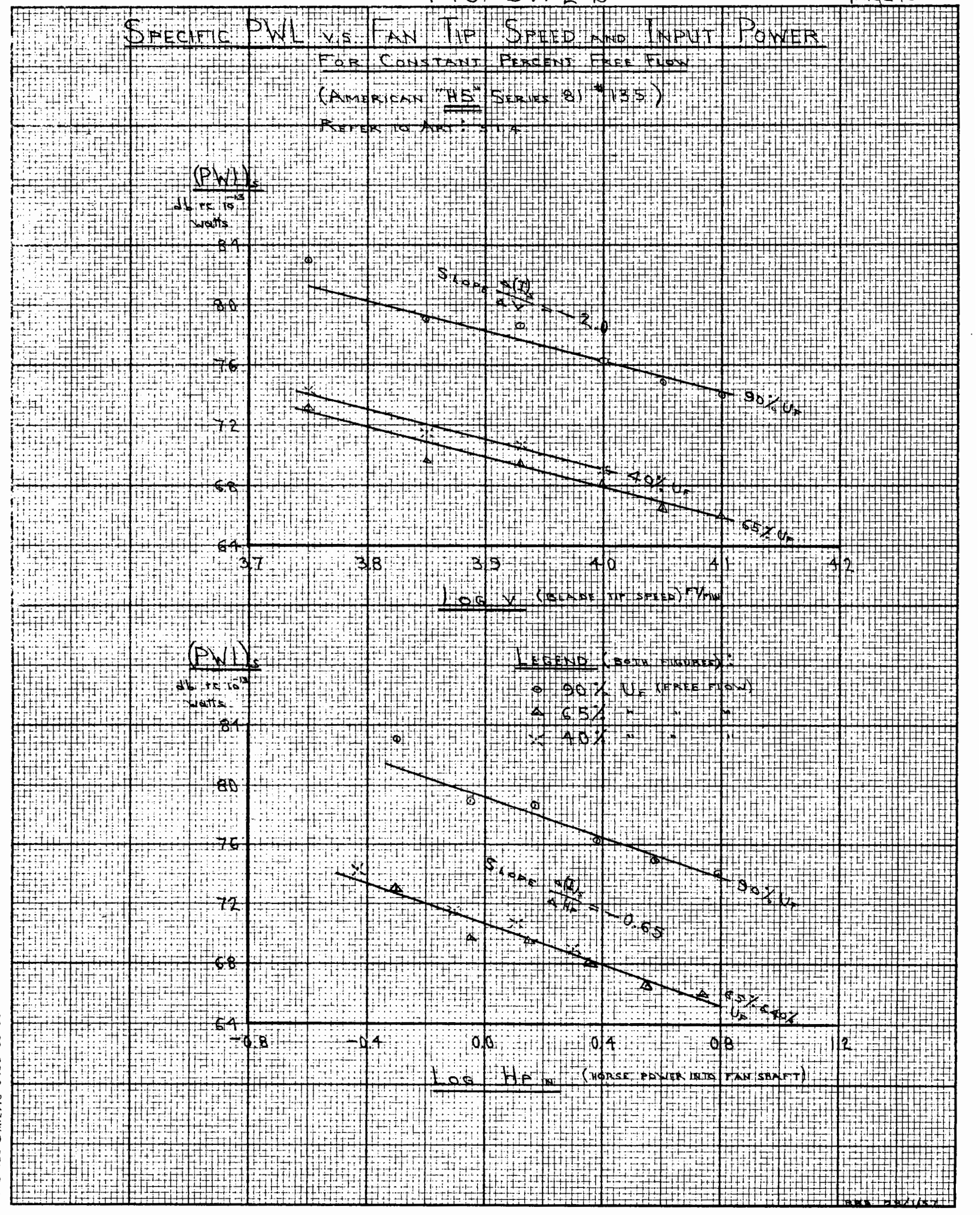


"laws" are those which possess a constant exponent. Once given a reliable (PWL) $-\operatorname{tog} N s$ curve and the fan flow characteristics, most of the laws become superfluous, as they can be computed from Eq.(5.1), (5.2). It is sometimes valuable however to estimate a change in sound intensity in a given system with some change in operating characteristics. Fan noise "laws" are then of value in saving calculation time.

These sections, Art. 5.1.1 to Art. 5.1 .4 discussed means of predicting overall fan noise power with little regard for the theory of why these variations are so. This will come during a discussion relating to the origin of noise in centrifugal fans, Art. 5.2.

\subsection{SPECTRA, AND CENTRIFUGAL FAN NOISE ORIGIN}

Having studied possible general laws relating fan noise PWL with fan - flow parameters, we turn to the important subject of the origin of noise in the fans, and spectra to be related with the PWL results. The spectra are of two types; $2 \%$ B.W. or octave bands as discussed in Art. 4. The reader is reminded of the contributing noise components predicted in Art. 2.2 .

5.2.1 FAN NOISE OUTLET SPECTRA

(at microphone station shown in Fig.1.3 p.18)

Figs. 5.13a, b show the fan outlet noise spectra on a $2 \%$ B.W. basis for various conditions of flow. The upper half of Fig. 5.14a, b shows the same thing on an band basis. 


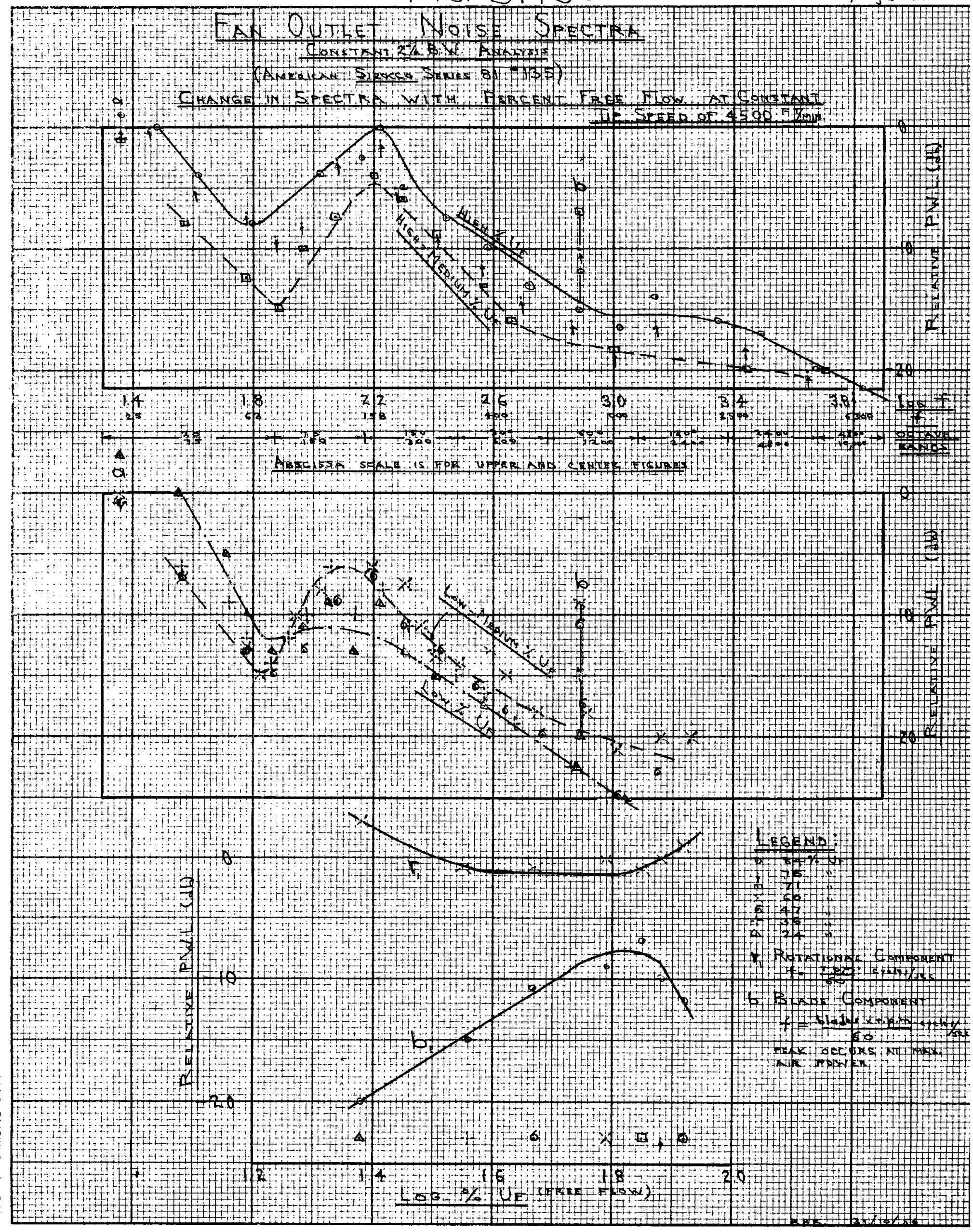




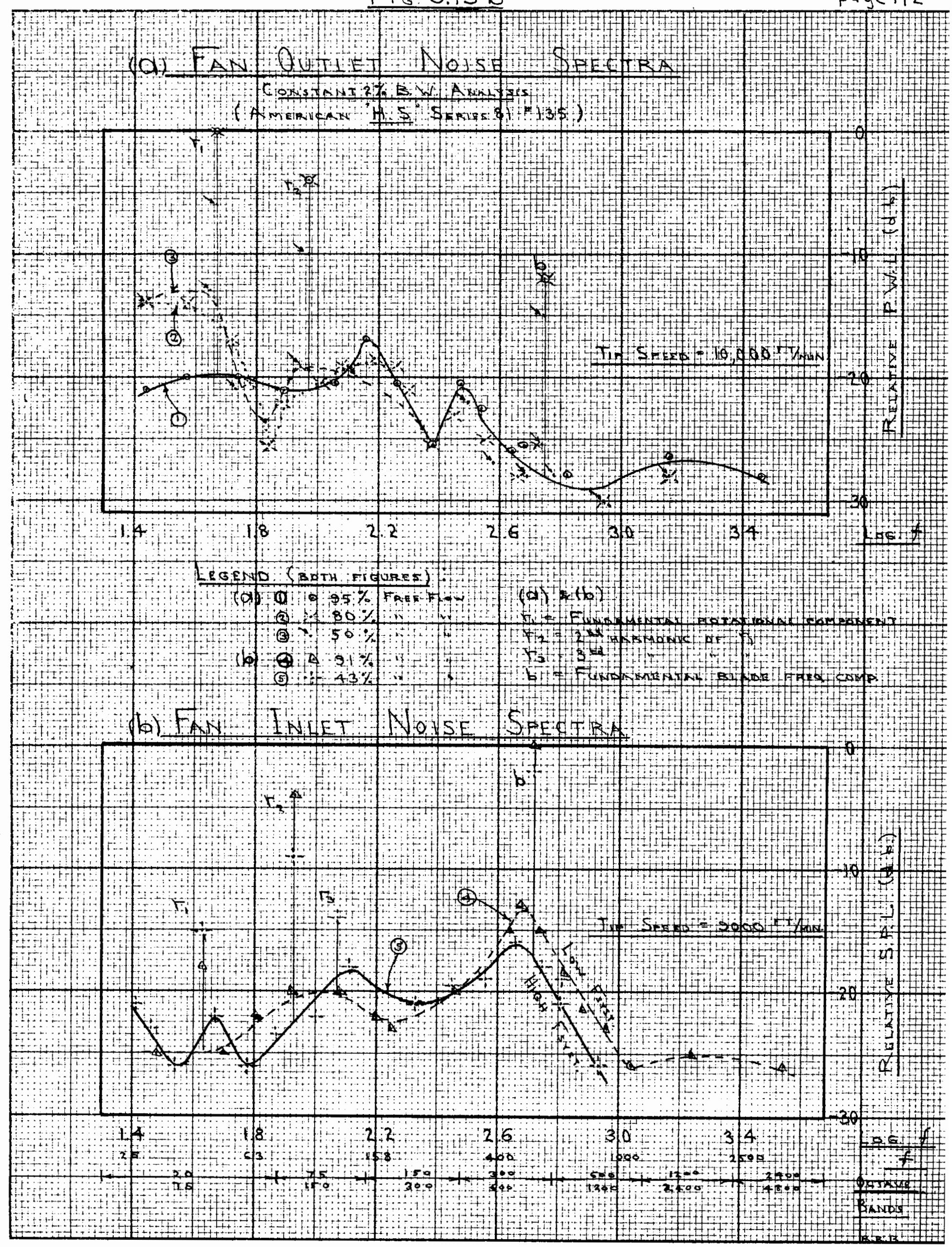




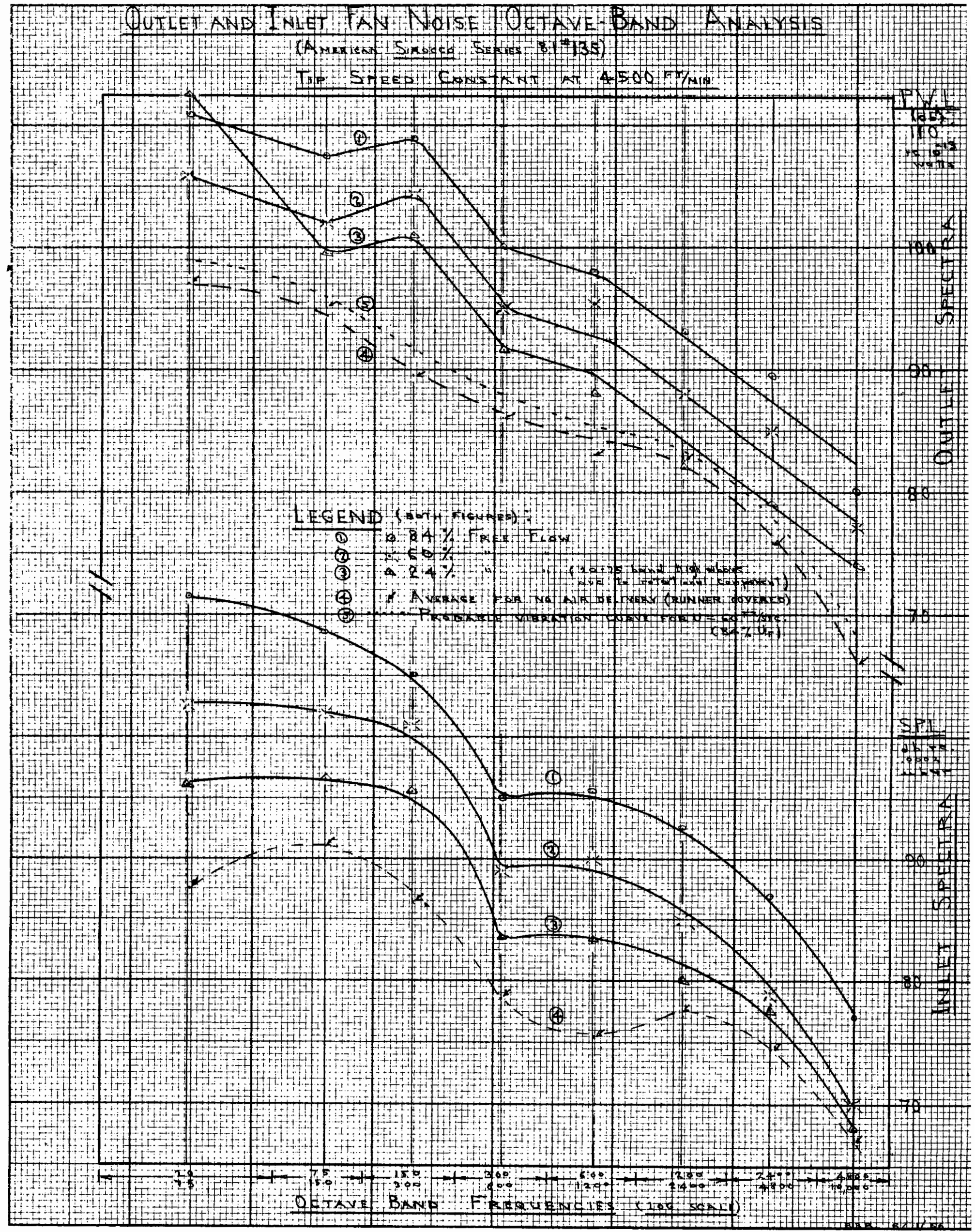




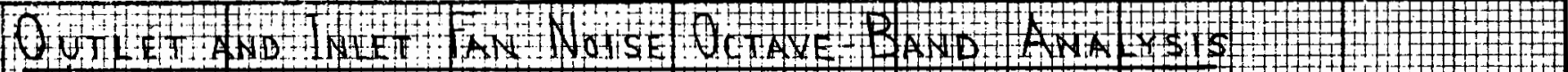

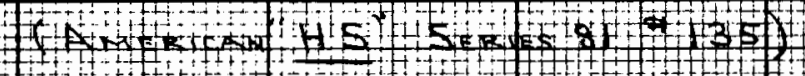

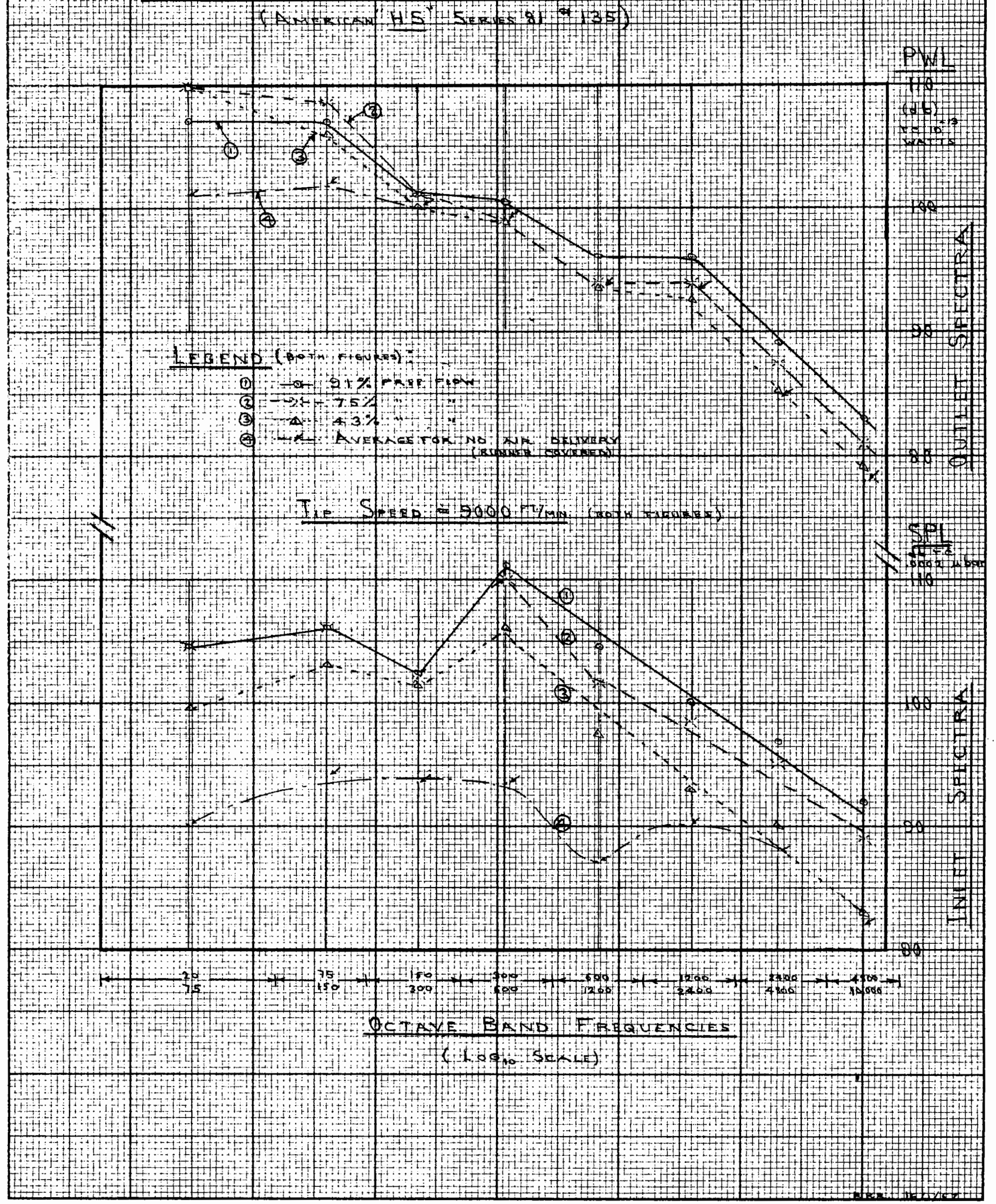


Note the following:

1. The Sirocco and HS fan spectra on a $2 \%$ B.W. basis are quite different but on an octave band basis are similar.

2. The higher the back pressure,hs, (the lower the $\%$ J higher is the first band noise (Fig. 5.14a, b).

3. There is a component or hump near $\log f-2.2$ that increases as \% U. increases (as the air flow velocity $U$ increases).

4. Both the rotational line component $(r$,$) and blade component$ $(b$,$) are prominent. The second harmonic of the rotational$ component $\left(r_{2}\right)$ is prominent in the HS fan noise output. These "line" components were discussed in Art. 2.2.4. They are:

$r_{n}=$ the $n^{\text {th }}$ harmonic of the rotational frequency line component $=\left(\frac{\text { fan r.p.m. }}{6 \delta}\right)$ n c.p.s.

$b_{n}=$ the $n^{\text {th }}$ harmonic of the blade frequency line component $=\left(\frac{\text { fan r.p.m. } x \text { no.of blades }}{60}\right)$ c.p.s.

Note that these components are much more prominent in the HS fan spectrum than the Sirocco; a consequence partly of the higher speed and fewer blades. The variation of these components with back pressure at constant speed are shown for the Sirocco fan at the bottom of Fig. 5.13a. The rotational component $r_{1}$ passes through a broad minimum in the vicinity of maximum efficiency $(\eta, \max )$ while the blade component $b$, passes through a maximum in the region of maximum air power (based on hs), somewhat above the $\eta$, max point in terms of specific speed (Ns). Logical variations for the HS $r_{1}, r_{2}$ and $b$ line components were not apparent as hs was varied. These components re- 
mained relatively unchanged.

5. The continuous portion of the spectrum is the greatest contributor to Sirocco fan noise, while it is not the greatest part of the HS fan noise.

6. The continuous Sirocco spectrum declines at a greater rate with frequency than does the HS fan spectrum, although the bandwise difference Fig. 5.14a, b is not as marked. This is likely a consequence of the higher (double) speed of the HS fan. Note too that the higher the back pressure, the faster the spectrum declines with frequency. This is in line with other workers 4,7 in the field. These features are simply listed at this time and will later be referred to when discussing the origin of the noise.

In Fig. 5.14a, b upper half there is a curve designated as being for no air delivery. This is not a spectrum for fan cut-off but is that obtained when the fan runner was covered over its inner periphery so that it could still rotate and cause some air turbulence, but would not deliver air. This alteration of the noise source had a much more marked effect on the spectrum for the Sirocco fan than it did for the HS fan. It will be seen later that some of this noise is due to remaining air turbulence at the blades and not due to mechanical vibration of the fan. Efforts to enclose the runners with smooth material so that they would rotate with negligible air turbulence failed because of the centrifugal forces involved. Had this succeeded, the contribution of noise due to vibration alone could be measured. As it was, it had to be estimated from vi- 
bration measurements, as discussed later.

\subsubsection{FAN RUNNER PWL AND SPECTRA}

The main purpose of the many noise spectrum results is to separate the noise origins. It was predicted in Art. 2.2 that the major contribution to fan noise would be due to boundary turbulence at the blades, especially since most centrifugal fans are designed to operate at blade tip speeds of 4000 to 13,000 ft/min. The next step in the separation of noise components was to remove the runners and rotate them in an anechoic chamber, as described in Arts. 3, 4 .

The overall noise power (PWL), generated by the runners as a function of speed is illustrated in the upper half of Fig. 5.15a, the lower half of Fig. 5.15b. (See Art. 4.1 for the method of obtaining this). The solid curve represents the runner PWL vs. $V$ while the dotted curves form the envelope of overall fan outlet noise PWL over a wide range of back pressures. The runner PWL lies about $5 \mathrm{db}$ below the fan PWL for the Sirocco fan, but almost equals the overall PWL for the HS fan. The rate at which the runner PWL increases with speed is quite the same as overall PWL vs. speed, Fsyst constant, Fig. 5.8. This is further evidence that turbulence at the runner blades is the major source of noise in a fan. It is not so at very low fan speeds because of the "constant" system noise.

It is interesting to note the effect on the runner noise when the runner is covered on the inner periphery. At the higher runner speeds, overall PWL decreased about 9 to $10 \mathrm{db}$ 


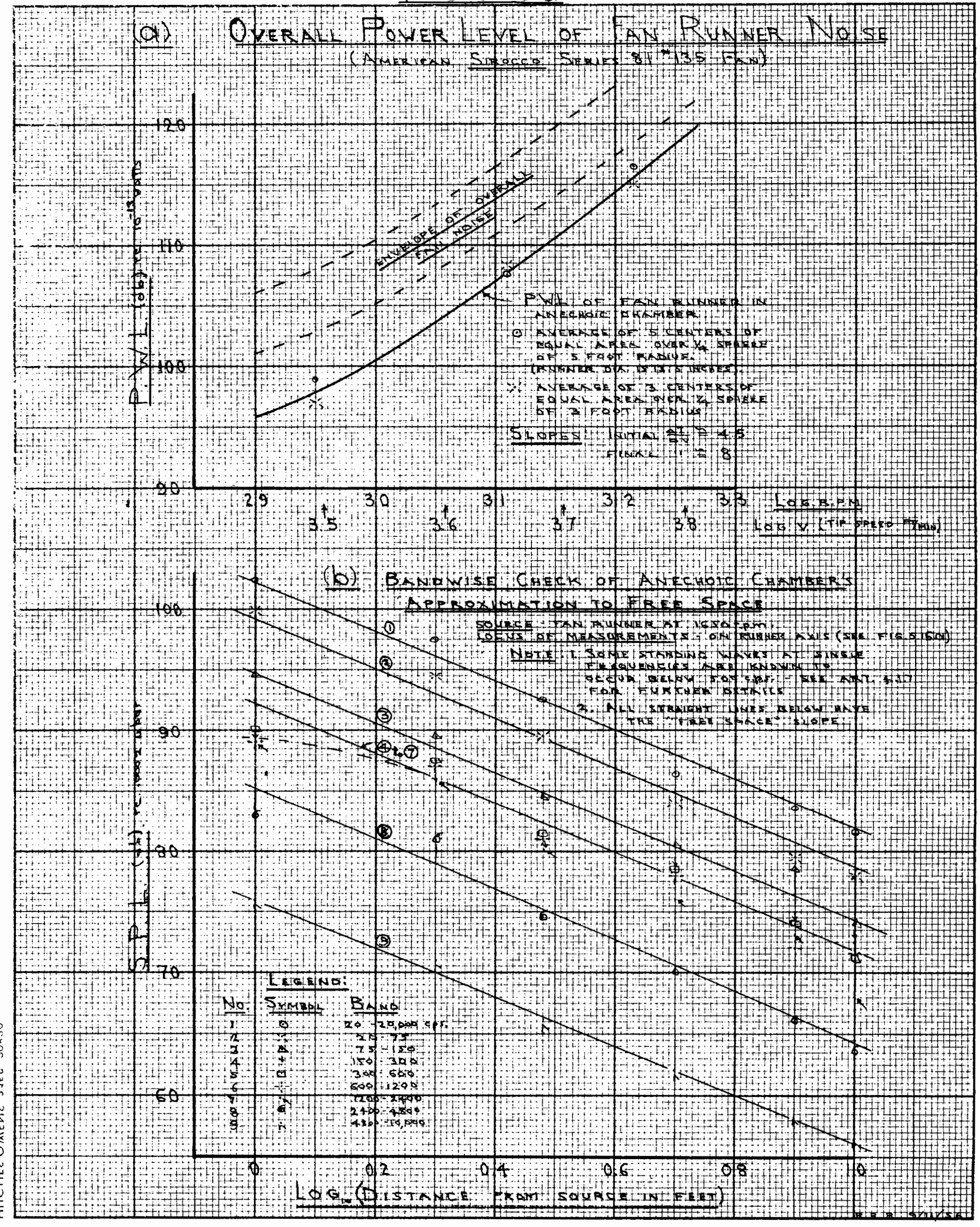




\section{Fig 5.15 b}
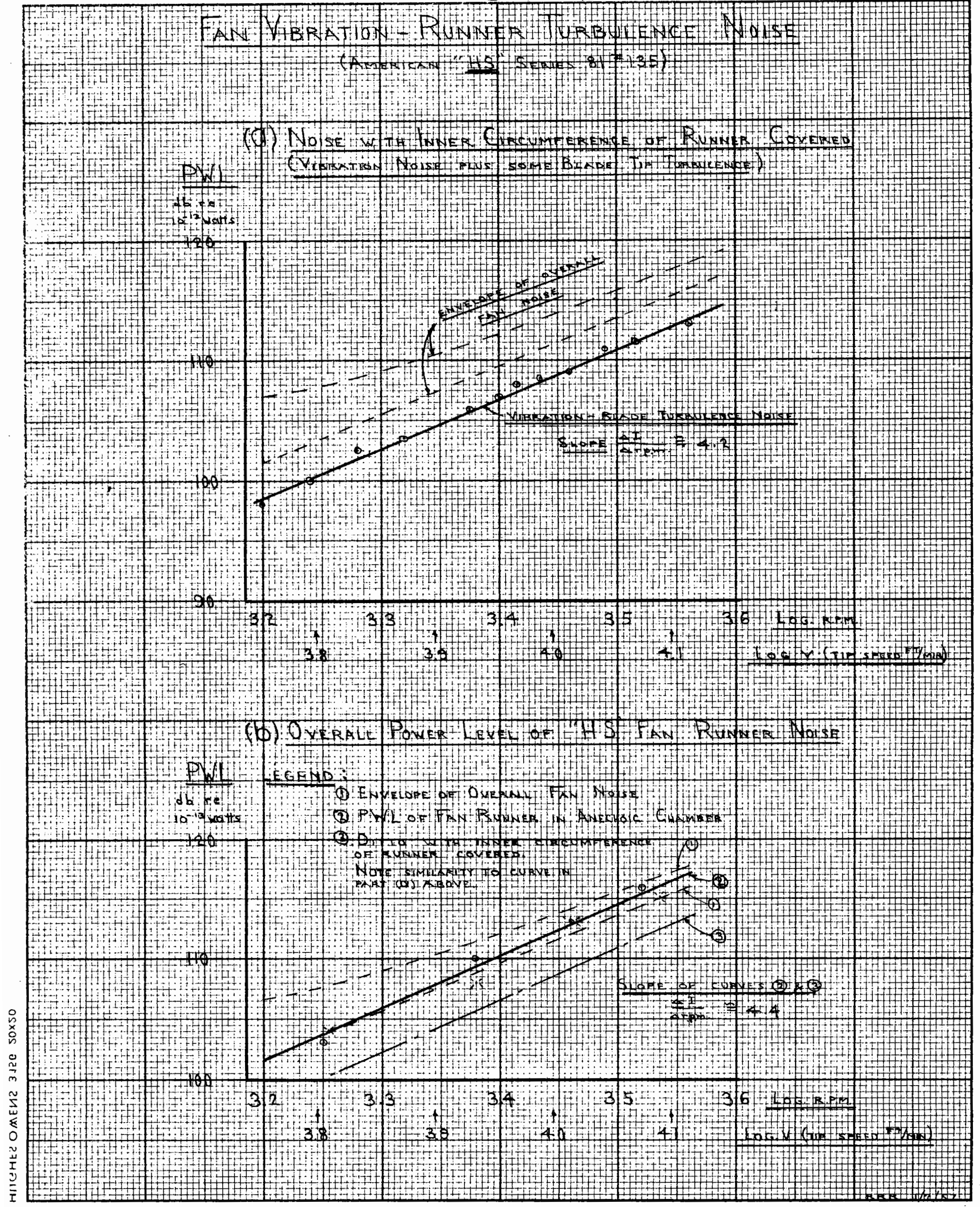
for the Sirocco runner; about 4 to $5 \mathrm{db}$ for the HS runner. The same effect was recorded when measuring fan outlet noise with the inner periphery of the runner covered. The results for this are shown at the top of Fig. 5.30 p.229 for the Sirocco fan and the top of Fig. 5.15b for the HS fan. PWL vs. speed is compared between the covered runner (solid curve; some blade turbulence plus vibration, no air delivery) and the envelope of fan noise results under varying operating conditions (dashed curves). Under this condition it is still mainly turbulence at the outer blade tip that is responsible for the measured noise. The difference between the inner radius covered, and uncovered case, is greater for the Sirocco fan than the HS fan. This lies in the smaller blade depth, blade shape and closely spaced blades of the Sirocco runner as compared to the HS runner. Other differences such as the different rate of PWL increase with speed are left to Arts. 5.3.1, 5.3.4.

Let us now study the runner spectra measured in the anechoic chamber. Fig. 5.16a, b each contains very useful information. Before examining them, three points should be clarified. First is that the stations used for the measurements are indicated by the sketch in the upper right corner of Fig. 5.16a. They are referred to in the legend and are the same for Fig. $5.16 \mathrm{a}$ and b. Next, it was mentioned in Art. 3 that the quality of the anechoic room lacked much, and that a bandwise check on the approximation to free space is given in Fig.5.15a bottom. The spectra drawn are therefore not precise results, but are reasonably good average results. Thirdly, curves 5 and 6 Fig. 5.16a and curve 6 Fig. 5.16b are the ambient noise spec- 


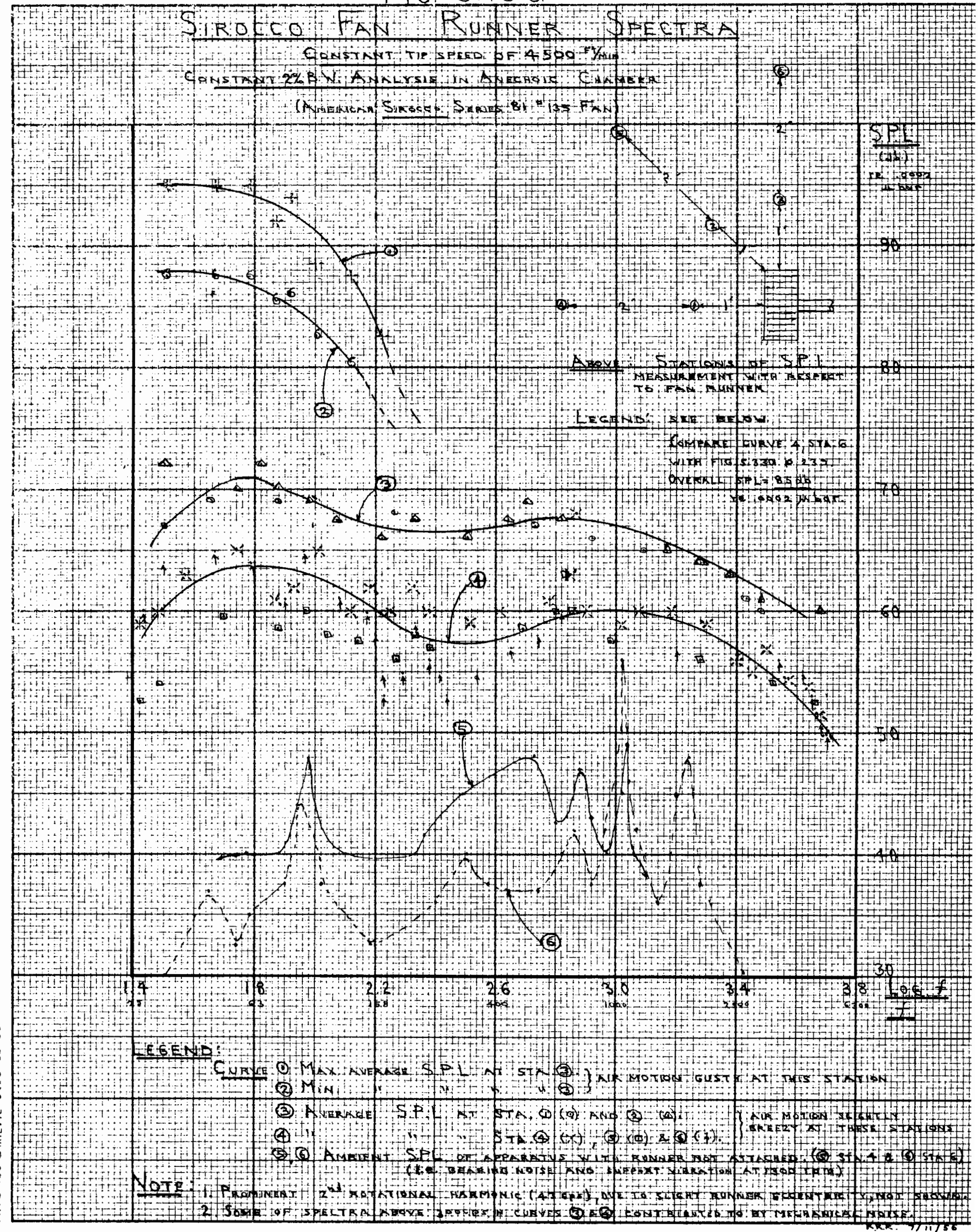




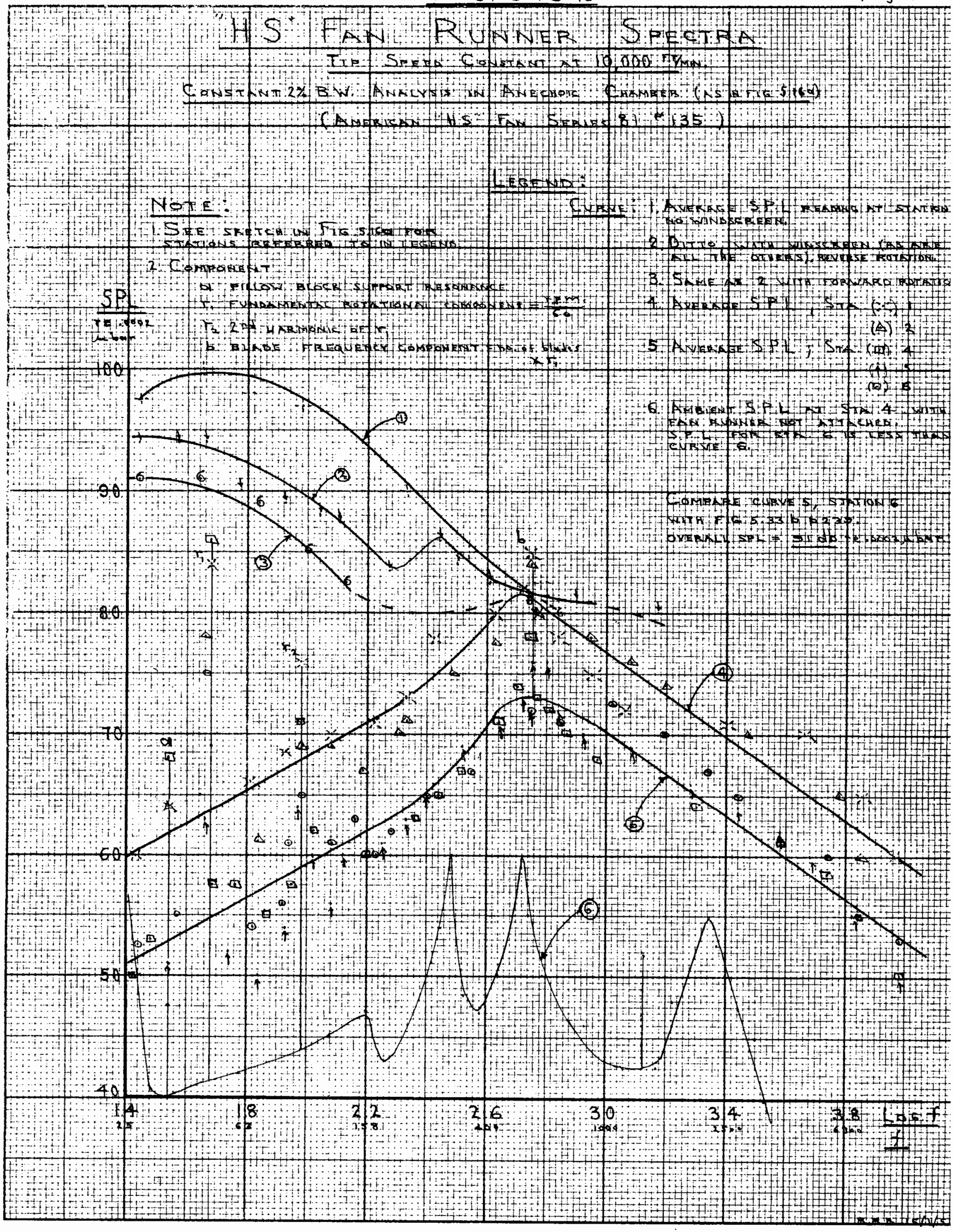


tra for the apparatus running minus the runner. A short steel shaft fitted to ball bearing pillow blocks provided the driving linkage between the interior and exterior of the anechoic room. A steel support was used outside, a wooden one inside. Due to the inherently noisy operation of ball bearings, and the lack of a rigid foundation (such as concrete) for the base in the anechoic chamber, the ambient noise is relatively high, but for the most part does not contribute significantly to the runner spectra. Exceptions to this are near 1000 c.p.s. for the Sirocco runner, and line components $a, r_{1}$ and $r_{2}$ for the HS runner.

Curves 3 and 4, Fig. 5.16a, and 4 and 5 Fig. 5.16b differ only in that the lower one is the SPL some 3 feet from the runner, while the upper one is the SPL I foot away. In free space this means a difference of about $9 \mathrm{db}$. The difference actually obtained varies from $7 \mathrm{db}$ to $9 \mathrm{db}$ due to the imperfect anechoic chamber and the averaging of the results from three stations. These curves are the relative spectra of the runners alone. They are similar in this respect to spectra obtained by past workers for rotating cylinders and the like. The prominent features are:

1. The spectra are continuous and widebanded. This was predicted in the theory for spectra of rotating elements.

2. The Sirocco runner spectrum is relatively flat over a wide frequency range. There are no line components. There is a broad maximum ranging from about 40 to 120 c.p.s.

The HS runner on the other hand has a peaked spectrum, 
the fundamental blade frequency $b$, appearing as a line component at the spectrum maximum. The SPL falls off quite rapidly on either side of the maximum, although it remains continuous. Rotational components $r_{1}$ and $r_{2}$ are also present, but this is an abnormal situation due to slight runner unbalance and the nature of the support used.

Review for a moment the noise from a subsonic propellor. The noise is similar to that generated by a pulsing flow and consists primarily of a few frequencies of constant amplitude harmonically related to the blade passage frequency. At subsonic tip speeds, the fundamental predominates. Or recall the noise from a bluff obstacle in an airstream. Looking back to Fig. 2.4(f) p.70 we find the peaked but continuous spectrum of noise observed by Gerrard 33 for a circular cylinder in an air stream.

How shall we consider the Sirocco and HS runners as noise generating rotating systems? If they consist of a few relatively deep blades rotating at low subsonic tip speeds, they are somewhat analogous to a combination of a pulsing device and a bluff obstacle in an airstrean. We should therefore expect a peaked spectrum with perhaps a line component at the blade passage frequency plus possible harmonics. Next consider the rotating system consisting of a large number of shallow closely spaced blades moving at fairly high subsonic tip speeds. Let the system approach a cylinder. In the limit the plate would simply become a smooth cylinder rotating about its longitudinal axis. The noise output from this system would be low 
except at high surface speeds at which point we would have noise from boundary layer turbulence. In Art. 2.2 it was pointed out that the spectrum due to this (Mach.<l) is very wideband, has no prominent components, but passes through a broad maximum over a frequency range depending on the surface speed. This maximum shifts upward in frequency as speed increases. For a rough surfaced circular cylinder, boundary turbulence would increase vastly and the noise output therefrom would be significant at a much lower speed. The spectrum however should still be characterized by its width, maximum and lack of line components.

The Sirocco runner consists of closely spaced shallow forward curved blades rotating at tip speeds on the order of Mach. $=0.1$. The HS runner consists of deeper back inclined plates, fewer in number hence farther apart, but operating at tip speeds of about Mach $=0.2$. The sirocco runner would be expected to have a broader, flatter noise spectrum than the HS runner, in light of the above paragraph. A third general series of centrifugal fans is the radial blade type. These are of two general classes, a wide shallow bladed one with specific speeds intermediate between the Sirocco and HS runner; and narrow deep bladed pressure blowers of low specific speed. These radial types usually have only a few blades and therefore resemble paddle wheels. The blades are generally deep and the blade tip velocities lower than the HS or Sirocco fans. We can then predict their spectrum from above considerations as being sharply peaked at the fundamental blade passage frequency, plus some prominent blade passage harmonics. 
been said regarding the sirocco and HS fan runner spectra. It should be borne in mind that converting these to an equivalent I c.p.s. bandwidth base would tilt the spectra clockwise about the $100 \mathrm{c.p.s}$. frequency. They were plotted in the $2 \% \mathrm{~B} . \mathrm{W}$. form for reasons given in Art. 4.2 .

\section{2 .3 AIR TURBULENCE SPECTRA}

(Anechoic chamber and termination study).

Driving a fan runner alone in space at rated speeds sets up tremendous turbulence about the runner. For the tests in the anechoic charnber, on the order of $1 \mathrm{Hp}$ was delivered to the Sirocco and HS runners at maximum rated speed. This energy was spent as heat generated by air turbulence. In the length of time it took to record one $2 \% \mathrm{~B} . \mathrm{W}$. spectrum for example (20 to 30 minutes), the temperature in the closed $1,4 \times 10^{3}$ cu.ft. anechoic chamber would rise some $20^{\circ} \mathrm{F}$ from the original ambient of $68^{\circ}$ to $73^{\circ} \mathrm{F}$. This turbulence existed in strength only within about 1 to 2 feet of the runner blade tips, hence only station 3, Fig. 5.16a would be in this turbulence. The microphone was suspended firmly at this station for $90^{\circ}$ incidence, and the noise recorded for both runners. The spectral results are given by curves 1 and 2, Fig. 5.16a and curves 1,2 and 3 , Fig. 5.16b. The increase in low frequency power is tremendous, as one can appreciate by observing the ordinate magnitude between the results at station 3 and those at station 1 on the runner axis. After examining the spectra for the various stations $I$ to 6 , it must be admitted that most of this additional signal is not so much due to noise perhaps as due to inefficiency of 
the windscreen in preventing the air pulsations of turbulence reaching the microphone. If this were not so, the low frequency power would be increased everywhere in the anechoic room.

In Fig. 5.16b, curve 3 is the recorded output at station 3 for the HS runner rotating in normal direction. Curve 2 is for the runner rotating at the same speed in the opposite direction, so that the runner now has blades that are forward inclined instead of backward inclined. The air turbulence is greater, as would be expected, since the forward swept blade is a very blunt obstacle compared to the element of "streamlining" associated with the backward swept blade. Curve $I$ is for no windscreen on the microphone, the HS runner rotating in the same direction and at the same speed as for Curve 3. It shows a 7 to $13 \mathrm{db}$ attenuation for the windscreen at these low frequencies.

The results appear rather discouraging for measuring fan noise in an airstream with this design of windscreen (which is among the best, as noted in Art. 3). The turbulence resulting from rotating the runners was of a gusty nature however, unlike that which would be encountered in a wind tunnel. There is evidence to show that the windscreen was performing satisfactorily in the acoustic waveguide. Turning back to Fig.5.13a and $b$, the highest low frequency components (below 50 c.p.s.) actually occur for the lower flow, higher back pressure condition, not the highest flow as one may first expect.

There is further experimental evidence of low frequency noise output from turbulence in a ventilating airstream (as 
opposed to a "gusty" turbulence when spinning the runners alone).

Means for determining this was afforded by the conflicting aerodynamic - acoustic requirements for the transition between the acoustic waveguide and its termination (i.e. the exponential horn). The requirements to match the flow in a pipe of one diameter to that of another, with minimum loss, is that the transition have as small a taper as possible $\left(7^{\circ}\right.$ max. recommended by N.A.F.M.). Acoustic matching in a minimum of space requires an exponential, catenoidal or hyperbolic profile. Aerodynamically, these shapes result in pressure loss, additional turbulence and uneven air flow distribution (over and above that which may already be present) beyond the horn. What actually occurs is illustrated by the air flow contours, Fig. 5.17a, b, right side, for various conditions of system friction (Fsyst, back panel adjustment). The view is looking into the horn mouth towards the fan. The arrangement of apparatus is shown in Fig. $3.5 \mathrm{p} .101$. It is interesting to compare the different effects equivalent Fsyst has on the flow beyond the horn as produced by the different runner types. The higher the $F_{\text {syst }}$ (or back pressure) against the Sirocco fan, the more central the apparent flow center in the termination, whereas it is low $F_{\text {syst }}$ which produces this result in the HS runner case. Centrifugal fans exhausting into a duct of area equal to their outlet area cause a vertically non-uniform flow, the largest velocity component being above the duct axis. The flow is quite symmetrical in a horizontal plane. This would follow from the outward air velocity component at the blade, tending to increase the flow near the duct roof. This condition is 
Comparison of Sound level and Air Flow Contours BEYOND MOUTh OF HORN (Sirocco RunNer)

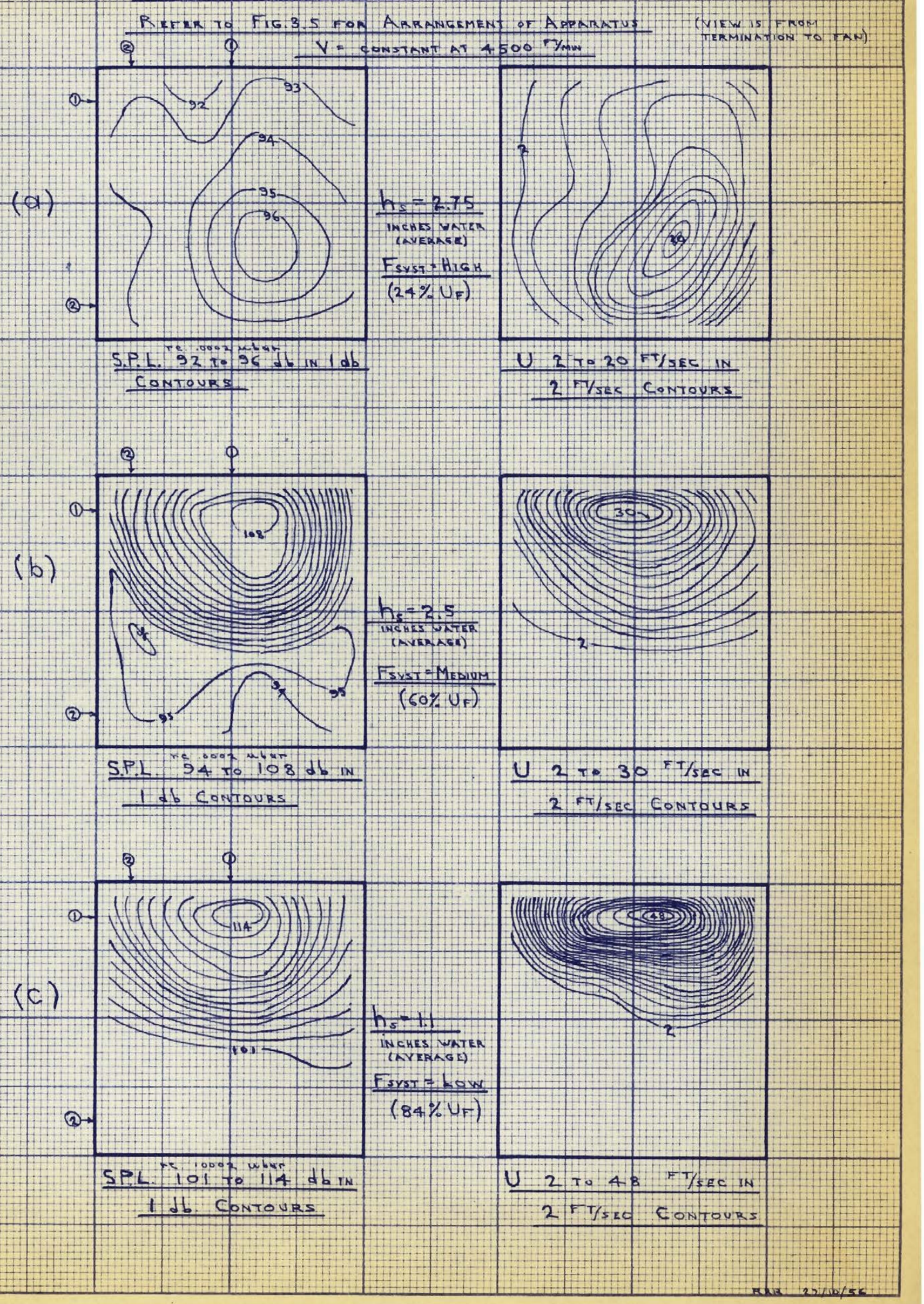




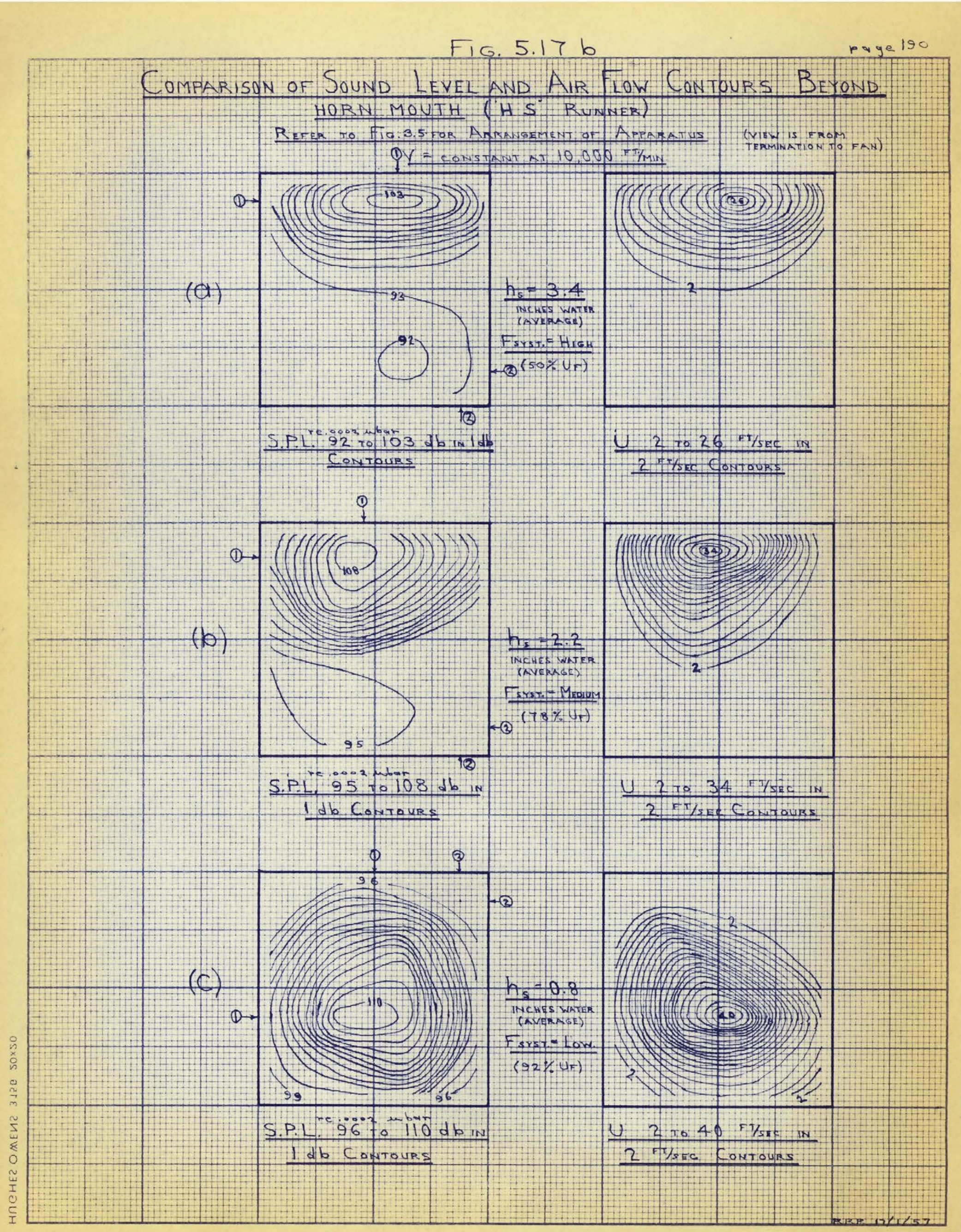


modified to varying degrees by the back pressure against which the fan must operate. This back reaction affects the exhaust flow characteristics of differently designed nunners to varying extents, depending on the runner design and condition of operation.

The stream characteristics beyond the horn mouth afford an opportunity of:

(1) Assessing the efficiency of the windscreen.

(2) Verifying whether the air flow itself actually causes a measurable noise output compared to the fan noise. This can be done where the stream is highly concentrated, leaving the rest of the termination cross section without appreciable air motion. If a microphone is placed in this quiescent section, it will record noise from the fan, plus turbulence noise due to the airstream, without being affected by the turbulence itself, since it is not in moving air. The windscreen is still used as a precaution against any low flow which could not be estimated with the Pitot tube and manometer.

Compare now the SPI contours over the same section, with the air flow contours. In Fig. 5.17a, b the SPI contours are on the left hand side. Note:

(1) The striking resemblance in the contour maps. Looking at the SPL and flow maxima, the SPI increases with the flow density, but not proportionately.

(2) Most important, note that the SPL in the quiescent region increases with the flow density. It does not increase significantly with maximum flow itself, but with 
the density or gradient of the flow, as witnessed from the bottom diagrams, Fig. 5.1la, b (F syst low). Thus the SPL is $101 \mathrm{db}$ in the quiescent region of the $48 \mathrm{ft} / \mathrm{sec}$. max. flow of the sirocco fan, but only $96 \mathrm{db}$ in the quiescent area of the $40 \mathrm{ft} / \mathrm{sec}$. max. flow of the HS fan. The difference is the relatively steep gradient from the max. to min. flow for the sirocco case as compared to the moderate gradient in the HS case. This follows from Eq. (2.16) where we found the momentum flux tensor, which is the principal noise generating component in turbulent flow, was proportional to the gradient of the time mean momentum flux crossing a surface whose normal is perpendicular to the direction of the component of momentum flux being considered.

What we would like to do at this point is to evaluate the additional noise PWL due to this turbulence. It is not possible,unfortunately, because of equipment limitations such as standing waves below horn cut-off hence hoarding of noise power within the duct. Air speeds within the duct are high, but do not vary appreciably in the cross section until the duct wall is approached. The air velocity reduces to zero at the wall and the resulting large velocity gradient would give rise to some boundary turbulence noise. The result is that the PWL measured in the duct is higher than that which would be obtained by adding $11 \mathrm{db}$ to the average SPL recorded near the horn mouth. The measured PWL in the duct is as follows for the speed and flow conditions specified in Fig. 5.17a, b, compared with the approximate estimate from the contour maps of SPL across the horn mouth: 


\section{TABIE 5.1}

FAN

Siroceo

HS $\frac{\text { FLOW CONDITION }}{\left(F_{\text {syst }}\right)}$

High

Medium

Low

High

Medium

Low $\frac{\text { PWL (duct) }}{\left(\mathrm{db} \text { re } 10^{-13}\right.} \frac{\text { PWL (horn) }}{\text { watts) }}$

115 *

111

116

$105 *$

111

116

$113 *$

$113 *$

112
$106 *$

$110 *$

112

The values with the asterisk are not in accord, the duct PWL being higher than that estimated from the horn. A major reason for this is that the measured SPI is dependent on the air flow velocity and gradient (Fig. 5.17) which is much higher in the duct on the average than in the termination. We might postulate that the true fan noise is really that measured in the quiescent regions of Fig. $5.17 a, b$ left side, and the rest is due to air turbulence within the duct. There is insufficient evidence to support this however because:

1. The PWL of the fan runners alone as measured in the anechoic room would exceed the fan PWL estimated as intimated above. The runner PWL readings may be somewhat high because of the low frequency directivity factor being greater than unity due to the muner location as shown in Fig. 3.3. p.97. The inadequacy of the anechoic room gave runner PWL information which established the order of importance of the blade turbulence noise, but did not give it precisely.

2. The unknown behaviour of the windscreen in certain turbulent conditions as mentioned in connection with the upper curves of Fig. 5.16.

3. There are significant line components $r_{1}$ and $r_{2}$ in the 
20 - 75 c.p.s. band below horn cut-off. These may result in a higher measured PWL in the duct than in the termination, for reasons given in Art. 2.1 .

The evidence does remain nonetheless that the higher the gradient of velocity in the air stream beyond the horn mouth, the higher is the SPI in the quiescent regions of the termination, fan speed being constant. For flow densities such as represented by the lower right map of Fig. $5.17 \mathrm{a}$, one might expect the noise generated by this turbulence and its reaction with the bounding walls to be nearly of the same order as the noise from the fan runner blade turbulence itself, which is the main source of noise in the system. If for example a constricting throat such as that used in supersonic wind tunnels is inserted in the system, it is not unreasonable to expect a significant noise increase, solely due to air turbulence and the reaction on it of the bounding walls.

It is instructive now to examine the noise spectra in the flows of Fig. 5.17a, b and compare them with Mawardi and Dyer's 36 wind tunnel noise spectra. (Ref.Art.1.2). The spectra are plotted in Fig. 5.18a, b. The stations referred to in Fig. 18 are found by the points of intersection of the appropriately numbered arrows on the perimeter of the SPL contour maps, Fig. 5.17. To avoid overcrowding of Fig. 18, the legend refers to the curves by the plotted point symbols and type of line (eg. whether solid, dotted, etc.). Observe the following (referring to Fig. 5.17 and 5.18 ): 


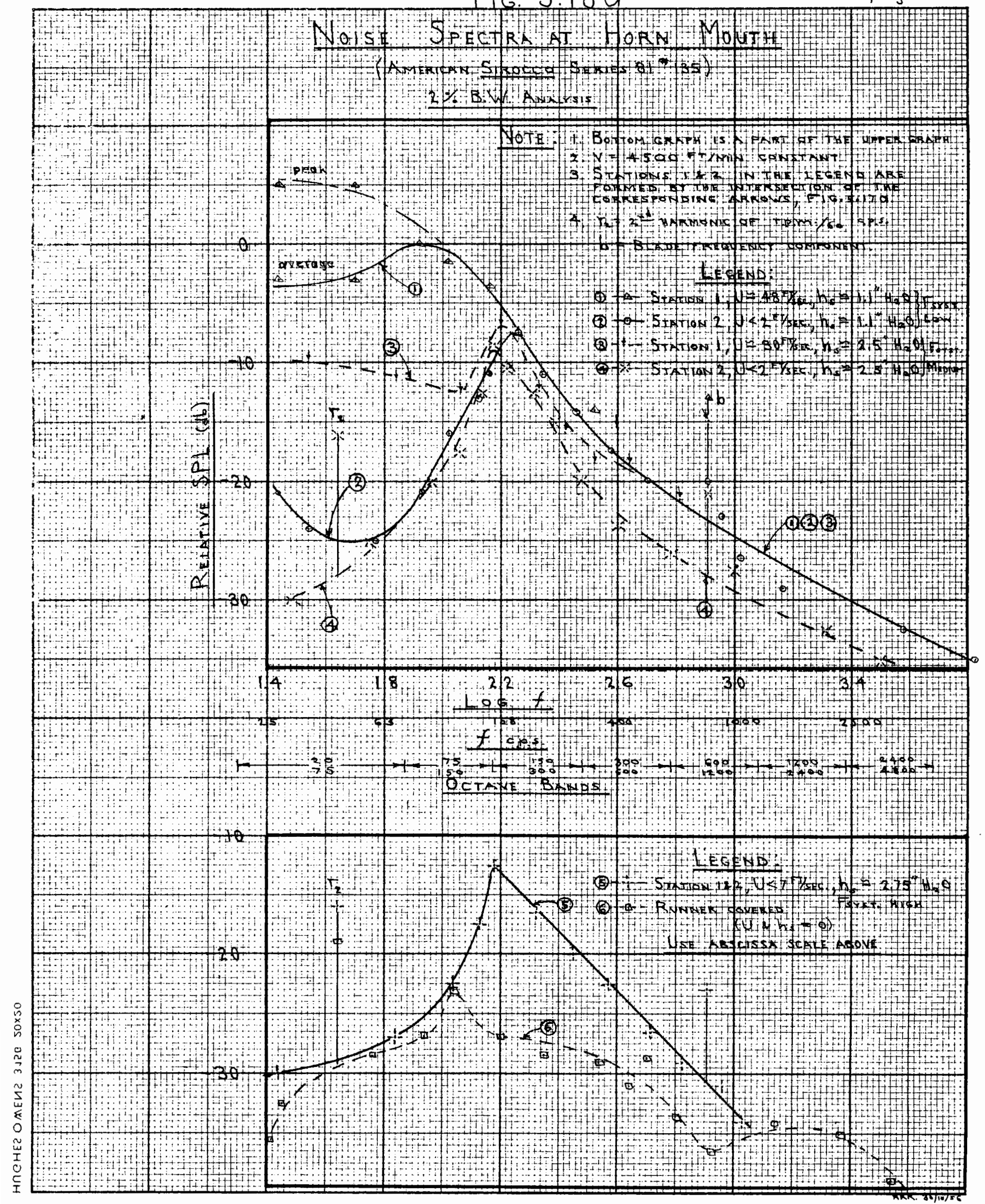




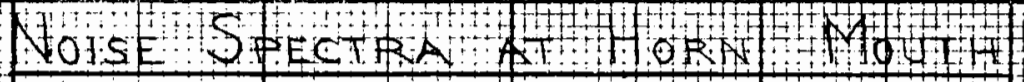

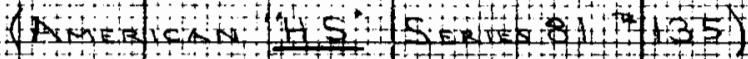

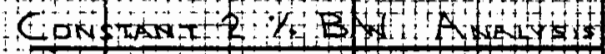

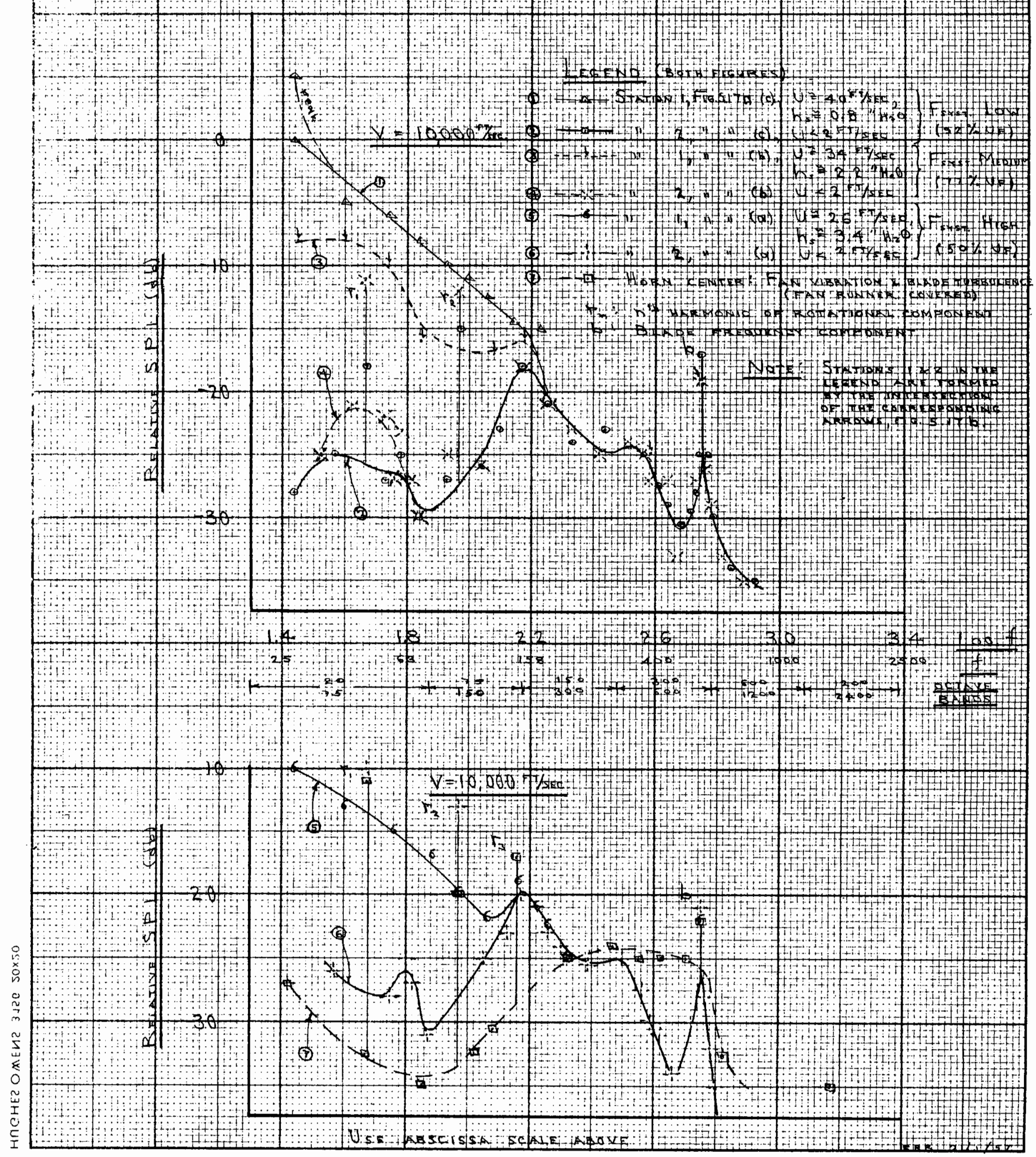


Fig. $5.18 \mathrm{c}$

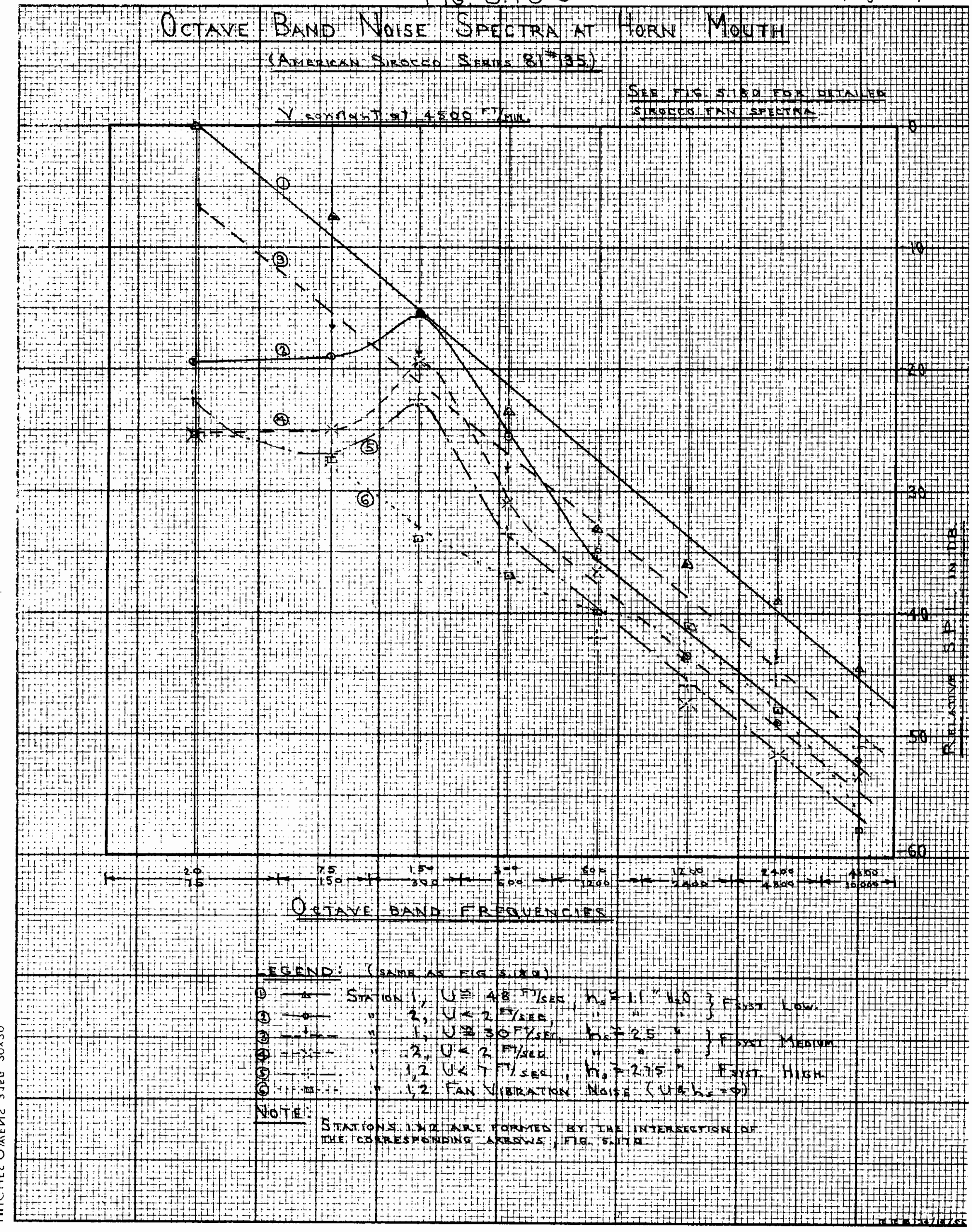


1. The similarity in spectra shape of the Sirocco and HS fans for equivalent $F_{\text {syst }}$ and flow condition.

2. The rather minor rôle of the blade passage frequency and adjacent spectrum for the HS $\mathrm{fan}$, compared to the prominence exhibited by Fig. 5.16b.

3. The significant hump in the spectra stradding the 120 to 180 c.p.s. band. This hump is apparent upon examination of the fan outlet noise in the duct, Fig. 5.13. There it grew in magnitude with increasing flow (decreasing back pressure) at constant fan speed. This hump is not due to fan runner turbulence or vibration, as seen froin Fig. 5.16 and the bottom dashed curves Fig. 5.18, which is the termination noise measured when the inner periphery of the runner was covered as discussed before. In this condition the runner can rotate and cause some air turbulence at the blade tips, but cannot deliver air (no pressure differential across the blades). The spectrum becomes much flatter in this region as fan speed, hence flow decrease; and becomes more pronounced as speed and flow increase. This part of the spectrum is attributed to aerodynamic noise caused by the combined action of turbulent air flow, and termination and horn boundaries reacting on the turbulence. These boundaries are "soft" since they are composed of I" fiberglas on quite rigid wood panels. For a given fan speed, a higher flow causes a wider, higher maximum than low flow, high back pressure. The low frequency side of the hump especially reflects this.

4. The vast increase in low frequency noise when the microphone is placed in a high speed, concentrated flow compared to a 
lower speed distributed flow, or compared to placing the microphone in a quiescent region (study Fig. 5.17, 5.18 carefully). This phenomenon is similar to that encountered in the anechoic room runner tests, when the windscreen covered microphone was placed in the turbulence. Some of the increased signal is undoubtedly due to increased noise, but some of it must also be due to the design and presence of the windscreen covered microphone in the flow, as discussed in connection with the upper curves of Fig. 5.16, and al so in Art. 3. If this additional signal was entirely turbulence noise, then the low frequency portion of the spectra, Fig. 5.18 taken in the low flow regions of Fig. 5.17, would be higher.

The conclusion that the 120 to 180 c.p.s. (approx.) maxima in Fig. 5.18 are due to aerodynamic noise (point 3 above) is substantiated by the following theoretical and experimental evidence :

(I) Plotting the spectrum level of this region against $f / f \max$. ( $f \max$ being the frequency of maximum signal) allows comparison in Fig. 5.19 with Mawardi and Dyer's 36 wind tunnel noise spectra. As pointed out in Art. 1.2, their work was done on supersonic flows which differ aerodynamically from subsonic flows, but in which the mechanism of noise generation is comparable. They conclude that their spectra definitely point to noise of aerodynamic origin. Results were compared with Mawardi and Dyer's only for the Sirocco fan, but similar results obtain for the HS fan, as comparison of Fig. 
359.61G KEUFFEL a ESSER CO.
Semi-Loyarithmic. 2 CyCles $\times 10$ to the inch

th lines accented

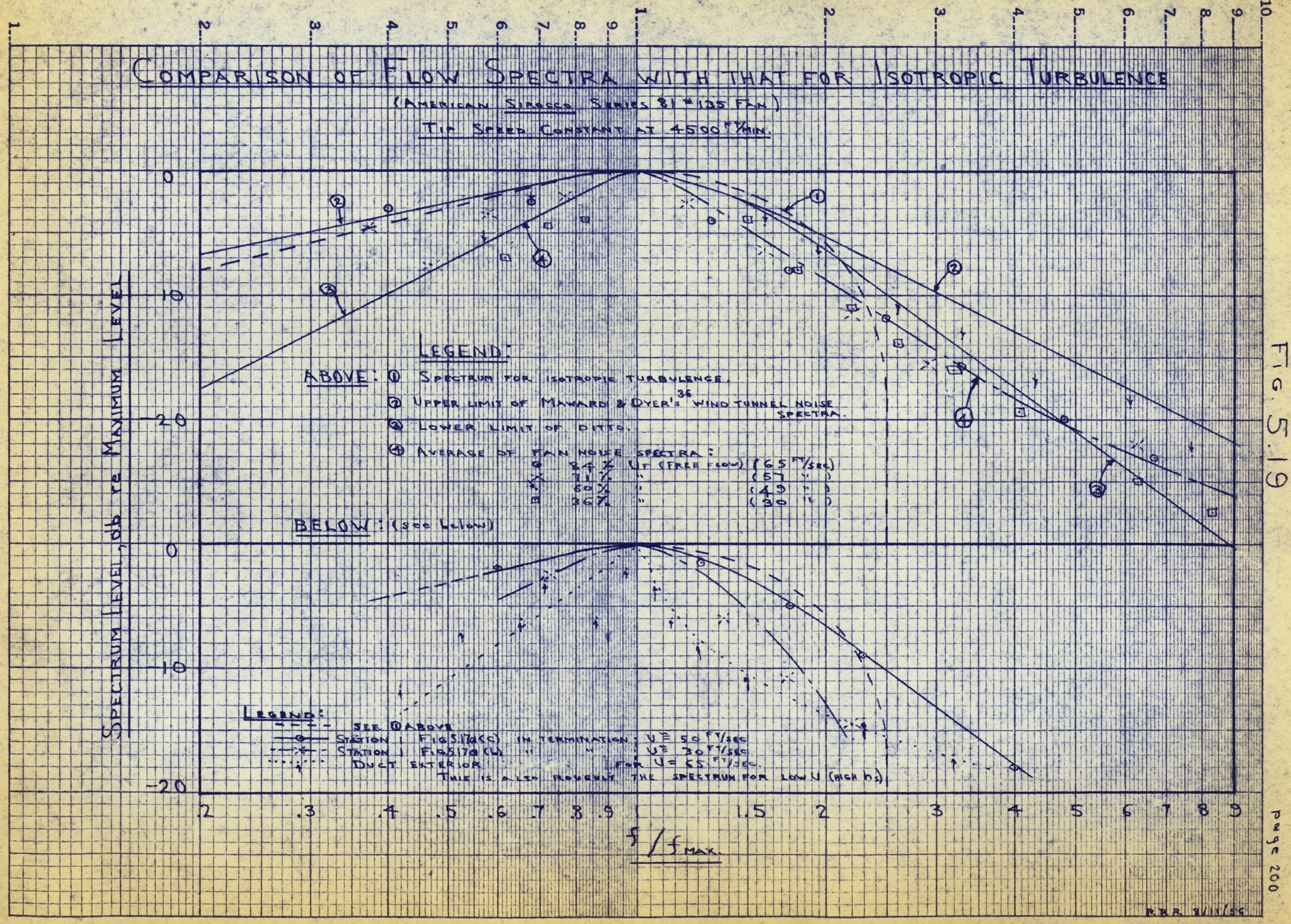


5.18a and $b$ reveals. The higher the speed and concentration of the flow, the closer the noise spectrum measured in the quiescent region near the flow approaches that of Mawardi and Dyer's wind tunnel noise, and also that for isotropic turbulence 27 .

(2) The higher the Mach number of the flow, the greater the noise magnitude and the higher the maximum frequency 18,44. This is evident in Fig. 5.18a where the solid curve, low graph, is for the lowest flow, (Fsyst high Fig. 5.17a), the- $\therefore$-- curve, upper graph is for a higher flow (Fsyst medium Fig. 5.17a) and the curve is for the highest flow (F syst low Fig. 5.17a). Note how the hump widens, shifts and increases in magnitude as flow velocity increases.

It appears that most of the low frequency signal increase when placing the windscreen covered microphone directly in the flow is not true fan - flow noise, as this would be contrary to the theory that suggests that as flow increases, so also does the frequency of maximum signal. Generally the spectrum seems to be a maximum near the lower audible limit, decreasing with frequency. There is only one shred of experimental evidence to the contrary, as seen from comparing the low frequency portion of the-- - $-\ldots+\ldots$ and - - $-\infty-$ curves Fig.5.18 (both lower and upper graphs). These curves correspond to a microphone position in the maximum flows of Fig.5.17 for the appropriate $F_{\text {syst }}$. Comparing the three curves, the lowest is defficient of appreciable low frequency contribution $(U \max =30 \mathrm{ft} / \mathrm{sec})$. The time average of the highest flow 
(U $\max =48 \mathrm{ft} / \mathrm{sec}$ ) shows a maximum near 85 c.p.s. This growth and shift of the low frequency contribution to the spectrum vaguely fulfills theoretical expectations. It may simply be due to a new scale of pressure fluctuations superposed on the flow turbulence, caused by the presence of the windscreen covered microphone.

If what has been said is true, then at higher flows, the low frequency portion of the noise spectrum as measured in the duct may be high. This would account for the increase of I with $V^{x}$ or $U^{x}$ when $x$ becomes increasingly greater at high flows for the Sirocco fan (Fig.5.8a). It would not account for the uniform exponent obtained with HS fan, because the power of the $r_{1}, r_{2}$ rotational line component is relatively great when compared with the adjacent continuous spectrum. The error cannot be very large however because octave bandwise the outlet noise spectra as measured in the duct retain their relative shape at various fan speeds for constant percent free flow reasonably well, except for the 75 c.p.s. to 300 c.p.s. bands which increase proportionately with flow. This is a result of the increased turbulence noise discussed above in connection with Fig. 5.17, 5.18, 5.19. The bandwise increase of noise with speed is left to Art. 5.2.5.

Fig. $5.18 \mathrm{c}$ is the octave band equivalent of Fig.5.18a. 


\section{$\underline{5.2 .4 \quad \text { VIBRATION }}$}

Classified under this heading are the following noise sources:

1. The rotational line components $r_{1}, r_{2}$ etc. as previously defined, due to runner unbalance and finite flexibility of fan base and housing.

2. Miscellaneous sources of vibration coming from the $f$ an plates, bearings, etc. Duct vibration was confirmed negligible by measurement.

Most of the graphical data is for the Sirocco fan. Where results differ appreciable for the HS fan, they have also been included. The reader is referred to Art. 4.1 .4 for method of measuring fan vibration.

When the wavelength of sound emitted from a vibrating source is larger than the source dimensions, acceleration (acc.) of the source is the best criterion to assess probable sound output. When the wavelength of emitted sound is smaller than the source dimensions, velocity (vel.) of the source is the best criterion. Since the tested fan principal dimension is on the order of one foot, the approximate division between acc. and vel. is 1000 c.p.s. Nearly all the vibration spectrum occurs below this, thus acceleration is the prime indication of probable sound emission.

As mentioned before, the closest attempt at measuring noise due to vibration was by covering the inner runner periphery so that the runner could not deliver air. However when the runner rotatea, considerable turbulence was still in evidence 
at the blade tips, masking noise due to vibration. Some correlation was obtained by comparing the spectrum of this noise with that due to runner turbulence Fig. 5.16 and acceleration of the fan housing, as described in Art.4.2.3. The comparison of covered runner noise and $f$ an housing acceleration is made in Fig. 5.20: (Sirocco $f a n$ ) and 5.22. (HS fan). The seeming jumble of curves on the lower half of Fig. 5.20 are for four conditions of fan operation at constant speed. Except for details, they are similar. Compare the general trend of these curves with the noise spectrum curve obtained for the covered runner (microphone in the duct). In Fig. 5.22, the two curves to compare are 1 and 2. The acceleration spectrum, curve 2, for the HS fan changes but little with varying fan operation at constant speed. The general similarity between the noise and acceleration curves at some of the frequencies indicates that part of the noise is due to fan vibration. Where correlation is not good, the major noise is likely due to turbulence at the fan runner blade tips. In other words, for the sirocco runner, most of the measured noise from 25 to $400 \mathrm{c.p.s}$. is due to vibration. For the HS runner, line components $r_{1}, r_{2}, r_{3}$ and continuous spectrum from about 200 to 400 c.p.s. is attributable to fan vibration. The upper graph of Fig.5.30 shows the overall Sirocco vibration noise to be at least 10 to $15 \mathrm{db}$ below the overall $f$ an noise. The upper graph of Fig. 5.15b shows the overall HS noise to be only 3 to $5 \mathrm{db}$ below the overall fan noise. This is primarily due to the large rotational line components $r_{1}, r_{2}$ and $r_{3}$. Were it not for these, the vibration noise would be 10 or more $d b$ below the overall noise. The magnitude of the rotational components is a function of 


\section{Fan Vibration and Vibration Noise Spectra.}

$$
\frac{\text { Constant } 2 \% \text { B.W Anmeysis }}{\text { (AmERICAN Sirocco Series } 81 \text { (35) }}
$$

$10-90+0^{-3} \quad \frac{\text { TIP SPEED CONSTANT AT } 4500 \mathrm{rT} / \mathrm{MIN}}{4}$
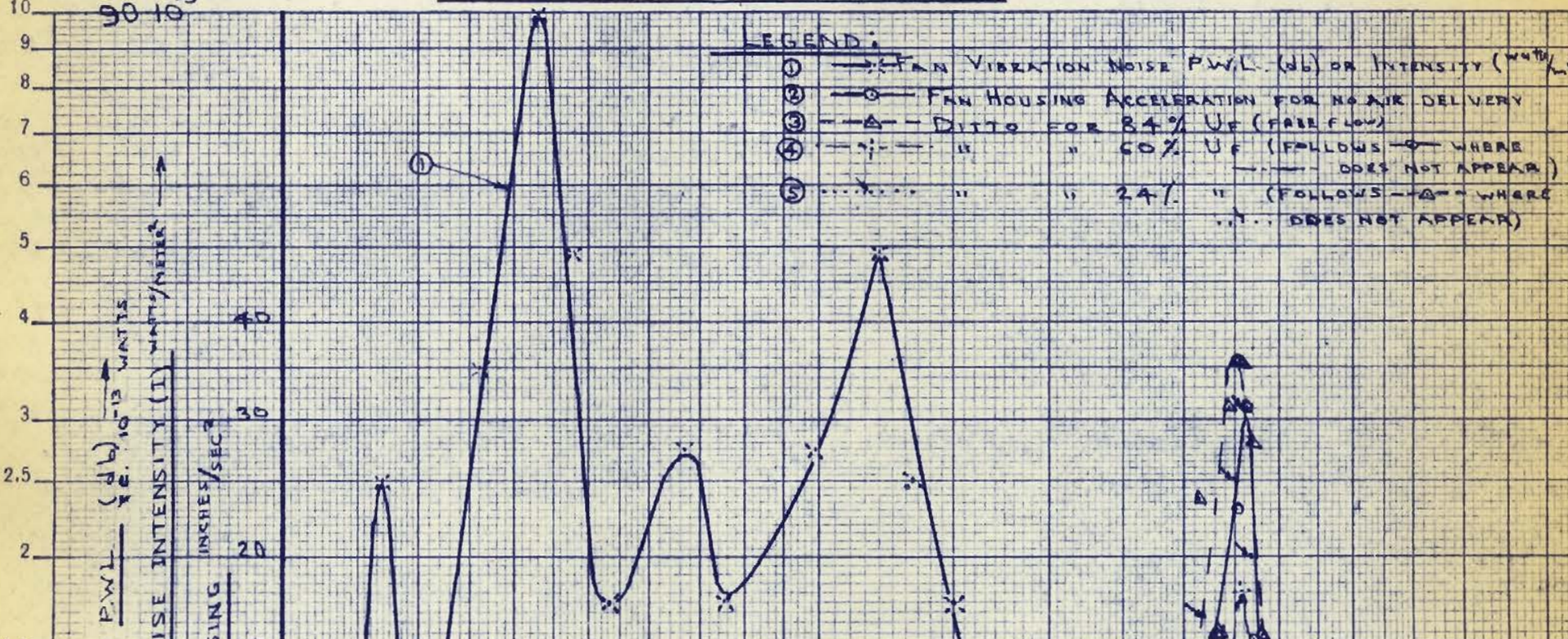

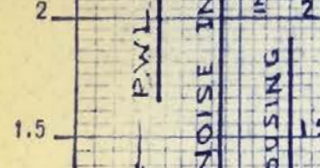

$1^{\frac{2}{3}}$,

을

oi $\frac{1}{4}$

or 4

4

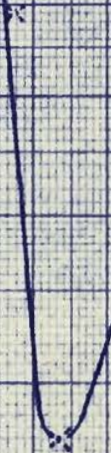

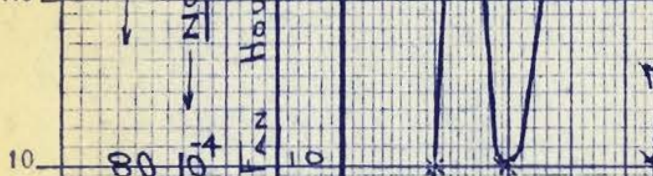

$r_{2}=\frac{\operatorname{spm}}{60} x^{2}$

$\int_{0}$

\begin{tabular}{l|l|l}
9 & 80 \\
\hline 8 & 8
\end{tabular}

\begin{tabular}{r|r|l}
\hline & 2 & 2 \\
\hline & 7 & 6 \\
\hline & 2 &
\end{tabular}

$\mathrm{j}_{4}$

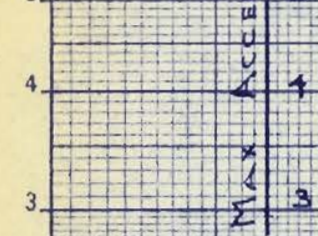

2.5

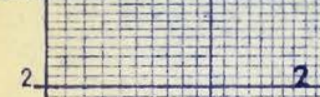

1.5

$\left.\frac{7010^{-5}}{1.5}\right|_{20} 2.530$
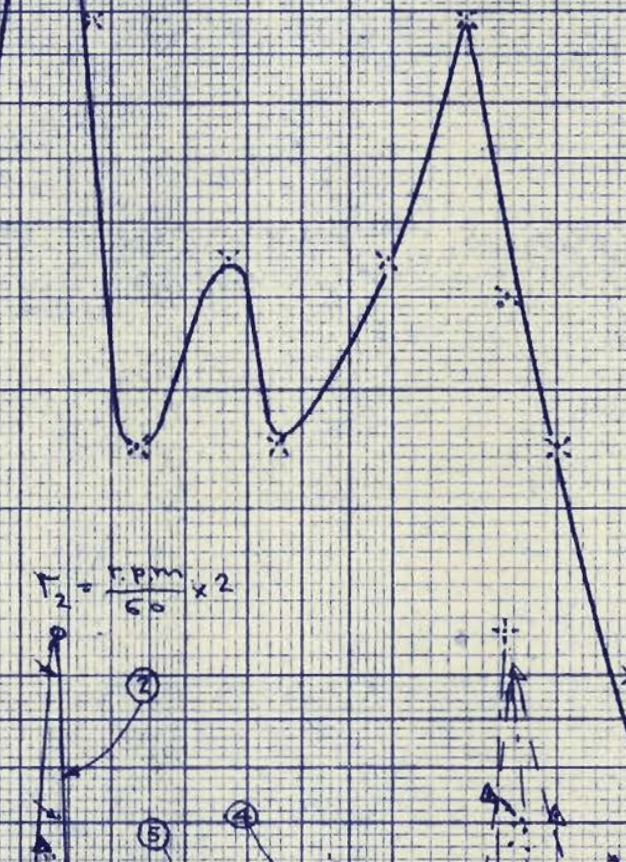

冓
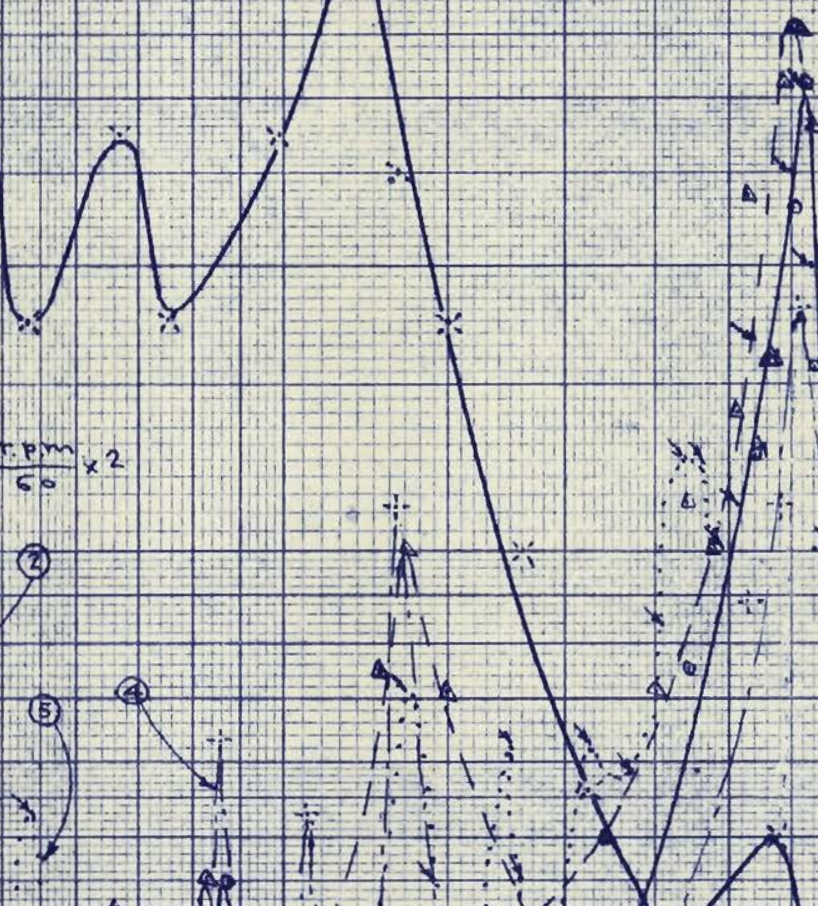


$$
\frac{\text { Fig. } 5.21}{\text { Fan VELOCITy VIBRATION SPECTRA }}
$$

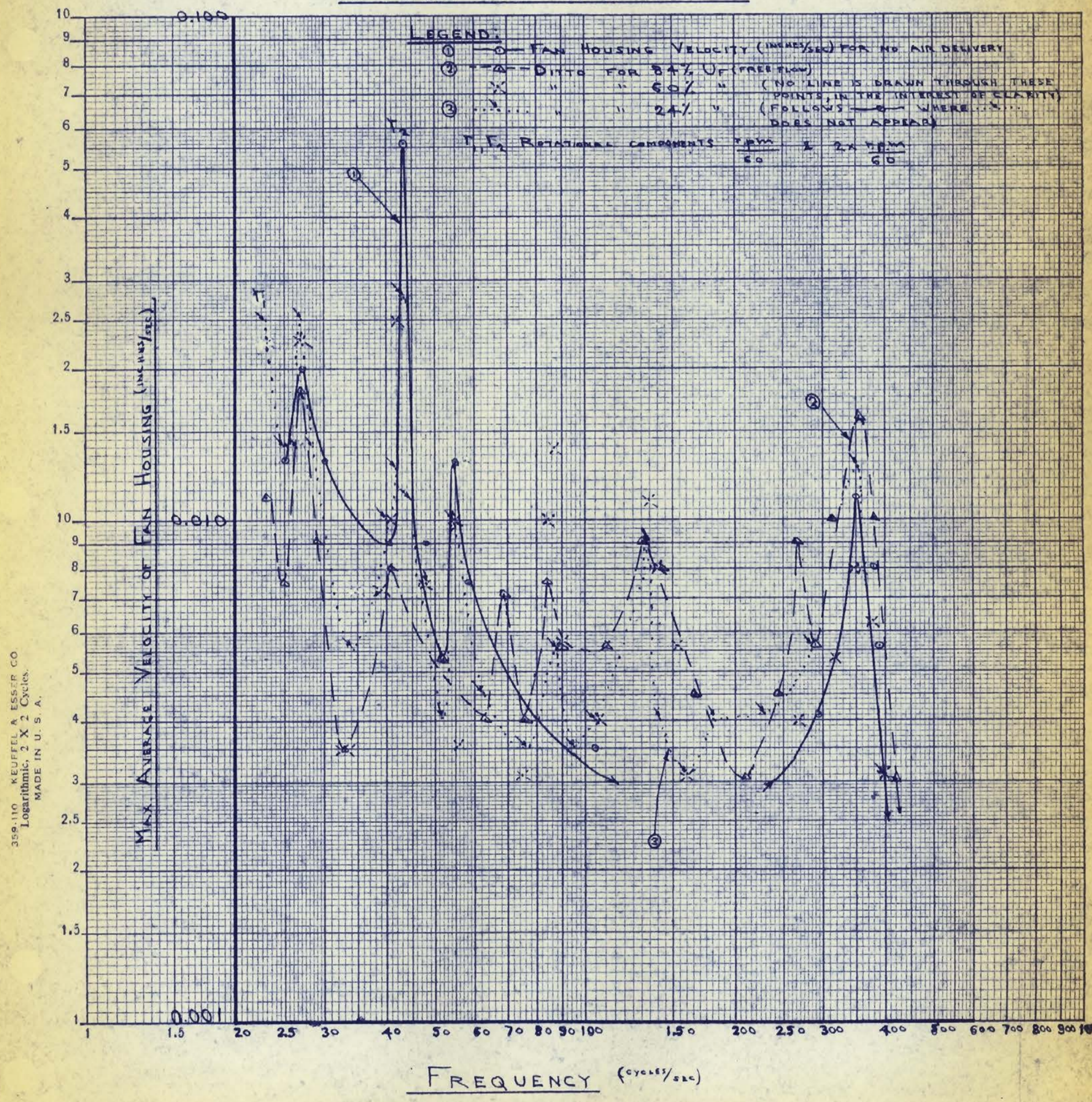


F io. 5.22

page 207

$\frac{\text { Fan Vibration and Vibration- Blade Turbulence Noise Spectra }}{\text { Constant } \% \text { B. Whatisis }}$ (American "HS" Series 81 "135)

Tip Speed Constant at $10,000 \mathrm{Ft} / \mathrm{min}$

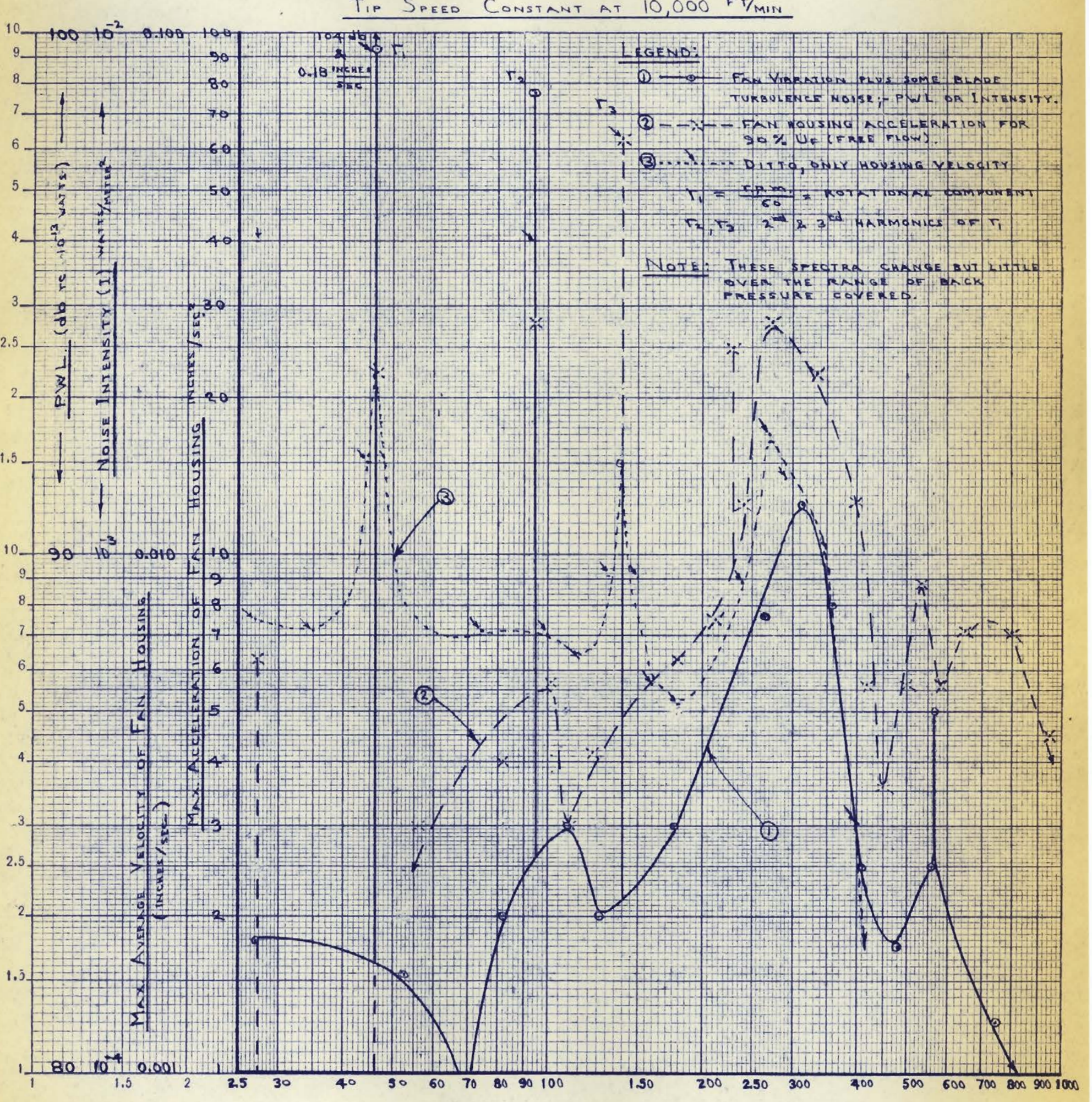

Frequency (crales/sec) 
dynamic runner unbalance, fan rigidity, installation rigidity and rotor speed. The effect of the fan base is covered in Art. 5.3.2.

Curve 3, Fig. 5.22 and Fig. 5.21 give vibration velocity spectra for the HS and Sirocco fans respectively. As predicted, the spectral correlation between sound and vibration velocity becomes more positive with increasing frequency. Again Fig. 5.21 contains three velocity curves, all similar, differing slightly with various conditions of sirocco fan operation at constant speed.

Fig. 5.23 illustrates how little the bandwise acceleration of the sirocco fan changes with percent free flow at constant speed. The three solid curves prove that increasing the speed results in a greater change of overall fan vibration with percent free flow with constant speed.

Overall vibration acceleration and velocity increase with fan speed is plotted in Fig. 5.24 for the Sirocco fan. Results are similar for the HS fan. The dashed envelopes represent the total scatter of points for the various speeds. This scatter increases with speed and is due to the $V$ - curve effect noted in Fig. 5.23 for various fan operation at constant speed. The higher the speed, the sharper this variation. The minimum vibration occurs at maximum mechanical efficiency (near maximum static efficiency). These variations are not nearly as great for the HS fan. Observe that as speed drops, fan acceleration and vibration approach a relatively constant low value and the scattering of points decreases. Actually, the average vibration should decrease quite linearly (aside from structural resonances) 


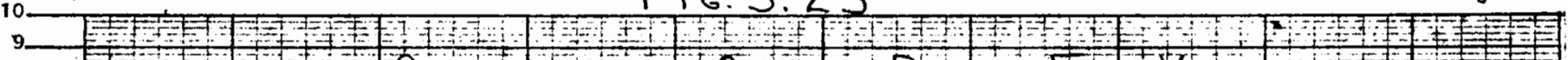

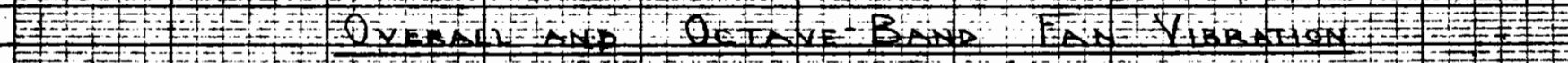

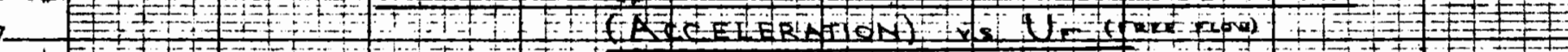

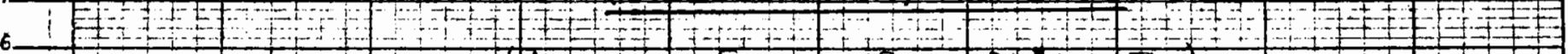

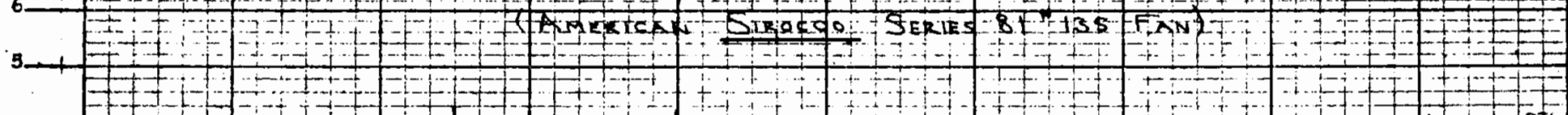

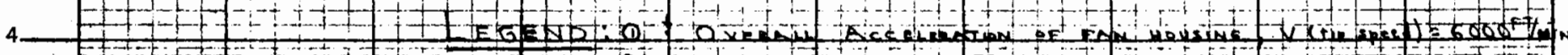

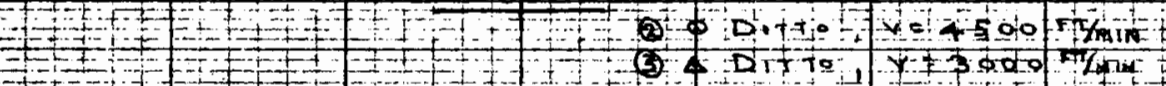

$$
3 .
$$
$e_{1}=$

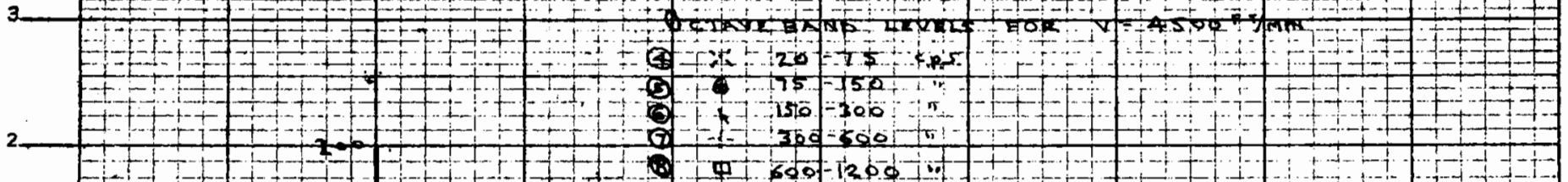

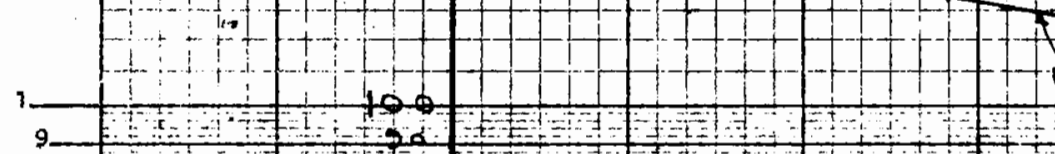
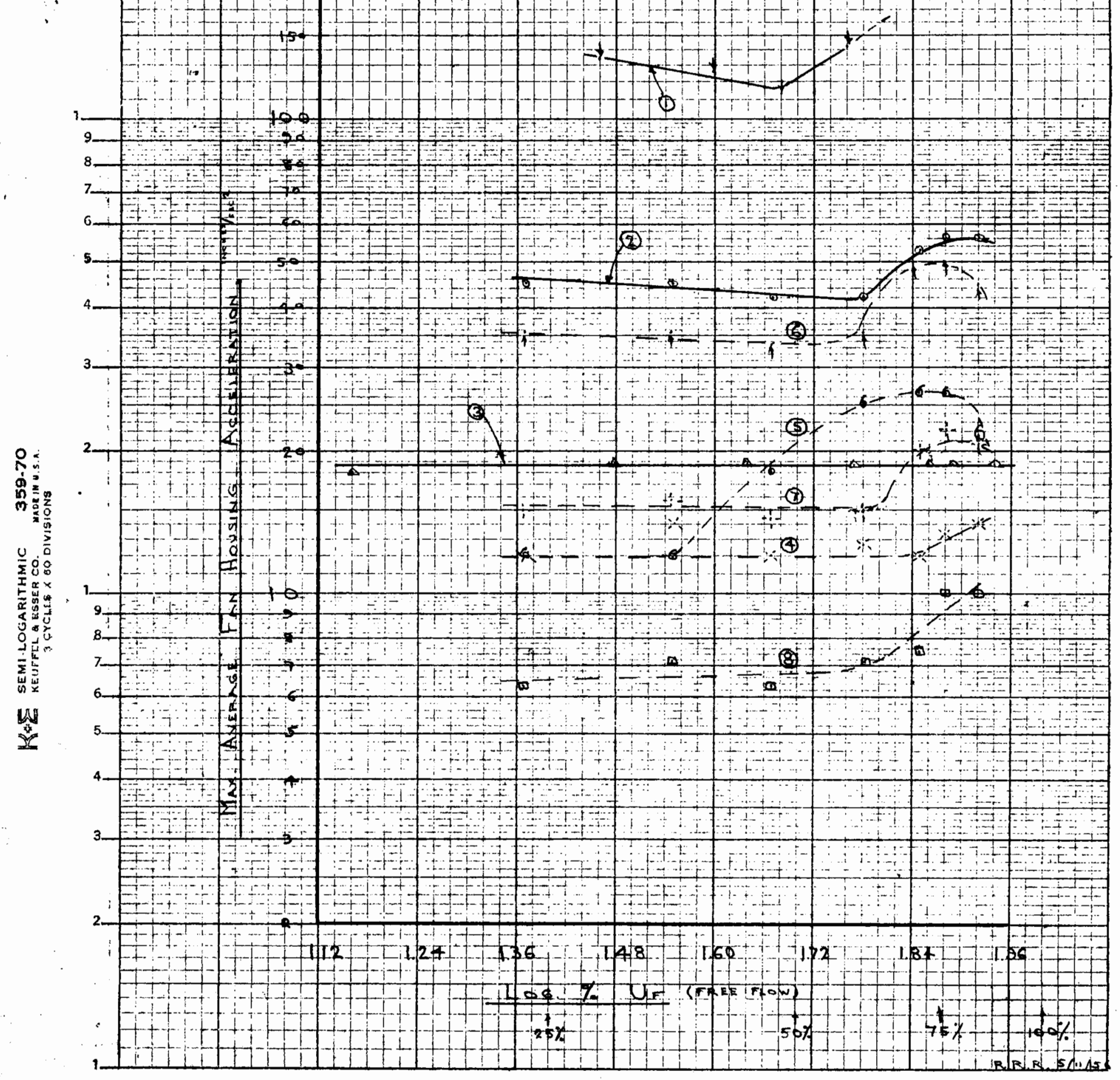


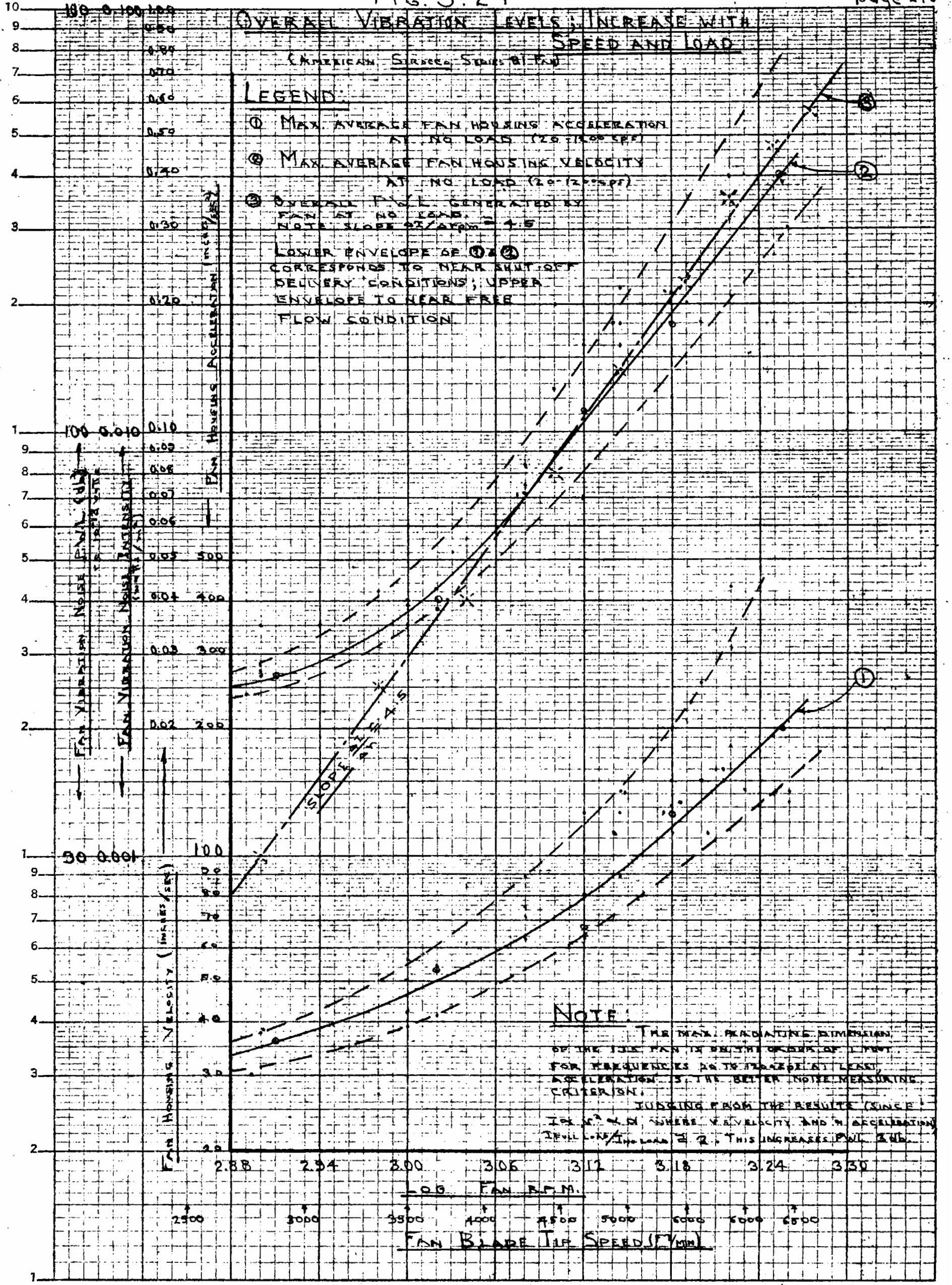


with speed, approaching zero as speed does. The change in slope with increasing speed is a consequence of aerodynamic reaction on the fan. The fan is now able to deliver useable quantities of air at significant pressures. The straight line of slope $\frac{\Delta I}{\Delta n}=4.5$, Fig. 5.24 is the increase of sound intensity in the duct due to the covered sirocco runner (mainly vibration noise) with runner speed. Near top speed, $\frac{\Delta V e l}{\Delta h} \cong 4.5$;

$\frac{\Delta \text { Accel. }}{\Delta n} \cong 4$. Sound pressure $p \propto$ r.m.s. velocity of the vibrating source $(v)$. But acceleration $a=\frac{d v}{d t}$. Since sound intensity $I \propto p^{2}$, it also varies as $v^{2}$ or a. Considering the final average slope of velocity and acceleration with fan speed, it is apparent that it is the acceleration which primarily determines $\Delta I / \Delta$ r.p.m. This was predicted earlier. The solid curves within the envelopes of Fig. 5.24 are for the covered fan runner (no air delivery or "no load" as stated on the graph). The upper envelope as a ratio with this no-load curve represents a ratio of about 2 . Since. I $\alpha$ a, the increase in $f$ an vibration PWL between "no load" and the worst condition (near free flow) is about $3 \mathrm{db}$ at the high speeds. Thus it is possible to estimate the probable range of vibration noise for different ranges of back pressure at constant speed. This has been done to obtain the envelope on either side of the solid curve, upper graph of Fig. 5.30. This spread is much less for the HS $f a n$, hence has not been computed for Fig. 5.15b upper graph.

The final vibration graph, Fig.5.25a,b is a bandwise plot of acceleration with speed. The solid lines are for $F_{\text {syst }}$

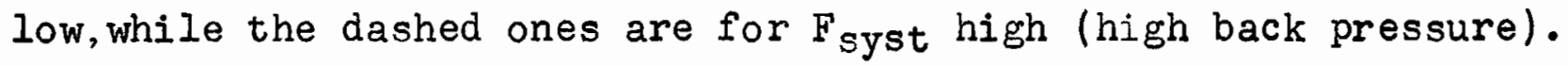
The solid curves are not as extended because of lack of driving 


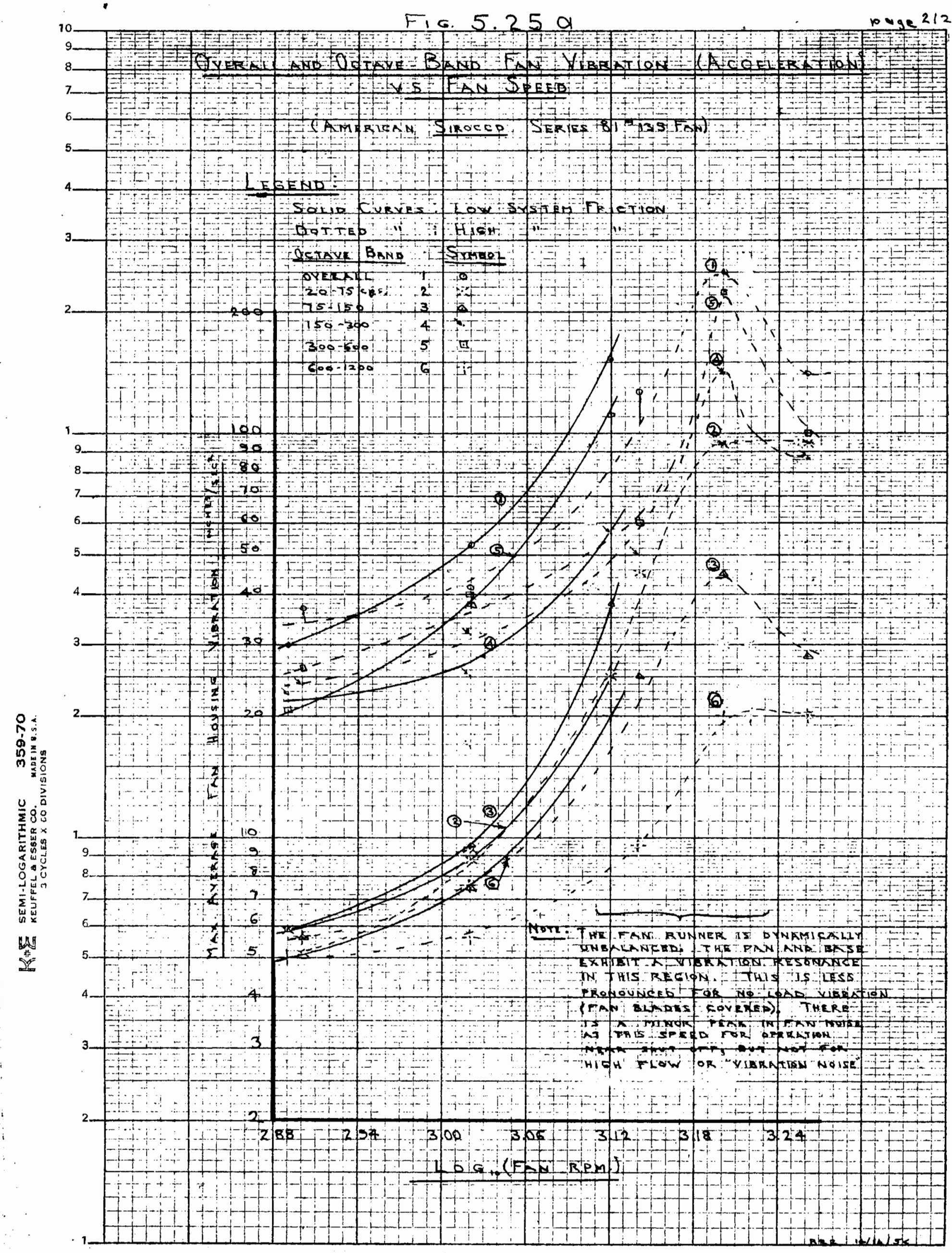


FIG $5.25 \mathrm{~b}$

page 213

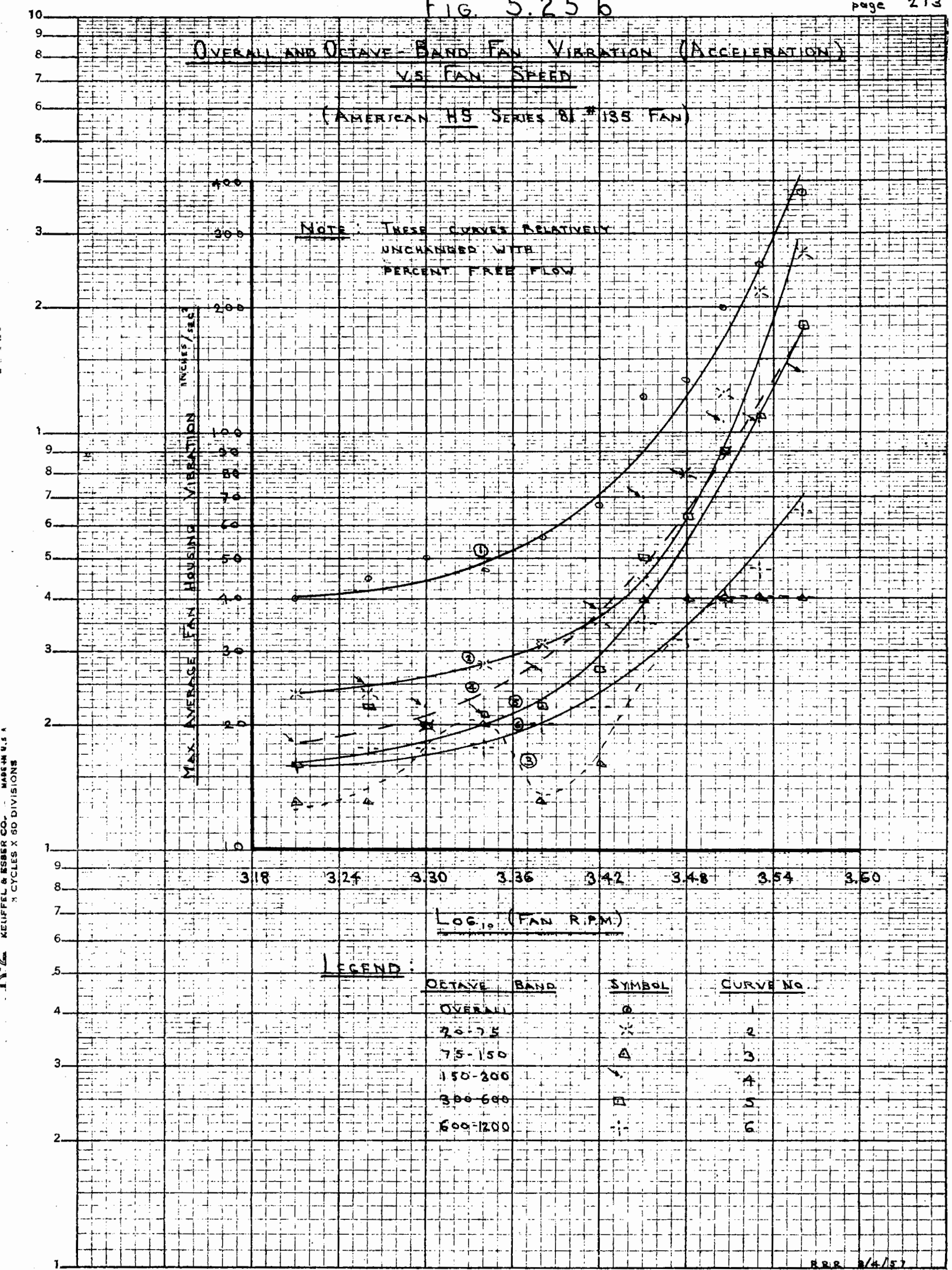


motor power. The results indicate that the acceleration of the Sirocco fan does not increase indefinitely at an increasing rate as indicated in Fig. 5.24a, but appears to pass through a resonance condition. Note too that most of the vibration is in the lower two bands. The HS fan results show a rapid increase of acceleration similar to the Sirocco fan, then a tapering off. There is a suggestion of installed fan resonance near a fan speed of $1800 \mathrm{r.p.m}$. in Fig. 5.25b for the $F_{\text {syst }}$ high condition, but not the $F_{\text {syst }}$ low. This is probably a consequence of the back pressure reacting aerodynamically on the runner blades to increase the reciprocating thrust on the fan housing at its resonant point (near $30 \mathrm{c.p.s.}$ ). Base resonance is discussed in Art. 5.3 .2 .

5.2.5 OCTAVE BAND FAN NOISE INCREASE WITH SPEED

We have come to valuable conclusions from the foregoing fan noise spectra, Art. 5.2.1 to 5.2.4, regarding the origin of $f a n$ noise in particular. It has more or less been established that the main noise sources are from air turbulence at the blades and in the duct (or at the fan outlet flange if the duct area is much greater than $f a n$ outlet area), plus line rotational components due to fan runner unbalance causing vibrations and "pumping" of air as outlined in Art. 2.2. There is still another avenue worth exploring to confirm these conclusions, and that is to observe how the various noise sources we have measured increase bandwise with fan speed.

Octave band fan outlet noise increase with fan speed is given in Fig. 5.26a, b for widely different flow conditions. 
Fig. 5.26 a

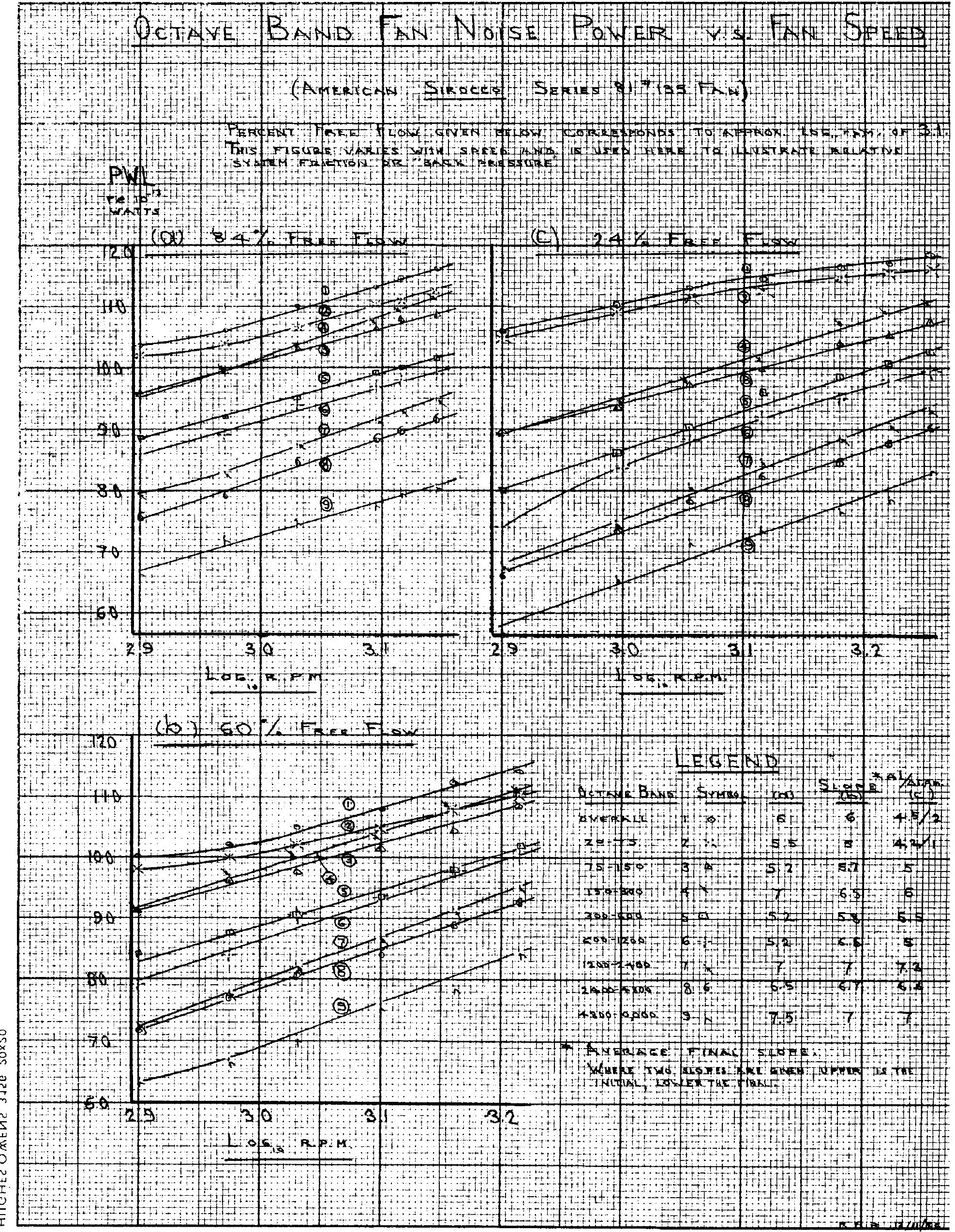




\section{Fis. $5.26 b$}

page 216

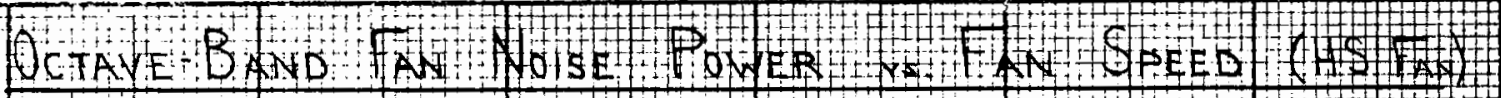

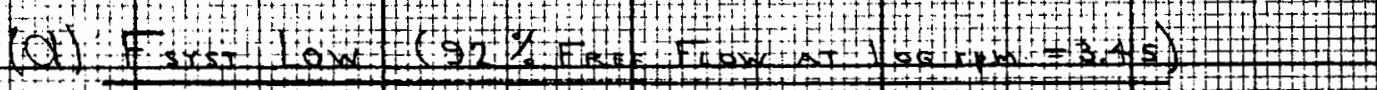

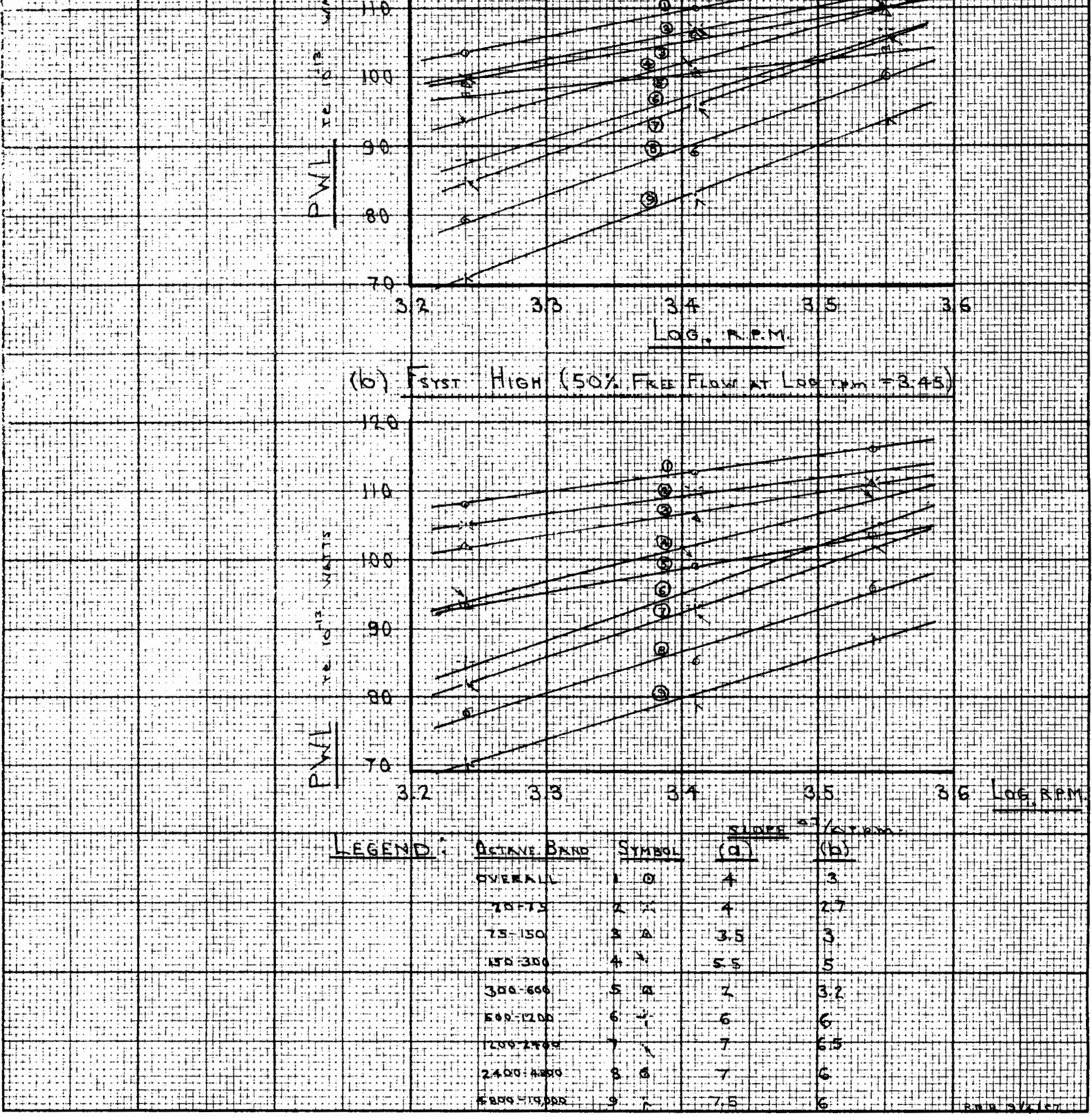


The desired information may be hard to pinpoint for the uninitiated due to the large amount of data condensed onto these graphs. Aid is given by referring to the legend on each page where the slope of $\Delta I / \Delta r . p . m$. is listed for each band and flow condition, and each curve is referred to by the plotted point symbol and a number. Two observations should be noted on the part of the reader on this type of graph; the slope of the appropriate band vs. speed, and the relative position of the octave band curve with respect to the overall noise curve. In Fig. 5.26a for the sirocco fan, note the relative importance of $3^{\text {rd }}$ and $4^{\text {th }}$ band (where the overall audio band is here termed the $1^{\text {st }}$ band) noise for flow conditions (a) and (b) compared to (c). These bands contain most of the aerodynamic noise generated by air turbulence in the duct. As such they should increase with about the $6^{\text {th }}$ power of fan speed 34 . It is seen this is approximated for the sirocco fan, and only for band 4 in the case of the HS fan, Fig. 5.26b. In addition observe:

1. The lower slopes of the first 5 bands for the HS fan compared to the Sirocco fan.

This is attibutable partly to the blade turbulence noise, as shall be seen, and partly to the relative importance in the HS fan noise of rotational components $r_{1}, r_{2}, r_{3}$ caused by runner unbalance, hence vibration. Correlation will also be seen with this later.

2. The absolute value of most of the bands is somewhat higher for the HS fan than the Sirocco, keeping in mind that for approximately equivalent operation the HS runner operates at twice the Sirocco speed (eg. add 0.3 to the Sirocco abscissa scale). This is a consequence of the higher opera- 
tional speed, hence larger blade turbulence noise.

3. The higher the air flow (eg. the higher the percent free flow or the lower the back pressure) for a given speed, the higher is the magnitude of the upper bands of noise, and also the faster they increase with speed. This is a combination of blade and duct turbulence. It is seen from Fig. 20 that there is negligible vibration in the upper three bands but that in Fig. 5.19 aerodynamic noise is very wideband. The higher the fan speeds and air flows involved, the higher the significant noise extends into the upper frequency regions.

4. Second band noise is most important in low $\% U_{F}$ sirocco fan noise. This is possibly due to a modifying effect of back pressure on blade turbulence and increased $r_{1}$, Fig. $5.13 a$.

5. The difference in results between high $\% \mathrm{U}_{F}$ and low $\% \mathrm{U}$ is more marked for the Sirocco than the HS fan (remember this was true for overall fan noise PWL). Graph (a) and (c) Fig. 5.26a corresponds to the same Fsyst as (a) and (b) respectively in Fig. 5.26b.

Before making definite conclusions, let us examine Fig. 5.27 and 5.28. Fig. 5.27 contains the bandwise increase with speed of noise generated by the "covered" Sirocco and HS fan muners (Art. 5.2.4). The noise is due to vibration and some turbulence at the blade tips. Note:

1. The slopes of bands 20 to $600 \mathrm{c.p.s}$. have decreased compared to Fig. 5.26. Especially observe this reduction for the 150 to 300 c.p.s. band where aerodynamic noise was 


\section{Fig. 5.27}

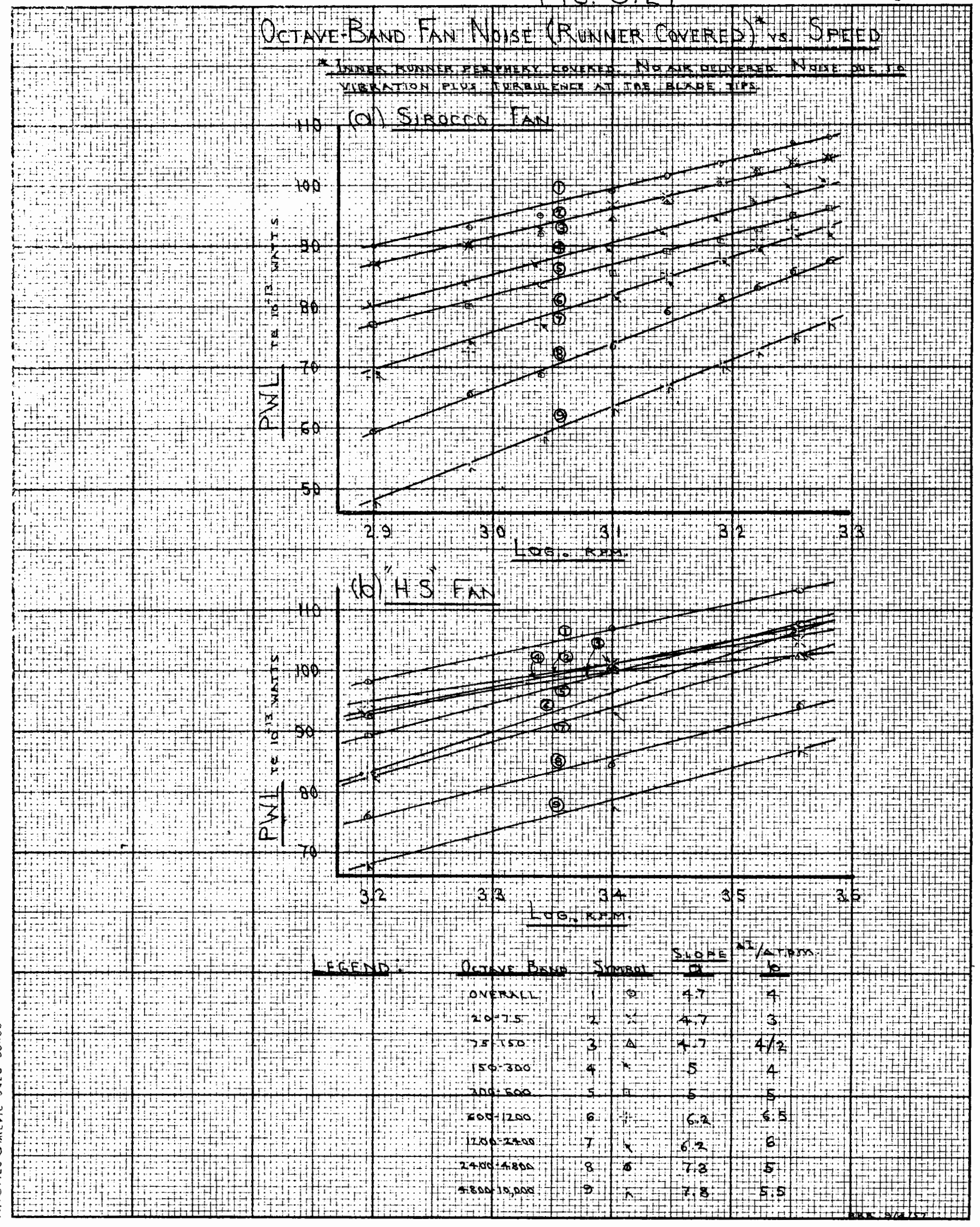


most evident before.

2. All levels are higher for the HS fan due to the added vibration of higher speed operation and added blade turbulence.

3. The 300 to 600 band becomes the major contributor at the highest HS muner speeds. This is the band of maximum blade turbulence power, Fig. 5.16b.

Fig. 5.28 contains two important sets of data; octave band increase of runner noise with speed, and the effect of octave band levels and increase in speed due to placing the microphone in a very turbulent stream. For partsakb, the runner noise exhibits the following characteristics:

1. The important bands increase with speed to the $6^{\text {th }}$ and $7^{\text {th }}$ powers, as boundary turbulence noise should according to the theory.

2. If $\mathrm{n}$ is Sirocco runner speed and $2 \mathrm{n}$ is the equivalent $\mathrm{HS}$ runner speed, the overall HS runner turbulence noise exceeds that of the sirocco by about $5 \mathrm{db}$.

3. The band following the 300 to $600 \mathrm{c} . \mathrm{p.s}$. band in which maximum HS runner noise exists (see Fig.5.16b) contains more power due to the higher average level and double the frequency range (eg.600 to 1200 c.p.s.).

4. Bands 2, 3 and 4 of the HS runner noise contain mainly vibration noise from the assembly in the anechoic chamber. They are of minor significance at the higher speeds. Band 2 (dotted) contains a resonance (runner support resonance). Parts cad are bandwise comparisons of noise in Station 1-1, Fig. 5.17 a bottom, and Station $2-2$ of the same figure. The first 


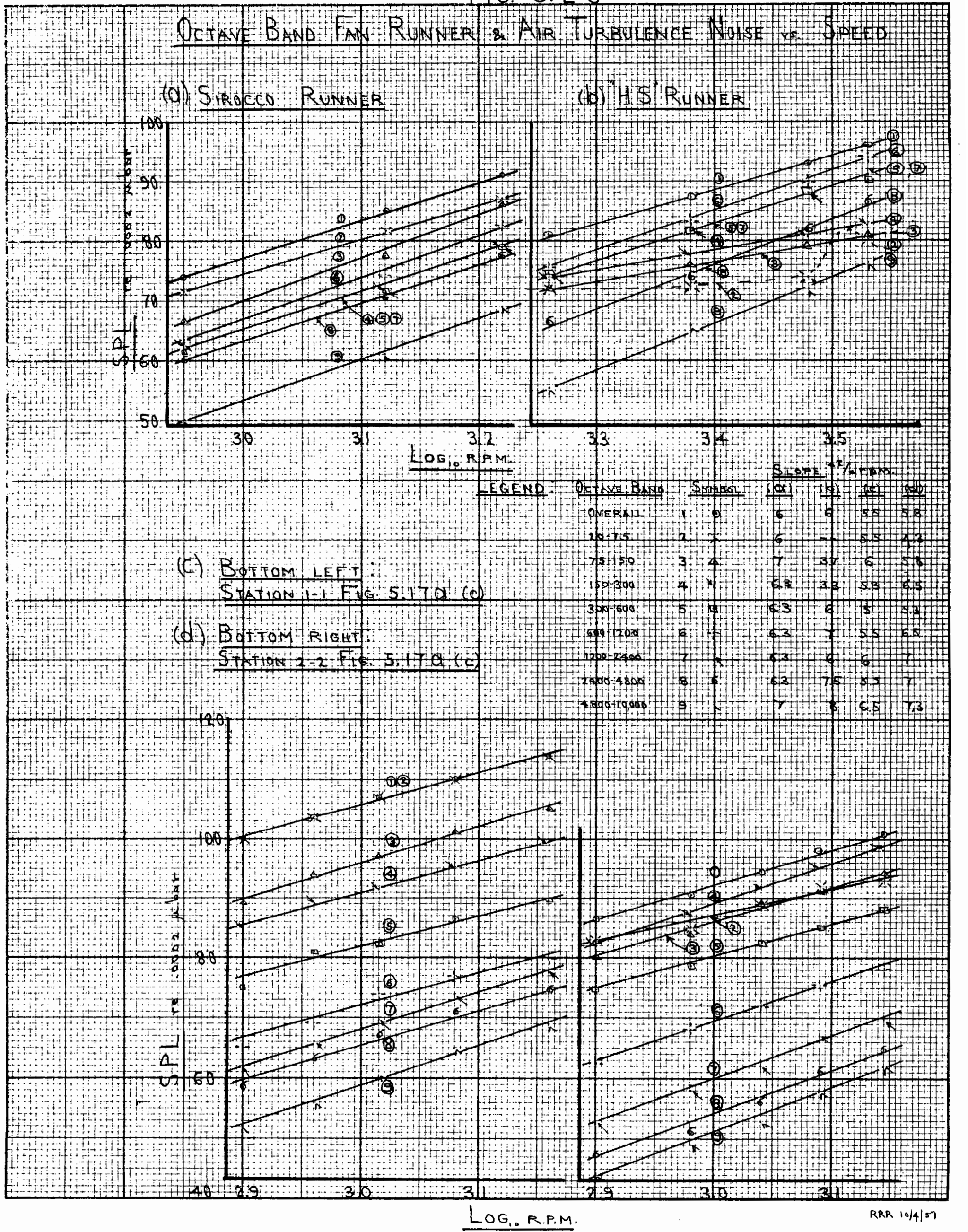


graph in the cid part of Fig. 5.28 is for the maximum flow position, the second graph for the quiescent flow adjacent to the main turbulent stream. Note particularly that bands, 1, 2, 3, 7, 8, 9 increase appreciably due to the microphone being placed in the flow. In both cases the slopes of all the bands increase with speed to the $5^{\text {th }}$ to $7^{\text {th }}$ power, except the $20-75$ c.p.s. band in the quiescent flow which increases with the $4^{\text {th }}$ power. Essentially all of the power within the flow is in the $20-75$ c.p.s. band as confirmed in Art. 5.2.3.

The conclusions to be drawn from the results of this section are:

1. Fan muner and stream turbulence noise verify the theory that they are of boundary turbulence origin thus increase with the $6^{\text {th }}$ power of speed.

2. The upper three bands (1200 to 10,000 c.p.s.) are predominantly aerodynamic noise as suggested by theory, the results of Fig. 5.19, and the lack of significant vibration towards this band. (It was not possible to accurately measure vibration above about $1000 \mathrm{c} . \mathrm{p} . \mathrm{s}$. because of nonlinear response of the vibration measuring system; ref.63 p.8). The fan noise spectra behave similarly in this range.

3. Those bands which fail to increase with the $5^{\text {th }}$ power (or greater) of speed are probably influenced by vibration noise; in particular the rotational component $r_{1}$ and its harmonics. For fan operation below maximum static efficiency $\left(\eta_{s} \max .\right)$ this becomes increasingly apparent for the lower bands of noise. Consider the slopes of the fan 
outlet noise bands with speed for high $F_{\text {syst; }}$ (c) Fig. 5.26a and (b) Fig. 5.26b for the sirocco and HS fans respectively. In the $20-75 \mathrm{c} . \mathrm{p} . \mathrm{s}$. band for the sirocco and the 20 to $150 \mathrm{c.p.s}$. band for the HS fan, rotational components $r_{1}, r_{2}, r_{3}$ are known to become an appreciable portion of the noise in these bands. These line components increase with fan speed at a rate which is characteristic of the fan - base vibration (resonance effects, etc; see Fig. 5.25a,b). The 300 to $600 \mathrm{c.p} . \mathrm{s}$. band varies but little with the HS fan speed. Evidence of $f a n$ vibration and runner noise increase with speed in this band points to a $5^{\text {th }}$ power relation instead of 3 as obtained. It may be explained by the gradual shift of important components with speed. For example in Fig. 5.22, there are two significant components in this range. One is a maximum near 310 c.p.s. and the other is the "blade" component at 565 c.p.s. at the particular blade tip speed of $10,000 \mathrm{ft} / \mathrm{min}$ (2830 r.p.m.). At lower speeds, the rather broad maximum is in the 150 300 c.p.s. band while the blade component is within the 300 - 600 c.p.s. band. At higher speeds the first maximum is in the $300-600$ c.p.s. band while the blade component is in the 600 to $1200 \mathrm{c.p.s}$. band. At $2830 \mathrm{r.p.m}$. as plotted, they are $2 / 3$ in, $1 / 3$ out of the band. The effect is to raise the band power at low speeds due mainly to vibration, decrease it at high speeds due mainly to a shift in the major part of the blade turbulence power out of the band. 


\subsection{ADDITIONAL RELEVANT STUDIES}

\subsubsection{INLET NOISE SPECTRA AND BANDWISE SPEED INCREASE}

In Art. 5.1.3 fan outlet and inlet PWL were advantageously compared. There is little similarity in the spectra between ducted outlet and open inlet centrifugal fan noise. Octave band analyses for three varying flow conditions appear back in Fig. 5.14a for the sirocco fan, 5.14b for the HS fan. The three flow conditions in the legend occur for the same relative back panel (Fsyst) position for each fan. The inlet spectra appear to increase in magnitude rather uniformly with air flow (speed is constant in this graph), regardless of back pressure at the outlet. For this reason correlation between inlet and outlet PWL Fig. 5.4 was not too positive below the max. $\eta_{\text {, }}$ point (higher hs, lower $U$ ). The blade component for the HS fan is about 510 c.p.s. This accounts for the peak of the 300 - 600 c.p.s. band and indicates that the runner spectrum, Fig. 5.16b, plays a dominant role in output noise. As such we would expect most of the inlet noise bands to increase as the $5^{\text {th }}$ or $6^{\text {th }}$ power of speed. This is approximately true for all of the Sirocco fan bands and most of the HS bands as seen in the following table: 
Fig 5.29

page 226

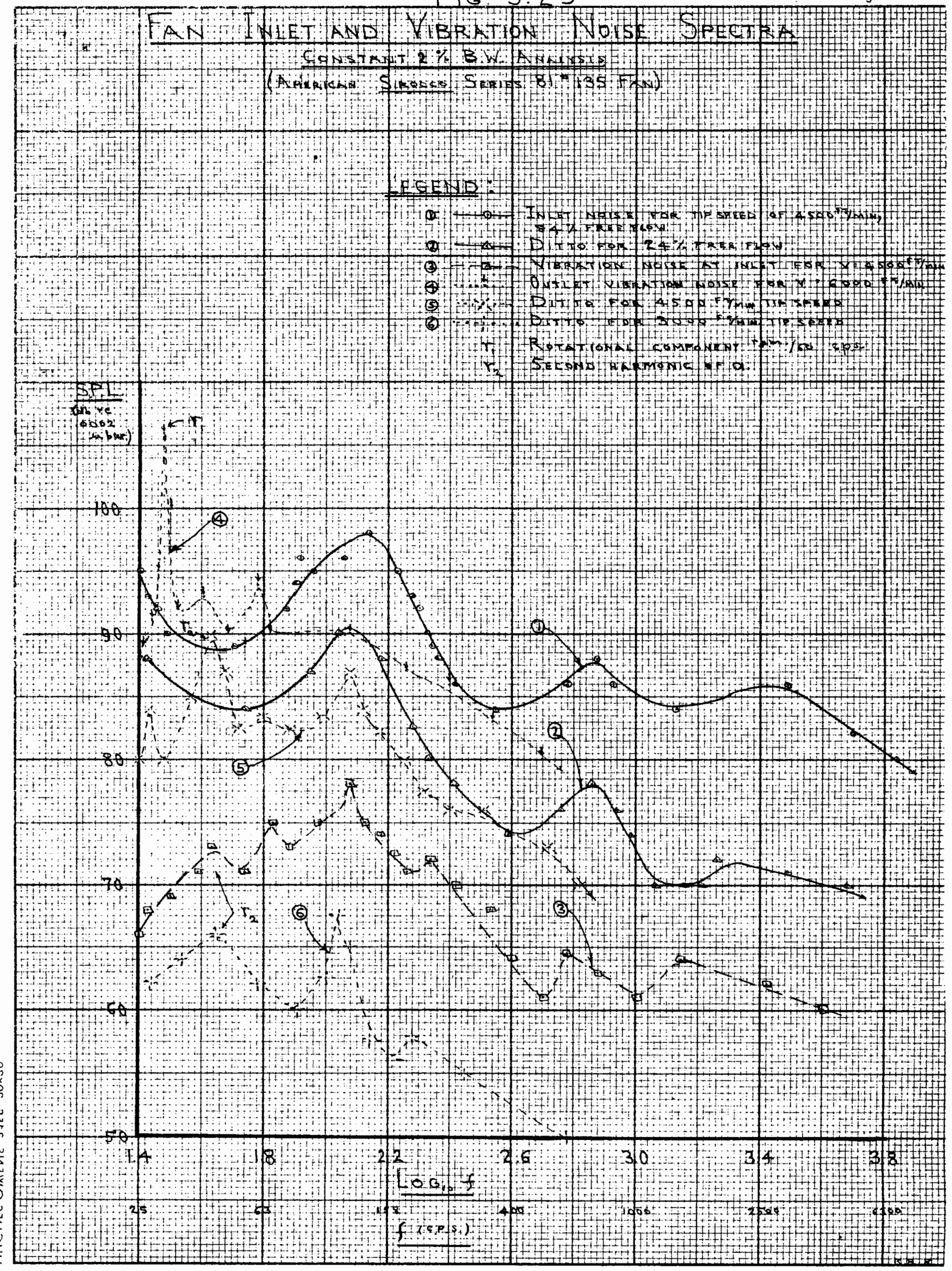


TABLE 5.2

BANDWISE INCREASE OF FAN INLET NOISE WITH SPEED $(\Delta I / \Delta n)$ BAND

SIROCCO

F syst Low Fsyst High Fsyst Low Fsyst High

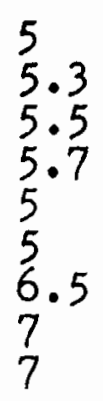

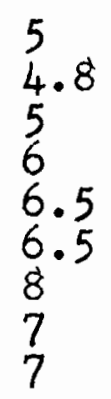

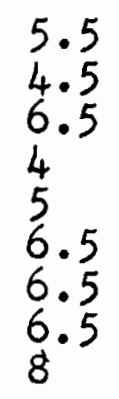

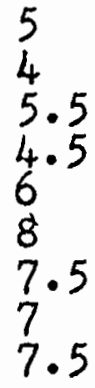

A $2 \%$ B.W. spectrum for the sirocco fan is given in Fig. 5.29 and for the HS fan, bottom of Fig. 5.13b. Looking first at Fig. 5.29 curves 1,2 and 3 (curves 4 to 6 were discussed in Art. 5.2.4) we find:

1. There is considerable power at the lowest frequencies. This seems due to a scale of turbulence caused by the inrushing air around the microphone windscreen plus the noise of the incoming eddies breaking on the runner. When the runner is covered for no air delivery at the same speed (curve 3), the low frequencies are well down.

2. In curve 3 there is a vibration maximum centered at 120 c.p.s. (see also Fig. 5.20). Note how this increases in magnitude, width, and how it shifts to the right slightly as air flow increases at the same speed. This is due to both added magnitude of vibration and air turbulence in the flow, mainly the latter as pointed out in Art.5.2.3.

3. There is a second small naximum in curves $I$ and 2 centered at the blade passage frequency, $570 \mathrm{c.p} . \mathrm{s}$. There is a falling spectrum of some width on either side of this blade frequency, accounting for the broadness of the maxi- 
mum as contrasted with a line component. The blade spectrum appears more significant in the inlet noise than the out let noise.

4. Note the marked decrease in noise as back pressure increases (flow decreased) at the same speed. Curve 2 also decreases more rapidly at the higher frequencies, as was found in the outlet spectra Fig. 5.13a.

The HS fan inlet noise spectra are plotted in the 10wer half of Fig. 5.13b for the same two Fsyst as used for curves $I$ and 2 Fig. 5.29. The spectra are quite different from the Sirocco spectra, the line components and spectrum associated with the blade passage (the maximum centered at b) playing the dominant rôle. The $n^{\text {th }}$ harmonic rotational components are $r_{1}, r_{2}$ and $r_{3}$. The decrease in power with decreased flow at constant fan speed does not lower the inlet spectrum significantly except for frequencies above about 300 c.p.s.

\subsubsection{FAN INSTALLATION VS. NOISE}

There are generally three requisites for installation of centrifugal fans for minimum noise:

1. Quiet prime mover and drive (eg. - electric motor and $V$ - belts, fluid drives).

2. A vibration break between fan and ducts to prevent transmission of vibration down them.

3. A rigid base insulated from the building as far as possible.

The original cause of vibration at the rotational fre- 
quency ( $\frac{\text { r.p.m. }}{b U}$ c.p.s.) and its harmonics is runner unbalance. This mechanism of generating noise was discussed in Art.2.2.4. Runner eccentricity on the order of 0.010 " for the sirocco fan, $0.005^{\prime \prime}$ for the HS fan was noted using a dial micrometer (having multiplied travel) on the inner runner periphery. The cast hub for the sirocco fan was visibly eccentric. The runners had been factory balanced however as evidenced by drilled holes and/or welded counterweights. Dynamic unbalance and structural flexibility of the fan supports is very critical as far as vibration and rotational line components of noise are concerned as witnessed by Fig. 5.20;22 and curves 1, 2 and 3, bottom of Fig. 5.30. In Fig. 5.30b, the importance of base flexibility or vibration freedom is illustrated. Curve $I$ is the vibration noise measured in the duct for the covered sirocco runner. The spectrum of this noise and the fan housing vibration is given in Fig. 5.20.. The base is that of Fig. $3.4 \mathrm{a}$ p.104; structural steel bolted to a wood-on-concrete floor. Curve 3, which lies some $4 \mathrm{db}$ above curve $I$ is for the base illustrated in Fig. $3.4 \mathrm{~b}$; a heavy wooden structure on felt vibration isolators. These isolators virtually eliminated vibration transmission to the floor, but allowed the base a certain freedom to vibrate, move, or rotate about its centroid due to dynamic unbalance of the fan runner. A $2 \%$ B.W. analysis showed the increase in PWL over curve 1 is solely due to rotational components $r_{1}, r_{2}$ and $r_{3}$ plus higher minor harmonics. Curve 2 is the result with the base of Fig. $3.4 \mathrm{~b}$ resting on planks instead of vibration isolators.

For research on noise from ventilating air flow turbulence, it is important to minimize vibration due to runner 


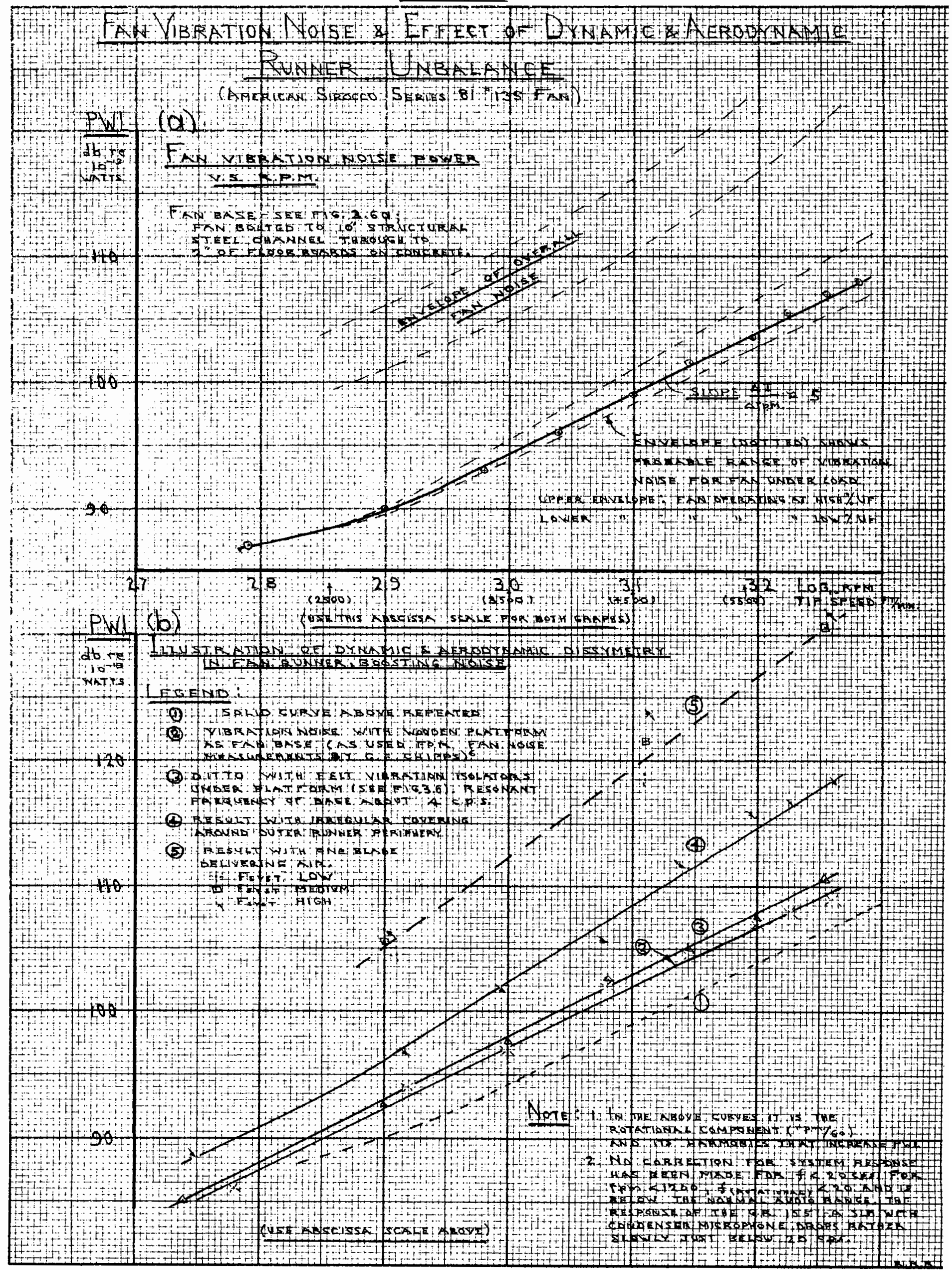


unbalance and structural flexibility if ease of analysis and accuracy of results is important. The fan used should have a well balanced runner and be mounted rigidly on a concrete base.

Large low speed fans will have their rotational fundamental well below 20 c.p.s. and harmonics near the lower audio limit. These become of negligible importance as far as the ear is concerned. They are still important when making measurements with a standard SIM or octave band analyzer because these do not cut - off sharply below $20 \mathrm{c.p.s.,} \mathrm{thus} \mathrm{it} \mathrm{is} \mathrm{possible} \mathrm{for}$ sub - audio power ta influence measured audio power in the low band.

\section{3 .3 AERODYNAMIC UNBALANCE VS. NOISE}

This refers to unsymmetrical inlet air flow, uneven blade spacing or shape and any eccentricity of the runner, not from the viewpoint of vibration it creates, but from the viewpoint of a "cam" action generating air pulses. The important manifestation of these faults is added power to the rotational line component and harmonics. Some aburd conditions were established to illustrate this point. Curve 4, Fig. $5.30 \mathrm{~b}$ is the PWL resulting from the sirocco runner when it was covered on the outside periphery. Due to centrifugal force, the cover was not perfectly concentric with the shaft and it also possessed a ridge. Although the fan delivered no air, the PWL was high because of line components $r_{n}, n=1$ to 8 , the fundamental being the largest component. Next a cover was inserted over the inner periphery of the fan such as was used for vibration noise measurements. A slot equal to one blade width $(1 / 36$ of the Sirocco runner circumference) was cut out of the covering. The 
fan could then build up some pressure across one blade only. The measured PWL ( in the duct) is given by curve 5, Fig.5.30b. Rotational harmonics up to the $10^{\text {th }}$ were measurable with the $2 \%$ B.W. analyzer. Next, 26 out of the 36 Sirocco bladeswere exposed (adjacent to one another). The measured overall PWL was:

\begin{tabular}{|c|c|c|}
\hline r.p.m. & Fsyst low & Fsyst high \\
\hline 800 & $130 \mathrm{db}$ & $123 \mathrm{db}$ \\
\hline 1200 & $139 \mathrm{db}$ & $127 \mathrm{db}$ \\
\hline
\end{tabular}

The resulting air pulses propagating down the duct were "deafening" and the fan vibration noticeably increased. These results are probably high because of the low frequency storage of energy in the duct below horn cut-off (see Art. 2.1). These are ridiculous examples of course, but they serve to illustrate that the contribution to rotational line components of noise due to slight aerodynamic unbalance is not to be ignored.

There is additional evidence in the literature (ref.51) that if there is an induced rotary motion of the inlet air in centrifugal fans of relatively few blades (radial type) particularly, the blade passage frequency noise will be increased. Further, if there is an unsymetric flow to the inlet, caused by the presence of some obstruction such as a motor support near the inlet, turbulence is established such that when it breaks on the runner blades, it may give rise to product modulations of the blade passage note. (eg. If $f=$ fan note corresponding to number of blades $\times$ r.p.s, and $f$ is the frequency of the major vorticy set up by the obstruction, there will be formed tones of $\left.f_{1}, f_{1}-f, f_{1}+f\right)$. The presence of these side bands was 
not detected for various obstructions and flow conditions in this research, but is a possibility in slower speed, fewer bladed centrifugal fans and is a likelyhood in axial fans.

\subsubsection{BLADE PROFILE VS. NOISE}

Visualize fan runner blades in any cross sectional form. If they were cylindrical or tear-drop shaped, they would be expected to rotate through the air with relatively small turbulence compared to a section such as a flat plate, broad side leading. Any irregular blade shape not approaching that of a cylinder may be considered as a blunt object generating more turbulence by its motion than that of a cylinder. We would expect a greater PWL from it than from a cylinder moving through the air at the same speed. The spectra would differ widely; quite white for the plate, peaked for the cylinder, as previously considered.

A very rudimentary study was made on three common blade profiles, sketched in Fig. 5.31a. They were fitted in turn along a horizontal duct diameter, $2^{n}$ in front of the microphone location Fig. 1.3 p.18. The duct area at this section was thereby reduced $12 \%$, which is not too serious from the point of view of air acceleration around the blade. With the Sirocco fan rotating at a blade tip speed of $4800 \mathrm{ft} / \mathrm{min}$ as source, and Fsyst low, noise measurements were made downstream from the blade. If significant noise was generated, it would add to the fan noise, increasing its spectrum. This study should be accepted with reservation, since we are using a one bladed model, stationary in a turbulent airstream, to simulate a number of 
359-5 KEUFFEL ESSER CO.

$\times 10$ to the inch.

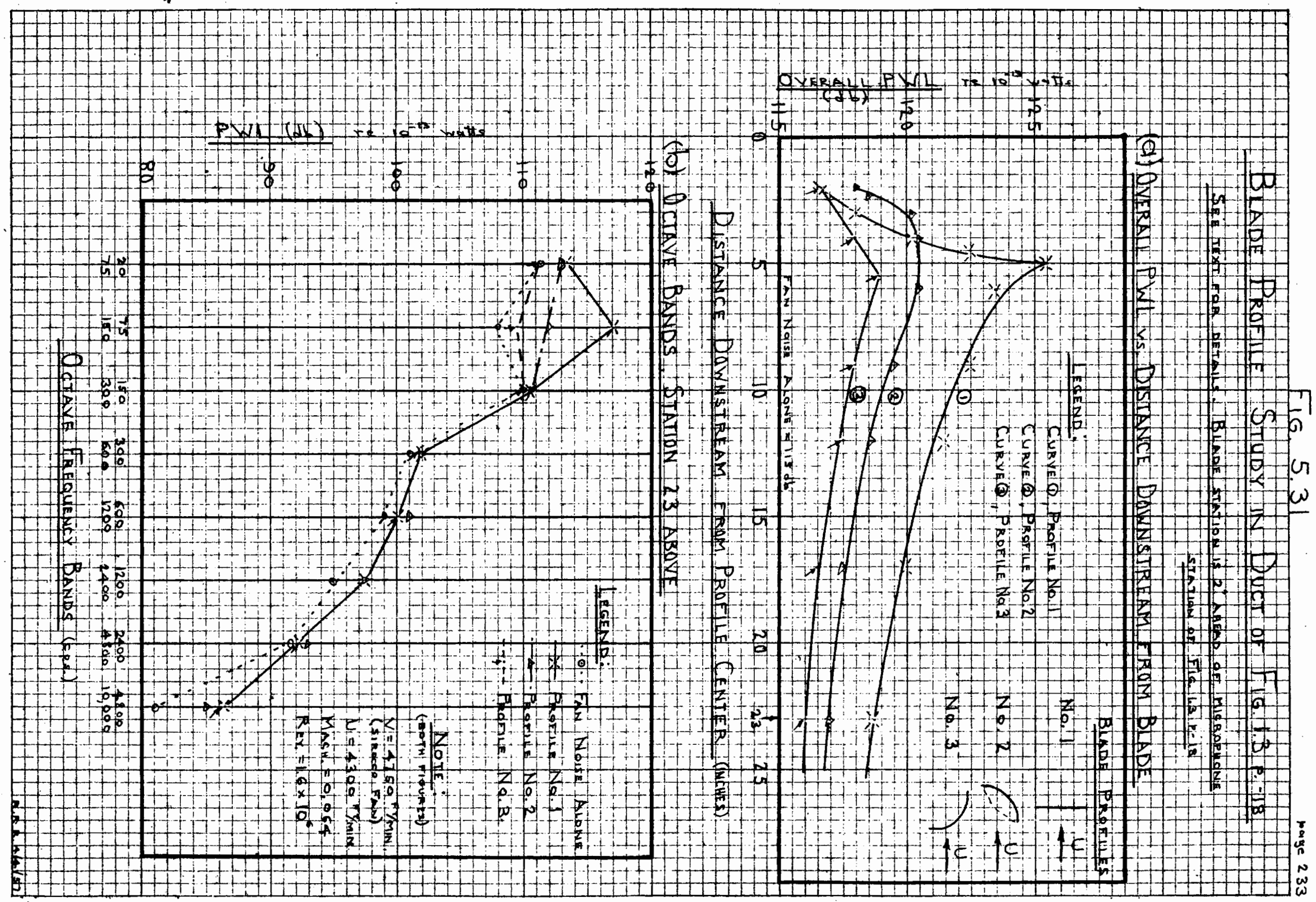


closely spaced blades rotating in a circular motion perpendicular to their length through generally quiescent air.

Fig. 5.3la shows PWL vs. traverse along the duct axis in inches downstream from the blade. This is not all noise, but is a signal produced by a scale of eddying which the microphone windscreen failed to attenuate. We learned in Art. 1.2 that at a sufficient distance downstream from a blunt object in high Rey. number flow, this eddying will quickly merge with the dominant scale of turbulence that existed prior to the object. As would be expected, the more blunt the object, the greater is the eddying following the object (curves 1,2 and 3 Fig.5.3Ia). The microphone travel limit is 23" from the blade. This is close to stabalized turbulence as seen from the final slope of the curves, Fig. 5.31a. Additional PwL that occurs at this station should therefore be due primarily to the noise generated by the blade, and not due to eddying.

Octave band spectra taken at station 23, Fig. 5.3la are shown for the three profiles, Fig. 5.31b. Note that they all add slightly to the original fan noise and that the 75 to $150 \mathrm{c} . \mathrm{p} . \mathrm{s}$. band in particular is influenced by profiles 1 and 2. It is obvious from Fig. 5.3la,b that the backward curved profile is the least noisy. It should be, since it approaches a cylindrical profile. That the forward curved blade should be less noisy than the straight one seems at first rather curious. It can be explained in part at least by its slightly smaller depth dimension and the possibility that the air directly in front of the blade may form a comparatively stale higher pressure pocket around which the airstream divides. The apparent 
profile may then be that suggested by the dotted line, profile b, Fig. 5.3la. This profile is less blunt than that of the flat plate.

Two percent B.W. analyses are plotted in Fig. 5.32 for the three profile shapes, station 23 and for profiles 1 and 2, station 5. The results are not indicative of those expected, since the flat plate, not the backward curved blade, generates noise and turbulence of pure toned character. The backward curved blade appears to generate a predominant peak of exactly twice the frequency of that generated by profiles 1 and 2 . Attention is directed again to Gerrard's 33 findings for noise generated from a circular cylinder in an airstream (see spectrum Fig.2.4e p.70) in which he found the frequency of vortex shedding from the cylinder and the acoustic frequency were equal. For a flat plate oriented normal to an airstream the von Kármán frequency formula, $f-\frac{k V}{d}$ c.p.s. for frequency of vortices released from the profile,gives $93 \mathrm{c.p.s}$. for the above situation where:

$k=$ constant $=0.15$ for flat platesl3

$d=$ profile depth projected perpendicular to the airstream $=0.115 \mathrm{ft}$.

$\nabla=$ relative velocity of air and profile $=71 \mathrm{ft} / \mathrm{sec}$ in the duct,$=80 \mathrm{ft} / \mathrm{sec}$ just at the profile.

Using $V=80 \mathrm{ft} / \mathrm{sec}$ results in $f=104 \mathrm{c} . \mathrm{p} . \mathrm{s}$., which coillcides with the peak found in Fig. 5.32. Profile 2 appears to behave equivalently to the flat plate as far as the frequency of vortices produced is concerned, but not for the noise magnitude. Profile 3 is intermediate between a flat plate and a cylinder. 


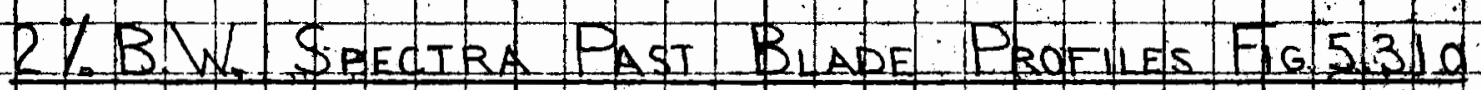
(a) Srafian 5 Fig 5 La

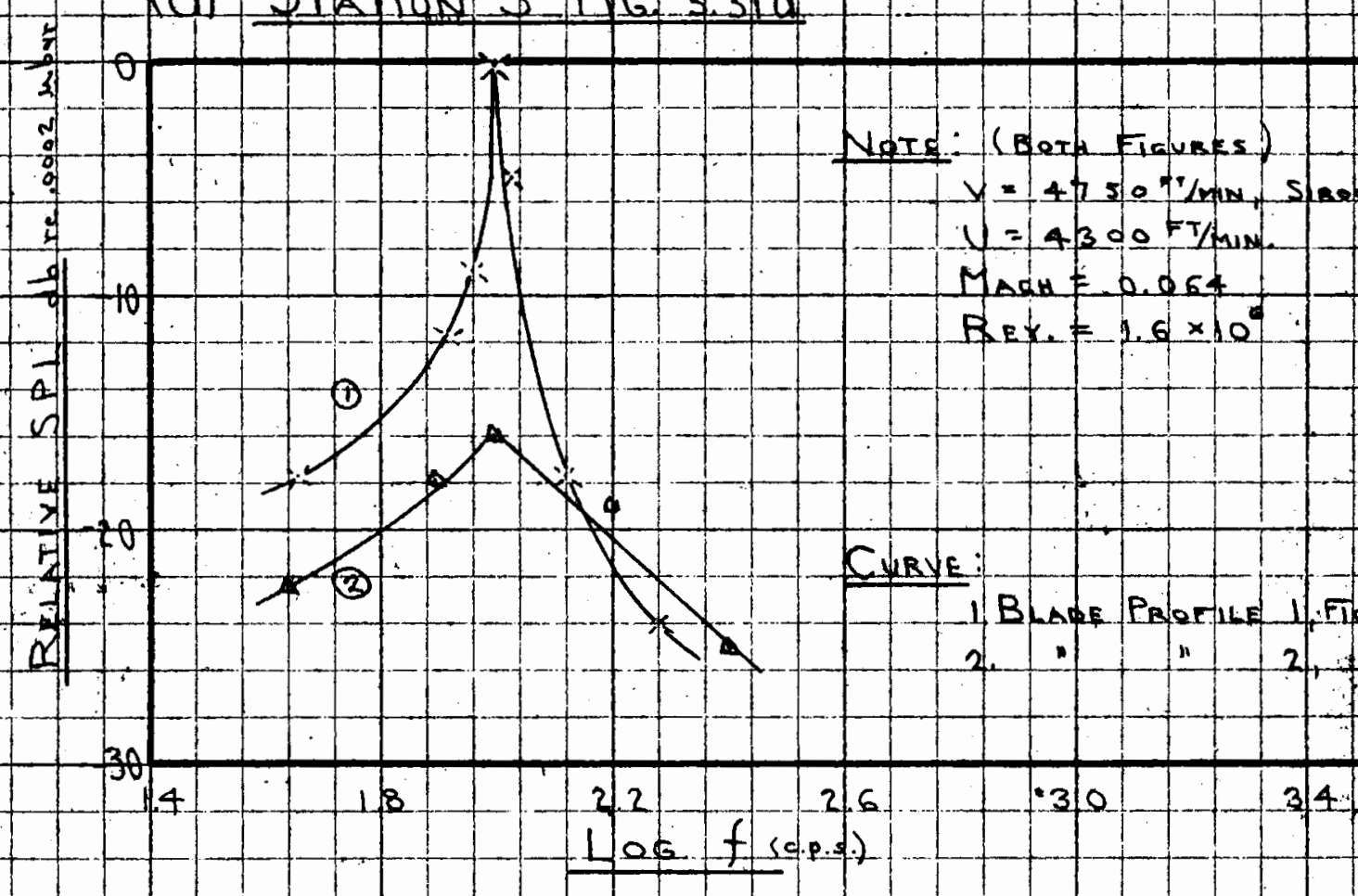

(b) Station 23 Fig. 5.310
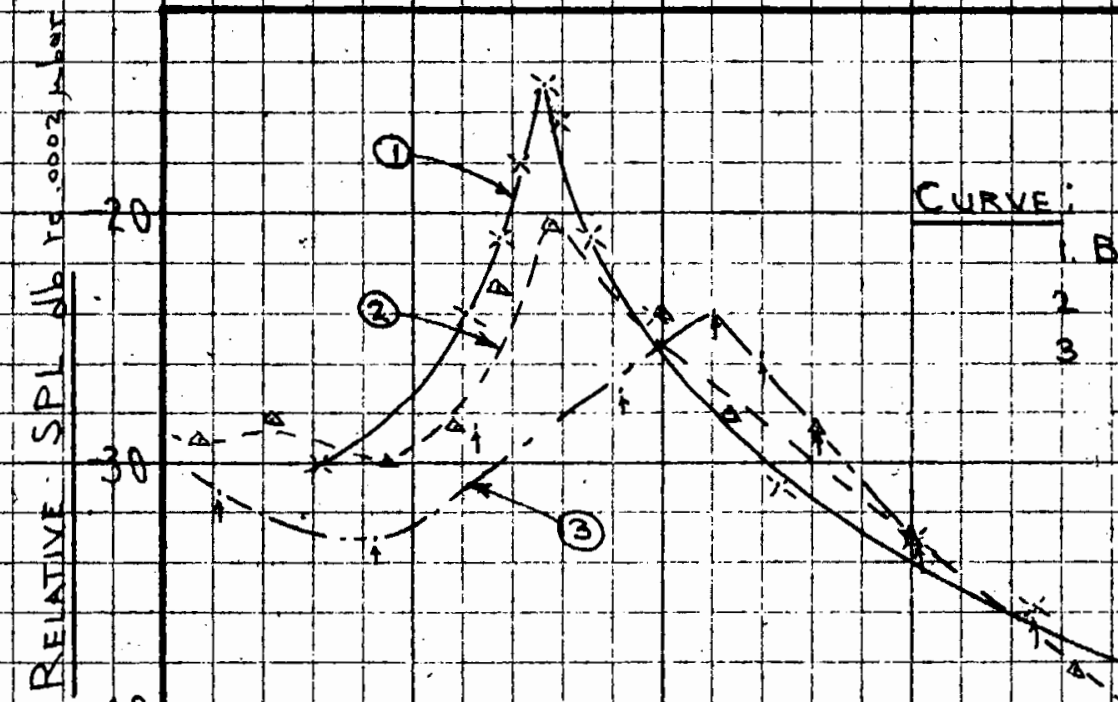

40

1.4

18

2.2

2.6

3.

3. 4

$2=-1-6=$

1.8. $t(+R=)$

$3=213.1$

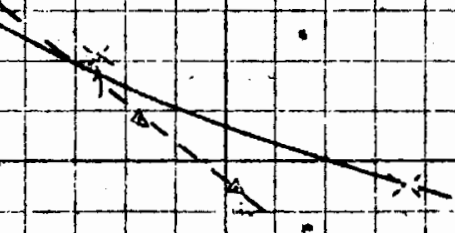

$y=4750 \%$ min sisesed FN MACH $=0.064$

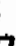


Assigning the constant 0.175 (cylinder is 0.194$)^{13}$ and noting that the projected depth is $0.075^{\prime \prime}$ gives 187 c.p.s. for $f$, which nearly corresponds to the peak for profile 3, Fig. 5.32 . The Rey. number of the flow in the pipe is about $5 \times 10^{5}$. According to Gerrard33 the periodic characteristics of flow past a cylinder in an airstream are replaced by random fluctuations when Rey $>105$. Apparently this does not hold for all flows past bluff obstacles. Gerrard's finding was from studying cylinders rotated by a twirling apparatus, the tested cylinder being parallel to the twirler's axis. Gerrard derived a formula for the intensity of sound from cylinderstwirled in the above manner:

$I=\frac{P_{0} U^{4}}{c} \frac{1^{2}}{r^{2}} f($ Rey. $) \cos ^{2} \theta . . . \quad . \quad(5.3)$

$1=$ length of cylinder.

$r=$ distance of point of measurement from mean cylinder position.

$\theta=$ angle which $r$ makes to the direction of $U$. $f\left(\right.$ Rey) $=\frac{\bar{p}^{2} \bar{r}^{2}}{\left.n p^{2} U^{4}\right|^{2}}$ where $\bar{p}^{2}$ is the mean square sound pressure measured and $n=$ the number of cylinders twirled at one time.

Other symbols per Glossary of Symbols.

Using (5.3), Fig. 6 ref. 33 corrected for $n=1$ to calculate $f$ (Rey), $r=$ unity (duct measurement), and hydraulic mean depth = profile depth, for Rey. number; the intensity of sound at station 23 in the above situation for profile 3 was computed as 0.6 watts $/ \mathrm{m}^{2}$. For the duct of Fig. 1.3 p. 18 this works out to a PWL of $118 \mathrm{db}$ re 10-13 watts. Actual overall measured PWL at this point was $116 \mathrm{db}$, of which $115 \mathrm{db}$ was fan noise. Judging by the accuracy of the measurements, the result is in- 
conclusive. Only the flat plate gives a substantial increase in apparent noise at station 23 (see Fig.5.31a), and this is at a level of $119 \mathrm{db}$ indicating the power level due to the plate alone to be about $117 \mathrm{db}$. The peak of Prir signal, curve 3 Fig. 5.31 a for profile 3 gives an apparent intensity of 0.5 watts $/ \mathrm{m}^{2}$ which is the same order of magnitude as computed for a cylinder of diameter equal to the profile depth of profile 3 .

Fig. 5.33 is of especial interest in view of prior discussions. Fig.5.33a is a $2 \%$ B. H. spectrum for the sirocco fan runner reversed, $b$ for the HS runner in reverse rotation, in the anechoic room. The sirocco runner now simulates a cascade of quarter cylinders moving through air, hence the relatively pure toned spectrum. The HS runner blades, with the tips leaaing, now behave as bluff obstacles, netting a typically flat spectrum. Compare the spectra and overall SPL of Fig. 5.33a, curve 4 Fig. 5.16a; Fig. 5.33b, curve 5 Fig. 5.16b.

\subsubsection{EFFICIENCY OF NOISE GENERATION}

It was mentioned in Art. 5.1.5 that the efficiency of noise generation $\left(\eta_{A}\right)$ expressed as the ratio of noise power from fan to power input to fan was about $10^{-5}$ for fan operation near $\eta_{s} \max$. at the higher speeds. For a wide span of operating conditions $\eta_{A}$ is shown on Fig. 5.34, the upper graph being for the sirocco fan, the bottom for the HS fan. There does not appear to be any similarity between the relation for the Sirocco and HS fan variations except that they are near $\eta_{A}$ of 10-5 at the higher speeds at $\eta_{s}$ max. The HS fan $\eta_{A}$ remains relatively constant for all operating conditions, while the Si- 
Fig: 5.33

page 239

FAN RuNHER ROTATCON REYERSED

2\% B. WL SPECTRA, ANIECHOIC BOOM

(a) Subace RuNWER

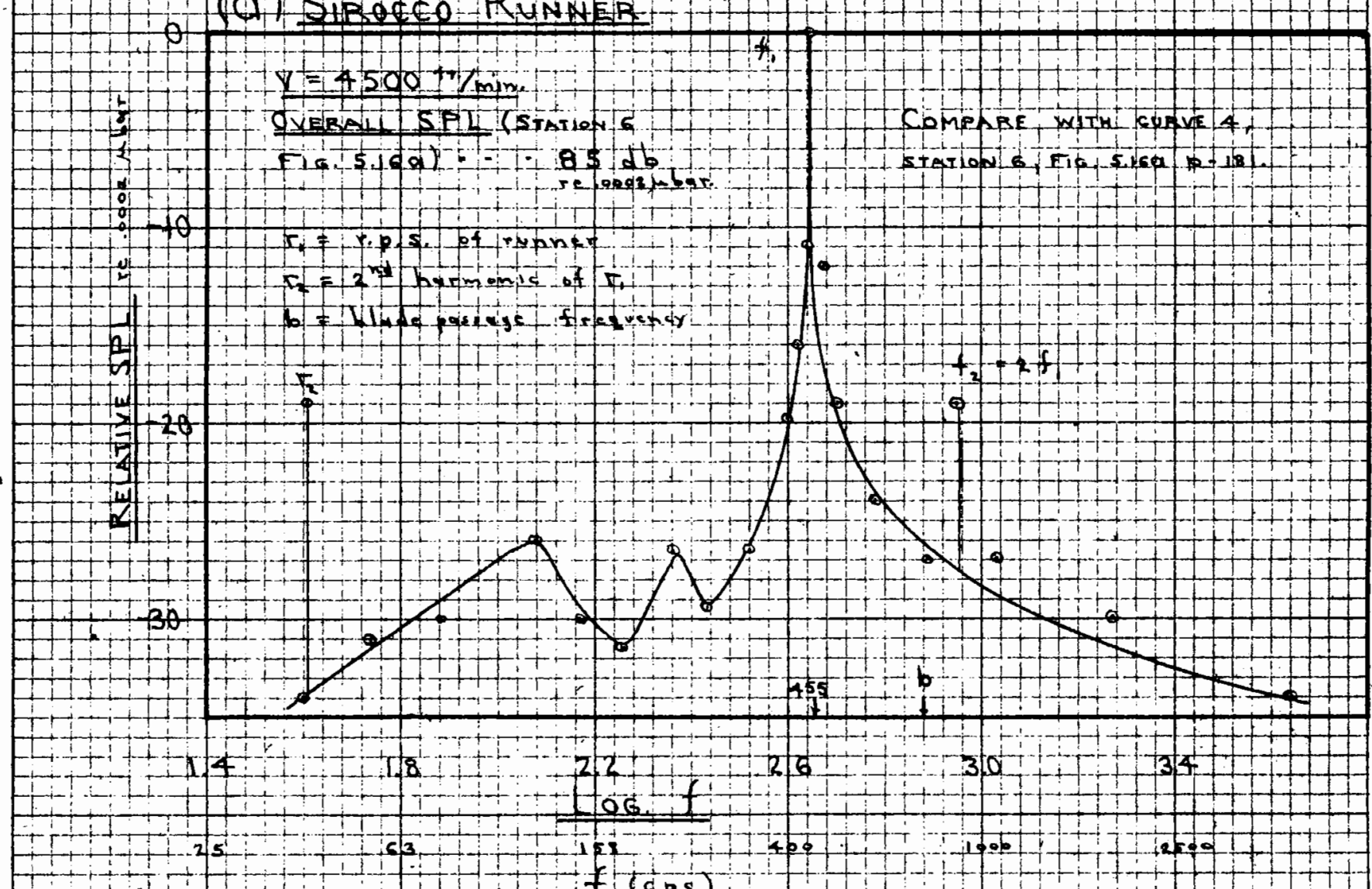

(b) IS S RUNAER

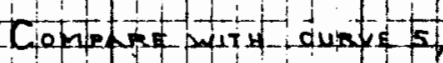

ninan 6 Fia $\operatorname{sichba-162}$

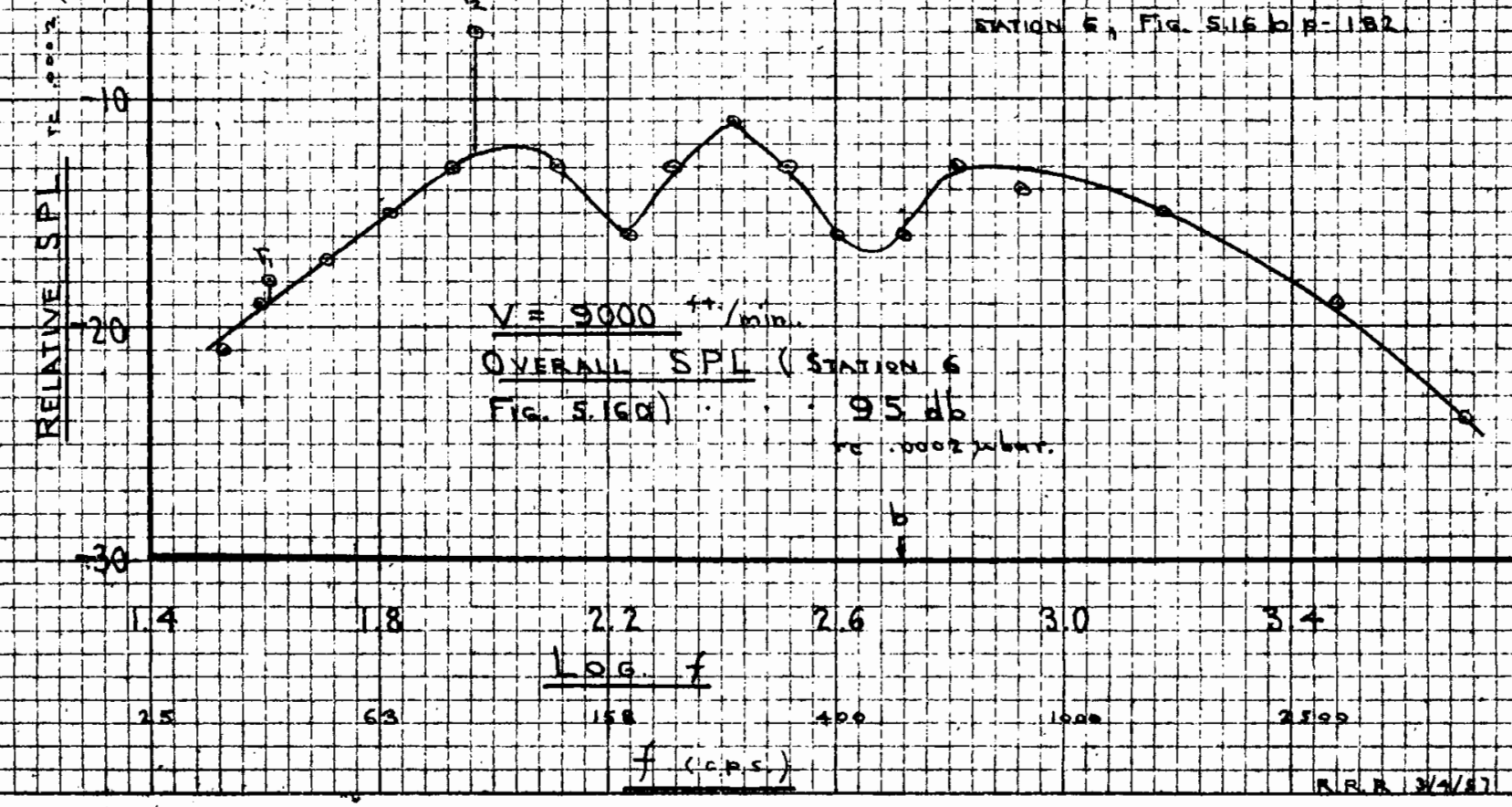




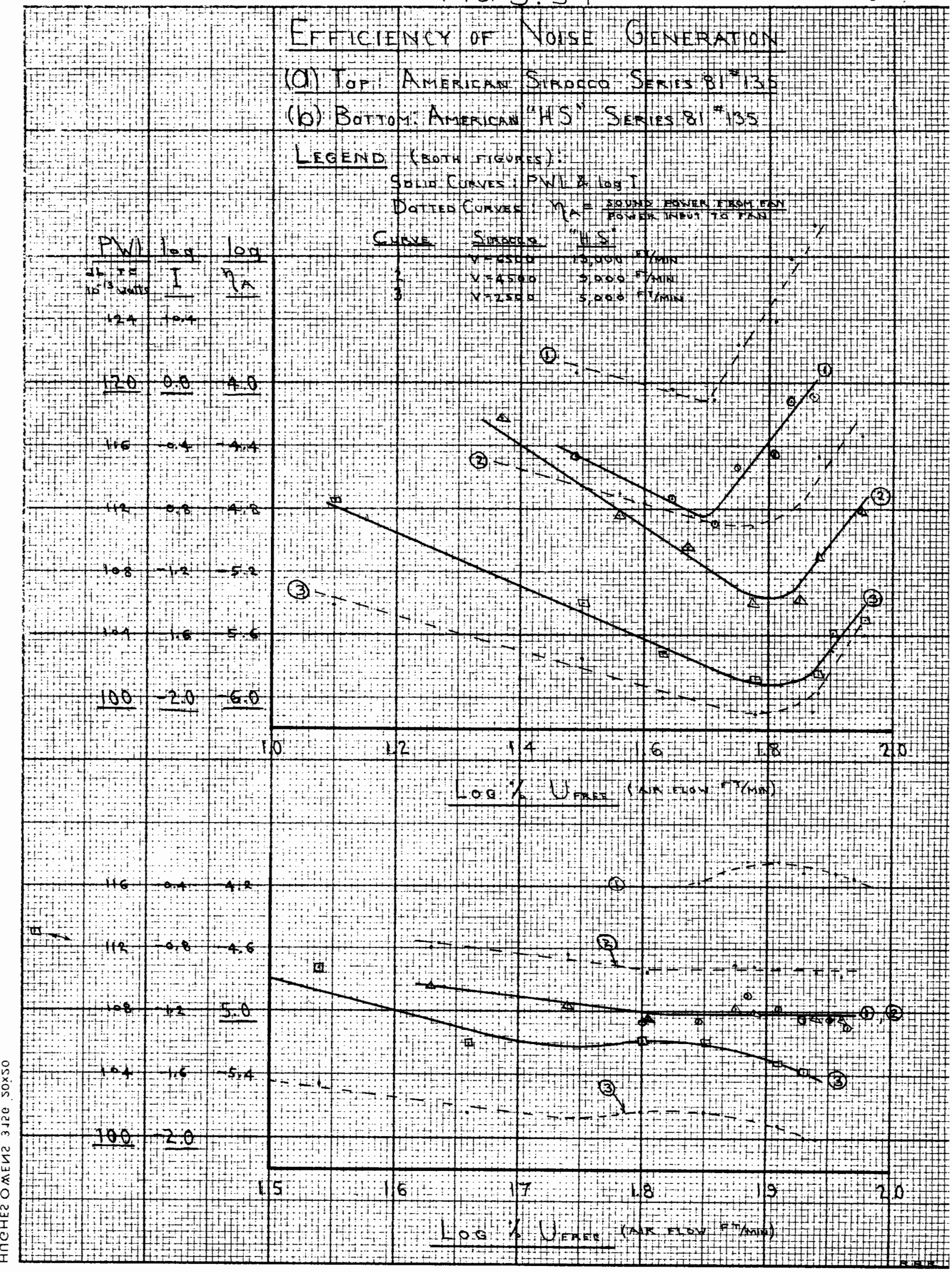


rocco fan $\eta_{A}$ displays a $V$ - shaped variation with $\% U_{F}$; just as PWL does; and increases with speed for a given $\% U_{F}$. An increase in $\eta_{A}$ with $\% U_{F}$ and speed is what would be expected from theory (see Art. 5.5.1).

\subsubsection{SMALI OBSTRUCTIONS VS. NOISE}

Aside from the blade study of Art. 5.3.4, it was desired to determine the effect of the following on fan outlet noise:

1. The "egg-crate" straightener Fig.1.3 and Art.3.1

2. Square mesh of $0.025^{\prime \prime}$ wire spaced on $\frac{1}{2}$ " centers.

3. 0.030 " brass rod

4. Sphere representing the $23 / 4^{n}$ triple spherical windscreen used.

Items 2 and 3 were inserted in place of the egg crate straightener and the two duct sections brought together. The fan was operated over a range of speeds against $F_{\text {syst }}$ low (max. air flow). The windscreen covered microphone was retained at the station shown in Fig. 1.3 p.18. All results proved negative.

The following items were placed at the fan inlet flange and noise measured with the windscreen covered microphone just inside this flange on the runner axis (same station as for inlet PWL measurements Art. 5.1.):

1. 0.030" brass rod across the inlet diameter.

2. $1 \frac{1}{2} " \times 2 \frac{1}{2} "$ block at flange periphery.

3. 2" wide aluminum strip across inlet diameter.

4. Square mesh of $0.17^{\prime \prime}$ rods spaced on 1 " centers across inlet. 
5. Square mesh of $0.025^{\prime \prime}$ wire spaced on $\frac{1}{2}$ " centers.

6. Mesh of $0.015^{\prime \prime}$ wires spaced $0.05^{\prime \prime}$.

If the inlet flow had a marked rotational component, some of these items may give positive results. Except for very minor octave band spectral differences, all results were negative. Items 3,4 and 6 caused measurable decrease in air flow due to increasing inlet resistance. The air flow into the fan is essentially non rotational as fas as fan noise is concerned. (This is a factor in axial fans).

\subsection{ESTIMATED ACCURACY OF RESULTS}

These are based on data presented in Art. 3.2.2 and are the maximum possible (not probable) errors. The effects of standing waves are included unless otherwise mentioned. Considering the average spectra of outlet noise of the fans, the SLM tolerance is taken as \pm 3 db for PWL purposes. The average probable error is based on the deviations of plotted points from the mean in the basic data.

\subsubsection{OVERALL FAN OUTLET NOISE PWL}

Calculation of the maximum possible error proceeds as follows for the example of a $3000 \mathrm{ft} / \mathrm{sec}$ air flow at 2.0 " head water guage.

Independent errors:

SIM $\pm 3 \mathrm{db}$ plus $\pm 1.5 \mathrm{db}$ observational

Standing waves $0,-2 \mathrm{db}$ (maximum overall effect)

Velocity pressure hv, $0.008 \mathrm{n} \mathrm{H}_{2} \mathrm{O}$; Static press.hs, 0.025

Fan speed \pm 1 r.p.m. $\pm 1.5 \%$ 
Fan speed is necessary because flow charts were first drawn, and the proper operating condition determined by fan speed and Fsyst. (the setting of the back panels was quite exact and is neglected here for all practical purposes).

The maximum error at this operating point becomes:

$$
\begin{aligned}
& -(3+1.5)=50 \log \frac{.571}{.555} \times \frac{1.015}{.985} \pm 25 \log \frac{2.025}{1.975} \\
& \text { hr } \% \text { h hs } \\
& \begin{aligned}
&=+(4.5) \\
&-(6.5)
\end{aligned} \pm 1.3 \pm 0.3= \pm 6.0 \mathrm{db} \text { re } 10^{-13} \text { watts. }
\end{aligned}
$$

This result is based on an average increase of intensity with the $5^{\text {th }}$ power of speed and flow and the 2.5 power of static pressure. This of course varies with operating condition and fan type.

A comprehensive listing or plotting of error vs. operating condition and fan type would require pages. A general idea of maximum errors is given in the table below, simply assuming Fsyst is constant and considering only one independent variable.

\section{TABLE 5.3}

SIMPLIFIED TABLE FOR MAX. POSSIBLE ERROR IN PWL ( $\mathrm{db}$ re 10-13 watts)

INDEPENDENT

VARIABLE

Air Flow

(U $\mathrm{ft} / \mathrm{min}$ )

Static Pressure

(hs $\mathrm{H}_{2} \mathrm{O}$ )

Fan Speed*

$(\mathrm{V}=\mathrm{ft} / \mathrm{min})$
MAX. POSSIBLE ERROR

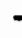

AVERAGE PROBABLE ERROR

PLUS MINUS

\section{0}

3000

5000

PLUS MINUS

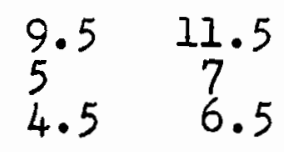

15

34.5

7

6.5

6.5

2500

4500

6500
4.5

$$
\begin{aligned}
& 7 \\
& 7 \\
& 7
\end{aligned}
$$

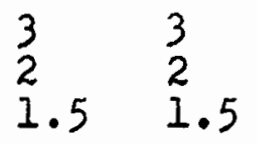

33

$\begin{array}{ll}2 & 2 \\ 2 & 2\end{array}$

$\begin{array}{ll}2 & 2 \\ 2 & 2 \\ 2 & 2\end{array}$


Input Fan

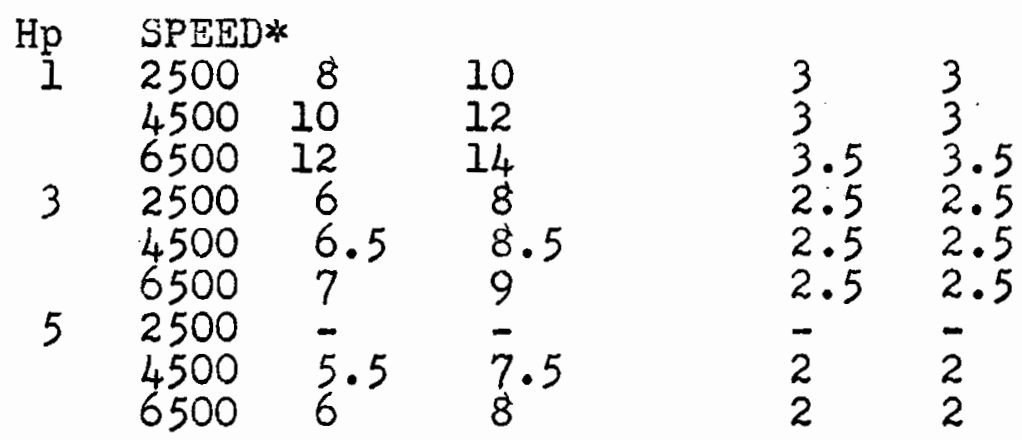

* Speeds given are for the sirocco fan. Double them for the HS fan.

\section{$\underline{5.4 .2 \quad \text { SPECTRA }}$}

OCTAVE BAND

Additional bandwise errors over those of PWL Art.5.4.1 accrue from causes such as standing waves below horn cut-off contained in one band only, cross sectional variations of PWL due to higher order modes, and the addition of an instrument having its own tolerances in series with the SIM. The additional maximum possible errors per octave band are as follows:

MAX. POSSIBLE AVERAGE PROBABLE

$$
\begin{aligned}
& 20-75 c \cdot p \cdot s . \\
& 75=150 \\
& 150-300 \\
& 300=600 \\
& 600=1200 \\
& 1200=2400 \\
& 2400=4800 \\
& 4800-10,000
\end{aligned}
$$

$\begin{array}{ll}3 & -3 \\ 2 & -2 \\ 2 & -2 \\ 2 & -2 \\ 2 & -2 \\ 3 & -3 \\ 4 & -4 \\ 5 & -5\end{array}$

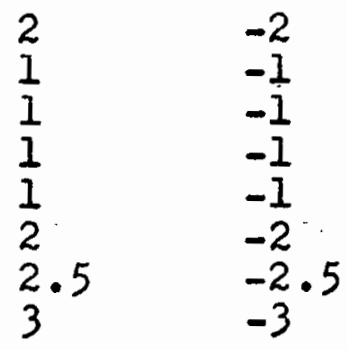

$\mathrm{db}$ re $.0002 \mu \mathrm{bar}$

2\% B.W. SOUND ANALYZER

The independent error of this instrument was discussed in Art. 3.2.2. It is difficult to give errors here for discrete frequencies because of the unknowns of standing waves 
and higher modes at specific discrete frequencies. Errors will be given here as additional maximum possible errors for specific bands over and above the PIL errors Art. 5.4.1. Specific frequencies may be in error more than this, although the method of obtaining and plotting spectra using this instrument averages these out as discussed in Art. 4.

MAX. POSSIBLE AVERAGE PROBABLE

$$
\begin{aligned}
& 25-75 \text { c.p.s. } \\
& 75-1200 \\
& 1200-2500 \\
& 2500-7500
\end{aligned}
$$$$
6-6
$$$$
2
$$$$
-2
$$$$
\text { 4. }-4
$$$$
1 \quad-1
$$$$
8 \quad-8
$$$$
9 \quad-8
$$

3

$-3$

$\mathrm{db}$ re $.0002 \mu$ bar 
6 SUMMARY OF THE MOST IMPORTANT RESULTS; CONCLUSIONS

The solution of ventilating and air conditioning noise demands adequate knowledge of the source. The minimum acceptable knowledge of a noise source is its PWL, spectrum and directivity. Additional desirable knowledge is the origin of this noise and how it can be minimized. Fans cover perhaps the widest range of sizes and types of any machine. Attempts at a universal means of predicting fan noise have to date failed, perhaps for this very reason.

Copious and carefully organized results from the detailed analysis of a forward curved and backward curved centrifugal fan in this research has led to a promising means of universalizing fan noise prediction, assuming the fan is stmucturally sound and well installed. The method is this: Each homologous series of fans can have its outlet noise power defined as one $\mathrm{V}$ - curve of specific noise power (PWL) $\mathrm{s}$. log specific speed (Ns). At present this curve cannot be well predicted, but must be obtained from careful measurement on a typical fan or fans of the series. The (PWL) minimum corresponds closely to maximum static efficiency, and almost exactly with maximum total efficiency. A set of laws involving PWL or $I$ as a function of $U$, hs, $n, H p$ and syst. (see glossary of symbols) can be derived from:

$$
\text { PWL }=(P W L)_{s}+10 \log \left(Q h s^{2}\right) \text { db re 10-13 watts (5.1) }
$$

These laws will differ for each fan series, because each slope of the (PWL) - log Ns curve implies a different set of laws. This variation is not to be compared with some of the verified laws already established for certain cases of fan operation, as 
these latter apply only at a single point of rating on the $\eta_{s}$ - Ns curve, while the proposal of this thesis is for operation at any point on the curve.

Since the (PWL)s - $\log N s$ curve is generally $V$-shaped, one set of laws applies at and above the $\eta_{s}$ max. point whereas the other applies below $\eta_{s} \max$. Thus to completely describe the noise changes with the point of operation of a fan series requires two distinct law sets. The variations at $\eta_{s}$ max. are most accurately described by those laws applicable at specific speeds above that at $\eta_{s} \max$.

It is possible there is some relation between the $\eta_{s}-$ $\log N$ s curve and the (PWL)s - log Ns curve. It is not in the slope of the $\eta_{s}-\log \mathrm{Ns}$ curve, but experimental evidence suggests a possible relation between the slope of the (PWL)s $\log \mathrm{Ns}$ curve and the breadth and magnitude of the $\eta_{s}$-maximum, or blade profile, as illustrated in Fig. 6.1a. Further research is indicated to establish this.

The $(P W L)_{s}$ - log. Ns curves contained in this thesis are for fan output noise with either no duct on the outlet, or a duct of the same cross sectional area. Air flow velocity is the increasingly governing noise factor above $\eta_{s} \max$. (specific speeds above that at $\eta, \max$.$) . The position of the right hand$ branch of the $(P W L)_{s}$ - log Ns curve depends to an extent on $U$, hence on the area of the attached duct. If the fan and a length of outlet duct are taken as the noise source unit, the right hand branch of the (PWL) $-\log N s$ curve can be expected to change in a manner suggested in Fig. 6.Ia, as duct area varies with respect to the fan outlet area. Additional research 


\section{F16.6.1}

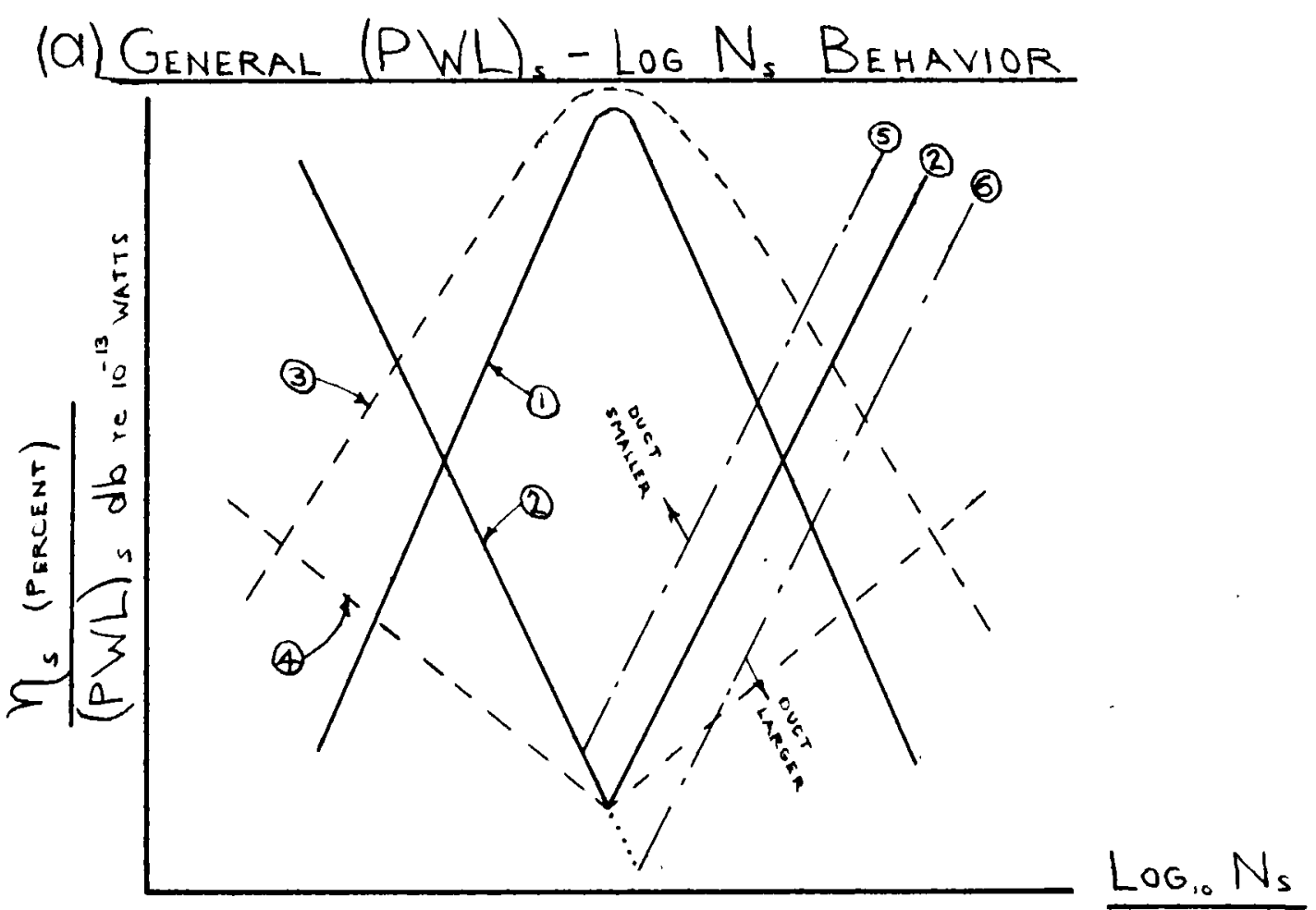

Curve: (1) Forward curved blade centrifugal fan etficiency curve.

(2) (PWL) S CURVE FOR FAN IN Q .

(3), (1) - (1) (2) DITTO EXCEPT FOR BACKWARd cuRVEd BLADE FAN.

THE LOG N, ABSCISSAL SCALE IS HIGHER THAN FOR D, 2 - CURVES ARE SUPERTOSED FOR CONVENIENCE.

(S) CURVE (3) SHITTS WHEN FAN CONNECTED TO A DUCT OF AREA SMALLER THAN THE FAN OUTLET AREA IS TAKEN AS THE NOISE SOURCE UNIT. THE same applies to curve Q.

Q-Q DitTo, EXcErt DUCT AREA LARGER THAN FAN OUTLET AREA.

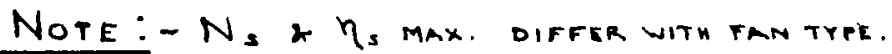

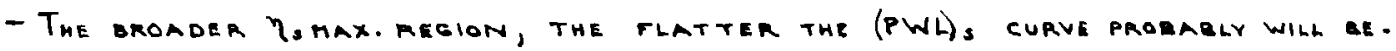

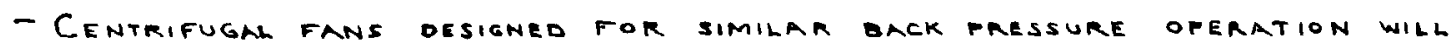
HAVE NEARLY THE SAME (PWL) S MIN. REGARDLESS OF AIR VOLUME OR BLADE PROFILE. NOISE SPECTRA HOWEVER WILL DIFFER.

(b) Generalized Centrifugal Fan Noise Spectrum

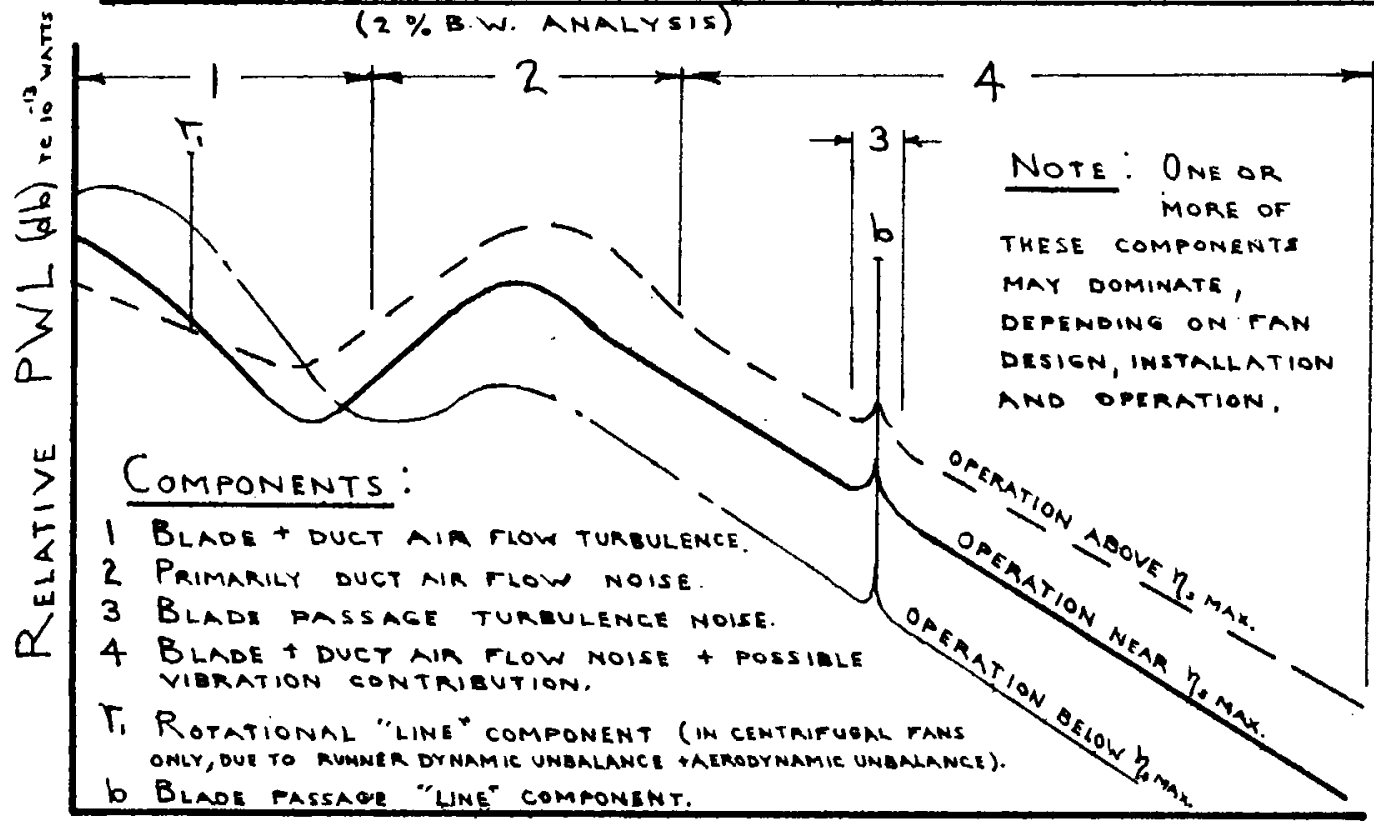


is required to establish this law.

Why is the left hand branch, which is governed mainly by back pressure effects, not altered? It is suspected, as Goldman and Maling 4 did, that the minimum noise from a fan attached to a system is dependent on the operating pressure (pressure differential between inlet and outlet). This does not mean that pressure itself or the slight increase in air density can generate noise. It does mean that the fan design details and operation details hinge on back pressure. Once this is established, the flow can be varied to suit the need by fan size. For a given air flow, high pressure operation will generate more noise than low pressure operation because:

1. Each blade must convey more power to the airstream.

2. High pressure blades are deeper. The consequent air acceleration and turbulence about them will be larger, than for a shallow blade. (eg. The difference between noise from a small and large bluff obstacle in a turbulent airstream).

3. For a given air volume, higher pressure operation generally means higher tip speed.

4. Blade profile varies with the pressure it is required to develop. This in itself will not change the noise but will alter the spectrum over that of a volume fan, usualiy shifting the noise power distribution to somewhat higher frequencies. Loudness level is therefore increased.

5. For a given air flow velocity, it is quite possible the nature of turbulence in the flow differs with pressure in such a manner as to increase the output noise. This will 
be one object of an extension of this research, as mentioned further in Art. 7.

Since the (PWL) $)_{5}^{-} \log$ Ns curve must be obtained experimentally for each fan series, until further research evolves a means of predicting this reliably, it is little additional work to take a typical $2 \%$ B.W. spectrum at the same time. With a (PWL) s - $\log \mathrm{Ns}$ curve and a typical spectrum (say one at $\eta_{s}$ max., one above and one below), the acoustician or customer is greatly helped in predicting the ventilating noise in a given room, or to design attenuating devices for the system. For ducted fans, these two pieces of information are a sufficient minimum to describe the source.

Fan noise spectra reveal noise genesis. A typical spectrum for centrifugal fans of the volume type under three variant load conditions are sketched in Fig. 6.1b. The spectrum will change little with speed for an equivalent operating condition, the main alteration being a shift in some components. (This does not hold of course at low speeds where negligible pressure is produced across the runner blades). The noise components comprising the spectrum of Fig. 6.1b are universally present in fan noise and differ only in their relative magnitude and frequency position. A universal fan noise spectrum may be thought to exist. containing the following major components to greater or lesser degree. They are in their approximate order of importance for the two fan types discussed in this thesis:

1. Blade turbulence spectrum. This will vary from a large line component with many harmonics for the propellor, to 
a wideband almost flat spectrum for the many bladed sirocco fan.

2. A wideband component with broad maximum centered at the lower frequencies (100 to 300 c.p.s.) representing noise from boundary turbulence in the air flow. This will grow in magnitude and width, and will shift upwards in frequency as flow velocity increases. The turbulence causing this noise component tends towards isotropy. This component will not generally be significant for flow velocities < Mach number of 0.05 . Most ventilating systems are designed with flow velocities less than this except in the main duct where they are approaching Mach $=0.1$ in advanced high speed systems52. This component of noise, once generated in a high flow velocity section, can propagate through a lower velocity section, hence its importance in considering fan noise. If the duct area $>$ fan outlet area, this noise can be generated at the fan outlet (with modifications in the turbulent structure of the flow compared to long duct flow). Design fan outlet velocities approach Mach 0.1 as a maximum today for ventilating purposes.

3. Low frequency noise centered below, near, or above the lower audio limit, depending on fan type and flow conditions. This random continuous spectrum component is caused by some contribution from 2, plus additional noise resulting from a scale of eddying and/or large scale turbulence not of isotropic nature due to the design of the fan, changes in system area, discontinuities or obstructions in the system. This component is difficult to as- 
sess with current microphone windscreen designs. Eddying in the flow for example may cause a low frequency noise output due to reaction between it and a duct wall, but this noise may be masked by the eddying producing pressure fluctuations at the microphone which are not representative of the actual low frequency noise pressure fluctuations. The only solution may be to locate the microphone away from the flow, not within a windscreen in the flow. The type of windscreen used was shown to provide otherwise good protection from non acoustic pressure fluctuations at the higher frequencies (above about 100 to 150 c.p.s.) for noise measurements in air flow in a duct.

4. High frequency spectrum depends on the fan design, speed, vibration and flow. If air flow and blade turbulence are the major contributors, the high frequency end of the spectrum can be expected to decline at a rate of about $5 \mathrm{db}$ per octave from the last salient.component, which may be due to 1,2 or vibration.

5. Dynamic runner unbalance will cause generation of a rotational frequency (r.p.m./60 c.p.s.) line component and one or more of its harmonics. These may, or may not, add appreciably to the overall noise. They will be significant in higher speed centrifugal fans, but will not be too important in most low speed centrifugal fans and axial, propellor and tubeaxial fans of any design.

6. Vibration may add an unpredictable measure of noise to the spectrum at certain frequencies, or over narrow bands of frequencies, but generally it will not contribute 
significantly to the outlet noise spectra of well designed and installed fans.

Until more is known from further fan tests, fan noise spectra should be obtained experimentally along with the (PWL)log Ns curve for supplying fan users with fan noise information. The Sirocco fan can, to a very rough approximation, be represented by an octave band spectrum declining from the first band at the constant rate of $5 \mathrm{db}$ per octave. This agrees with Beranek, Kamperman and Allan3. This does not in the least suggest a uniform detailed spectrum. For operation at and above $\eta$. max. (eg. specific speeds above those for $\eta_{s}$ max.), the octave band spectrum is closer to a $4 \mathrm{db} /$ octave decline, whereas for operation at specific speeds below $\eta_{s} \max$. the spectrum should be represented by a 6 or 7 db/octave decline. This is what Goldman and Maling 4 also found.

\section{CONCLUSIONS}

The above constitutes the major findings regarding centrifugal fan noise PWL, spectra and origin. Other findings of importance are:

1. Centrifugal fans of forward and backward curved blade design have an efficiency of noise generation of 10-5 at and near $\eta_{s}$ max. This is a guide in assessing the probable overall PWL of these fans, and also in establishing the trough value of the (PWL) - $\log N s$ curve. Estimated PWL near $\eta_{s} \max$.

$=10 \log \frac{W_{\text {tan }}}{W_{\text {ret }}} \mathrm{db}$ re $10-13$ watts where Wfan $=10-5 \times$ Power input to fan in watts. 
For the backward curved blade $f a n$, this is manifested over a wide range of the $\eta_{s}-N s$ curve.

2. Commonly published fan noise laws are not applicable unless restricted to one point of rating on the $\eta_{s}-\mathrm{Ns}$ curve; and even then they do not always apply for fan operation at specific speeds below $\eta_{s} \max$.

3. Fan noise intensity increase with speed (Fsyst constant) can obey laws varying from at least 4 to 7, depending on fan design. The backward curved runner will be close to 4 , the forward curved runner near 6 or 7 . The radial runner and axial fans are predicted to obey the $5^{\text {th }}$ to 6 th power speed law. There is evidence for these conclusions in Art. 2.2 in addition to the experimental results of this thesis.

4. At $\eta_{s} \max .$, the forward and beckward curved blade centrifugal fans produce equal noise. The forward curvedblade generates the most pleasing spectrum from the annoyance viewpoint. At operation away from $\eta_{s} \max .$, the forward curved blade runner generates more overall noise power than the backward curved blade runner, but there is some compensation in the more pleasing spectrum.

5. Back pressure (or more accurately point of operation with respect to $\left.\eta_{s} \max .\right)$ has significant effect on noise level and spectrum. The more forward curved the blade profile, the more pronounced this effect will be. The more backward curved, the less the effect. The higher the back pressure, the steeper the octave band spectrum.

6. Broadly generalizing from the results; the quietest sounding centrifugal fan is one having the optimum com- 
bination of the largest number of shallow forward curved blades and lowest speed as is aerodynamically and practically feasible. The backward curved blade emits less noise, but unfortunately delivers less air unless operated at a higher tip speed than the forward curved blade. For a given tip speed the forward curved blade runner rotated alone in anechoic space generates a power level some $15 \mathrm{db}$ higher than the backward curved blade runner. For equivalent aerodynamic operation however, the difference is only $2 \mathrm{db}$, but the forward curved blade runner possesses the most pleasing spectrum and ultimately results in the lowest loudness level. Perhaps a composite curvature blade design (reverse curve) will prove the least noisy. Over and above this, plus operation of the fan at maximum efficiency, the lowest practical speed and outlet flow velocity, there is little that can be done to minimize generated fan noise.

7. The rotational line component and harmonics can be minimized in centrifugal fans by accurately balancing the runners dynamically and providing rigid construction. Although the construction was good, dynamic runner balance of the two fans tested could be improved.

8. Installation for minimum noise requires:

(a) The fan be located either remote from areas to be kept quiet, or in a sound proof room.

(b) A vibration break between fan and duct.

(c) A rigid mounting on concrete or the like. If there is an element of dynamic runner unbalance, as there always is, installation on vibration isolators may 
greatly increase the rotational component and its harmonics propagated down the duct.

9. Any aerodynamic dissymetry will increase the rotational component and its harmonics. Such dissymetry may be caused by an eccentric runner (even if it is possible to perfectly balance it), unequal blade spacing or unequal blade profile. This may become a maintenance problem if unfiltered inlet air causes deposits of grime on blades.

10. Obstructions such as screens, small blocks and rods at the inlet, or screens and slender rods or obstruction small compared to the outlet area at the outlet, have no effect on the overall fan noise PWL and only minor spectral effect.

11. A fair indication of overall Pin at the outlet of a centrifugal fan is given by placing the windscreen covered microphone on the axis of the inlet eye, just within the flange end. The result will be on the low side and may not be as close for operation at specific speeds below $\eta_{\text {s }}$ $\max$. as above. Adding $3 \mathrm{db}$ to the SPL measured near $\eta_{\mathrm{s}}$ max. should net the approximate outlet PWL, assuming the fan inlet and outlet areas are nearly equal. The inlet spectrum however is quite unlike that at the outlet.

12. Averaging of fan noise over the duct section is necessary above the cut-off frequency for the $(0,1)$ mode. The microphone should be located in such a position as to approximately sample the average of the standing waves through the frequency range below horn cut-off. This requires preliminary calculation and longitudinal standing wave measurements, plus some knowledge of the expected 
fan spectrum below horn cut-off.

13. The triple spherical windscreen used in this research is unreliable in anisotropic flow at frequencies below 150 c.p.s. The larger the windscreen and the more spaced layers of high air impedance, zero acoustic impedance material used, the better, up to the point where the projected windscreen area becomes an appreciable fraction of the bounded airstream cross section within which measurements are taken. The windscreen used herein constituted the best available compromise to date, but further research is indicated. In the duct section where most of the measurements were taken, the flow was random and approached isotropy. (according to experiments involving a mesh in an airstream, Art. 1.2).

14. If a ventilating duct is well braced, stiff, or damped, no significant noise will accrue therefrom. Noise propagating within will be attenuated 15 to $30 \mathrm{db}$ overall depending on material and construction, for conventional unlined ducts.

15. Ventilating duct transitions having tapers in excess of about $10^{\circ}$ will not only be aerodynamically poor, but may result in added noise generation due to the new scale of turbulence established.

16. The matched acoustic waveguide technique of measuring fan noise is a generally expedient, practical and accurate method. A smaller acoustic waveguide system than that of Fig. 1.3 p.18 is not recommended for testing of fans having in excess of one foot runner diameter. The larger the horn and termination, the better. Due to the nature of 
pressure - velocity component relations of air at the blades of a centrifugal fan, flow from such fans in a duct is not generally symmetrical. Those engaged in further work using this equipment should be satisfied with nothing less than the 20 flow measuring stations recommended in Ref.54. The flow distribution changes with load and fan speed. Manufacturers' data should be used only as a check, not the basis for flow data in fan noise research.

Of the various major parameters upon which fan noise depends (at low Mach numbers for clean air):

$$
\begin{aligned}
& \text { PWL }=f \text { (U, hs, Hp, } n, N, I, D, d, \text { Syst., P, A) } \\
& U=\text { Flow velocity } \\
& \text { hs }=\text { Back pressure } \\
& \text { Hp }=\text { Horse power to fan } \\
& n \quad=\text { Fan rotor speed } \\
& N \quad=\text { Number of rotor blades } \\
& I \quad=\text { Blade length (rotor width) } \\
& D \quad=\text { Rotor diameter to the blade tips } \\
& \text { d }=\text { Blade depth } \\
& \text { Syst }=\text { System attached to fan } \\
& \text { P }=\text { Blade profile } \\
& \text { A }=\text { Ducted fan inlet and outlet area, }
\end{aligned}
$$

$N, I, D, d, P$ are the responsibility of the fan manufacturer, and the remainder are under control of the ventilating engineer. The acoustician is concerned with them all. If noise cannot be minimized to the satisfaction of the customer by proper fan design, selection, installation and operation, only one para- 
meter can be altered; the connected system. Since such alteration can prove very expensive and time consuming, everything possible must be done to assure proper selection of the other parameters. It is the responsibility of the acoustician to lend guidance and information to those concerned with any system used to move air in public places, so that a satisfactory installation from the noise viewpoint can be obtained. The work has just begun. This research pours additional information into the growing stream of noise studies. Much more research is required, for we are just now becoming acoustically acquainted with the source characteristics, after which noise reducing fan designs must be studied, and finally research on the entire ventilating and air conditioning system intensified to try and separate "the inseparable" from moving air: noise. 


\section{RECOMMENDATIONS}

1. Conduct further research in support of the contents of this thesis, and implications and predictions made therein. In particular to:

(a) Determine a means of estimating the (PWL) - log Ns curve given a fan type and its $\eta_{s}-\mathrm{Ns}$ curve.

(b) Determine the noise law associated with ducts of varying diameter applied to a given fan outlet, when the fan plus duct is considered as the noise source unit.

(c) Select two fans of the same series as tested herein, but of different size, to verify the results.

2. If I (a) proves negative, I (c) positive, make measurements on all the common fan types (centrifugal and axial) used in North America today to establish a "standard" set of (PWL) - $_{s} \log$ Ns curves and typical spectra, suitable for publication in a fan or ventilating handbook, and for distribution as a bulletin.

3. By extensive flow pictures and measurements, show the effects of relative back pressure and flow (eg. percent free flow of a $f a n)$ on the scale of turbulence at the $f a n$ runner and in the duct. Try to determine whether it is back pressure that governs minimum noise in a ventilating system, and if so, if it can be reduced. This was to have been part of this research program, time permitting. Work has begun in fabricating duct sections with windows and in assembling a smoke generator capable of producing a controlled, dense, white, optically opaque, smoke which can be inserted as ele- 
ments into a pressurized ventilating system or near the fan blades for visual turbulence studies. This work is to be continued.

4. The effect of air turbulence of isotropic and anisotropic nature at different Mach numbers on microphone windscreens should be investigated with a view to establishing their low frequency efficiency. It must be kept in mind that if the acoustic waveguide noise measuring technique is to continue, the windscreen must be restricted in size, in addition to being efficient over the audio range. Failing this, it may be possible to predict the proportion of low frequency signal generated due to actual noise, rather than turbulence fluctuations reaching the microphone diaphram. If all this gives negative results, the solution may be Goldman and Maling's 4 waveguide with the shunt air exhaust. Trying to design such a shunt, reflecting high acoustic impedance into the waveguide over the entire audio spectrum, and not causing additional turbulence or noise propagation into the waveguide, may prove a formidable task indeed. Until further data is available on windscreen behaviour in a turbulent stream (as opposed to a screen being driven through quiescent air), the flow at a windscreen covered microphone should be kept below Mach 0.05 if possible, with current windscreen designs. This applies only for turbulence approaching isotropy.

5. Research is indicated on fan runner and blade design with a view to minimizing noise without deterrent to the aerodynamic performance of the blade or fan. 
6. Acoustically investigate different aspects of the ventilating system such as bends, dampers, transitions and louvers in air flow. Perform research on impedance mismatching (noise reflecting, air admitting)devices, filters and such, under the influence of air flow.

7. It may prove more convenient and reliable for the fan manufacturers to submit fans to a fan noise research center having the trained personnel and specialized equipment necessary to acoustically analyse and appraise fan noise, rather than trying to incorporate this as part of the standard fan Test Code. A simple noise measurement could be made while aerodynamically testing the fans to determine whether the fan noise falls within limits specified by the acoustician.

8. The 2\% B.W. Sound Analyzer should be the standard instrument (plus graphic or visual recorders) for measuring fan noise spectra. This is necessitated by the composite continuous - line component nature of the spectra. Bandwise spectra can be deceiving when line components exist. 


\section{BIBLIOGRAPHY}

(The first page of each article only is given in brackets)

- 1. L.L. Beranek, J.L. Reynolds \& R.E. Wilson; Apparatus and Procedures for Predicting Ventilating System Noise;

J. Acoustic. Soc. Amer.,Vol.25, (313), March 1953.

2. C.F. Peistrup \& J.E. Wesler; Noise of Ventilating Fans; J. Acoustic. Soc. Amer., Vol 25, (322), March 1953.

3. L.I. Beranek, G.W. Kamperman \& C.H. Allen; Noise of Centrifugal Fans; J. Acoustic. Soc. Amer., Vol 27, (217), March 1955.

4. R.B. Goldman \& G.C. Maling; Noise from Small Centrifugal Fans; Noise Control, Vol 1, (26), November 1955.

5. C.G. Van Niekerk; Measurement of the Noise of Ducted Fans; J. Acoustic. Soc. Amer., Vol 28, (681), July 1956.

6. A. Bredahl; Designing and Testing of Microphone Windscreens; Master of Engineering Thesis, McGill University, 1956.

7. G.E. Chipps; Noise Produced by a Centrifugal Ventilating Fan; Master of Engineering Thesis, McGill University, 1956.

8. Noise - Several Methods to Prevent or Correct Noises Made by Fans; Heating - Piping and Air Conditioning, Vol 1 (3II), August 1929.

9. G.T. Stanton; Noise and Ventilation; Heating - Piping and Air Conditioning; Vol 2, (1049), December 1930.

10. V.0. Knudsen; Recent Development in Sound Control and their Importance in Air Conditioning; Heating - Piping and Air Conditioning; Vol 6, (245), June 1934.

11. L.S. Marks \& J.R. Weske; The Design and Performance of an Axial - Flow Fan; Transactions of the Amer. Soc. of Mech. Engineers, Vol 56, (807), 1934. 
12. K.D. McMahan; The Noise Problem in the Application of Fans; J. Acoustic. Soc. Amer., Vol 7, (204), 1936.

13. E.Z. Stowell \& A.F. Deming; Vortex Noise from Rotating Cylindrical Rods; J. Acoustic. Soc. Amer., Vol 7, (190), January 1936.

14. F.P. Bleier; Design, Performance \& Selection of Centrifugal Fans; Heating \& Ventilating, Vol 45, (68), September 1948.

15. G.K. Batchelor; Kolmogoroff's Theory of Locally Isotropic Turbulence; Proceedings of the Cambridge Philosophical Soc., Vol 43, (533), 1947.

15-1 A.N. Kolmogoroff; Most of his work on the Theory of Locally Isotropic Turbulence appeared in Comptes Rendus (Doklody) de l'Academie des Sciences de I'U.R.S.S. (C.R. Acad. Sci. U.R.S.S.), which published short up-to-date accounts of current work in English, French and German languages.

16. H.L. Dryden; A Review of the Statistical Theory of Turbulence; Quarterly of Applied Mathematics, Vol 1, (7), 1943.

17. H.C. Hardy; Unsolved Basic Physical Research Problems in the Field of Noise; J. Acoustic. Soc. Amer., Vol 24, (767), 1952.

18. M.J. Lighthill; On Sound Generated Aerodynamically, I General Theory; Proc. Royal Soc. London, Vol A 2l1, (565), March 1952.

18'-1 J.H. Gerrard, 1953 PH.D. thesis, Manchester.

19. M.J. Lighthill; On Sound Generated Aerodynamically; II Turbulence as a Source of Sound; Proc. Royal Soc. London, Vol A 222, (1), 1954 .

20. H.C.H. Townend; Statistical Measurements of Turbulence in the Flow of Air Through a Pipe; Proc. Royal. Soc. London, 
Vol. A 145, (180), 1934.

21. L.F.G. Simmons \& C. Salter; Experimental Investigation \& Analysis of the Velocity Variations in Turbulent Flow; Proc. Royal Soc. London; Vol. A 145, (212), 1934.

22. G.I. Taylor, Statistical Theory of Turbulence; Proc. Royal Soc. London; Vol. A 151, (421), 1935.

23. I.F.G. Simmons \& C. Salter; An : Experimental Determination of the Spectrum of Turbulence; Proc. Royal Soc. London, Vol. A 165, (73), 1938.

24. G.I. Taylor; The Spectrum of Turbulence; Proc. Royal Soc. London, Vol. A 164, (476), 1938.

25. W. Heisenberg; On the Theory of Statistical \& Isotropic Turbulence; Proc. Royal Soc. London, Vol. A 195, (402), 1949.

26. E.W. Comings, J.T. Clapp \& J.F. Taylor; Air Turbulence \& Transfer Processes; Industrial \& Engineering Chemistry, Vol. 40, (107), June 1948.

27. S.G. Kovásznay; The Spectrum of Locally Isotropic Turbulence; The Physical Review, Vol. 73 (2nd Series), (1115), 1948.

28. 4.A. Townsend; The Structure of the Turbulent Boundary Layer; Proc. Cambridge Philosophical Soc., Vol. 27, (375), 1951.

29. J. Laufer; The Structure of Turbulence in Fully Developed Pipe Flow; Applied Mechanics Review, Vol. 7, (29), Abstract 208, Jan., 1954.

30. G.K. Batchelor; Pressure Fluctuations in Isotropic TurbuIence; Proc. Cambridge Philosophical Soc., Vol. 47, (359), 1951.

31. J.E. Moyal; The Spectra of Turbulence in a Compressible Fluid, Eddy Turbulence and Random Noise; Proc. Cambridge 
Philosophical Soc., Vol. 28, (329), 1952.

32. I. Proudman; The Generation of Noise by Isotropic Turbulence; Proc. Royal Soc. Iondon, Vol. A 214, (119), 1952.

33. J.H. Gerrard; Measurements of the Sound from Circular Cylinders in an Air Stream; Proc. Physical Soc. London, Vol. $68 \mathrm{~B},(453), 1955$.

34. N. Curle; The Influence of Solid Boundaries on Aerodynamic Sound; Proc. Royal Soc. London, Vol. A 231, (505), 1955.

35. O.M. Phillips; On the Aerodynamic Surface Sound from a Plane Turbulent Boundary Layer; Proc. Royal Soc. London, Vol. A 234, (327), Feb. 1956.

36. O.K. Mawardi \& I. Dyer; On Noise of Aerodynamic Origin; J. Acoustic. Soc. Amer., Vol. 25, (389), 1953.

37. H. Medwin, A.C. Pietrasanta \& I. Dyer; Results of Noise Measurements on Wind Tunnels; J. Acoustic. Soc. Amer., Vol. 27, (1015), (extráct), 1955.

38. O.K. Mawardi; On the Spectrum of Noise from Turbulence; J. Acoustic. Soc. Amer., Vol. 27, (442), May 1955.

39. R.H. Kraichnan; Pressure Field within Homogenous Anisotropic Turbulence; J. Acoustic. Soc. Amer., Vol. 28, (64), Jan. 1956.

40. R. H. Kraichnan; Pressure Fluctuations in Turbulent Flow over a Flat Plate; J. Acoustic. Soc. Amer., Vol. 28, (378), May 1956.

41. D.E. Neston; The Theory of the Propagation of Plane Sound Waves in Tubes; Phys. Soc. Proc. (London), Vol. 66 B, (695), 1953.

42. R.F. Lambert; Acoustical Propagation of Higher Order Modes; J. Acoustic. Soc. Amer., Vol. 27, (790), July 1955. 
43. A.A. Townsend; Momentum and Energy Diffusion in the Turbulent Wake of a Cylinder; Proc. Royal Soc. Iondon, Vol. A 197, (1924), May 1949.

44. R.H. Kraichnan; Noise Transmission from Boundary Layer Pressure Fluctuations; J. Acoustic. Soc. Amer., Vol. 29, (65), Jan. 1957.

45. W.W. Willmarth; Wall Pressure Fluctuations in a Turbulent Boundary Layer; J. Acoustic. Soc.Amer., Vol. 28, (1048), Nov. 1956.

46. C.M. Ashley; Air Conditioning Noise Control; Noise Control, Vol. 1, (37), March 1955.

47. L.L. Beranek; Revised Criteria for Noise in Buildings; Noise Control, Vol. 3, (19), Jan. 1957.

48. R.F. Lambert; Acoustic Filtering in a Moving Medium; J. Acoustic. Soc. Amer., Vol. 28, (1054), Nov. 1956.

49. R.F. Lambert; Side Branch Insertion Loss in a Moving Medium, Vol. 28, (1059), Nov. 1956.

50. L.L. Beranek \& H.P. Sleeper; Design and Construction of Anechoic Sound Chambers; J. Acoustic. Soc. Amer., Vol. 18, (140), July 1946.

51. 4.J. King; The Reduction of Noise from Air Conditioning Systems; Engineering, Vol. 157, (501), June 1944.

52. J.H. Stiggleman; Large High Velocity Ventilating System; Heating, Piping and Air Conditioning; Vol. 26, (87), July 1954. 


\section{BOOKS}

53. J.W.S. Rayleigh; Theory of Sound Vol. I \& II; Dover Publications, New York; 2nd Ed., 1945.

54. R.D. Madison; Fan Engineering (Handbook); Buffalo Forge Co., Buffalo, N.Y.; 5th Ed., 1949.

55. F. Woods; Woods Practical Guide to Fan Engineering; Woods of Colchester Ltd., England; lst Ed., 1952.

56. P.M. Morse; Vibration and Sound; MeGraw - Hill Book Co. Inc.; 2nd Ed., 1948.

57. L.L. Beranek; Acoustics; McGraw - Hill Book Co. Inc., Ist ed., 1954 .

58. H.J. Reich, P.P. Ordung, H.L. Krauss, J.G. Skalnik; Microwave Theory and Techniques; D. Van Nostrand Co. Inc., Ist Ed., 1953.

59. A.H. Shapiro; The Dynamics and Thermodynamics of Compressible Fluid Flow, Vol. 2; The Fonald Press Co., New York; lst Ed., 1954.

60. H.F. Olson \& F. Massa; Aplied Acoustics; P. Blakestron's Son \& Co. Inc., 1934.

61. Heating, Ventilating \& Air Conditioning Guide; American Society of Heating \& Ventilating Engr.; New York, 1952.

62. A.P.G. Peterson \& I.L. Beranek; Handbook of Noise Measurement; General Radio Co., Cambridge, Mass., 1956.

63. E.E. Gross; Measurement of Vibration; General Radio Co., Cambridge, Mass., 1956. 


\section{STANDARDS}

64. American Standards Association

Z 24.1 - 1951 Acoustical Terminology.

Z 24.3 - 1944 Sound - Level Meters.

Z 24.7 - 1950 Test Code for Apparatus Noise Measurement.

Z24.10 - Octave - Band Filter Set for the Analysis of Noise and Other Sounds. 